\title{
Ellagitannins and Related Compounds from
}

\section{Penthorum chinense}

Manami Era, ${ }^{\dagger}, ‡$ Yosuke Matsuo,,${ }^{*} \dagger$ Yoshinori Saito,${ }^{\dagger}$ Koyo Nishida, ${ }^{\ddagger}$ Zhi-Hong Jiang, ${ }^{\S}$ and Takashi Tanaka, ${ }^{*} \dagger$

${ }^{\dagger}$ Department of Natural Product Chemistry, Graduate School of Biomedical Sciences, Nagasaki University, 1-14 Bunkyo-Machi, Nagasaki 852-8521, Japan

*Department of Pharmaceutics, Graduate School of Biomedical Sciences, Nagasaki University, 1-7-1 Sakamoto, Nagasaki 852-8501, Japan

${ }^{\S}$ Institute for Applied Research in Medicine and Health, Macau University of Science and Technology, Taipa, Macau 999078, People's Republic of China 
ABSTRACT: Four new ellagitannin metabolites, penthorumnins A-D (1-3, and 5), were isolated from the dried stem of Penthorum chinense. The structures were determined using spectroscopic and chemical analysis as well as using computations, that revealed the following: (1) the acyl group of penthorumnin A (1) has a unique cyclopentane carboxylic acid structure that is derived from a hexahydroxydiphenoyl (HHDP) group; (2) penthorumnin B (2) has a 2carboxymethyl-2,3-dihydro-3-oxo-1H-indene-1-carboxylic acid structure that originates from the acyl group of penthorumnin A; (3) penthorumnin C (3) is a glucoside of trihydroxyacetophenone with an acyl group that is oxidatively derived from the HHDP group. This acyl group is closely related to that of balanophotannin F (4), which has been previously isolated from Balanophora japonica and whose absolute configuration has been revised using the DFT method; and (4) penthorumnin $\mathrm{D} \quad(\mathbf{5})$ is defined as $2^{\prime}, 4^{\prime}, 6^{\prime}$-trihydroxyacetophenone $4^{\prime}-O-[4,6-(S)$ dehydrohexahydroxydiphenoyl]- $\beta$-glucoside. The variety of the acyl groups in these ellagitannins is indicative of the occurrence of a unique metabolism in this plant involving the HHDP ester. 
Penthorum chinense (Penthoraceae) is an herbaceous plant distributed in Eastern Asia. Its stems and leaves are traditionally used as functional tea and folk medicine for hepatic diseases. ${ }^{1,2}$ Recent studies concerning the hepatoprotective, hypoglycemic, and DNA damage-protective effects of this plant have demonstrated its biological activities, ${ }^{3-5}$ which have been associated with the antioxidant activity of polyphenols, ${ }^{6}$ especially of their major components, i.e., the hexahydroxydiphenoyl (HHDP) esters of flavonoid glycosides. ${ }^{4}$ During our chemical studies on polyphenols obtained from medicinal herbs and traditional food, we recently reported the presence of two rare ellagitannins having an (R)-HHDP group at C-4/C-6 of the glucose moiety, ${ }^{7}$ which indicated the occurrence of a unique ellagitannin metabolism in this plant because this HHDP group normally exhibited the $S$ configuration. ${ }^{8}$ Further chromatographic separation of the fractions obtained in our previous study resulted in the isolation of four new compounds along with two known phenolic compounds. This study deals with the isolation and structure determination of the new compounds. In addition, the structural similarities of one of the new compounds to balanophotannin $\mathrm{F}\left(\mathbf{4}^{\prime}\right)$, previously isolated from Balanophora japonica, ${ }^{9}$ prompted us to reinvestigate this ellagitannin using DFT calculations.

\section{RESULTS AND DISCUSSION}

The dried stems of $P$. chinense were extracted with $60 \%$ aq. acetone, and the extract was fractionated by Diaion HP20SS column chromatography to produce 12 fractions, as described in the previous study. ${ }^{7}$ Further chromatographic separation of the fractions using Sephadex LH-20, MCI-gel CHP20P, Chromatorex ODS, and Toyopearl HW40F afforded four new compounds (1$\mathbf{3}, \mathbf{5})$ and two known phenolic compounds that were identified as phyllanemblinin $\mathrm{F}(\mathbf{6})^{10}$ and chebulic acid (7) by comparison of their spectroscopic data with those of the authentic samples. 

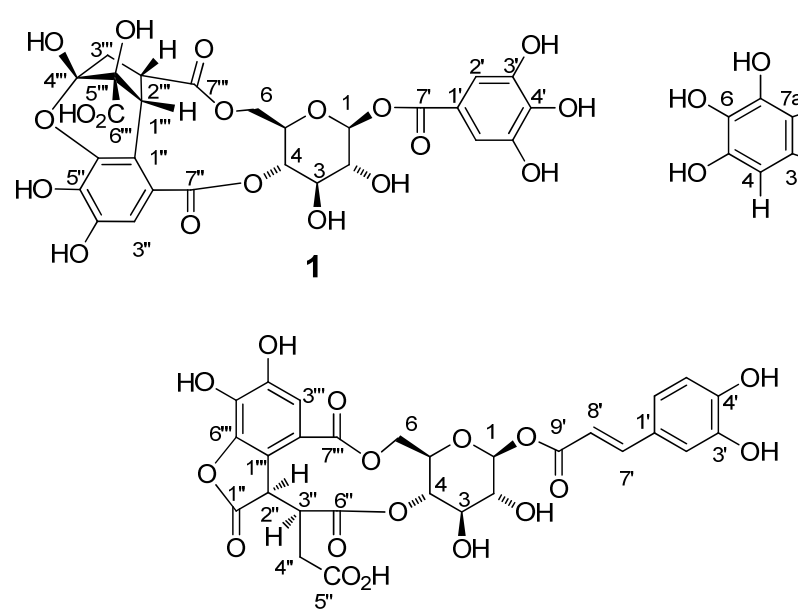

4

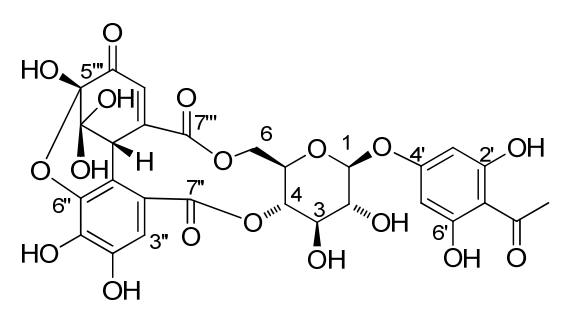

5

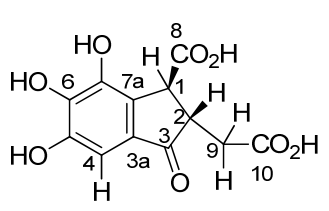

2

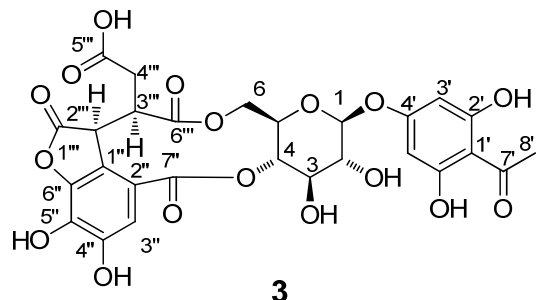

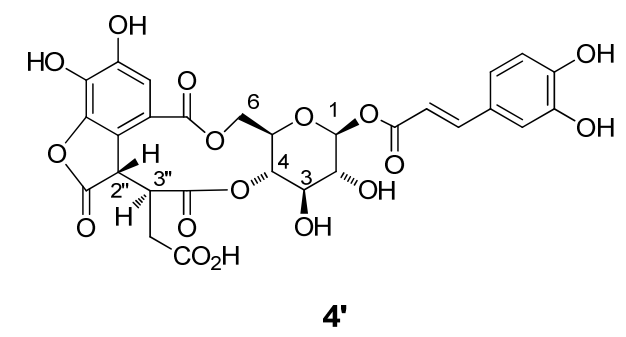

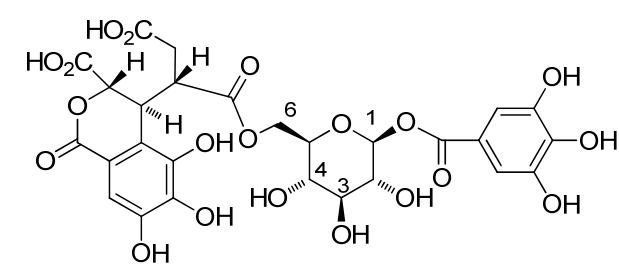

6

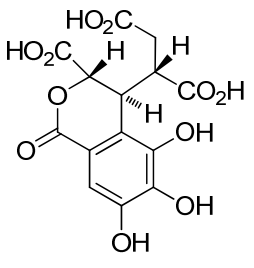

7

Figure 1. Structures of 1-7 and structure of balanophotannin F (4')

Penthorumnin A (1) was obtained as a brown amorphous powder, and its molecular formula was deduced as $\mathrm{C}_{27} \mathrm{H}_{24} \mathrm{O}_{19}$ from HRESIMS data, that exhibited the $[\mathrm{M}-\mathrm{H}]^{-}$peak at $m / z$ 651.0848 (calcd. for $\mathrm{C}_{27} \mathrm{H}_{23} \mathrm{O}_{19}, 651.0834$ ). The appearance of a prominent fragment ion attributable to $\left[\mathrm{M}-\mathrm{CO}_{2} \mathrm{H}\right]^{-}$at $m / z 607$ indicated the presence of a hydroxycarbonyl group. The ${ }^{1} \mathrm{H}$ NMR spectrum exhibited chemical shifts and coupling constants for the aliphatic proton signals of those of the 1-O-galloyl-4,6-(S)-HHDP- $\beta$-D-glucose (strictinin) moiety, ${ }^{7}$ supporting the assignment of 1 as a 1-O-galloyl-4,6-acylglucose (Table 1). The glucose moiety was assumed as 
belonging to the D-series based on the D-glucose core of ellagitannins obtained from the same plant source. ${ }^{7}$

Table 1. ${ }^{1} \mathrm{H}(500 \mathrm{MHz})$ and ${ }^{13} \mathrm{C}(125 \mathrm{MHz}) \mathrm{NMR}$ data for Penthorumnins A (1), C (3) and D (5) ( $\delta$ in ppm, $J$ in $\mathrm{Hz}$, Measured in methanol- $d_{4}$.)

\begin{tabular}{|c|c|c|c|c|c|c|c|c|c|}
\hline \multirow{2}{*}{\multicolumn{2}{|c|}{ Position }} & \multicolumn{3}{|c|}{ 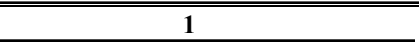 } & \multicolumn{2}{|l|}{3} & \multicolumn{3}{|c|}{5} \\
\hline & & \multicolumn{2}{|l|}{${ }^{1} \mathrm{H}$} & \multirow{2}{*}{$\frac{{ }^{13} \mathrm{C}}{95.6}$} & \multirow{2}{*}{$\frac{{ }^{1} \mathrm{H}}{5.17 \mathrm{~d}(7.7)}$} & \multirow{2}{*}{$\frac{{ }^{13} \mathrm{C}}{100.9}$} & \multicolumn{2}{|c|}{${ }^{1} \mathrm{H}$} & \multirow{2}{*}{$\frac{{ }^{13} \mathrm{C}}{100.1}$} \\
\hline glucose & 1 & 5.69 & $\mathrm{~d}(8.0)$ & & & & 5.22 & $\mathrm{~d}(7.6)$ & \\
\hline & 2 & 3.66 & dd $(8.0,9.2)$ & 74.1 & \multirow{2}{*}{$3.65 \mathrm{~m}$} & 74.5 & 3.63 & dd $(7.6,9.3)$ & 74.1 \\
\hline & 3 & 3.77 & $\mathrm{t}(9.3)$ & 75.9 & & 75.9 & 3.87 & $\mathrm{t}(9.3)$ & 74.4 \\
\hline & 4 & 5.00 & $\mathrm{t}(9.3)$ & 75.4 & $5.06 \mathrm{~m}$ & 76.2 & 5.20 & $\mathrm{t}(9.3)$ & 75.2 \\
\hline & 5 & 3.98 & ddd $(6.2,6.4,9.3)$ & 71.2 & $4.23 \mathrm{dt}(2.7,9.8)$ & 70.4 & 4.02 & $\mathrm{dt}(4.9,10.7)$ & 68.7 \\
\hline & 6 & 4.64 & $\mathrm{dd}(6.1,12.1)$ & 66.5 & $4.04 \mathrm{dd}(2.7,11.0)$ & 64.1 & 3.88 & $\mathrm{t}(10.7)$ & 66.1 \\
\hline & & 3.72 & $\mathrm{dd}(6.4,12.1)$ & & $5.12 \mathrm{dd}(9.8,11.0)$ & & 4.97 & $\mathrm{dd}(4.9,10.7)$ & \\
\hline \multirow[t]{5}{*}{ galloyl } & $1^{\prime}$ & & & 120.4 & & & & & \\
\hline & $2^{\prime}, 6^{\prime}$ & 7.13 & $\mathrm{~s}$ & 110.5 & & & & & \\
\hline & $3^{\prime}, 5^{\prime}$ & & & 146.5 & & & & & \\
\hline & $4^{\prime}$ & & & 140.4 & & & & & \\
\hline & $7^{\prime}$ & & & 166.7 & & & & & \\
\hline \multirow[t]{6}{*}{ acetophenone } & $1^{\prime}$ & & & & & 106.7 & & & 106.8 \\
\hline & $2^{\prime}, 6^{\prime}$ & & & & & 164.9 & & & 165.0 \\
\hline & $3^{\prime}, 5^{\prime}$ & & & & $6.18 \mathrm{~d}(1.7)$ & 95.9 & 6.10 & $\mathrm{~s}$ & 96.1 \\
\hline & $4^{\prime}$ & & & & & 164.3 & & & 164.3 \\
\hline & $\mathrm{CO}$ & & & & & 204.4 & & & 204.4 \\
\hline & $\mathrm{CH}_{3}$ & & & & $2.62 \mathrm{~s}$ & 32.9 & 2.59 & $\mathrm{~s}$ & 32.9 \\
\hline \multirow[t]{16}{*}{ 4,6-acyl } & $1 "$ & & & 115.4 & & 115.6 & & & 113.0 \\
\hline & $2^{\prime \prime}$ & & & 123.9 & & 120.0 & & & 124.5 \\
\hline & $3 "$ & 6.59 & $\mathrm{~s}$ & 108.4 & $7.18 \mathrm{~s}$ & 114.3 & 6.70 & $\mathrm{~s}$ & 108.2 \\
\hline & $4 "$ & & & 146.1 & & 147.5 & & & 146.2 \\
\hline & $5^{\prime \prime}$ & & & 136.2 & & 134.9 & & & 135.4 \\
\hline & $6^{\prime \prime}$ & & & 142.6 & & 142.6 & & & 142.4 \\
\hline & $7 "$ & & & 169.4 & & 165.6 & & & 168.4 \\
\hline & $1 " '$ & 4.33 & br d (5.9) & 46.4 & & 175.6 & 4.70 & $\mathrm{~s}$ & 43.0 \\
\hline & $2 " '$ & 3.66 & ddd $(5.9,7.5,10.5)$ & 48.3 & $4.64 \mathrm{~d}(3.2)$ & 46.9 & & & 151.2 \\
\hline & $3^{\prime \prime \prime}$ & 2.28 & ddd $(1.1,10.5,14.6)$ & 37.8 & $3.44 \operatorname{ddd}(2.8,3.2,11.9)$ & 43.6 & 6.75 & $\mathrm{~s}$ & 130.8 \\
\hline & & 2.60 & $\mathrm{dd}(7.5,14.6)$ & & & & & & \\
\hline & $4 " '$ & & & 108.2 & $1.93 \mathrm{dd}(2.8,17.2)$ & 30.4 & & & 192.1 \\
\hline & & & & & $2.14 \mathrm{dd}(11.9,17.2)$ & & & & \\
\hline & $5^{\prime \prime \prime}$ & & & 80.1 & & 172.2 & & & 96.6 \\
\hline & $6^{\prime \prime \prime}$ & & & 172.7 & & 172.1 & & & 91.7 \\
\hline & $7 " '$ & & & 173.7 & & & & & 164.5 \\
\hline
\end{tabular}

In the same spectrum, the presence of an aromatic proton singlet at $\delta 6.59$ and six aromatic carbon signals related to those of the trihydroxybenzoyl group suggested the presence of a pyrogallol-type aromatic ring in the 4,6-acyl group (Table 1). HMBC correlations of the aromatic singlet (H-3") and glucose $\mathrm{H}-4(\delta 5.00)$ with an ester carbonyl carbon resonating at $\delta$ 173.7 (C-7") confirmed that the aromatic carboxy group was attached to the 4-hydroxy group of 
glucose (Figure 1). The remaining aliphatic proton signals at $\delta 4.33$ (br. d, $J=5.9 \mathrm{~Hz}$ ), $\delta 3.66$ $(\mathrm{ddd}, J=5.9,7.5,10.5 \mathrm{~Hz}), \delta 2.28(\mathrm{ddd}, J=1.1,10.5,14.6 \mathrm{~Hz})$, and $\delta 2.60(\mathrm{dd}, J=7.5,14.6 \mathrm{~Hz})$ indicated the presence of a partial structure of $\mathrm{CH}-\mathrm{CH}-\mathrm{CH}_{2}(\mathrm{C}-1$ "', $\mathrm{C}-2$ "', and $\mathrm{C}-3$ "', respectively), and this was confirmed by a ${ }^{1} \mathrm{H}-{ }^{1} \mathrm{H}$ COSY spectrum. In addition, the carbon signals at $\delta 80.1$ and 108.2 were attributable to an oxygen-bearing tertiary carbon (C-5"') and an acetal carbon (C-4"'), respectively. The carboxy carbonyl deduced from the abovementioned MS fragmentation resonated at $\delta 172.7$ (C-6"').

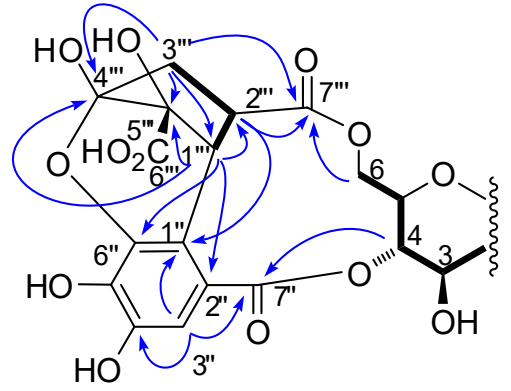

selected HMBC of 1

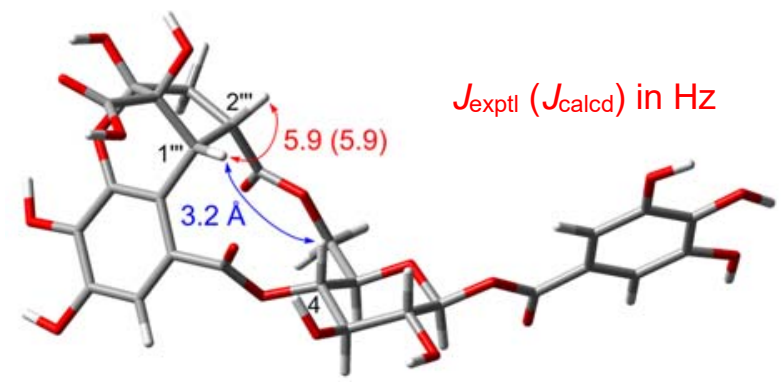

lowest-energy conformer of 1 and NOE (blue arrow)

Figure 2. Partial structure of 1 with selected HMBC correlations, a key NOE, and a coupling constant, and the alternative structure 1'.

In the HMBC spectrum, correlations of the methine H-1"' to C-2", C-6", C-4"', and C-5"', of $\mathrm{H}-2$ "' to C-1" and C-7"', and of H-3"' to C-1"', C-4"', C-5"', and C-7"' indicated the presence of a five-membered ring (Figure 2). The acetal carbon C-4"' resonated at relatively lower field $(\delta$ 
108.2) compared to $\mathrm{C}-1$ of glucose ( $\delta 95.6)$, which suggested the formation of an ether ring at this position with the C-6" phenolic group that would be consistent with the index of hydrogen deficiency (16). Although it is apparent that the hemiacetal ring formation with the phenolic group at C-6" occurs from the same side of the phenyl group at C-1"', the reverse assignment as hemiacetal C-4"' and oxygenated tertiary C-5"' depicted in the partial structure of 1 ' cannot be ruled out. Since the configuration at C-5"' was also ambiguous, DFT calculations of the possible 16 isomers were performed (Figures S1-S6, supporting information). It was found that only four structures $\left[\left(1 " ' R, 2\right.\right.$ "'S,4"'R,5"'R)-1A $\left(J_{\text {calcd }}=5.9 \mathrm{~Hz}\right) ;\left(1 " ' R, 2 " ' S, 4 " ' R, 5{ }^{\prime \prime} S\right)-\mathbf{1 B}\left(J_{\text {calcd }}=6.3 \mathrm{~Hz}\right)$; $(1 " ' S, 2 " ' R, 4 " ' S, 5 " ' S)-1 \mathrm{C}\left(J_{\text {calcd }}=4.9 \mathrm{~Hz}\right)$; and $\left.(1 " ' S, 2 " ' R, 4 " ' S, 5 " ' R)-1 \mathrm{D}\left(J_{\text {calcd }}=5.1 \mathrm{~Hz}\right)\right]$ having a six-membered hemiacetal ring matched well the experimental ${ }^{1} \mathrm{H}$ NMR coupling constant of 5.9 $\mathrm{Hz}$ between $\mathrm{H}-1$ "' and H-2"'. Comparison of the experimental and calculated ${ }^{13} \mathrm{C}$ NMR chemical shifts with those obtained experimentally indicated that $(1 " ' R, 2 " ' S, 4 " ' R, 5 " ' R)-\mathbf{1 A}\left(R^{2}=0.9980\right)$ and $(1 " ' R, 2 " ' S, 4 " ' R, 5 " ' S)$-1B $\left(R^{2}=0.9977\right)$ were the most reasonable candidates. Furthermore, the calculated ${ }^{1} \mathrm{H}$ NMR chemical shifts of $(1 " ' R, 2 " ' S, 4 " ' R, 5 " ' R)-\mathbf{1 A}\left(R^{2}=0.9920\right)$ were more consistent with the experimental data than those of other possible structures such as (1"'R,2"'S,4"'R,5"'S)-1B $\left(R^{2}=0.9854\right)$. Meanwhile, comparison of the calculated ECD spectra of

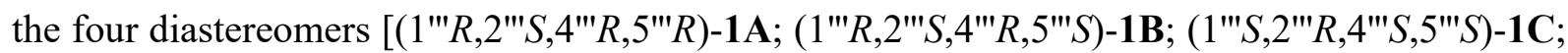
and $(1 " ' S, 2$ "'R,4"'S,5"'R)-1D] with the experimental ECD spectrum of 1 also supported the configuration of the acyl moiety (Figure 3). On the basis of these results, penthorumnin A (1) was defined as (1"'R,2"'S,4"'R,5"'R)-1A (Figure 2). 


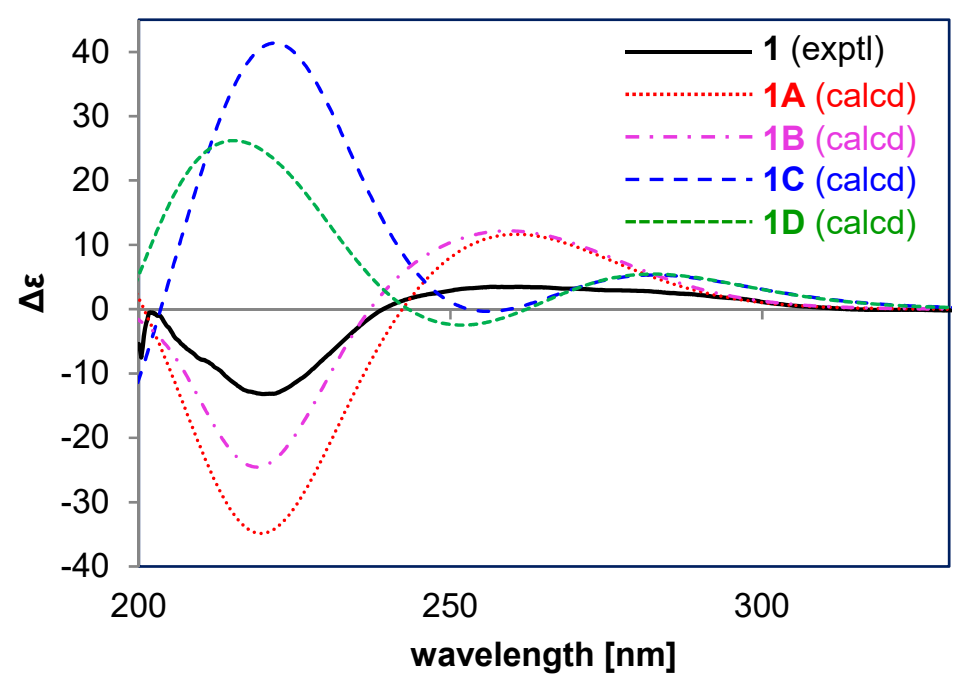

Figure 3. Experimental and calculated ECD spectra of $\mathbf{1}\left(\mathbf{1 A}: 1{ }^{\prime \prime} R, 2\right.$ "'S,4"'R,5"'R; 1B: 1 "'R,2"'S,4"'R,5"'S; 1C: 1 "'S,2"'R,4"'S,5"'S; 1D: 1 "'S,2"'R,4"'S,5"'R). The experimental ECD spectrum was measured in $\mathrm{MeOH}$. The calculations for the ECD spectra were performed at the TD-CAM-B3LYP/6-31G(d,p) level in MeOH (PCM). The calculated spectra were red-shifted by $10 \mathrm{~nm}$ with respect to the experimental spectrum.

Penthorumnin B (2) was isolated as a brown amorphous powder, and its molecular formula was determined to be $\mathrm{C}_{12} \mathrm{H}_{10} \mathrm{O}_{8}$ by HRFABMS data on the basis of the $[\mathrm{M}+\mathrm{H}]^{+}$peak at $m / z 283.0458$ [calcd. for $\mathrm{C}_{12} \mathrm{H}_{11} \mathrm{O}_{8}, 283.0458$ ]. The ESIMS (negative mode) showed two prominent fragment ions at $m / z 237$ and 193 corresponding to $\left[\mathrm{M}-\mathrm{H}-\mathrm{CO}_{2}\right]^{-}$and $\left[\mathrm{M}-\mathrm{H}-2 \mathrm{CO}_{2}\right]^{-}$, respectively, suggesting the presence of two hydroxycarbonyl groups. The ${ }^{1} \mathrm{H}$ NMR and ${ }^{1} \mathrm{H}-{ }^{1} \mathrm{H}$ COSY spectra exhibited signals attributable to the partial structure $\mathrm{CH}-\mathrm{CH}-\mathrm{CH}_{2}[\delta 3.96(\mathrm{~d}, J=$ $4.1 \mathrm{~Hz}, \mathrm{H}-1), 3.05$ (dt, $J=4.3,7.3 \mathrm{~Hz}, \mathrm{H}-2), 2.86(\mathrm{dd}, J=4.3,17.2 \mathrm{~Hz}, \mathrm{H}-9 \mathrm{a})$, and 2.75 (dd, $J=$ 7.3, $17.2 \mathrm{~Hz}, \mathrm{H}-9 \mathrm{~b})]$, together with an aromatic proton $(\delta 6.74, \mathrm{H}-4)$. The ${ }^{13} \mathrm{C}$ NMR spectrum exhibited 10 carbon signals that can be assigned to a trioxygenated aromatic ring [148.5 (C-6), 143.5 (C-5), 142.6 (C-7), 132.3 (C-3a), 128.2 (C-7a), 101.7 (C-4)], a ketocarbonyl ( $\delta$ 206.1, C-3), two hydroxycarbonyl groups ( $\delta 177.6, \mathrm{C}-8 ; 175.0, \mathrm{C}-10)$, and the aforementioned partial structure 
( $\delta$ 49.5, C-1; 49.8, C-2; 35.1, C-9), respectively. In the HMBC spectrum, the aromatic H-4 correlated with the ketocarbonyl C-3 and the aromatic carbons C-3a, C-5, C-6, and C-7 (Figure 4). The aromatic C-3a, C-6, C-7, and C-7a showed cross peaks with the methine H-1 ( $\delta 3.96)$. The locations of the two hydroxycarbonyl groups at C-9 and C-10 were confirmed via the HMBC correlations with H-1, H-2, and H-9. Furthermore, the correlations of C-3 with H-4 and H-9 allowed establishment of the 2D structure of 2. The trans orientation of H-1 and H-2 was deduced from the NOE correlation between H-1 and H-9 (Figure 4), and the $(1 R, 2 R)$ absolute configuration was defined by comparison of the experimental and calculated ECD spectra (Figure 5). In addition, calculation of the specific rotation of $(1 R, 2 R)-2$ performed at B3LYP/6$311+\mathrm{G}(2 \mathrm{~d}, \mathrm{p})$ level in $\mathrm{MeOH}(\mathrm{PCM})$ afforded $[\alpha]_{\mathrm{D}}-129.9$, the negative sign being consistent with the experimental value (-26.8) (Tables S67, 68, supporting information). From these results, the structure of penthorumnin B (2), was established as depicted in Figure 1. The structure suggested the biosynthetic relationship between $\mathbf{2}$ and the 4,6-acyl group of $\mathbf{1}$, and a putative biosynthesis is proposed in Scheme 1.
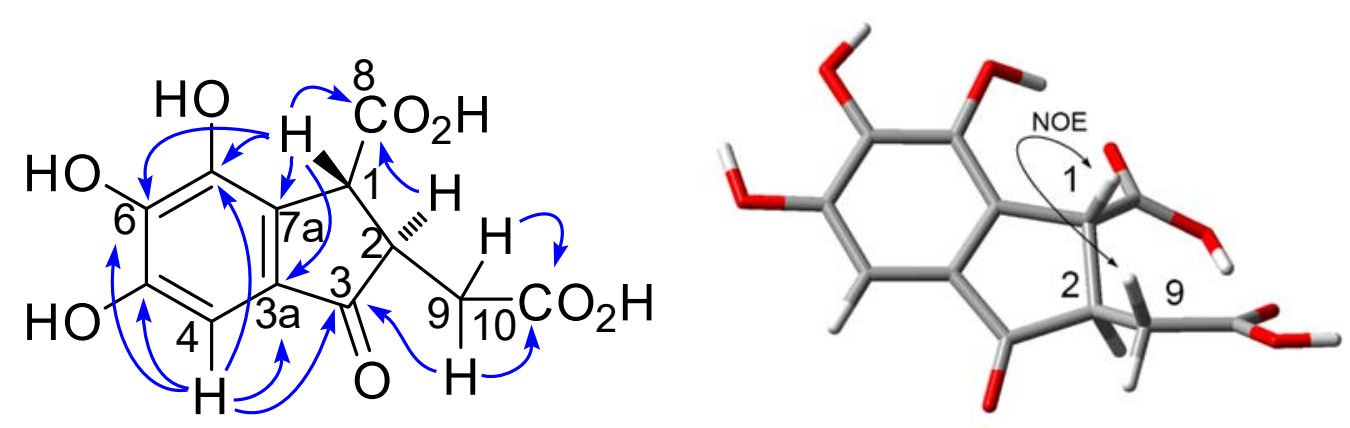

Figure 4. HMBC correlations of $\mathbf{2}$ and the most stable conformer of $(1 R, 2 R)-\mathbf{2}$. 


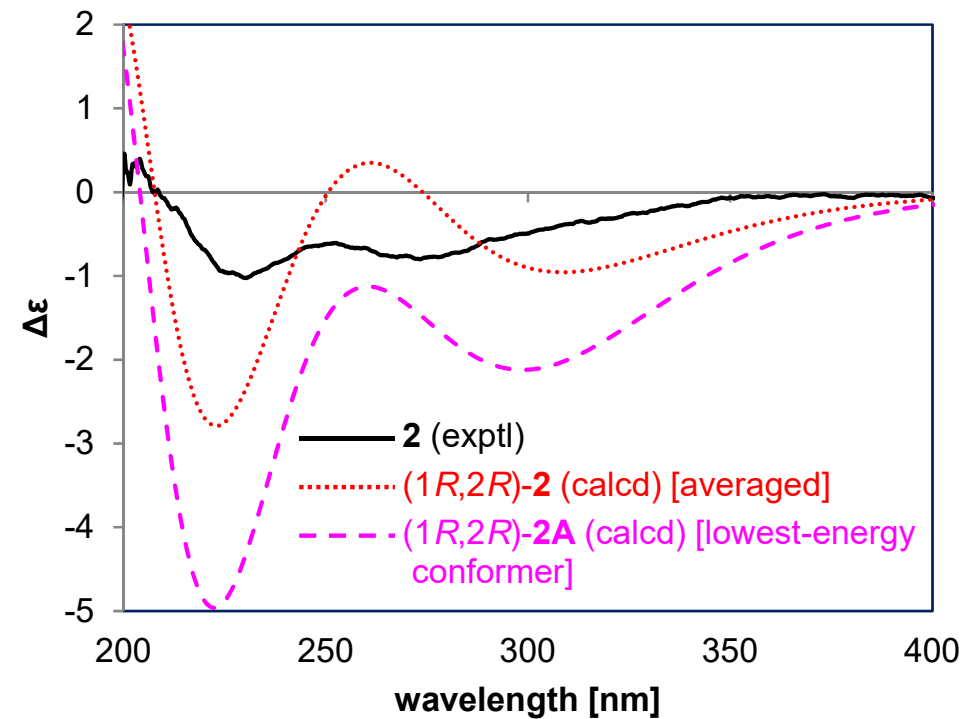

Figure 5. Experimental and calculated ECD spectra of 2. Calculation of ECD spectra was performed at the TD-BH\&HLYP/6-311+G(2d,p) level in MeOH (PCM).
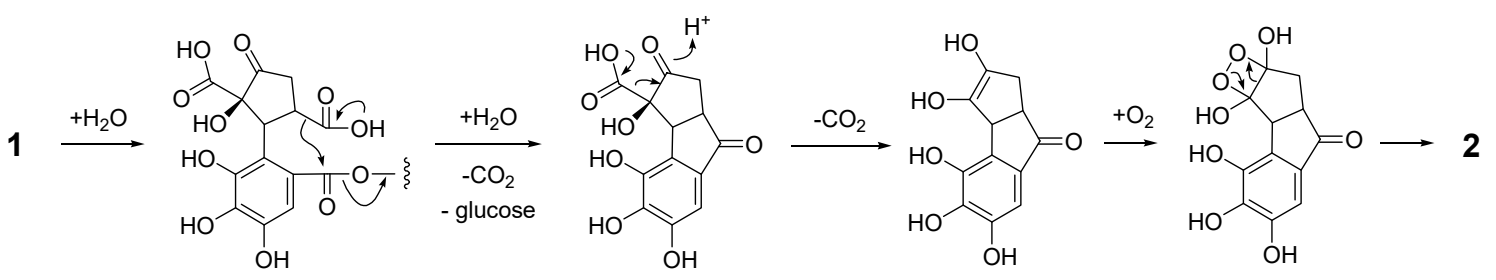

Scheme 1. Putative biosynthetic conversion of 1 to 2 .

The ${ }^{1} \mathrm{H}$ and ${ }^{13} \mathrm{C}$ NMR spectra of penthorumnin $\mathrm{C}(3)$ showed signals assignable to a trihydroxyacetophenone 4-O-glucopyranosyl moiety (Table 1 ) that were similar to those of 2',4',6'-trihydroxyacetophenone 4 '- $O-[4,6-(S)$-HHDP]- $\beta$-glucoside, isolated in the previous study. ${ }^{7}$ The large difference between the chemical shifts of the C-6 methylene protons of glucose ( $\delta 4.04$ and 5.12) indicated the presence of an acyl bridge across the 4,6-hydroxy groups of glucose. ${ }^{8}$ From the ${ }^{13} \mathrm{C}$ NMR spectrum, it can be concluded that the 4,6-acyl group has a trihydroxybenzoyl moiety (C-1"-C-7"), three carboxy groups (C-1"', C-5"', and C-6"'), two 
mutually coupled methines (C-2"' and C-3"'), and a methylene (C-4"'). On the basis of the HMBC and ${ }^{1} \mathrm{H}-{ }^{1} \mathrm{H}$ COSY correlations of these carbons, the 2D structure depicted in Figure 6 was constructed. The molecular formula $\mathrm{C}_{27} \mathrm{H}_{24} \mathrm{O}_{17}$ was established by HRFABMS, which showed the $[\mathrm{M}+\mathrm{H}]^{+}$ion at $m / z 621.1091$ (calcd for $\mathrm{C}_{27} \mathrm{H}_{25} \mathrm{O}_{17}, 621.1092$ ), and the hydrogen deficiency index was in accord with lactone-ring formation between the C-6" phenolic group and the C-1"' carboxy group. This was strongly supported by a characteristic IR absorption at $1804 \mathrm{~cm}^{-1}$. To confirm the stereostructure, the possible stereoisomers of $\mathbf{3}$, i.e., $(2 " ' S, 3 " \prime R)-\mathbf{3 A},\left(2 " ' R, 3^{\prime \prime \prime} R\right)-\mathbf{3 B}$, (2"'S,3"'S)-3C, and (2"'R,3"'S)-3D, were subjected to DFT calculation, and the experimental and calculated $J_{2 " '-3{ }^{\prime \prime \prime}}$ values were compared (Figures S7-S10, supporting information). The calculated

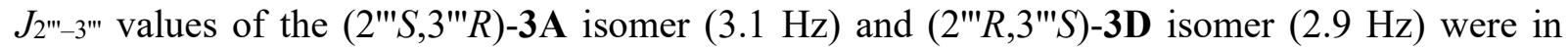
agreement with the experimental value of $\mathbf{3}(3.2 \mathrm{~Hz})$, whereas the $(2 " ' R, 3 " ' R)-3 \mathbf{B}$ and $(2 " ' S, 3$ "'S)3C isomers can be ruled out on the basis of their calculated values $(10.4$ and $11.6 \mathrm{~Hz}$, respectively) (Supporting information, Figure S8). Finally, the absolute configuration of $\mathbf{3}$ was confirmed as $(2 " ' S, 3$ "'R) by comparison between the experimental and calculated ECD spectra of the $(2 " ' S, 3 " ' R)-\mathbf{3 A}$ and $(2 " ' R, 3$ "'S)-3D isomers (Figure 7).

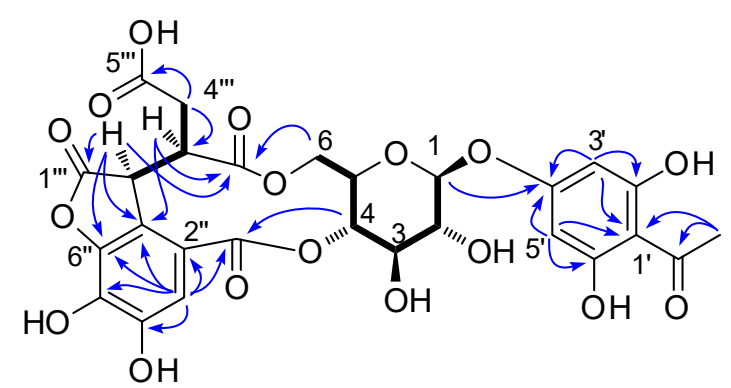

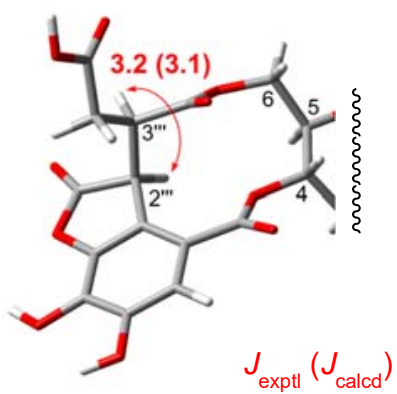

$(2$ "'S,3"'R)

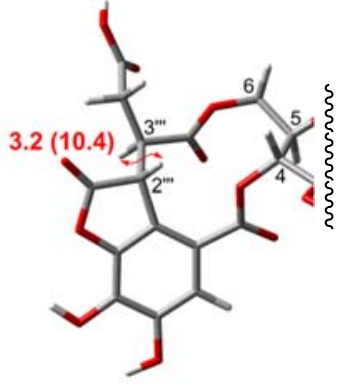

$(2 " ' R, 3 " ' R)$

Figure 6. HMBC and ${ }^{1} \mathrm{H}-{ }^{1} \mathrm{H}$ COSY correlations of $\mathbf{3}$ (blue arrows and black bold lines, respectively), and two possible relative structures $(2 " ' S, 3 " ' R)-\mathbf{3 A}$ and $(2 " ' R, 3 " ' R)-\mathbf{3 B}$. The values are experimental coupling constants and calculated coupling constants (in parentheses). 


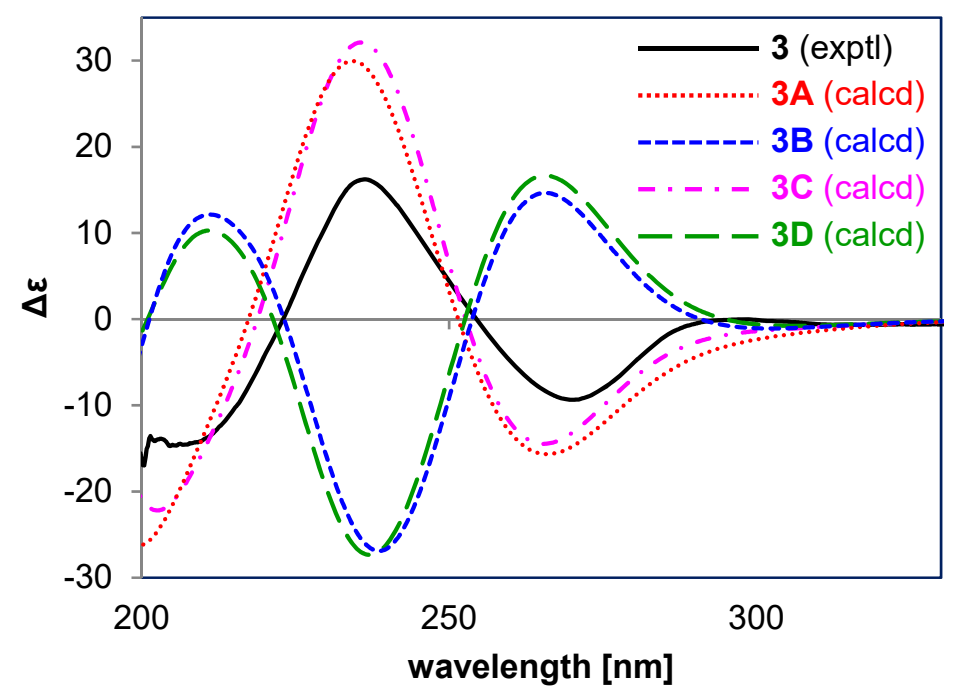

Figure 7. Experimental and calculated ECD spectra of $\mathbf{3}$ (3A: 2 "'S,3"' $R$; 3B: 2 "'R,3"' $R$; $3 \mathbf{C}$ : $2 " ' S, 3$ "'S; 3D: 2"'R,3"'S). The experimental ECD spectrum was measured in MeOH. The calculations for the ECD spectra were performed at the TD-CAM-B3LYP/6-31G(d,p) level in $\mathrm{MeOH}(\mathrm{PCM})$. The calculated spectra were red-shifted by $15 \mathrm{~nm}$.

Interestingly, the 2D structure of the acyl group of $\mathbf{3}$, as well as the coupling constants of H-2"', H-3"' and H-4"', were virtually the same as those of balanophotannin F (4'). However, 4' was reported to have a $(2 " S, 3 " S)$ configuration (Figure 1). ${ }^{9}$ Therefore, the structure of balanophotnnin F was reinvestigated by a similar computational method to define it as 4 (Figures S11-S14, supporting information).

Penthorumnin D (5) was isolated as a yellow amorphous powder, and the $[\mathrm{M}+\mathrm{H}]^{+}$ion at $m / z 649.1014$ in the HRFABMS data indicated that the molecular formula was $\mathrm{C}_{28} \mathrm{H}_{24} \mathrm{O}_{18}$ (calcd. for $\mathrm{C}_{28} \mathrm{H}_{25} \mathrm{O}_{18}, 649.1041$ ). The ${ }^{1} \mathrm{H}$ and ${ }^{13} \mathrm{C}$ NMR data (Table 1) were similar to those of $\mathbf{3}$, showing the signals arising from a 4,6-acylated glucopyranosyl trihydroxyacetophenone moiety. The remaining signals suggested the presence of a DHHDP group, which was confirmed by the preparation of 2',4',6'-trihydroxyacetophenone 4'-O-[4,6-(S)-HHDP]- $\beta$-glucoside $(\mathbf{5 a})$, isolated from the same plant source in the previous study, ${ }^{7}$ by reduction of 5 with dithiothreitol. 


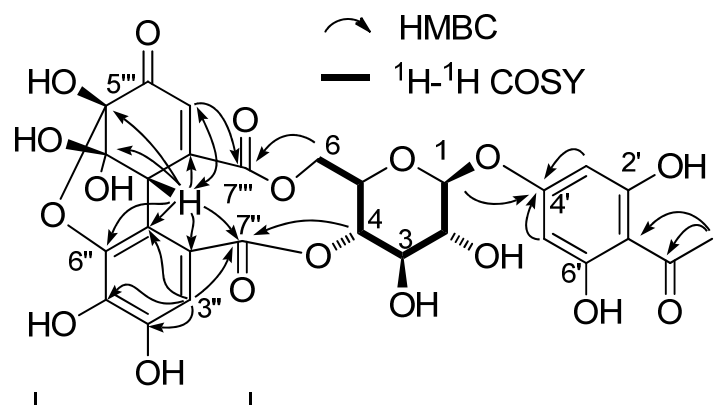

(S)-DHHDP
5<smiles>CC(=O)c1c(O)cc(OC2OC(COC(=O)c3cc(O)c(O)c(O)c3-c3cc(O)c(O)c(O)c3C(=O)O)[C@H](O)[C@H](O)[C@H]2O)cc1O</smiles>

$5 a$

Figure 8. Selected HMBC and ${ }^{1} \mathrm{H}-{ }^{1} \mathrm{H}$ COSY correlations of 5 and structure of the reduction product 5a.

In the HMBC spectrum of 5, the ester carbonyl C-7"' was correlated with the DHHDP olefinic H-3"' and H-6 of the glucosyl unit. The other ester carbonyl, C-7", showed cross peaks with the aromatic $\mathrm{H}-3$ " and $\mathrm{H}-4$ of the glucosyl moiety. These correlations determined the orientation of the DHHDP group on the glucopyranose ring. By analogy with the configuration of the $(S)$-HHDP group obtained from the abovementioned reduction with dithiothreitol, the same $(S)$ absolute configuration was assigned to the methine C-1"' of DHHDP in $5 .{ }^{11}$ This was confirmed by the corresponding ECD spectrum, which showed positive and negative Cotton effects at 236 and $270 \mathrm{~nm}$, respectively. From these results, the structure of penthorumnin D (5) was defined as 2',4',6'-trihydroxyacetophenone 4'-O-[4,6-(S)-DHHDP]- $\beta$-glucoside.

HPLC analysis confirmed the presence of the new compounds $\mathbf{1}-\mathbf{3}$, and $\mathbf{5}$ in the extract of the dried stem used in this study; however, $\mathbf{1}-\mathbf{3}$ were not detected in the fresh stem and the concentration of $\mathbf{5}$ in the fresh stem was much higher than dried stem (Figure S29, supporting information). This indicated that $\mathbf{5}$ was decomposed and $\mathbf{1}-\mathbf{3}$ are generated during the drying process. A plausible mechanism for the production of the acyl groups, including the chebuloyl group of 6, is depicted in Scheme 2. Since both $(S)$ - and (R)-HHDP groups exist in this plant, ${ }^{7}$ 
the different configuration of the two methine carbons in the acyl groups and their respective reaction metabolisms may reflect the atropisomerism of the original HHDP groups. Interestingly, the oxidation state of the acyl groups of $\mathbf{1}$ and $\mathbf{6}$ is the same as that of the HHDP group despite the presence of the hydroxycarbonyl groups, whereas the 4,6-acyl groups of $\mathbf{3}$ and $\mathbf{4}$ and the DHHDP group of $\mathbf{5}$ exhibited higher oxidation states than the HHDP group. Although the HHDP group was shown to be biosynthesized from the galloyl group, ${ }^{12}$ the detailed mechanisms have not been clarified yet. The biosynthetic relationship between DHHDP and HHDP is also ambiguous, and all reactions depicted in Scheme 2 are only tentatively proposed from the structures. However, non-enzymatic production of HHDP from DHHDP were previously reported; ${ }^{11 \mathrm{c}, 18}$ thus, the acyl groups of $\mathbf{1}, \mathbf{3}, \mathbf{4}$, and $\mathbf{6}$ are possibly produced from DHHDP. Further chemical and biological studies on the HHDP esters are thus needed to gain a better understanding of ellagitannin biosynthesis.

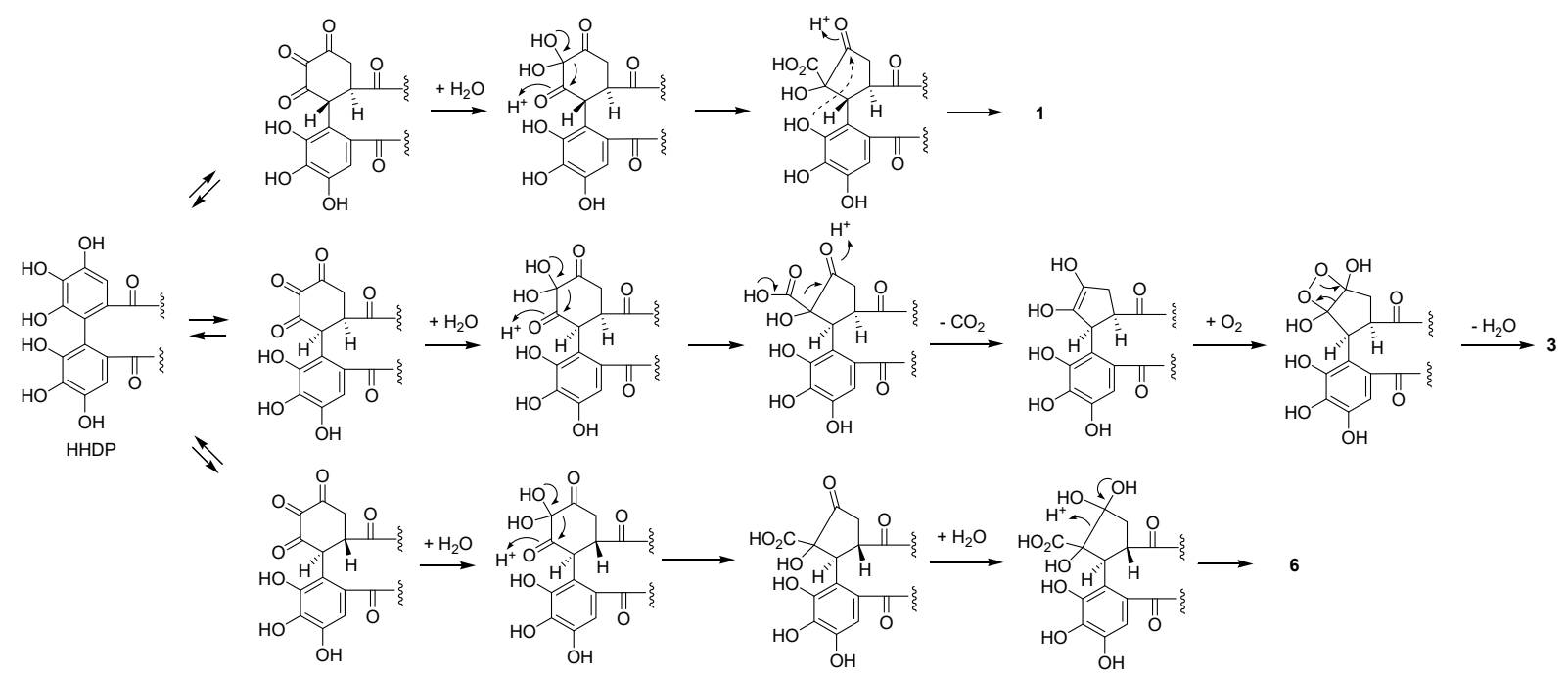

Scheme 2. Possible biosynthesis of $\mathbf{1 , 3}$, and $\mathbf{6}$ from the HHDP group. 


\section{EXPERIMENTAL SECTION}

General Experimental Procedures. Ultraviolet (UV) spectra were obtained on a JASCO V-560 UV/VIS spectrophotometer (JASCO, Tokyo, Japan). Optical rotations were measured with a JASCO DIP-370 digital polarimeter. The ECD spectra were measured with a JASCO J-725N spectrophotometer. ${ }^{1} \mathrm{H}$ and ${ }^{13} \mathrm{C}$ NMR spectra were recorded on a Varian Unity Plus 500 spectrometer (Agilent Technologies, Santa Clara, CA, USA) operating at 500 and 125 $\mathrm{MHz}$ for the ${ }^{1} \mathrm{H}$ and ${ }^{13} \mathrm{C}$ nuclei, respectively. NMR spectra were also recorded on a JEOL JNMAL 400 spectrometer (JEOL Ltd, Tokyo, Japan) operating at 400 and $100 \mathrm{MHz}$ for the ${ }^{1} \mathrm{H}$ and

${ }^{13} \mathrm{C}$ nuclei, respectively. ESIMS data were obtained using a JEOL JMS-T100TD spectrometer. FAB-MS were recorded on a JMS700N spectrometer (JEOL Ltd) using $m$-nitrobenzyl alcohol or glycerol as the matrix. Column chromatography was performed using Sephadex LH-20 (25-100 mm, GE Healthcare UK Ltd., Little Chalfont, UK), MCI-gel CHP20P (75-150 mm, Mitsubishi Chemical Co., Tokyo, Japan), Diaion HP20SS (Mitsubishi Chemical Co.), Toyopearl Butyl-650C (Tosoh Bioscience Japan, Tokyo, Japan), Cosmosil 75C18OPN (Nacalai Tesque Inc., Kyoto, Japan), and Chromatorex ODS (Fuji Silysia Chemical Ltd., Kasugai, Japan) columns. TLC was performed on precoated Kieselgel $60 \mathrm{~F}_{254}$ plates $(0.2 \mathrm{~mm}$ thickness, Merck, Darmstadt, Germany), using toluene-ethyl formate-formic acid (1:7:1, v/v) and $\mathrm{CHCl}_{3}-\mathrm{MeOH}-\mathrm{H}_{2} \mathrm{O}$ $(7: 3: 0.5, \mathrm{v} / \mathrm{v})$ mixtures as the eluents. The spots were detected using ultraviolet illumination and by spraying with $2 \%$ ethanolic $\mathrm{FeCl}_{3}$ solution (for phenolic compounds), $5 \%$ anisaldehyde in ethanolic $5 \% \mathrm{H}_{2} \mathrm{SO}_{4}$ solution (for proanthocyanidins), or $5 \% \mathrm{H}_{2} \mathrm{SO}_{4}$ solution followed by heating. Analytical HPLC was performed on a Cosmosil 5C18-ARII (Nacalai Tesque Inc., Kyoto, Japan) column $(250 \times 4.6 \mathrm{~mm}$, i.d. $)$ with a gradient elution of 4-30\% (39 $\mathrm{min})$ and 30 
$75 \%$ (15 min) $\mathrm{CH}_{3} \mathrm{CN}$ in $50 \mathrm{mM} \mathrm{H} \mathrm{PO}_{4}$ at $35{ }^{\circ} \mathrm{C}$ (flow rate, $0.8 \mathrm{~mL} / \mathrm{min}$; detection, JASCO photodiode array detector MD-2018 plus).

Plant Material. Dried stem material of P. chinense produced in December 2010 in Sichuan, China, was purchased at a local market by Dr. Zhi-Hong Jiang. The voucher specimen was deposited in the Graduate School of Biomedical Sciences, Nagasaki University.

Extraction and Separation. Dried stem material $(1.0 \mathrm{~kg})$ of $P$. chinense was crushed using a Waring blender and extracted with $60 \%$ acetone $(3 \times 3 \mathrm{~L})$. After evaporation of the solvent using a rotary evaporator, the resulting precipitates (Fr. 13, $42.3 \mathrm{~g}$ ) were collected by filtration. The filtrate was fractionated by Diaion HP20SS column chromatography $(7 \mathrm{~cm}$ i.d. $\times$ $34 \mathrm{~cm})$ with $\mathrm{H}_{2} \mathrm{O}$ containing increasing proportions of $\mathrm{MeOH}(0-100 \%, 10 \%$ stepwise elution, each $1 \mathrm{~L}$ ) and finally with $60 \%$ acetone $(3 \mathrm{~L})$ to give 12 fractions: Fr. 1 (105 g), Fr. 2 (2.5 g), Fr. 3 (1.6 g), Fr. 4 (3.2 g), Fr. 5 (4.5 g), Fr. 6 (5.6 g), Fr. 7 (5.0 g), Fr. 8 (2.2 g), Fr. 9 (1.2 g), Fr. 10 (1.1 g), Fr. 11 (1.7 g), and Fr. 12 (3.8 g). Fr. 1 was separated by Sephadex LH-20 (4 cm i.d. $\times 25$ cm, $0-100 \% \mathrm{MeOH}$ in $\left.\mathrm{H}_{2} \mathrm{O}\right)$ into seven fractions: 1a-1g. Fr. 1a (100g) was dissolved in 0.1 $\mathrm{mol} / \mathrm{L} \mathrm{HCl}$ to convert the carboxylic acid salts into free carboxylic acids, and then fractionated by Sephadex LH-20 (4 cm i.d. $\times 25 \mathrm{~cm}, 0-70 \% \mathrm{MeOH}$ in $\left.\mathrm{H}_{2} \mathrm{O}\right)$ to give six fractions (1a1-1a6). Fr. 1a3 was identified as chebulic acid (7) (438.0 mg). Fr. 1a4 was further separated by Toyopearl butyl-650C (0-80\% $\mathrm{MeOH})$ into seven fractions, and Fr. 1a4d was further separated by Sephadex LH-20 chromatography $\left(0-60 \% \mathrm{MeOH}\right.$ in $\left.\mathrm{H}_{2} \mathrm{O}\right)$ to give $2(27 \mathrm{mg})$. Fr. 1c $(841 \mathrm{mg})$ was applied to a Sephadex LH-20 column $\left(3 \mathrm{~cm}\right.$ i.d. $\times 20 \mathrm{~cm}, 0-70 \% \mathrm{MeOH}$ in $\left.\mathrm{H}_{2} \mathrm{O}\right)$ to yield phyllaembrinin F (6) (131.3 mg). Fr. 4 (3.2 g) was fractionated by Sephadex LH-20 (4 cm i.d. × $25 \mathrm{~cm}, 0-100 \% \mathrm{MeOH})$ into 10 fractions: 4a-4r. Fr. 4h (100 mg) was separated by Sephadex LH-20 (100-50\% EtOH in $\mathrm{H}_{2} \mathrm{O}$ ) to yield 1 (77 mg). Fr. 10 was separated by Sephadex LH-20 (4 
cm i.d. $\times 27 \mathrm{~cm}, 30-100 \% \mathrm{MeOH}$ in $\mathrm{H}_{2} \mathrm{O}$, then $\mathrm{MeOH}$ containing increasing proportions of $60 \%$ aq. acetone; 1:9-3:7) to yield 3 (32 mg). Fr. 11 (1.7 g) was subjected to Sephadex LH-20 column chromatography $(3 \mathrm{~cm}$ i.d. $\times 24 \mathrm{~cm})$ and eluted by $20-100 \% \mathrm{MeOH}$ in $\mathrm{H}_{2} \mathrm{O}$ to give eight fractions: 11a-11h. Fr. 11d (245 mg) was separated by chromatography using Chromatorex ODS (0-70\% $\mathrm{MeOH}$ in $\left.\mathrm{H}_{2} \mathrm{O}\right)$, Toyopearl HW 40F (30-100\% MeOH in $\mathrm{H}_{2} \mathrm{O}$ ), Diaion HP20SS (50$100 \% \mathrm{MeOH}$ in $\left.\mathrm{H}_{2} \mathrm{O}\right)$, and Sephadex $\mathrm{LH}-20\left(0-100 \% \mathrm{MeOH}\right.$ in $\left.\mathrm{H}_{2} \mathrm{O}\right)$ to yield 5 (17.7 mg). Fr. 11e (453 mg) was fractionated by Wakogel $100 \mathrm{C}_{18}\left(10-100 \% \mathrm{MeOH}\right.$ in $\left.\mathrm{H}_{2} \mathrm{O}\right)$ and BondaPak $\mathrm{C}_{18}$ (30-100\% $\mathrm{MeOH}$ in $\mathrm{H}_{2} \mathrm{O}$ ) to afford 3 (24 mg).

Penthorumnin A (1). Brown amorphous powder, $[\alpha]_{\mathrm{D}}-18(c$ 0.2, $\mathrm{MeOH})$; UV $(\mathrm{MeOH}) \lambda_{\max }(\log \varepsilon): 218$ (4.74), 279 (4.25); IR $v_{\max } \mathrm{cm}^{-1}: 3390,1721,1615,1327$; ESIMS m/z: $651[\mathrm{M}-\mathrm{H}]^{-}, 607\left[\mathrm{M}-\mathrm{CO}_{2} \mathrm{H}\right]^{-}$; HR-ESI-MS m/z: $651.0848[\mathrm{M}-\mathrm{H}]^{-}$(calcd. for $^{2}$ $\left.\mathrm{C}_{27} \mathrm{H}_{23} \mathrm{O}_{19}, 651.0834\right) ;{ }^{1} \mathrm{H}-\mathrm{NMR}$ (methanol- $d_{4}, 500 \mathrm{MHz}$ ) and ${ }^{13} \mathrm{C}$ NMR (methanol- $d_{4}, 125$ MHz) see Table 1; ECD (MeOH) $\Delta \varepsilon(n m):-13.21(220),+3.48$ (261).

Penthorumnin $B$ (2). Brown amorphous solid, $[\alpha]_{\mathrm{D}}-27$ (c $\left.0.1, \mathrm{MeOH}\right)$; UV $(\mathrm{MeOH}) \lambda_{\max }(\log \varepsilon): 287$ (4.54), 220 (4.70); IR $v_{\max } \mathrm{cm}^{-1}: 3345,1707,1663$, 1617; ESIMS (negative) $m / z: 281[\mathrm{M}-\mathrm{H}]^{-} ; 237\left[\mathrm{M}-\mathrm{H}-\mathrm{CO}_{2}\right]^{-} ; 193\left[\mathrm{M}-\mathrm{H}-2 \mathrm{CO}_{2}\right]^{-} ; \mathrm{HR}-\mathrm{FAB}-\mathrm{MS}$ $m / z: 283.0458[\mathrm{M}+\mathrm{H}]^{+}\left(\right.$calcd for $\left.\mathrm{C}_{12} \mathrm{H}_{11} \mathrm{O}_{8}, 283.0458\right) ;{ }^{1} \mathrm{H}$ NMR (methanol- $d_{4}, 500 \mathrm{MHz}$ ) $\delta 6.74(1 \mathrm{H}, \mathrm{s}, \mathrm{H} 4), 3.96(1 \mathrm{H}, \mathrm{d}, J=4.1 \mathrm{~Hz}, \mathrm{H} 1), 3.05(1 \mathrm{H}, \mathrm{dt}, J=4.3,7.3 \mathrm{~Hz}, \mathrm{H} 2), 2.86(1 \mathrm{H}, \mathrm{dd}$, $J=4.3,17.2 \mathrm{~Hz}, \mathrm{H} 9 \mathrm{a}), 2.75(1 \mathrm{H}, \mathrm{dd}, J=7.3,17.2 \mathrm{~Hz}, \mathrm{H} 9 \mathrm{~b}) ;{ }^{13} \mathrm{C}$ NMR (methanol- $d 4,125$ MHz) $\delta 206.1$ (C3), 177.6 (C8), 175.0 (C10), 148.5 (C6), 143.5 (C5), 142.6 (C7), 132.3 (C3a), 128.2 (C7a), 101.7 (C4), 49.8 (C2), 49.5 (C1), 35.1 (C9); ECD (MeOH) $\Delta \varepsilon(\mathrm{nm}):-1.02$ (230), $-0.61(252),-0.80(273)$. 
Penthorumnin $C$ (3). Brown amorphous powder, $[\alpha]_{\mathrm{D}}+3(c 0.1, \mathrm{MeOH})$; UV $(\mathrm{MeOH}) \lambda_{\max }(\log \varepsilon): 278$ (4.42), 224 (4.53), 209 (4.52); IR $v_{\max } \mathrm{cm}^{-1}: 3422,1804,1724$, 1621. FAB-MS $m / z: 621[\mathrm{M}+\mathrm{H}]^{+}$; HR-FAB-MS $m / z: 621.1091[\mathrm{M}+\mathrm{H}]^{+}$(calcd for $\mathrm{C}_{27} \mathrm{H}_{25} \mathrm{O}_{17}, 621.1092$ ); ${ }^{1} \mathrm{H}-\mathrm{NMR}$ (acetone- $d_{6}, 500 \mathrm{MHz}$ ) and ${ }^{13} \mathrm{C}$ NMR (acetone- $d_{6}, 125$ MHz) see Table 3.

Penthorumnin $D$ (5). Yellow amorphous powder, $[\alpha]_{\mathrm{D}}+53(c$ $0.1, \mathrm{MeOH})$; UV $(\mathrm{MeOH}) \lambda_{\max }(\log \varepsilon): 330$ (3.59), 277 (4.32), 221 (4.58); IR $v_{\max } \mathrm{cm}^{-1}: 3397,1709,1623$; FAB-MS $m / z: 649[\mathrm{M}+\mathrm{H}]^{+}$; HR-FAB-MS $m / z: 649.1014[\mathrm{M}+\mathrm{H}]^{+}$(calcd for $\mathrm{C}_{28} \mathrm{H}_{25} \mathrm{O}_{18}$, 649.1041); ${ }^{1} \mathrm{H}-\mathrm{NMR}$ (acetone- $d_{6}, 400 \mathrm{MHz}$ ) and ${ }^{13} \mathrm{C}$ NMR (acetone- $d_{6}, 100 \mathrm{MHz}$ ) see Table 4; ECD (MeOH) $\Delta \varepsilon(n m):-29.03(207),+16.24(236),-9.36(270)$.

Reduction of 5. An aqueous solution of dithiothreitol (60 $\mathrm{mg}$ in $3 \mathrm{~mL}$ ) was added dropwise to an ethanolic solution of $5(15 \mathrm{mg} / 0.5 \mathrm{~mL})$ until the yellow color disappeared. The mixture was separated by Sephadex LH-20 column chromatography (1 $\times 10 \mathrm{~cm}, 0-100 \% \mathrm{MeOH})$ to yield $\mathbf{5 a}(2.2 \mathrm{mg})$.

Computational Methods. A conformational search was performed using the Monte Carlo method and the MMFF94 force field with Spartan '14 (Wavefunction, Irvine, CA). The obtained low-energy conformers within a $6 \mathrm{kcal} / \mathrm{mol}$ window were optimized at the B3LYP/631G(d,p) level (PCM) (1 and 3) or at the B3LYP/6-311+G(2d,p) level (PCM) (2). The vibrational frequencies were calculated at the same level to confirm their stability, and no imaginary frequencies were found. The ${ }^{1} \mathrm{H}$ and ${ }^{13} \mathrm{C}$ NMR chemical shifts of the low-energy conformers with Boltzmann populations greater than $1 \%$ were calculated using the gaugeincluding atomic orbital (GIAO) method at the mPW1PW91/6-311+G(2d,p) level (PCM). ${ }^{13}$ Calculated NMR chemical shifts were linearly corrected for the experimental data. ${ }^{1} \mathrm{H}$ NMR 
coupling constants of the low-energy conformers were calculated at the B3LYP/6-31G(d,p)u+1s (using only the Fermi contact term) level (PCM) and scaled by using the slope parameter $0.94 .{ }^{14}$ The energies, oscillator strengths, and rotational strengths of the low-energy conformers were calculated using TDDFT at the CAM-B3LYP/6-31G(d,p) level (PCM) (1 and 3) or at the BH\&HLYP/6-311+G(2d,p) level (PCM) (2). ${ }^{15}$ The ECD spectra were simulated by the overlapping Gaussian function with a $0.35 \mathrm{eV}(\mathbf{1}), 0.60 \mathrm{eV}(\mathbf{2})$, or $0.30 \mathrm{eV}$ (3) exponential halfwidth. Specific rotations of the low-energy conformers with Boltzmann populations larger than $1 \%$ were calculated at the B3LYP/6-311+G(2d,p) level (PCM) by using frequency dependent polarizabilities at $589.3 \mathrm{~nm}$. The calculated data for each conformer were averaged according to the Boltzmann distribution theory at $298 \mathrm{~K}$ based on their relative Gibbs free energies. All DFT calculations were performed using Gaussian 09. ${ }^{16}$ GaussView was used to draw the threedimensional molecular structures. ${ }^{17}$

\section{ASSOCIATED CONTENT}

Supporting Information. ${ }^{1} \mathrm{H}$ NMR, ${ }^{13} \mathrm{C}$ NMR, and 2D NMR spectra of $\mathbf{1 - 3}$ and $\mathbf{5}$, and computational methods and results are available in the supporting information. This material is available free of charge online at http://pubs.acs.org.

\section{AUTHOR INFORMATION}

\section{Corresponding Author.}

*Tel.: +81-95-819-2432. E-mail: t-tanaka@nagasaki-u.ac.jp.

\section{ORCID}

Yosuke Matsuo: 0000-0002-6462-9727 
Yoshinori Saito: 0000-0002-3587-9743

Takashi Tanaka: 0000-0001-7762-7432

\section{Author Contributions}

M. Era and Y. Matsuo contributed equally.

\section{Notes}

The authors declare no competing financial interest.

\section{ACKNOWLEDGMENTS}

This work was supported by the Japan Society for the Promotion of Science KAKENHI (Grant Nos. 17K08338 and 16K07741). The authors are grateful to N. Tsuda, K. Chifuku, and H. Iwata at the Center for Industry, University and Government Cooperation, Nagasaki University, for recording the NMR and MS data. The computational analysis was partly carried out using the computer facilities at the Research Institute for Information Technology, Kyushu University.

\section{REFERENCES}

(1) Yin, W.Y.; Xu, J.Y.; Zheng, W.D.; Chen, G.H.; Song, M. Food Res. Dev., 2006, 27, 147148.

(2) Zhou, S.Q.; Yin, C.Y.; Peng, L.L.; Zhou, Y.L.; Chen, C.X.; Wen, Z.J.; Jiang, G.L. Pharmacol. Clin. Chin. Mater. Med. 1987, 3, 16-20.

(3) Xia, X.D.; Chen, J.; Fan, S.; Gao, X.L.; Li, L.H.; Chen, R.J.; Zhong, K.; Gao, H.; Yamaguchi, I.; Xu, Z.J. J. Med. Plants Res. 2012, 6, 4292-4298.

(4) Cao, Y.W.; Jiang, Y.; Zhang, D.Y.; Wang, M.; Chen, H.S.; Su, H.; Wang, Y.T.; Wan, J.B. J. 
Ethnopharmacol. 2015, 161, 92-98.

(5) Wang, H.W.; Liu, Y.Q.; Feng, C.G. J. Asian Nat. Prod. Res. 2006, 8, 757-761.

(6) Huang, D.; Jiang, Y.; Chen, W.; Yao, F.; Sun, L. Molecules 2014, 19, 11045-11055.

(7) Era, M.; Matsuo, Y.; Shii, T.; Saito, Y.; Tanaka, T.; Jiang, Z.-H. J. Nat. Prod. 2015, 78, 2104-2109.

(8) Gupta, R. K.; Al-Shafi, S. M. K.; Layden, K.; Haslam, E. J. Chem. Soc., Perkin Trans. 1 1982, 2525-2534.

(9) Jiang, Z.-H.; Wen, X.-Y.; Tanaka, T.; Wu, S.-Y.; Liu, Z.; Iwata, H.; Hirose, Y.; Wu, S.; Kouno, I. J. Nat. Prod. 2008, 71, 719-723.

(10) Zhang,Y.-J.; Abe, T.; Tanaka, T.; Yang, C.-R.; Kouno, I. J. Nat. Prod. 2001, 64, 1527-1532.

(11) (a) Nonaka, G.; Harada, M.; Nishioka, I. Chem. Pharm. Bull. 1980, 28, 685-687; (b) Haddock, E.A.; Gupta, R.K.; Haslam, E. J. Chem. Soc., Perkin Trans. 1 1982, 2535-2545;

(c) Tanaka, T.; Nonaka, G.; Nishioka, I. Chem. Pharm. Bull. 1990, 38, 2424-2428.

(12) Niemetz, R.; Gross, G. G. Phytochemistry 2005, 66, 2001-2011.

(13) (a) Lodewyk, M. W.; Siebert, M. R.; Tantillo, D. J. Chem. Rev. 2012, 112, 1839-1862; (b) Willoughby, P. H.; Jansma, M. J.; Hoye, T. R. Nat. Protoc. 2014, 9, 643-660; (c) Grimblat, N.; Sarotti, A. M. Chem. Eur. J. 2016, 22, 12246-12261.

(14) Bally, T.; Rablen, P. R. J. Org. Chem. 2011, 76, 4818-4830.

(15) (a) Pescitelli, G.; Bruhn, T. Chirality 2016, 28, 466-474; (b) Nugroho, A. E.; Morita, H. J. Nat. Med. 2014, 68, 1-10; (c) Li, X.-C.; Ferreira, D.; Ding, Y. Curr. Org. Chem. 2010, 14, $1678-1697$.

(16) Frisch, M. J.; Trucks, G. W.; Schlegel, H. B.; Scuseria, G. E.; Robb, M. A.; Cheeseman, J. R.; Scalmani, G.; Barone, V.; Mennucci, B.; Petersson, G. A.; Nakatsuji, H.; Caricato, M.; 
Li, X.; Hratchian, H. P.; Izmaylov, A. F.; Bloino, J.; Zheng, G.; Sonnenberg, J. L.; Hada, M.;

Ehara, M.; Toyota, K.; Fukuda, R.; Hasegawa, J.; Ishida, M.; Nakajima, T.; Honda, Y.;

Kitao, O.; Nakai, H.; Vreven, T.; Montgomery, J. A., Jr.; Peralta, J. E.; Ogliaro, F.; Bearpark, M.; Heyd, J. J.; Brothers, E.; Kudin, K. N.; Staroverov, V. N.; Kobayashi, R.; Normand, J.; Raghavachari, K.; Rendell, A.; Burant, J. C.; Iyengar, S. S.; Tomasi, J.; Cossi, M.; Rega, N.; Millam, M. J.; Klene, M.; Knox, J. E.; Cross, J. B.; Bakken, V.; Adamo, C.; Jaramillo, J.; Gomperts, R.; Stratmann, R. E.; Yazyev, O.; Austin, A. J.; Cammi, R.; Pomelli, C.;

Ochterski, J. W.; Martin, R. L.; Morokuma, K.; Zakrzewski, V. G.; Voth, G. A.; Salvador, P.; Dannenberg, J. J.; Dapprich, S.; Daniels, A. D.; Farkas, O.; Foresman, J. B.; Ortiz, J. V.; Cioslowski, J.; Fox, D. J. Gaussian 09, Revision D.01; Gaussian, Inc.: Wallingford, CT, 2013.

(17) Dennington, R.; Keith, T.; Millam, J. GaussView, Version 5.0.9; Semichem Inc.: Shawnee Mission, KS, 2009.

(18) Foo, L. Y. Phytochemistry 1993, 33, 487-491. 
Table of Contents Graphic

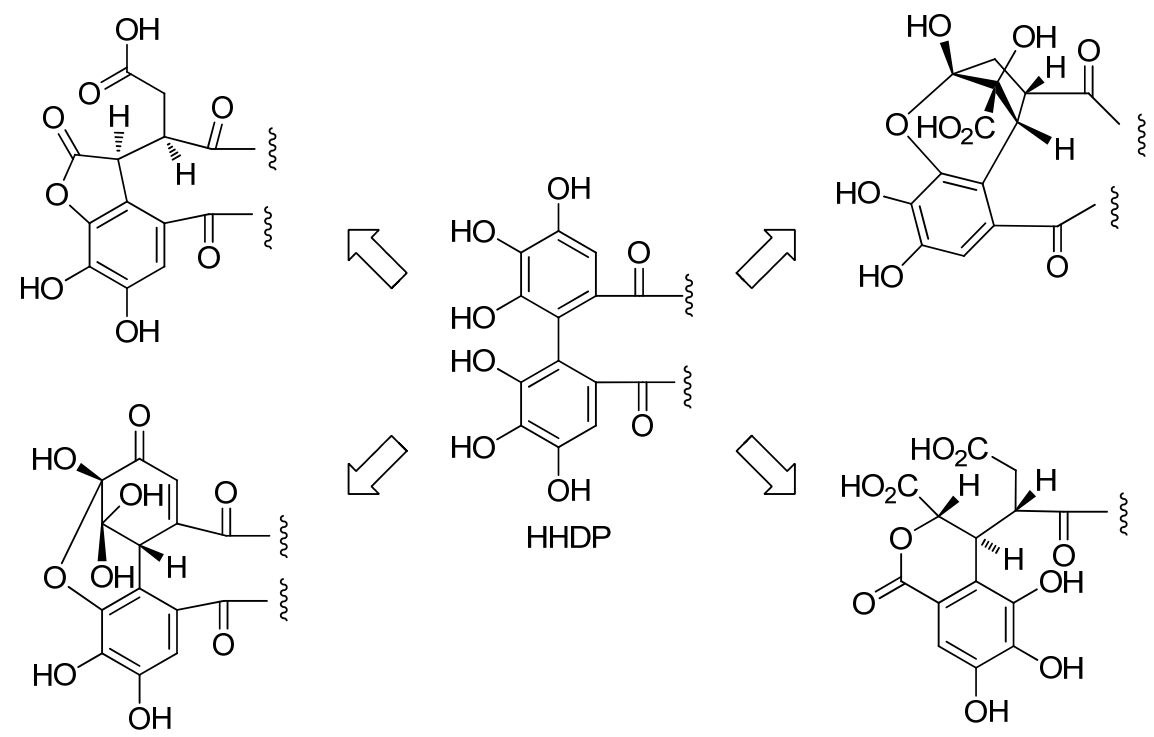




\title{
Supporting Information
}

\section{Ellagitannins and related compounds from Penthorum chinense}

\author{
Manami Era, ${ }^{\dagger}+\ddagger$ Yosuke Matsuo $, *, \dagger$ Yoshinori Saito, ${ }^{\dagger}$ Koyo Nishida,,+ Zhi-Hong Jiang ${ }^{\S}$ \\ and Takashi Tanaka, $*, \dagger$
}

\footnotetext{
${ }^{\dagger}$ Department of Natural Product Chemistry, Graduate School of Biomedical Sciences, Nagasaki University, 1-14 Bunkyo-Machi, Nagasaki 852-8521, Japan

\$Department of Pharmaceutics, Graduate School of Biomedical Sciences, Nagasaki University, 1-7-1 Sakamoto, Nagasaki 852-8501, Japan

$\S$ Institute for Applied Research in Medicine and Health, Macau University of Science and Technology, Taipa, Macau 999078, P.R. China
}

\section{Contents}

Calculation of NMR chemical shifts and ${ }^{1} \mathrm{H}$ NMR coupling constants for $\mathbf{1}$ (Figures S1-S6; Tables S1-S66)

Calculation of optical rotations for 2

(Tables S67, S68)

Calculation of NMR chemical shifts and ${ }^{1} \mathrm{H}$ NMR coupling constants for 3

(Figures S7-S10; Tables S69-S90)

Calculation of NMR chemical shifts and ${ }^{1} \mathrm{H}$ NMR coupling constants for 4

1D and 2D NMR spectra

(Figures S15-S28)

HPLC profiles

(Figures S29, S30) 
Figure S1. Possible structures of penthorumnin A (1).
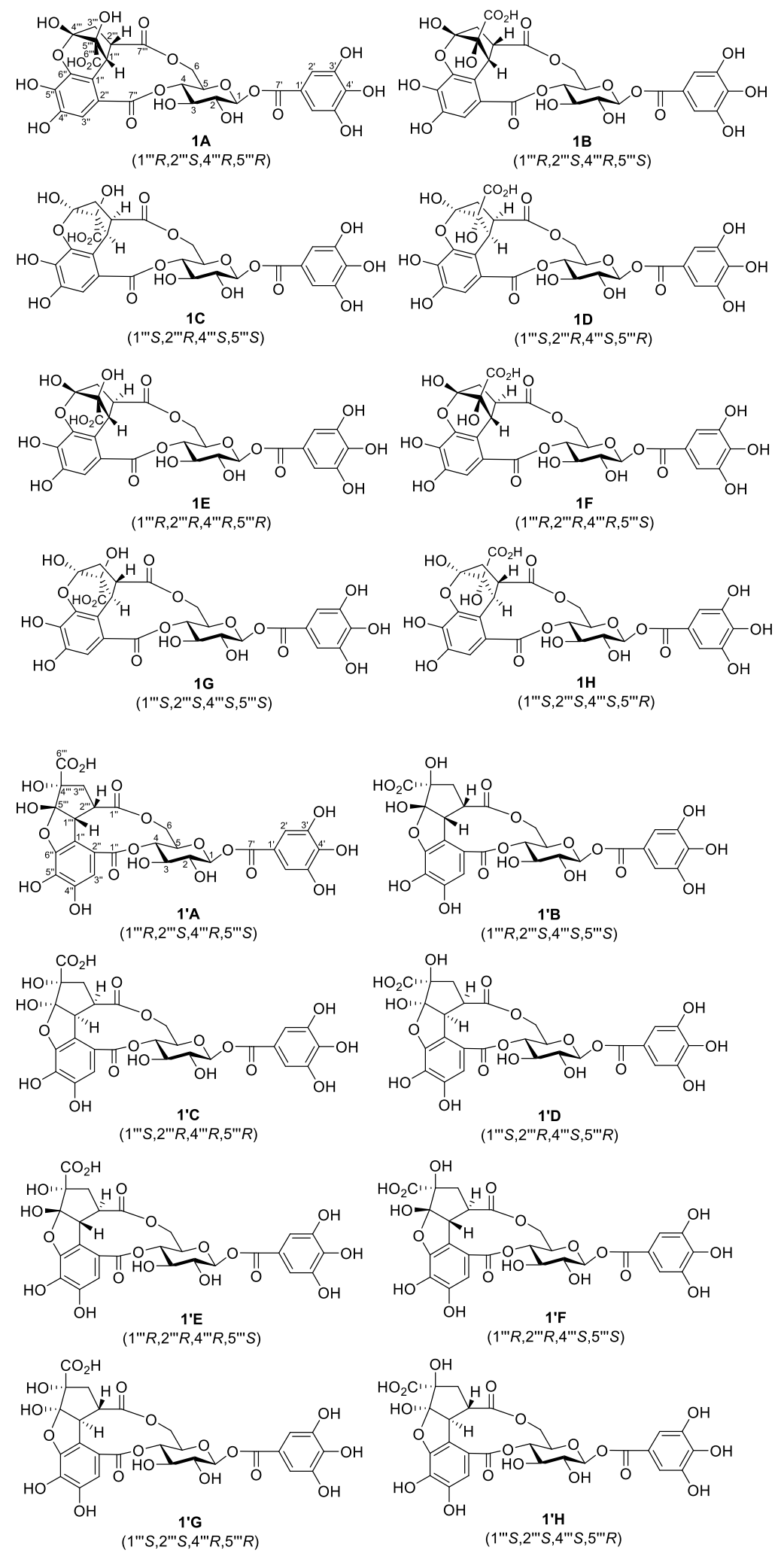
Figure S2. Lowest-energy conformers of $\mathbf{1}$ and Boltzmann-weighted calculated $J_{1^{\prime \prime \prime}-2^{\prime \prime \prime}}$ values (B3LYP/6-31G(d,p)u+1s level in MeOH (PCM)).

$J_{\text {exptl }}\left(J_{\text {calcd }}\right)$ in $\mathrm{Hz}$

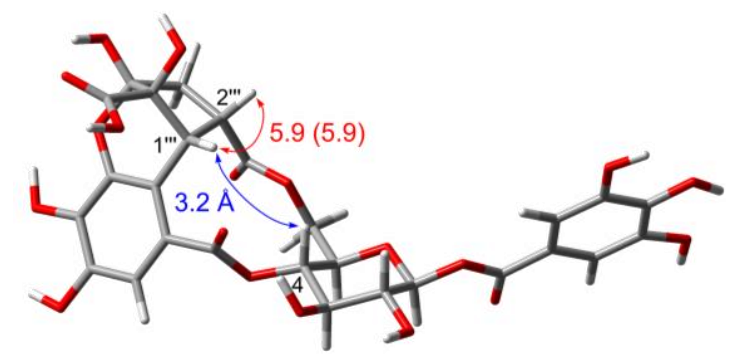

1A-1

(1'"R,2"'S,4"'R,5"'R)

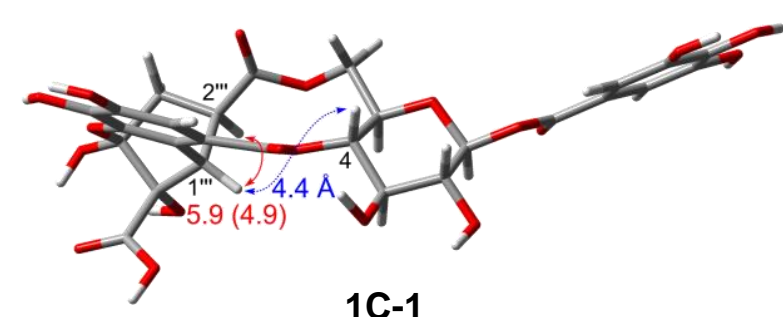

(1"'S,2'"R,4"'S,5"'S)

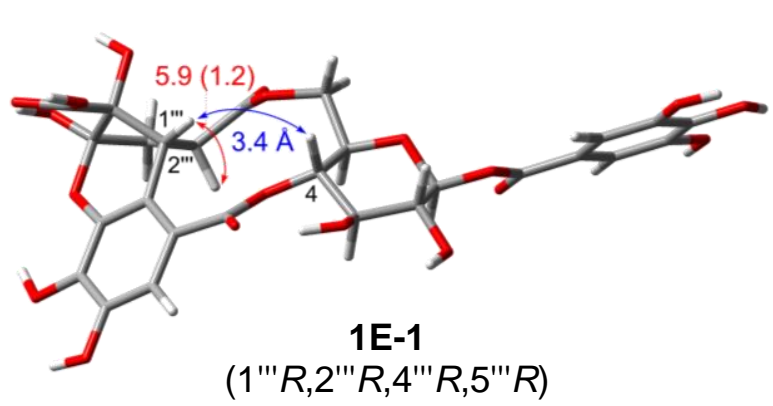

(1"'R,2'"R,4"'R,5"'R)

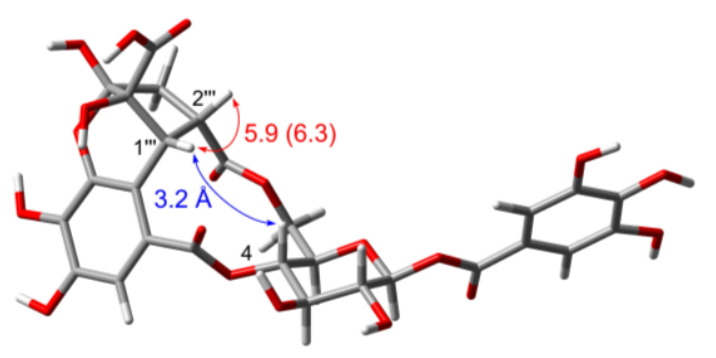

1B-1

(1"'R,2'"S,4"'R,5"'S)
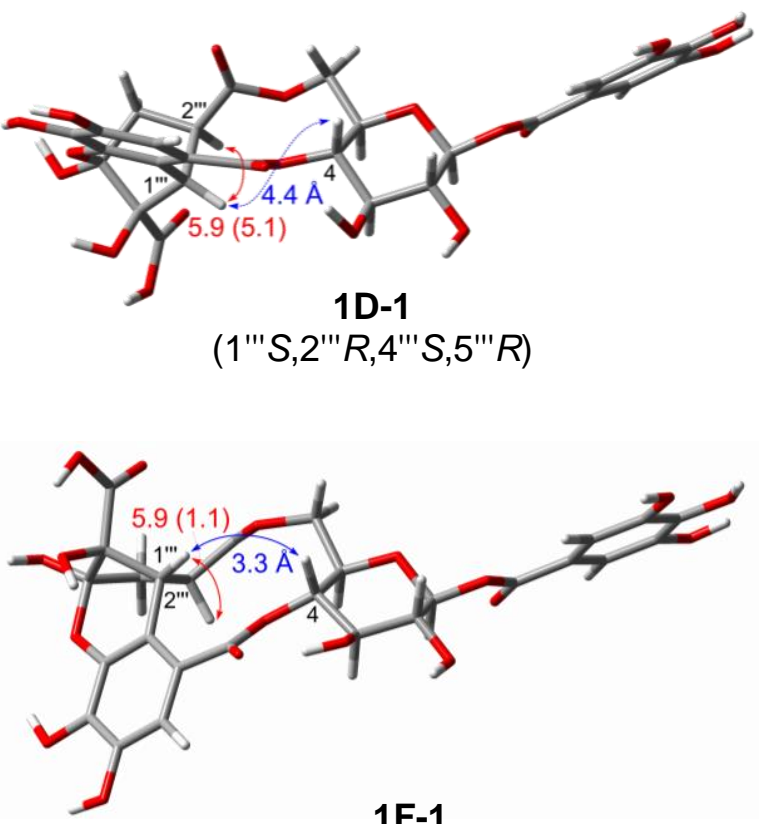

(1"'R,2"'R,4"'R,5"'S)

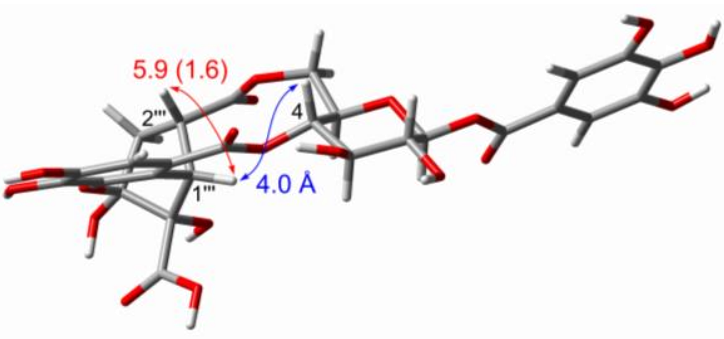

1G-1

(1"'S,2"'S,4"'S,5"'S)

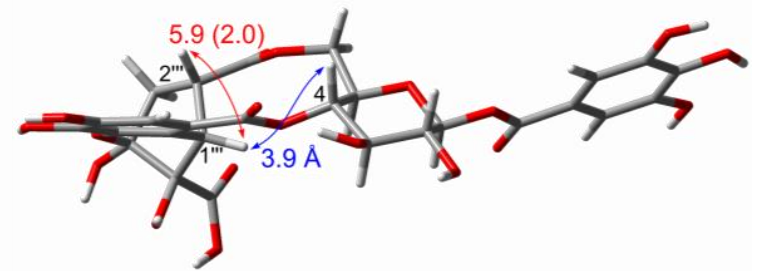

1H-1

(1"'S,2'"S,4"'S,5"'R) 
Figure S3. Lowest-energy conformers of $\mathbf{1}^{\prime}$ and Boltzmann-weighted calculated $J_{1^{\prime \prime \prime}-2^{\prime \prime \prime}}$ values (B3LYP/6-31G(d,p)u+1s level in MeOH (PCM)).

$J_{\text {exptl }}\left(J_{\text {calcd }}\right)$ in $\mathrm{Hz}$

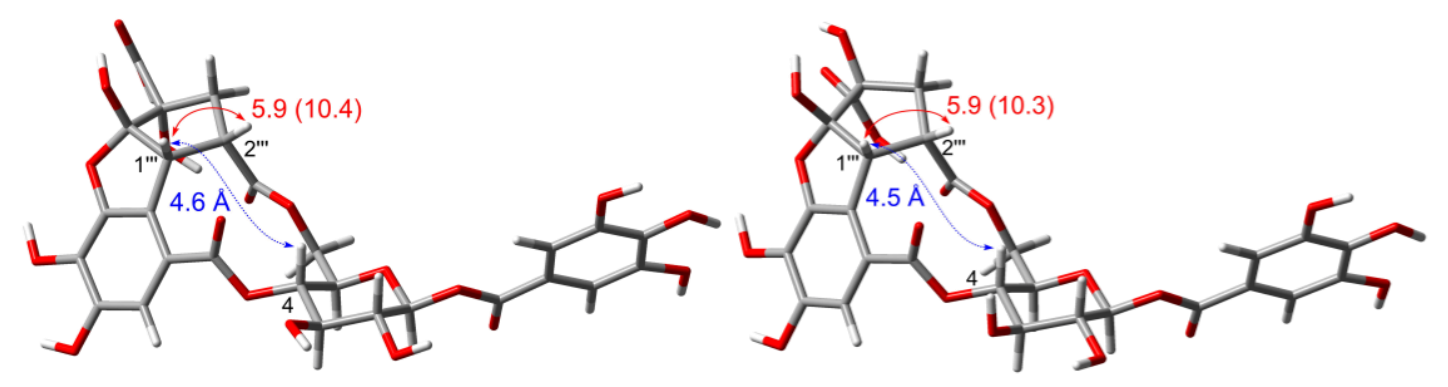

1 'A-1

(1"'R,2'"S,4"'R,5"'S)

1 'B-1

(1"'R,2"'S,4"'S,5"'S)

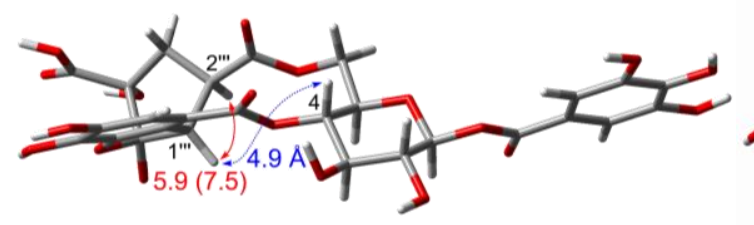

1 'C-1

(1"'S,2'" R,4"'R,5"'R)

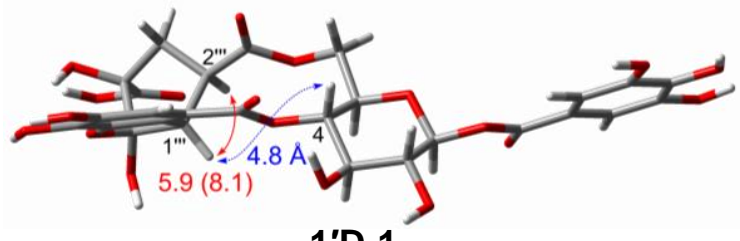

(1"'S,2"'R,4"'S,5"'R)

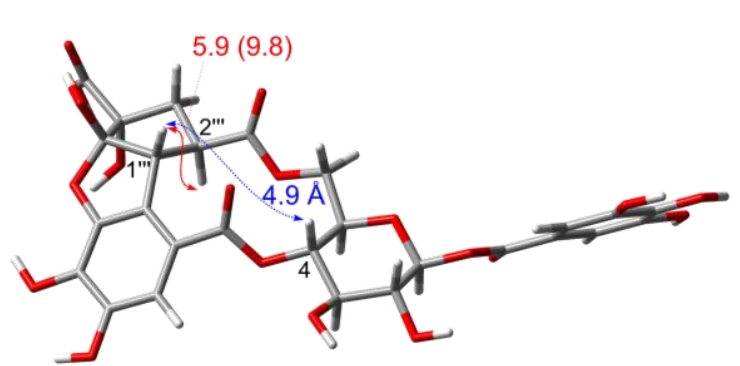

1 'E-1

(1"'R,2'"R,4"'R,5"'S)

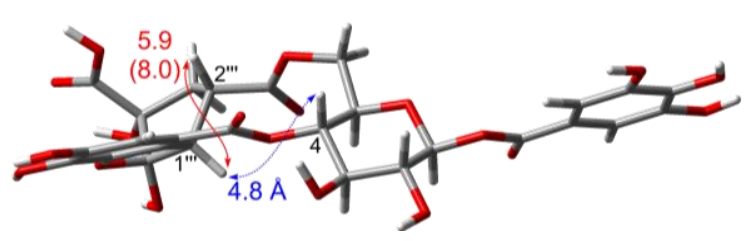

1 'G-1

(1"'S,2'"S,4"'R,5"'R)

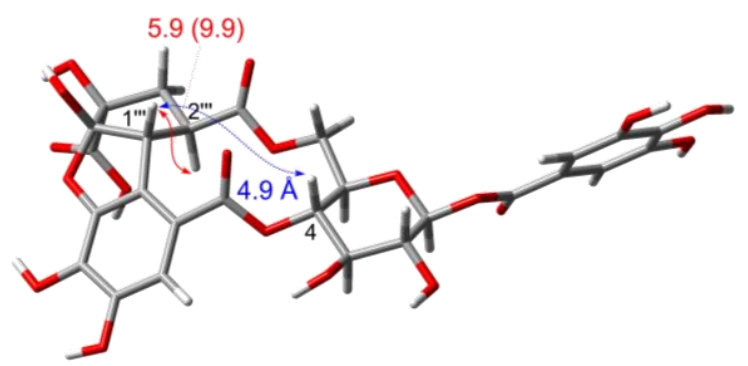

1 'F-1

(1"'R,2"'R,4"'S,5"'S)

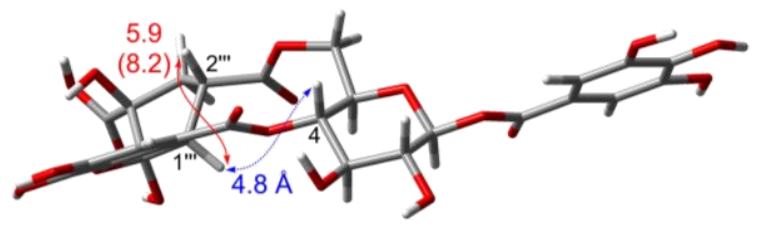

1 'H-1

(1"'S,2'"S,4"'S,5"'R) 
Table S1. Important thermodynamic parameters and conformational analysis of $\mathbf{1 A}$ at the B3LYP/6-31G(d,p) level in MeOH (PCM).

\begin{tabular}{ccccccc}
\hline conformers & $E$ (a.u.) & $E^{\prime}$ (a.u.) & $H$ (a.u.) & $G$ (a.u.) & $\Delta G(\mathrm{kca} / \mathrm{mol})$ & $\mathrm{P}_{\mathrm{G}}(\%)$ \\
\hline $\mathbf{1 A - 1}$ & -2472.658037 & -2472.141899 & -2472.099945 & -2472.217191 & 0.00 & 38.0 \\
$\mathbf{1 A - 2}$ & -2472.658035 & -2472.141861 & -2472.099930 & -2472.216771 & 0.26 & 24.3 \\
$\mathbf{1 A - 3}$ & -2472.657985 & -2472.141658 & -2472.099786 & -2472.215912 & 0.80 & 9.8 \\
$\mathbf{1 A - 4}$ & -2472.657289 & -2472.140627 & -2472.098974 & -2472.215364 & 1.15 & 5.5 \\
$\mathbf{1 A - 5}$ & -2472.656014 & -2472.140112 & -2472.098088 & -2472.214877 & 1.45 & 3.3 \\
$\mathbf{1 A - 6}$ & -2472.657226 & -2472.140463 & -2472.098852 & -2472.214810 & 1.49 & 3.0 \\
$\mathbf{1 A - 7}$ & -2472.656733 & -2472.140350 & -2472.098490 & -2472.214583 & 1.64 & 2.4 \\
$\mathbf{1 A - 8}$ & -2472.655978 & -2472.139939 & -2472.097962 & -2472.214249 & 1.85 & 1.7 \\
$\mathbf{1 A - 9}$ & -2472.656668 & -2472.140306 & -2472.098444 & -2472.214232 & 1.86 & 1.7 \\
$\mathbf{1 A - 1 0}$ & -2472.655425 & -2472.139394 & -2472.097413 & -2472.214165 & 1.90 & 1.5 \\
$\mathbf{1 A - 1 1}$ & -2472.654286 & -2472.138377 & -2472.096298 & -2472.214094 & 1.94 & 1.4 \\
\hline
\end{tabular}

E: total energy; $E$ ': total energy with zero point energy; H: enthalpy; G: Gibbs free energy; $\triangle G$ : relative Gibbs free energy at the B3LYP/6-31G(d,p) level in MeOH (PCM). $P_{G}$ : conformational distribution calculated from relative Gibbs free energy.

Table S2. Important thermodynamic parameters and conformational analysis of $\mathbf{1 B}$ at the B3LYP/6-31G(d,p) level in MeOH (PCM).

\begin{tabular}{clllllr}
\hline conformers & $E$ (a.u.) & $E^{\prime}$ (a.u.) & $H$ (a.u.) & $G$ (a.u.) & $\Delta G(\mathrm{kcal} / \mathrm{mol})$ & $\mathrm{P}_{\mathrm{G}}(\%)$ \\
\hline $\mathbf{1 B - 1}$ & -2472.655400 & -2472.139434 & -2472.097474 & -2472.213900 & 0.00 & 16.6 \\
$\mathbf{1 B - 2}$ & -2472.655345 & -2472.139245 & -2472.097333 & -2472.213531 & 0.23 & 11.2 \\
$\mathbf{1 B - 3}$ & -2472.654963 & -2472.138984 & -2472.096990 & -2472.213312 & 0.37 & 8.9 \\
$\mathbf{1 B}-4$ & -2472.654774 & -2472.138770 & -2472.096826 & -2472.213300 & 0.38 & 8.8 \\
$\mathbf{1 B}-\mathbf{5}$ & -2472.654923 & -2472.138863 & -2472.096894 & -2472.213097 & 0.50 & 7.1 \\
$\mathbf{1 B - 6}$ & -2472.654689 & -2472.138566 & -2472.096660 & -2472.212956 & 0.59 & 6.1 \\
$\mathbf{1 B - 7}$ & -2472.653565 & -2472.137859 & -2472.095835 & -2472.212382 & 0.95 & 3.3 \\
$\mathbf{1 B - 8}$ & -2472.654103 & -2472.138032 & -2472.096098 & -2472.212233 & 1.05 & 2.8 \\
$\mathbf{1 B - 9}$ & -2472.653630 & -2472.137788 & -2472.095838 & -2472.212015 & 1.18 & 2.2 \\
$\mathbf{1 B - 1 0}$ & -2472.653577 & -2472.137638 & -2472.095704 & -2472.212002 & 1.19 & 2.2 \\
$\mathbf{1 B - 1 1}$ & -2472.652863 & -2472.137135 & -2472.094994 & -2472.211988 & 1.20 & 2.2 \\
$\mathbf{1 B - 1 2}$ & -2472.653193 & -2472.136925 & -2472.095140 & -2472.211856 & 1.28 & 1.9 \\
$\mathbf{1 B - 1 3}$ & -2472.653638 & -2472.137631 & -2472.095748 & -2472.211667 & 1.40 & 1.6 \\
$\mathbf{1 B - 1 4}$ & -2472.652779 & -2472.136950 & -2472.094844 & -2472.211639 & 1.42 & 1.5 \\
$\mathbf{1 B - 1 5}$ & -2472.653595 & -2472.137183 & -2472.095527 & -2472.211558 & 1.47 & 1.4 \\
$\mathbf{1 B - 1 6}$ & -2472.652736 & -2472.137059 & -2472.095193 & -2472.211499 & 1.51 & 1.3 \\
$\mathbf{1 B - 1 7}$ & -2472.652737 & -2472.137035 & -2472.095177 & -2472.211396 & 1.57 & 1.2 \\
\hline
\end{tabular}

E: total energy; $E$ ': total energy with zero point energy; H: enthalpy; G: Gibbs free energy; $\triangle$ G: relative Gibbs free energy at the B3LYP/6-31G(d,p) level in MeOH $(P C M) . P_{G}$ : conformational distribution calculated from relative Gibbs free energy. 
Table S3. Important thermodynamic parameters and conformational analysis of $\mathbf{1 C}$ at the B3LYP/6-31G(d,p) level in MeOH (PCM).

\begin{tabular}{ccccccc}
\hline conformers & $E$ (a.u.) & $E^{\prime}$ (a.u.) & $H$ (a.u.) & $G$ (a.u.) & $\Delta G(\mathrm{kcal} / \mathrm{mol})$ & $\mathrm{P}_{\mathrm{G}}(\%)$ \\
\hline $\mathbf{1 C}-1$ & -2472.661689 & -2472.145101 & -2472.103432 & -2472.219466 & 0.00 & 45.3 \\
$\mathbf{1 C}-2$ & -2472.661631 & -2472.144939 & -2472.103337 & -2472.218584 & 0.55 & 17.8 \\
$\mathbf{1 C}-3$ & -2472.660107 & -2472.143883 & -2472.102088 & -2472.218483 & 0.62 & 16.0 \\
$\mathbf{1 C}-4$ & -2472.660052 & -2472.143725 & -2472.102006 & -2472.217484 & 1.24 & 5.5 \\
$\mathbf{1 C}-5$ & -2472.659873 & -2472.143417 & -2472.101708 & -2472.217306 & 1.36 & 4.6 \\
$\mathbf{1 C}-6$ & -2472.659093 & -2472.142667 & -2472.100962 & -2472.216945 & 1.58 & 3.1 \\
$\mathbf{1 C}-\mathbf{7}$ & -2472.657731 & -2472.141691 & -2472.099826 & -2472.216603 & 1.80 & 2.2 \\
$\mathbf{1 C}-\mathbf{8}$ & -2472.659038 & -2472.142561 & -2472.100897 & -2472.216404 & 1.92 & 1.8 \\
\hline
\end{tabular}

E: total energy; $E^{\prime}$ : total energy with zero point energy; H: enthalpy; G: Gibbs free energy; $\Delta G$ : relative Gibbs free energy at the B3LYP/6-31G(d,p) level in MeOH (PCM). $P_{G}$ : conformational distribution calculated from relative Gibbs free energy.

Table S4. Important thermodynamic parameters and conformational analysis of $\mathbf{1 D}$ at the B3LYP/6-31G(d,p) level in MeOH (PCM).

\begin{tabular}{clllllr}
\hline conformers & $E$ (a.u.) & $E^{\prime}$ (a.u.) & $H$ (a.u.) & $G$ (a.u.) & $\Delta G(\mathrm{kcal} / \mathrm{mol})$ & $\mathrm{P}_{\mathrm{G}}(\%)$ \\
\hline 1D-1 & -2472.657788 & -2472.141550 & -2472.099872 & -2472.215663 & 0.00 & 41.6 \\
1D-2 & -2472.657884 & -2472.141512 & -2472.099895 & -2472.215175 & 0.31 & 24.8 \\
1D-3 & -2472.657365 & -2472.140896 & -2472.099339 & -2472.214550 & 0.70 & 12.8 \\
$\mathbf{1 D}-4$ & -2472.657431 & -2472.140850 & -2472.099344 & -2472.214140 & 0.96 & 8.3 \\
$\mathbf{1 D}-5$ & -2472.655305 & -2472.139154 & -2472.097440 & -2472.212992 & 1.68 & 2.5 \\
$\mathbf{1 D}-6$ & -2472.653996 & -2472.138103 & -2472.096174 & -2472.212684 & 1.87 & 1.8 \\
1D-7 & -2472.655367 & -2472.139108 & -2472.097441 & -2472.212610 & 1.92 & 1.6 \\
1D-8 & -2472.654074 & -2472.138083 & -2472.096197 & -2472.212377 & 2.06 & 1.3 \\
1D-9 & -2472.654306 & -2472.138165 & -2472.096381 & -2472.212183 & 2.18 & 1.0 \\
\hline
\end{tabular}

E: total energy; E': total energy with zero point energy; H: enthalpy; G: Gibbs free energy; $\triangle$ G: relative Gibbs free energy at the B3LYP/6-31G(d,p) level in $\mathrm{MeOH}(\mathrm{PCM}) . \mathrm{P}_{G}$ : conformational distribution calculated from relative Gibbs free energy. 
Table S5. Important thermodynamic parameters and conformational analysis of $\mathbf{1 E}$ at the B3LYP/6-31G(d,p) level in MeOH (PCM).

\begin{tabular}{cllllll}
\hline conformers & $E$ (a.u.) & $E^{\prime}$ (a.u.) & $H$ (a.u.) & $G$ (a.u.) & $\Delta G(\mathrm{kcal} / \mathrm{mol})$ & $\mathrm{P}_{\mathrm{G}}(\%)$ \\
\hline $\mathbf{1 E}-1$ & -2472.653735 & -2472.137527 & -2472.095889 & -2472.211801 & 0.00 & 14.7 \\
$\mathbf{1 E - 2}$ & -2472.653774 & -2472.137355 & -2472.095812 & -2472.211745 & 0.04 & 13.9 \\
$\mathbf{1 E - 3}$ & -2472.653725 & -2472.137350 & -2472.095785 & -2472.211714 & 0.05 & 13.4 \\
$\mathbf{1 E}-4$ & -2472.653313 & -2472.137210 & -2472.095454 & -2472.211678 & 0.08 & 12.9 \\
$\mathbf{1 E}-\mathbf{5}$ & -2472.653684 & -2472.137446 & -2472.095830 & -2472.211535 & 0.17 & 11.1 \\
$\mathbf{1 E - 6}$ & -2472.653368 & -2472.137138 & -2472.095438 & -2472.211448 & 0.22 & 10.1 \\
$\mathbf{1 E}-7$ & -2472.651588 & -2472.135865 & -2472.094047 & -2472.210927 & 0.55 & 5.8 \\
$\mathbf{1 E - 8}$ & -2472.651549 & -2472.135762 & -2472.093987 & -2472.210368 & 0.90 & 3.2 \\
$\mathbf{1 E - 9}$ & -2472.650563 & -2472.134980 & -2472.093006 & -2472.210275 & 0.96 & 2.9 \\
$\mathbf{1 E - 1 0}$ & -2472.650605 & -2472.134925 & -2472.092993 & -2472.210175 & 1.02 & 2.6 \\
$\mathbf{1 E - 1 1}$ & -2472.651413 & -2472.135171 & -2472.093549 & -2472.209897 & 1.19 & 2.0 \\
$\mathbf{1 E - 1 2}$ & -2472.651382 & -2472.135176 & -2472.093546 & -2472.209802 & 1.25 & 1.8 \\
$\mathbf{1 E - 1 3}$ & -2472.649204 & -2472.133765 & -2472.091684 & -2472.209477 & 1.46 & 1.3 \\
$\mathbf{1 E}-14$ & -2472.649143 & -2472.133761 & -2472.091658 & -2472.209455 & 1.47 & 1.2 \\
\hline
\end{tabular}

E: total energy; $E$ ': total energy with zero point energy; H: enthalpy; G: Gibbs free energy; $\triangle G$ : relative Gibbs free energy at the B3LYP/6-31G(d,p) level in MeOH (PCM). $P_{G}$ : conformational distribution calculated from relative Gibbs free energy.

Table S6. Important thermodynamic parameters and conformational analysis of $\mathbf{1 F}$ at the B3LYP/6-31G(d,p) level in MeOH (PCM).

\begin{tabular}{cllllll}
\hline conformers & $E$ (a.u.) & $E^{\prime}$ (a.u.) & $H$ (a.u.) & $G$ (a.u.) & $\Delta G(\mathrm{kcal} / \mathrm{mol})$ & $\mathrm{P}_{\mathrm{G}}(\%)$ \\
\hline $\mathbf{1 F - 1}$ & -2472.651244 & -2472.135533 & -2472.093782 & -2472.209602 & 0.00 & 17.3 \\
$\mathbf{1 F - 2}$ & -2472.651326 & -2472.135523 & -2472.093805 & -2472.209386 & 0.14 & 13.8 \\
$\mathbf{1 F - 3}$ & -2472.650694 & -2472.135028 & -2472.093230 & -2472.209321 & 0.18 & 12.9 \\
$\mathbf{1 F - 4}$ & -2472.650756 & -2472.135037 & -2472.093257 & -2472.209167 & 0.27 & 10.9 \\
$\mathbf{1 F - 5}$ & -2472.650726 & -2472.134944 & -2472.093254 & -2472.208952 & 0.41 & 8.7 \\
$\mathbf{1 F - 6}$ & -2472.650796 & -2472.134891 & -2472.093251 & -2472.208580 & 0.64 & 5.9 \\
$\mathbf{1 F - 7}$ & -2472.649507 & -2472.134019 & -2472.092092 & -2472.208559 & 0.65 & 5.7 \\
$\mathbf{1 F - 8}$ & -2472.649579 & -2472.134012 & -2472.092113 & -2472.208357 & 0.78 & 4.6 \\
$\mathbf{1 F - 9}$ & -2472.648847 & -2472.133429 & -2472.091484 & -2472.207948 & 1.04 & 3.0 \\
$\mathbf{1 F - 1 0}$ & -2472.648929 & -2472.133367 & -2472.091481 & -2472.207530 & 1.30 & 1.9 \\
$\mathbf{1 F - 1 1}$ & -2472.649994 & -2472.133809 & -2472.092256 & -2472.207403 & 1.38 & 1.7 \\
$\mathbf{1 F - 1 2}$ & -2472.650068 & -2472.133836 & -2472.092295 & -2472.207305 & 1.44 & 1.5 \\
$\mathbf{1 F - 1 3}$ & -2472.648426 & -2472.132994 & -2472.091125 & -2472.207126 & 1.55 & 1.3 \\
$\mathbf{1 F - 1 4}$ & -2472.648345 & -2472.132198 & -2472.090443 & -2472.206999 & 1.63 & 1.1 \\
$\mathbf{1 F - 1 5}$ & -2472.647917 & -2472.132710 & -2472.090666 & -2472.206967 & 1.65 & 1.1 \\
$\mathbf{1 F - 1 6}$ & -2472.646731 & -2472.131706 & -2472.089378 & -2472.206918 & 1.68 & 1.0 \\
\hline
\end{tabular}

E: total energy; $E^{\prime}$ : total energy with zero point energy; H: enthalpy; G: Gibbs free energy; $\triangle$ G: relative Gibbs free energy at the B3LYP/6-31G(d,p) level in MeOH $(P C M) . P_{G}$ : conformational distribution calculated from relative Gibbs free energy. 
Table S7. Important thermodynamic parameters and conformational analysis of $\mathbf{1 G}$ at the B3LYP/6-31G(d,p) level in MeOH (PCM).

\begin{tabular}{|c|c|c|c|c|c|c|}
\hline conformers & $E$ (a.u.) & $E^{\prime}$ (a.u.) & $H$ (a.u.) & $G$ (a.u.) & $\Delta G(\mathrm{kcal} / \mathrm{mol})$ & $\mathrm{P}_{\mathrm{G}}(\%)$ \\
\hline 1G-1 & -2472.657745 & -2472.141511 & -2472.099779 & -2472.215617 & 0.00 & 37.4 \\
\hline 1G-2 & -2472.657826 & -2472.141454 & -2472.099776 & -2472.215349 & 0.17 & 28.2 \\
\hline 1G-3 & -2472.655749 & -2472.139829 & -2472.097971 & -2472.214026 & 1.00 & 6.9 \\
\hline 1G-4 & -2472.655240 & -2472.139418 & -2472.097518 & -2472.214019 & 1.00 & 6.9 \\
\hline 1G-5 & -2472.655816 & -2472.139798 & -2472.097969 & -2472.213910 & 1.07 & 6.1 \\
\hline 1G-6 & -2472.655315 & -2472.139392 & -2472.097537 & -2472.213766 & 1.16 & 5.3 \\
\hline 1G-7 & -2472.654334 & -2472.138352 & -2472.096313 & -2472.212759 & 1.79 & 1.8 \\
\hline 1G-8 & -2472.653556 & -2472.137930 & -2472.095946 & -2472.212508 & 1.95 & 1.4 \\
\hline 1G-9 & -2472.653622 & -2472.137912 & -2472.095950 & -2472.212441 & 1.99 & 1.3 \\
\hline
\end{tabular}

E: total energy; $E^{\prime}$ : total energy with zero point energy; H: enthalpy; G: Gibbs free energy; $\triangle G$ : relative Gibbs free energy at the B3LYP/6-31G(d,p) level in MeOH (PCM). $P_{G}$ : conformational distribution calculated from relative Gibbs free energy.

Table S8. Important thermodynamic parameters and conformational analysis of $\mathbf{1 H}$ at the B3LYP/6-31G(d,p) level in MeOH (PCM).

\begin{tabular}{|c|c|c|c|c|c|c|}
\hline conformers & $E$ (a.u.) & $E^{\prime}$ (a.u.) & $H$ (a.u.) & $G$ (a.u.) & $\Delta G(\mathrm{kcal} / \mathrm{mol})$ & $\mathrm{P}_{\mathrm{G}}(\%)$ \\
\hline 1H-1 & -2472.652059 & -2472.136631 & -2472.094683 & -2472.211490 & 0.00 & 18.1 \\
\hline $1 \mathrm{H}-2$ & -2472.652536 & -2472.136816 & -2472.095024 & -2472.211235 & 0.16 & 13.8 \\
\hline $1 \mathrm{H}-3$ & -2472.651980 & -2472.136424 & -2472.094520 & -2472.211029 & 0.29 & 11.1 \\
\hline $1 \mathrm{H}-4$ & -2472.652730 & -2472.136607 & -2472.094813 & -2472.210977 & 0.32 & 10.5 \\
\hline $1 \mathrm{H}-5$ & -2472.652510 & -2472.136657 & -2472.094905 & -2472.210830 & 0.41 & 9.0 \\
\hline $1 H-6$ & -2472.650962 & -2472.135593 & -2472.093547 & -2472.210747 & 0.47 & 8.2 \\
\hline 1H-7 & -2472.650888 & -2472.135394 & -2472.093387 & -2472.210344 & 0.72 & 5.4 \\
\hline $1 \mathrm{H}-8$ & -2472.650071 & -2472.134980 & -2472.092814 & -2472.209861 & 1.02 & 3.2 \\
\hline $1 \mathrm{H}-9$ & -2472.649517 & -2472.134575 & -2472.092308 & -2472.209797 & 1.06 & 3.0 \\
\hline 1H-10 & -2472.651404 & -2472.135448 & -2472.093597 & -2472.209790 & 1.07 & 3.0 \\
\hline 1H-11 & -2472.650671 & -2472.135112 & -2472.093111 & -2472.209643 & 1.16 & 2.6 \\
\hline 1H-12 & -2472.651308 & -2472.135352 & -2472.093520 & -2472.209459 & 1.27 & 2.1 \\
\hline $1 H-13$ & -2472.650621 & -2472.135021 & -2472.093058 & -2472.209368 & 1.33 & 1.9 \\
\hline 1H-14 & -2472.650011 & -2472.134790 & -2472.092680 & -2472.209346 & 1.35 & 1.9 \\
\hline 1H-15 & -2472.649469 & -2472.134400 & -2472.092177 & -2472.209332 & 1.35 & 1.8 \\
\hline 1H-16 & -2472.650883 & -2472.135027 & -2472.093123 & -2472.209311 & 1.37 & 1.8 \\
\hline 1H-17 & -2472.651731 & -2472.135672 & -2472.094351 & -2472.209175 & 1.45 & 1.6 \\
\hline 1H-18 & -2472.650791 & -2472.134877 & -2472.093017 & -2472.208843 & 1.66 & 1.1 \\
\hline
\end{tabular}

E: total energy; $E$ ': total energy with zero point energy; H: enthalpy; G: Gibbs free energy; $\triangle$ G: relative Gibbs free energy at the B3LYP/6-3IG(d,p) level in MeOH (PCM). $P_{G}$ : conformational distribution calculated from relative Gibbs free energy. 
Table S9. Important thermodynamic parameters and conformational analysis of $\mathbf{1}^{\prime} \mathbf{A}$ at the B3LYP/6-31G(d,p) level in MeOH (PCM).

\begin{tabular}{ccccccc}
\hline conformers & $E$ (a.u.) & $E^{\prime}$ (a.u.) & $H$ (a.u.) & $G$ (a.u.) & $\Delta G(\mathrm{kcal} / \mathrm{mol})$ & $\mathrm{P}_{\mathrm{G}}(\%)$ \\
\hline $\mathbf{1}$ 'A-1 & -2472.669070 & -2472.152496 & -2472.111098 & -2472.225640 & 0.00 & 43.8 \\
1'A-2 & -2472.668207 & -2472.151470 & -2472.110122 & -2472.224922 & 0.45 & 20.5 \\
1'A-3 & -2472.668113 & -2472.151377 & -2472.110032 & -2472.224700 & 0.59 & 16.2 \\
1'A-4 & -2472.667411 & -2472.150831 & -2472.109617 & -2472.223935 & 1.07 & 7.2 \\
1'A-5 & -2472.667324 & -2472.150765 & -2472.109551 & -2472.223912 & 1.08 & 7.0 \\
1'A-6 & -2472.665676 & -2472.149003 & -2472.107756 & -2472.222252 & 2.13 & 1.2 \\
\hline
\end{tabular}

$E$ : total energy; $E$ ': total energy with zero point energy; H: enthalpy; G: Gibbs free energy; $\Delta$ G: relative Gibbs free energy at the B3LYP/6-3lG(d,p) level in $\mathrm{MeOH}(\mathrm{PCM}) . \mathrm{P}_{G}$ : conformational distribution calculated from relative Gibbs free energy.

Table S10. Important thermodynamic parameters and conformational analysis of $\mathbf{1}$ ' $\mathbf{B}$ at the B3LYP/6-31G(d,p) level in MeOH (PCM).

\begin{tabular}{cllllll}
\hline conformers & $E$ (a.u.) & $E^{\prime}$ (a.u.) & $H$ (a.u.) & $G$ (a.u.) & $\Delta G(\mathrm{kcal} / \mathrm{mol})$ & $\mathrm{P}_{\mathrm{G}}(\%)$ \\
\hline $\mathbf{1}$ 'B-1 & -2472.666962 & -2472.150818 & -2472.109432 & -2472.224125 & 0.00 & 29.9 \\
$\mathbf{1}$ 'B-2 & -2472.666148 & -2472.150151 & -2472.108897 & -2472.223356 & 0.48 & 13.2 \\
$\mathbf{1}$ 'B-3 & -2472.666060 & -2472.150067 & -2472.108815 & -2472.223261 & 0.54 & 12.0 \\
$\mathbf{1}$ 'B-4 & -2472.660821 & -2472.145758 & -2472.103088 & -2472.222913 & 0.76 & 8.3 \\
$\mathbf{1}$ 'B-5 & -2472.662316 & -2472.146188 & -2472.104088 & -2472.222704 & 0.89 & 6.6 \\
$\mathbf{1}$ 'B-6 & -2472.663235 & -2472.147186 & -2472.105162 & -2472.222275 & 1.16 & 4.2 \\
$\mathbf{1}$ 'B-7 & -2472.662063 & -2472.145985 & -2472.103890 & -2472.221940 & 1.37 & 2.9 \\
$\mathbf{1}$ 'B-8 & -2472.659860 & -2472.144947 & -2472.102218 & -2472.221817 & 1.45 & 2.6 \\
$\mathbf{1}$ 'B-9 & -2472.660623 & -2472.145476 & -2472.102852 & -2472.221607 & 1.58 & 2.1 \\
$\mathbf{1}$ 'B-10 & -2472.662473 & -2472.146456 & -2472.104411 & -2472.221457 & 1.67 & 1.8 \\
$\mathbf{1}$ 'B-11 & -2472.659983 & -2472.144902 & -2472.102243 & -2472.221322 & 1.76 & 1.5 \\
$\mathbf{1}$ 'B-12 & -2472.661289 & -2472.145375 & -2472.103244 & -2472.221093 & 1.90 & 1.2 \\
\hline
\end{tabular}

E: total energy; $E$ ': total energy with zero point energy; H: enthalpy; G: Gibbs free energy; $\triangle G$ : relative Gibbs free energy at the B3LYP/6-31G(d,p) level in MeOH $(P C M) . P_{G}$ : conformational distribution calculated from relative Gibbs free energy. 
Table S11. Important thermodynamic parameters and conformational analysis of $\mathbf{1}^{\prime} \mathbf{C}$ at the B3LYP/6-31G(d,p) level in MeOH (PCM).

\begin{tabular}{|c|c|c|c|c|c|c|}
\hline conformers & $E$ (a.u.) & $E^{\prime}$ (a.u.) & $H$ (a.u.) & $G$ (a.u.) & $\Delta G(\mathrm{kcal} / \mathrm{mol})$ & $\mathrm{P}_{\mathrm{G}}(\%)$ \\
\hline $1^{\prime} \mathrm{C}-1$ & -2472.668214 & -2472.151831 & -2472.110145 & -2472.226308 & 0.00 & 24.6 \\
\hline $1^{\prime} \mathrm{C}-2$ & -2472.668273 & -2472.151821 & -2472.110174 & -2472.226170 & 0.09 & 21.2 \\
\hline $1^{\prime} \mathrm{C}-3$ & -2472.666851 & -2472.150247 & -2472.108536 & -2472.224891 & 0.89 & 5.5 \\
\hline $1^{\prime} \mathrm{C}-4$ & -2472.665015 & -2472.149422 & -2472.107180 & -2472.224868 & 0.90 & 5.3 \\
\hline $1^{\prime} \mathrm{C}-5$ & -2472.666154 & -2472.150220 & -2472.108205 & -2472.224795 & 0.95 & 4.9 \\
\hline $1^{\prime} \mathrm{C}-6$ & -2472.666228 & -2472.150255 & -2472.108237 & -2472.224780 & 0.96 & 4.9 \\
\hline $1^{\prime} \mathrm{C}-7$ & -2472.664956 & -2472.149346 & -2472.107110 & -2472.224711 & 1.00 & 4.5 \\
\hline $1^{\prime} \mathrm{C}-8$ & -2472.666810 & -2472.150179 & -2472.108481 & -2472.224630 & 1.05 & 4.2 \\
\hline $1^{\prime} \mathrm{C}-9$ & -2472.666175 & -2472.149914 & -2472.107959 & -2472.224602 & 1.07 & 4.0 \\
\hline $1^{\prime} \mathrm{C}-10$ & -2472.666097 & -2472.149849 & -2472.107911 & -2472.224501 & 1.13 & 3.6 \\
\hline 1'C-11 & -2472.664584 & -2472.148972 & -2472.106701 & -2472.224343 & 1.23 & 3.1 \\
\hline $1^{\prime} \mathrm{C}-12$ & -2472.664908 & -2472.149084 & -2472.107320 & -2472.224338 & 1.24 & 3.0 \\
\hline $1^{\prime} \mathrm{C}-13$ & -2472.664520 & -2472.148876 & -2472.106619 & -2472.224078 & 1.40 & 2.3 \\
\hline $1^{\prime} \mathrm{C}-14$ & -2472.664981 & -2472.148955 & -2472.107084 & -2472.223544 & 1.73 & 1.3 \\
\hline $1^{\prime} \mathrm{C}-15$ & -2472.665032 & -2472.148941 & -2472.107103 & -2472.223473 & 1.78 & 1.2 \\
\hline $1^{\prime} \mathrm{C}-16$ & -2472.664882 & -2472.148913 & -2472.107230 & -2472.223373 & 1.84 & 1.1 \\
\hline
\end{tabular}

E: total energy; $E^{\prime}$ : total energy with zero point energy; H: enthalpy; G: Gibbs free energy; $\triangle G$ : relative Gibbs free energy at the B3LYP/6-3lG(d,p) level in MeOH (PCM). $P_{G}$ : conformational distribution calculated from relative Gibbs free energy.

Table S12. Important thermodynamic parameters and conformational analysis of $\mathbf{1}^{\prime} \mathbf{D}$ at the B3LYP/6-31G(d,p) level in MeOH (PCM).

\begin{tabular}{ccccccc}
\hline conformers & $E$ (a.u.) & $E^{\prime}$ (a.u.) & $H$ (a.u.) & $G$ (a.u.) & $\Delta G(\mathrm{kcal} / \mathrm{mol})$ & $\mathrm{P}_{\mathrm{G}}(\%)$ \\
\hline 1'D-1 & -2472.666184 & -2472.149891 & -2472.108120 & -2472.225170 & 0.00 & 41.8 \\
$\mathbf{1}$ 'D-2 & -2472.666272 & -2472.149875 & -2472.108146 & -2472.224844 & 0.20 & 29.6 \\
1'D-3 & -2472.664576 & -2472.148782 & -2472.106754 & -2472.223396 & 1.11 & 6.4 \\
1'D-4 & -2472.664748 & -2472.148301 & -2472.106583 & -2472.222810 & 1.48 & 3.4 \\
1'D-5 & -2472.664828 & -2472.148271 & -2472.106564 & -2472.222721 & 1.54 & 3.1 \\
1'D-6 & -2472.664739 & -2472.148229 & -2472.106524 & -2472.222707 & 1.55 & 3.1 \\
1'D-7 & -2472.663227 & -2472.147276 & -2472.105179 & -2472.222436 & 1.72 & 2.3 \\
1'D-8 & -2472.663079 & -2472.147162 & -2472.105343 & -2472.221919 & 2.04 & 1.3 \\
1'D-9 & -2472.662906 & -2472.146939 & -2472.104956 & -2472.221801 & 2.11 & 1.2 \\
1'D-10 & -2472.662952 & -2472.146911 & -2472.104954 & -2472.221779 & 2.13 & 1.2 \\
\hline
\end{tabular}

E: total energy; $E$ ': total energy with zero point energy; H: enthalpy; G: Gibbs free energy; $\triangle$ G: relative Gibbs free energy at the B3LYP/6-31G(d,p) level in MeOH $(P C M) . P_{G}$ : conformational distribution calculated from relative Gibbs free energy. 
Table S13. Important thermodynamic parameters and conformational analysis of $\mathbf{1}^{\prime} \mathbf{E}$ at the B3LYP/6-31G(d,p) level in MeOH (PCM).

\begin{tabular}{clllllr}
\hline conformers & $E$ (a.u.) & $E^{\prime}$ (a.u.) & $H$ (a.u.) & $G$ (a.u.) & $\Delta G(\mathrm{kcal} / \mathrm{mol})$ & $\mathrm{P}_{\mathrm{G}}(\%)$ \\
\hline $\mathbf{1}$ 'E-1 & -2472.672299 & -2472.156406 & -2472.114465 & -2472.230941 & 0.00 & 27.2 \\
$\mathbf{1} ' \mathbf{E}-2$ & -2472.672142 & -2472.156256 & -2472.114310 & -2472.230672 & 0.17 & 20.5 \\
$\mathbf{1}$ 'E-3 & -2472.671971 & -2472.156036 & -2472.114133 & -2472.230493 & 0.28 & 16.9 \\
$\mathbf{1}$ 'E-4 & -2472.671919 & -2472.155962 & -2472.114075 & -2472.230293 & 0.41 & 13.7 \\
$\mathbf{1}$ 'E-5 & -2472.671653 & -2472.154967 & -2472.113240 & -2472.229164 & 1.12 & 4.1 \\
$\mathbf{1}$ 'E-6 & -2472.669308 & -2472.153555 & -2472.111352 & -2472.228732 & 1.39 & 2.6 \\
$\mathbf{1}$ 'E-7 & -2472.670641 & -2472.154576 & -2472.112886 & -2472.228416 & 1.58 & 1.9 \\
$\mathbf{1}$ 'E-8 & -2472.669166 & -2472.153305 & -2472.111162 & -2472.228299 & 1.66 & 1.7 \\
$\mathbf{1}$ 'E-9 & -2472.669102 & -2472.153222 & -2472.111101 & -2472.228100 & 1.78 & 1.3 \\
\hline
\end{tabular}

E: total energy; $E^{\prime}$ : total energy with zero point energy; H: enthalpy; G: Gibbs free energy; $\triangle$ G: relative Gibbs free energy at the B3LYP/6-31G(d,p) level in MeOH (PCM). $P_{G}$ : conformational distribution calculated from relative Gibbs free energy.

Table S14. Important thermodynamic parameters and conformational analysis of $\mathbf{1}^{\prime} \mathbf{F}$ at the B3LYP/6-31G(d,p) level in MeOH (PCM).

\begin{tabular}{clllllr}
\hline conformers & $E$ (a.u.) & $E^{\prime}$ (a.u.) & $H$ (a.u.) & $G$ (a.u.) & $\Delta G(\mathrm{kcal} / \mathrm{mol})$ & $\mathrm{P}_{\mathrm{G}}(\%)$ \\
\hline $\mathbf{1}$ 'F-1 & -2472.672529 & -2472.156974 & -2472.114875 & -2472.231815 & 0.00 & 32.1 \\
$\mathbf{1}$ 'F-2 & -2472.672488 & -2472.156876 & -2472.114801 & -2472.231563 & 0.16 & 24.5 \\
$\mathbf{1}$ 'F-3 & -2472.672688 & -2472.157105 & -2472.115013 & -2472.231458 & 0.22 & 22.0 \\
$\mathbf{1}$ 'F-4 & -2472.669166 & -2472.154157 & -2472.111680 & -2472.229741 & 1.30 & 3.6 \\
$\mathbf{1}$ 'F-5 & -2472.669548 & -2472.154150 & -2472.111831 & -2472.229478 & 1.47 & 2.7 \\
$\mathbf{1}$ 'F-6 & -2472.669090 & -2472.154026 & -2472.111571 & -2472.229446 & 1.49 & 2.6 \\
$\mathbf{1}$ 'F-7 & -2472.671220 & -2472.155360 & -2472.113509 & -2472.229328 & 1.56 & 2.3 \\
$\mathbf{1}$ 'F-8 & -2472.669638 & -2472.154311 & -2472.112068 & -2472.229161 & 1.67 & 1.9 \\
$\mathbf{1}$ 'F-9 & -2472.669270 & -2472.153881 & -2472.111578 & -2472.229012 & 1.76 & 1.6 \\
$\mathbf{1}$ 'F-10 & -2472.669588 & -2472.154207 & -2472.111985 & -2472.228912 & 1.82 & 1.5 \\
$\mathbf{1}$ 'F-11 & -2472.668652 & -2472.153518 & -2472.111124 & -2472.228840 & 1.87 & 1.4 \\
$\mathbf{1} \mathbf{1} \mathbf{F}-12$ & -2472.669213 & -2472.153762 & -2472.111487 & -2472.228709 & 1.95 & 1.2 \\
\hline
\end{tabular}

E: total energy; $E$ ': total energy with zero point energy; H: enthalpy; G: Gibbs free energy; $\Delta G$ : relative Gibbs free energy at the B3LYP/6-3IG(d,p) level in MeOH $(P C M) . P_{G}$ : conformational distribution calculated from relative Gibbs free energy. 
Table S15. Important thermodynamic parameters and conformational analysis of $\mathbf{1}^{\prime} \mathbf{G}$ at the B3LYP/6-31G(d,p) level in MeOH (PCM).

\begin{tabular}{cllllll}
\hline conformers & $E$ (a.u.) & $E^{\prime}$ (a.u.) & $H$ (a.u.) & $G$ (a.u.) & $\Delta G(\mathrm{kcal} / \mathrm{mol})$ & $\mathrm{P}_{\mathrm{G}}(\%)$ \\
\hline $\mathbf{1}$ 'G-1 & -2472.674812 & -2472.158439 & -2472.116730 & -2472.233090 & 0.00 & 26.3 \\
$\mathbf{1}$ 'G-2 & -2472.674912 & -2472.158425 & -2472.116772 & -2472.232757 & 0.21 & 18.5 \\
$\mathbf{1}$ 'G-3 & -2472.673839 & -2472.157873 & -2472.115867 & -2472.232619 & 0.30 & 16.0 \\
$\mathbf{1}$ 'G-4 & -2472.673990 & -2472.157793 & -2472.116144 & -2472.232315 & 0.49 & 11.6 \\
$\mathbf{1}$ 'G-5 & -2472.672752 & -2472.156484 & -2472.114856 & -2472.232030 & 0.67 & 8.6 \\
$\mathbf{1}$ 'G-6 & -2472.673977 & -2472.157532 & -2472.115994 & -2472.231438 & 1.04 & 4.6 \\
$\mathbf{1}$ 'G-7 & -2472.670860 & -2472.155184 & -2472.112997 & -2472.230344 & 1.72 & 1.4 \\
$\mathbf{1}$ 'G-8 & -2472.671988 & -2472.155669 & -2472.113882 & -2472.230079 & 1.89 & 1.1 \\
\hline
\end{tabular}

E: total energy; $E$ ': total energy with zero point energy; H: enthalpy; G: Gibbs free energy; $\triangle$ G: relative Gibbs free energy at the B3LYP/6-31G(d,p) level in MeOH (PCM). $P_{G}$ : conformational distribution calculated from relative Gibbs free energy.

Table S16. Important thermodynamic parameters and conformational analysis of $\mathbf{1}^{\prime} \mathbf{H}$ at the B3LYP/6-31G(d,p) level in MeOH (PCM).

\begin{tabular}{clllllr}
\hline conformers & $E$ (a.u.) & $E^{\prime}$ (a.u.) & $H$ (a.u.) & $G$ (a.u.) & $\Delta G(\mathrm{kcal} / \mathrm{mol})$ & $\mathrm{P}_{\mathrm{G}}(\%)$ \\
\hline $\mathbf{1}$ 'H-1 & -2472.674947 & -2472.157990 & -2472.116575 & -2472.232144 & 0.000 & 32.6 \\
$\mathbf{1} \mathbf{H}-2$ & -2472.674848 & -2472.157950 & -2472.116489 & -2472.232140 & 0.003 & 32.5 \\
$\mathbf{1}$ 'H-3 & -2472.673236 & -2472.156691 & -2472.115234 & -2472.231034 & 0.70 & 10.1 \\
$\mathbf{1} \mathbf{H}-4$ & -2472.673207 & -2472.156611 & -2472.115191 & -2472.230676 & 0.92 & 6.9 \\
$\mathbf{1}$ 'H-5 & -2472.671742 & -2472.155074 & -2472.113386 & -2472.229962 & 1.37 & 3.2 \\
$\mathbf{1} \mathbf{H}-\mathbf{6}$ & -2472.671831 & -2472.155092 & -2472.113459 & -2472.229807 & 1.47 & 2.7 \\
$\mathbf{1}$ 'H-7 & -2472.672322 & -2472.155429 & -2472.113963 & -2472.229559 & 1.62 & 2.1 \\
$\mathbf{1} \mathbf{H}-8$ & -2472.670418 & -2472.154043 & -2472.112338 & -2472.229239 & 1.82 & 1.5 \\
$\mathbf{1}$ 'H-9 & -2472.672280 & -2472.155310 & -2472.113887 & -2472.229007 & 1.97 & 1.2 \\
\hline
\end{tabular}

E: total energy; E': total energy with zero point energy; H: enthalpy; G: Gibbs free energy; $\Delta G$ : relative Gibbs free energy at the B3LYP/6-31G(d,p) level in $\mathrm{MeOH}(\mathrm{PCM}) . \mathrm{P}_{G}$ : conformational distribution calculated from relative Gibbs free energy. 
Table S17. Calculated ${ }^{1} \mathrm{H}$ NMR chemical shifts of $\mathbf{1 A}$.

\begin{tabular}{|c|c|c|c|c|c|c|c|c|c|c|c|c|c|c|}
\hline \multirow[b]{2}{*}{ Position } & \multicolumn{13}{|c|}{ calculated $^{a}$} & \multirow{2}{*}{$\begin{array}{c}\text { experimental }^{b} \\
1\end{array}$} \\
\hline & 1A-1 & 1A-2 & 1A-3 & 1A-4 & 1A-5 & 1A-6 & 1A-7 & 1A-8 & 1A-9 & 1A-10 & 1A-11 & $\begin{array}{c}\text { averaged- } \\
1 A^{c}\end{array}$ & $\begin{array}{l}\text { averaged-1A }^{\text {(corrected) }{ }^{d}}\end{array}$ & \\
\hline 1 & 6.12 & 6.13 & 6.13 & 6.08 & 6.14 & 6.13 & 6.14 & 6.13 & 6.13 & 6.13 & 6.14 & 6.12 & 5.71 & 5.69 \\
\hline 2 & 4.12 & 4.12 & 4.11 & 4.13 & 4.12 & 4.11 & 3.71 & 4.10 & 3.70 & 4.11 & 4.11 & 4.10 & 3.76 & 3.66 \\
\hline 3 & 4.02 & 4.02 & 4.02 & 4.29 & 4.01 & 4.31 & 4.10 & 4.01 & 4.09 & 4.01 & 4.03 & 4.05 & 3.70 & 3.77 \\
\hline 4 & 5.22 & 5.21 & 5.21 & 4.77 & 5.22 & 4.76 & 5.55 & 5.22 & 5.54 & 5.21 & 5.21 & 5.19 & 4.80 & 5.00 \\
\hline 5 & 4.35 & 4.35 & 4.34 & 4.35 & 4.36 & 4.34 & 4.21 & 4.34 & 4.20 & 4.35 & 4.37 & 4.34 & 3.99 & 3.98 \\
\hline \multirow[t]{2}{*}{6} & 5.07 & 5.07 & 5.08 & 5.09 & 5.17 & 5.12 & 5.13 & 5.17 & 5.11 & 5.03 & 4.87 & 5.08 & 4.70 & 4.64 \\
\hline & 4.03 & 4.02 & 4.03 & 3.83 & 4.01 & 3.82 & 3.97 & 4.02 & 3.96 & 4.04 & 4.12 & 4.01 & 3.66 & 3.72 \\
\hline $2^{\prime}, 6^{\prime}$ & 7.80 & 7.80 & 7.82 & 7.79 & 7.79 & 7.78 & 7.82 & 7.82 & 7.81 & 7.80 & 7.80 & 7.80 & 7.32 & 7.13 \\
\hline 3" & 6.95 & 6.95 & 6.95 & 7.36 & 6.96 & 7.36 & 7.14 & 6.96 & 7.15 & 6.90 & 7.00 & 7.00 & 6.55 & 6.59 \\
\hline $1^{\prime \prime \prime}$ & 4.53 & 4.53 & 4.53 & 3.92 & 4.52 & 3.94 & 4.80 & 4.51 & 4.80 & 4.43 & 4.45 & 4.48 & 4.13 & 4.33 \\
\hline $2^{\prime \prime \prime}$ & 3.97 & 3.97 & 3.96 & 4.15 & 3.93 & 4.14 & 4.14 & 3.92 & 4.14 & 3.95 & 4.03 & 3.99 & 3.65 & 3.66 \\
\hline \multirow[t]{2}{*}{ 3"' } & 2.56 & 2.56 & 2.55 & 2.43 & 2.64 & 2.41 & 2.42 & 2.65 & 2.41 & 2.68 & 2.61 & 2.55 & 2.26 & 2.28 \\
\hline & 3.18 & 3.18 & 3.19 & 2.91 & 3.19 & 2.91 & 2.85 & 3.20 & 2.85 & 3.25 & 3.13 & 3.14 & 2.83 & 2.60 \\
\hline
\end{tabular}

${ }^{a}$ Calculated using the GIAO method at the mPWIPW91/6-311+G(2d,p) level in MeOH (PCM). ${ }^{b}$ Measured in $C D_{3} \mathrm{OD}(500 \mathrm{MHz})$. ${ }^{c}$ Averaged according to the Boltzmann

distribution theory at $298 \mathrm{~K}$ based on relative Gibbs free energies. ${ }^{d}$ Linearly corrected for the experimental data.

Table S18. Calculated ${ }^{1} \mathrm{H}$ NMR chemical shifts of $\mathbf{1 B}$.

\begin{tabular}{|c|c|c|c|c|c|c|c|c|c|c|c|c|c|c|c|c|c|c|c|c|}
\hline \multirow[b]{2}{*}{ Position } & \multicolumn{19}{|c|}{ calculated $^{a}$} & \multirow{2}{*}{$\begin{array}{c}\text { experimental }^{b} \\
1\end{array}$} \\
\hline & 1B-1 & 1B-2 & 1B-3 & $1 B-4$ & 1B-5 & 1B-6 & 1B-7 & 1B-8 & 1B-9 & 1B-10 & 1B-11 & 1B-12 & 1B-13 & 1B-14 & 1B-15 & 1B-16 & 1B-17 & $\begin{array}{c}\text { averaged- } \\
\mathbf{1 B}^{c}\end{array}$ & $\begin{array}{l}\text { averaged-1B } \\
\text { (corrected) }^{d}\end{array}$ & \\
\hline 1 & 6.23 & 6.22 & 6.24 & 6.24 & 6.23 & 6.23 & 6.15 & 6.24 & 6.16 & 6.13 & 6.22 & 6.01 & 6.14 & 6.21 & 6.03 & 5.95 & 5.96 & 6.20 & 5.78 & 5.69 \\
\hline 2 & 4.03 & 4.02 & 4.04 & 4.03 & 4.02 & 4.02 & 3.73 & 4.03 & 3.73 & 3.73 & 4.02 & 4.10 & 3.74 & 4.01 & 4.12 & 4.02 & 4.02 & 4.00 & 3.61 & 3.66 \\
\hline 3 & 3.95 & 3.95 & 3.95 & 3.95 & 3.95 & 3.95 & 4.14 & 3.95 & 4.14 & 4.14 & 3.95 & 4.29 & 4.14 & 3.95 & 4.27 & 4.27 & 4.27 & 3.99 & 3.61 & 3.77 \\
\hline 4 & 5.08 & 5.07 & 5.09 & 5.08 & 5.08 & 5.07 & 5.53 & 5.11 & 5.53 & 5.52 & 5.06 & 4.76 & 5.53 & 5.06 & 4.79 & 5.45 & 5.45 & 5.13 & 4.72 & 5.00 \\
\hline 5 & 4.35 & 4.35 & 4.36 & 4.35 & 4.36 & 4.35 & 4.18 & 4.36 & 4.18 & 4.15 & 4.34 & 4.23 & 4.15 & 4.34 & 4.24 & 4.18 & 4.18 & 4.32 & 3.93 & 3.98 \\
\hline \multirow[t]{2}{*}{6} & 4.97 & 4.98 & 5.07 & 4.98 & 5.08 & 4.99 & 5.09 & 5.06 & 5.09 & 5.22 & 5.00 & 5.13 & 5.22 & 5.01 & 5.17 & 4.64 & 4.65 & 5.02 & 4.61 & 4.64 \\
\hline & 3.97 & 3.97 & 3.95 & 3.97 & 3.95 & 3.97 & 3.94 & 3.96 & 3.95 & 3.92 & 3.95 & 3.82 & 3.93 & 3.95 & 3.82 & 4.21 & 4.20 & 3.96 & 3.58 & 3.72 \\
\hline 3" & 7.07 & 7.07 & 7.11 & 7.00 & 7.11 & 7.00 & 7.30 & 7.11 & 7.30 & 7.29 & 6.99 & 7.45 & 7.29 & 6.99 & 7.40 & 7.25 & 7.25 & 7.11 & 6.66 & 6.59 \\
\hline 1"' & 4.47 & 4.46 & 4.48 & 4.36 & 4.47 & 4.35 & 4.84 & 4.53 & 4.85 & 4.82 & 4.32 & 4.07 & 4.83 & 4.31 & 4.00 & 4.86 & 4.86 & 4.48 & 4.09 & 4.33 \\
\hline $2^{\prime \prime \prime}$ & 4.15 & 4.14 & 4.45 & 4.09 & 4.45 & 4.08 & 4.78 & 4.46 & 4.78 & 4.34 & 3.81 & 4.96 & 4.34 & 3.81 & 4.45 & 4.10 & 4.10 & 4.27 & 3.88 & 3.66 \\
\hline \multirow[t]{2}{*}{$3^{\prime \prime \prime}$} & 2.89 & 2.89 & 2.72 & 2.83 & 2.71 & 2.83 & 2.63 & 2.73 & 2.62 & 2.92 & 2.88 & 2.70 & 2.92 & 2.88 & 2.99 & 2.95 & 2.95 & 2.82 & 2.46 & 2.28 \\
\hline & 3.22 & 3.22 & 3.23 & 3.25 & 3.22 & 3.24 & 2.91 & 3.21 & 2.91 & 2.83 & 3.11 & 3.06 & 2.82 & 3.11 & 2.92 & 3.28 & 3.28 & 3.17 & 2.80 & 2.60 \\
\hline
\end{tabular}

${ }^{a}$ Calculated using the GIAO method at the mPWIPW91/6-311+G(2d,p) level in MeOH $(P C M) .{ }^{b}$ Measured in $\mathrm{CD}_{3} \mathrm{OD}(500 \mathrm{MHz}) .{ }^{c}$ Averaged according to the Boltzmann distribution theory at $298 \mathrm{~K}$ based on relative Gibbs free energies.

${ }^{d}$ Linearly corrected for the experimental data. 
Table S19. Calculated ${ }^{1} \mathrm{H}$ NMR chemical shifts of $\mathbf{1 C}$.

\begin{tabular}{|c|c|c|c|c|c|c|c|c|c|c|c|}
\hline \multirow[b]{2}{*}{ Position } & \multicolumn{10}{|c|}{ calculated $^{a}$} & \multirow{2}{*}{$\begin{array}{c}\text { experimental }^{b} \\
1\end{array}$} \\
\hline & 1C-1 & 1C-2 & $1 C-3$ & 1C-4 & 1C-5 & 1C-6 & 1C-7 & 1C-8 & $\begin{array}{c}\text { averaged- } \\
\mathbf{1 C}^{c}\end{array}$ & $\begin{array}{l}\text { averaged-1C } \\
\text { (corrected) }^{d}\end{array}$ & \\
\hline 1 & 6.23 & 6.21 & 6.21 & 6.21 & 6.02 & 6.23 & 6.22 & 6.22 & 6.21 & 5.55 & 5.69 \\
\hline 2 & 4.04 & 4.03 & 4.04 & 4.03 & 4.07 & 4.04 & 4.04 & 4.03 & 4.04 & 3.67 & 3.66 \\
\hline 3 & 4.01 & 4.02 & 4.00 & 4.01 & 4.10 & 4.00 & 3.99 & 4.01 & 4.02 & 3.65 & 3.77 \\
\hline 4 & 5.07 & 5.07 & 5.09 & 5.09 & 5.15 & 5.07 & 5.09 & 5.06 & 5.08 & 4.57 & 5.00 \\
\hline 5 & 4.28 & 4.28 & 4.27 & 4.28 & 4.23 & 4.27 & 4.27 & 4.28 & 4.28 & 3.88 & 3.98 \\
\hline \multirow[t]{2}{*}{6} & 5.23 & 5.22 & 5.26 & 5.25 & 5.18 & 5.23 & 5.26 & 5.22 & 5.23 & 4.70 & 4.64 \\
\hline & 4.00 & 4.00 & 3.99 & 3.99 & 3.99 & 4.00 & 3.99 & 4.00 & 3.99 & 3.63 & 3.72 \\
\hline $2^{\prime}, 6^{\prime}$ & 7.82 & 7.81 & 7.82 & 7.81 & 7.82 & 7.82 & 7.82 & 7.81 & 7.82 & 6.95 & 7.13 \\
\hline 3" & 8.14 & 8.13 & 8.13 & 8.13 & 8.14 & 8.11 & 8.10 & 8.10 & 8.13 & 7.22 & 6.59 \\
\hline 1"' & 4.66 & 4.66 & 4.68 & 4.67 & 4.73 & 4.57 & 4.61 & 4.58 & 4.66 & 4.21 & 4.33 \\
\hline 2"' & 4.32 & 4.33 & 4.31 & 4.31 & 4.35 & 4.30 & 4.30 & 4.31 & 4.32 & 3.91 & 3.66 \\
\hline \multirow[t]{2}{*}{ 3"' } & 2.66 & 2.66 & 2.58 & 2.58 & 2.65 & 2.79 & 2.72 & 2.78 & 2.65 & 2.46 & 2.28 \\
\hline & 2.84 & 2.85 & 2.88 & 2.88 & 2.86 & 2.86 & 2.91 & 2.87 & 2.85 & 2.64 & 2.60 \\
\hline
\end{tabular}

${ }^{a}$ Calculated using the GIAO method at the mPWIPW91/6-311+G(2d,p) level in MeOH $(\mathrm{PCM}) .{ }^{b}$ Measured in $\mathrm{CD}{ }_{3} \mathrm{OD}(500 \mathrm{MHz}) .{ }^{c}$ Averaged

according to the Boltzmann distribution theory at $298 \mathrm{~K}$ based on relative Gibbs free energies. ${ }^{d}$ Linearly corrected for the experimental data.

Table S20. Calculated ${ }^{1} \mathrm{H}$ NMR chemical shifts of 1D.

\begin{tabular}{|c|c|c|c|c|c|c|c|c|c|c|c|c|}
\hline \multirow[b]{2}{*}{ Position } & \multicolumn{11}{|c|}{ calculated $^{a}$} & \multirow{2}{*}{$\begin{array}{c}\text { experimental } \\
1\end{array}$} \\
\hline & 1D-1 & 1D-2 & 1D-3 & 1D-4 & 1D-5 & 1D-6 & 1D-7 & 1D-8 & 1D-9 & $\begin{array}{c}\text { averaged- } \\
\mathbf{1 A}^{c}\end{array}$ & $\begin{array}{r}\text { averaged-1D } \\
{\text { (corrected) }{ }^{d}}^{2}\end{array}$ & \\
\hline 1 & 6.18 & 6.17 & 6.17 & 6.17 & 6.17 & 6.18 & 6.17 & 6.17 & 6.17 & 6.17 & 5.53 & 5.69 \\
\hline 2 & 4.01 & 4.03 & 4.02 & 4.03 & 4.02 & 4.02 & 4.03 & 4.03 & 4.04 & 4.02 & 3.62 & 3.66 \\
\hline 3 & 3.95 & 3.95 & 3.94 & 3.94 & 3.95 & 3.96 & 3.95 & 3.96 & 3.96 & 3.95 & 3.55 & 3.77 \\
\hline 4 & 4.99 & 4.99 & 4.95 & 4.95 & 4.96 & 4.95 & 4.97 & 4.95 & 4.96 & 4.98 & 4.47 & 5.00 \\
\hline 5 & 4.24 & 4.25 & 4.24 & 4.24 & 4.24 & 4.26 & 4.25 & 4.26 & 4.27 & 4.24 & 3.82 & 3.98 \\
\hline \multirow[t]{2}{*}{6} & 5.13 & 5.14 & 5.15 & 5.16 & 5.15 & 5.12 & 5.16 & 5.13 & 5.13 & 5.14 & 4.61 & 4.64 \\
\hline & 3.97 & 3.98 & 4.00 & 4.01 & 3.99 & 3.99 & 4.00 & 3.99 & 3.99 & 3.98 & 3.58 & 3.72 \\
\hline $2^{\prime}, 6^{\prime}$ & 7.81 & 7.82 & 7.81 & 7.81 & 7.80 & 7.81 & 7.81 & 7.81 & 7.81 & 7.81 & 6.99 & 7.13 \\
\hline 3" & 8.19 & 8.19 & 8.08 & 8.09 & 8.10 & 8.08 & 8.10 & 8.09 & 8.10 & 8.16 & 7.29 & 6.59 \\
\hline $1^{\prime \prime \prime}$ & 4.73 & 4.73 & 4.56 & 4.56 & 4.54 & 4.70 & 4.55 & 4.70 & 4.68 & 4.68 & 4.21 & 4.33 \\
\hline $2^{\prime \prime \prime}$ & 4.88 & 4.85 & 4.36 & 4.36 & 4.40 & 5.01 & 4.40 & 5.00 & 4.79 & 4.74 & 4.26 & 3.66 \\
\hline \multirow[t]{2}{*}{ 3"' } & 2.67 & 2.69 & 2.89 & 2.88 & 2.83 & 2.60 & 2.83 & 2.60 & 2.52 & 2.73 & 2.47 & 2.28 \\
\hline & 2.99 & 3.00 & 2.81 & 2.80 & 2.97 & 2.95 & 2.96 & 2.95 & 2.77 & 2.95 & 2.66 & 2.60 \\
\hline
\end{tabular}

${ }^{a}$ Calculated using the GIAO method at the mPWIPW91/6-311+G(2d,p) level in MeOH (PCM). ${ }^{b}$ Measured in $\mathrm{CD}_{3} \mathrm{OD}(500 \mathrm{MHz}) .{ }^{c}$ Averaged according

to the Boltzmann distribution theory at $298 \mathrm{~K}$ based on relative Gibbs free energies. ${ }^{d}$ Linearly corrected for the experimental data. 
Table S21. Calculated ${ }^{1} \mathrm{H}$ NMR chemical shifts of $\mathbf{1 E}$.

\begin{tabular}{|c|c|c|c|c|c|c|c|c|c|c|c|c|c|c|c|c|c|}
\hline \multirow[b]{2}{*}{ Position } & \multicolumn{16}{|c|}{ calculated $^{a}$} & \multirow{2}{*}{$\begin{array}{c}\text { experimental }^{b} \\
1\end{array}$} \\
\hline & 1E-1 & $1 \mathrm{E}-2$ & $1 \mathrm{E}-3$ & $1 \mathrm{E}-4$ & 1E-5 & 1E-6 & 1E-7 & 1E-8 & 1E-9 & 1E-10 & 1E-11 & 1E-12 & 1E-13 & 1E-14 & $\begin{array}{c}\text { averaged- } \\
\mathbf{1 A}^{c}\end{array}$ & $\begin{array}{l}\text { averaged-1E } \\
{\text { (corrected) }{ }^{d}}^{\text {col }}\end{array}$ & \\
\hline 1 & 6.07 & 6.13 & 6.12 & 6.16 & 6.08 & 6.16 & 6.07 & 6.08 & 6.16 & 6.16 & 6.21 & 6.13 & 6.16 & 6.16 & 6.12 & 5.68 & 5.69 \\
\hline 2 & 4.20 & 4.20 & 4.19 & 4.19 & 4.18 & 4.20 & 4.19 & 4.18 & 4.19 & 4.20 & 4.12 & 4.15 & 4.21 & 4.19 & 4.19 & 3.84 & 3.66 \\
\hline 3 & 4.18 & 4.34 & 4.33 & 4.31 & 4.18 & 4.31 & 4.18 & 4.17 & 4.33 & 4.33 & 4.38 & 4.37 & 4.35 & 4.34 & 4.27 & 3.92 & 3.77 \\
\hline 4 & 5.15 & 4.79 & 4.79 & 4.79 & 5.14 & 4.80 & 5.14 & 5.14 & 4.80 & 4.81 & 4.72 & 4.74 & 4.79 & 4.78 & 4.92 & 4.53 & 5.00 \\
\hline 5 & 4.11 & 4.39 & 4.40 & 4.35 & 4.12 & 4.35 & 4.11 & 4.11 & 4.36 & 4.35 & 4.35 & 4.38 & 4.37 & 4.37 & 4.28 & 3.93 & 3.98 \\
\hline \multirow[t]{2}{*}{6} & 5.26 & 4.46 & 4.47 & 4.47 & 5.26 & 4.47 & 5.24 & 5.24 & 4.46 & 4.46 & 4.38 & 4.43 & 4.45 & 4.45 & 4.75 & 4.37 & 4.64 \\
\hline & 4.17 & 5.24 & 5.23 & 4.66 & 4.17 & 4.66 & 4.17 & 4.16 & 4.65 & 4.66 & 5.19 & 5.20 & 4.76 & 4.76 & 4.67 & 4.30 & 3.72 \\
\hline $2^{\prime}, 6^{\prime}$ & 7.78 & 7.78 & 7.82 & 7.81 & 7.79 & 7.77 & 7.78 & 7.79 & 7.82 & 7.78 & 7.81 & 7.82 & 7.78 & 7.82 & 7.79 & 7.27 & 7.13 \\
\hline 3" & 7.41 & 7.46 & 7.46 & 7.48 & 7.40 & 7.47 & 7.42 & 7.41 & 7.51 & 7.50 & 7.53 & 7.52 & 7.51 & 7.51 & 7.45 & 6.95 & 6.59 \\
\hline 1"' & 4.75 & 3.95 & 3.94 & 4.16 & 4.74 & 4.17 & 4.73 & 4.72 & 4.22 & 4.23 & 4.05 & 4.06 & 4.13 & 4.12 & 4.31 & 3.95 & 4.33 \\
\hline 2"' & 3.42 & 3.45 & 3.46 & 3.41 & 3.42 & 3.41 & 3.40 & 3.40 & 3.43 & 3.43 & 3.45 & 3.46 & 3.46 & 3.46 & 3.43 & 3.11 & 3.66 \\
\hline \multirow[t]{2}{*}{ 3"' } & 2.81 & 2.79 & 2.79 & 2.62 & 2.81 & 2.62 & 2.82 & 2.83 & 2.70 & 2.69 & 2.84 & 2.85 & 2.69 & 2.69 & 2.75 & 2.47 & 2.28 \\
\hline & 2.91 & 3.07 & 3.06 & 3.07 & 2.91 & 3.07 & 3.00 & 3.00 & 3.18 & 3.18 & 3.13 & 3.13 & 3.31 & 3.31 & 3.03 & 2.74 & 2.60 \\
\hline
\end{tabular}

${ }^{a}$ Calculated using the GIAO method at the mPWIPW91/6-311+G(2d,p) level in MeOH $(\mathrm{PCM}) .{ }^{b}$ Measured in $\mathrm{CD}_{3} \mathrm{OD}(500 \mathrm{MHz}) .{ }^{c}$ Averaged according to the Boltzmann distribution theory at $298 \mathrm{~K}$ based

on relative Gibbs free energies. ${ }^{d}$ Linearly corrected for the experimental data.

Table S22. Calculated ${ }^{1} \mathrm{H}$ NMR chemical shifts of $\mathbf{1 F}$.

\begin{tabular}{|c|c|c|c|c|c|c|c|c|c|c|c|c|c|c|c|c|c|c|c|}
\hline \multirow[b]{2}{*}{ Position } & \multicolumn{18}{|c|}{ calculated $^{a}$} & \multirow{2}{*}{$\begin{array}{c}\text { experimental }^{b} \\
1\end{array}$} \\
\hline & $1 F-1$ & $1 F-2$ & $1 F-3$ & $1 F-4$ & $1 F-5$ & $1 F-6$ & 1F-7 & 1F-8 & 1F-9 & $1 F-10$ & 1F-11 & 1F-12 & 1F-13 & 1F-14 & 1F-15 & 1F-16 & $\begin{array}{c}\text { averaged- } \\
\mathbf{1 A}^{c}\end{array}$ & $\begin{array}{c}\text { averaged-1F } \\
\text { (corrected) }^{d}\end{array}$ & \\
\hline 1 & 6.06 & 6.07 & 6.05 & 6.06 & 6.06 & 6.07 & 6.06 & 6.07 & 6.05 & 6.06 & 6.05 & 6.07 & 6.06 & 6.16 & 6.05 & 5.94 & 6.06 & 5.61 & 5.69 \\
\hline 2 & 4.12 & 4.13 & 4.11 & 4.13 & 4.12 & 4.13 & 4.12 & 4.13 & 4.11 & 4.12 & 4.11 & 4.13 & 4.12 & 4.15 & 4.11 & 4.10 & 4.12 & 3.76 & 3.66 \\
\hline 3 & 4.13 & 4.14 & 4.12 & 4.12 & 4.13 & 4.13 & 4.13 & 4.14 & 4.12 & 4.12 & 4.13 & 4.13 & 4.12 & 4.29 & 4.12 & 4.04 & 4.13 & 3.77 & 3.77 \\
\hline 4 & 5.02 & 5.03 & 5.03 & 5.04 & 5.01 & 5.01 & 5.03 & 5.03 & 4.99 & 5.00 & 5.03 & 5.04 & 5.02 & 4.77 & 5.01 & 5.58 & 5.03 & 4.63 & 5.00 \\
\hline 5 & 4.09 & 4.09 & 4.07 & 4.07 & 4.10 & 4.10 & 4.09 & 4.09 & 4.08 & 4.07 & 4.08 & 4.07 & 4.09 & 4.33 & 4.08 & 4.05 & 4.08 & 3.73 & 3.98 \\
\hline \multirow[t]{2}{*}{6} & 5.31 & 5.31 & 5.28 & 5.28 & 5.34 & 5.34 & 5.30 & 5.30 & 5.30 & 5.29 & 5.29 & 5.28 & 5.32 & 4.37 & 5.28 & 5.30 & 5.29 & 4.88 & 4.64 \\
\hline & 4.05 & 4.05 & 4.04 & 4.05 & 4.06 & 4.07 & 4.05 & 4.06 & 4.01 & 4.02 & 4.04 & 4.04 & 4.07 & 4.66 & 4.03 & 4.04 & 4.06 & 3.70 & 3.72 \\
\hline $2^{\prime}, 6^{\prime}$ & 7.79 & 7.77 & 7.79 & 7.77 & 7.79 & 7.77 & 7.79 & 7.77 & 7.79 & 7.77 & 7.79 & 7.77 & 7.79 & 7.79 & 7.79 & 7.78 & 7.78 & 7.26 & 7.13 \\
\hline 3" & 7.45 & 7.45 & 7.42 & 7.42 & 7.34 & 7.34 & 7.40 & 7.41 & 7.33 & 7.33 & 7.45 & 7.45 & 7.32 & 7.53 & 7.31 & 7.04 & 7.41 & 6.90 & 6.59 \\
\hline 1"' & 4.70 & 4.70 & 4.62 & 4.63 & 4.52 & 4.52 & 4.67 & 4.68 & 4.58 & 4.58 & 4.62 & 4.63 & 4.50 & 4.18 & 4.59 & 4.44 & 4.63 & 4.24 & 4.33 \\
\hline $2^{\prime \prime \prime}$ & 3.41 & 3.40 & 3.38 & 3.37 & 3.51 & 3.51 & 3.41 & 3.40 & 3.43 & 3.43 & 3.32 & 3.31 & 3.43 & 3.42 & 3.35 & 3.49 & 3.41 & 3.08 & 3.66 \\
\hline \multirow[t]{2}{*}{$3^{\prime \prime \prime}$} & 2.92 & 2.92 & 2.97 & 2.97 & 2.95 & 2.95 & 2.99 & 2.99 & 2.88 & 2.87 & 2.95 & 2.95 & 2.97 & 2.71 & 2.95 & 2.88 & 2.94 & 2.64 & 2.28 \\
\hline & 3.26 & 3.26 & 3.12 & 3.11 & 3.21 & 3.21 & 3.22 & 3.22 & 3.10 & 3.10 & 3.08 & 3.08 & 3.12 & 2.92 & 2.91 & 3.26 & 3.18 & 2.87 & 2.60 \\
\hline
\end{tabular}

${ }^{a}$ Calculated using the GIAO method at the mPWIPW91/6-311+G(2d,p) level in MeOH (PCM). ${ }^{b}$ Measured in $C D_{3} \mathrm{OD}(500 \mathrm{MHz}) .{ }^{c}$ Averaged according to the Boltzmann distribution theory at $298 \mathrm{~K}$ based on relative Gibbs free energies. ${ }^{d}$ Linearly corrected for the experimental data. 
Table S23. Calculated ${ }^{1} \mathrm{H}$ NMR chemical shifts of $\mathbf{1 G}$.

\begin{tabular}{|c|c|c|c|c|c|c|c|c|c|c|c|c|}
\hline \multirow[b]{2}{*}{ Position } & \multicolumn{11}{|c|}{ calculated $^{a}$} & \multirow{2}{*}{$\frac{\text { experimental }^{b}}{1}$} \\
\hline & 1G-1 & $1 \mathrm{G}-2$ & $1 \mathrm{G}-3$ & $1 \mathrm{G}-4$ & 1G-5 & 1G-6 & 1G-7 & 1G-8 & 1G-9 & $\begin{array}{c}\text { averaged- } \\
\mathbf{1 A}^{c}\end{array}$ & $\begin{array}{c}\text { averaged-1G } \\
\text { (corrected) }^{d}\end{array}$ & \\
\hline 1 & 6.11 & 6.12 & 6.09 & 6.10 & 6.11 & 6.12 & 6.05 & 6.09 & 6.11 & 6.11 & 5.46 & $\begin{array}{l}5.69 \\
\end{array}$ \\
\hline 2 & 4.15 & 4.17 & 4.15 & 4.15 & 4.16 & 4.17 & 4.13 & 4.15 & 4.16 & 4.16 & 3.76 & 3.66 \\
\hline 3 & 4.20 & 4.20 & 4.18 & 4.21 & 4.18 & 4.22 & 4.20 & 4.20 & 4.20 & 4.20 & 3.79 & 3.77 \\
\hline 4 & 5.16 & 5.16 & 5.14 & 5.17 & 5.14 & 5.17 & 5.75 & 5.14 & 5.14 & 5.17 & 4.64 & 5.00 \\
\hline 5 & 4.43 & 4.43 & 4.40 & 4.43 & 4.40 & 4.43 & 4.36 & 4.40 & 4.40 & 4.43 & 3.99 & 3.98 \\
\hline \multirow[t]{2}{*}{6} & 5.48 & 5.48 & 5.48 & 5.46 & 5.48 & 5.46 & 5.42 & 5.47 & 5.47 & 5.48 & 4.91 & 4.64 \\
\hline & 3.87 & 3.87 & 3.87 & 3.86 & 3.88 & 3.87 & 3.88 & 3.86 & 3.87 & 3.87 & 3.50 & 3.72 \\
\hline $2^{\prime}, 6^{\prime}$ & 7.82 & 7.81 & 7.82 & 7.82 & 7.81 & 7.80 & 7.78 & 7.81 & 7.80 & 7.81 & 6.95 & 7.13 \\
\hline 3" & 8.07 & 8.07 & 8.04 & 8.08 & 8.04 & 8.08 & 7.79 & 8.04 & 8.04 & 8.06 & 7.17 & 6.59 \\
\hline $1^{\prime \prime \prime}$ & 5.07 & 5.07 & 4.94 & 5.08 & 4.94 & 5.09 & 5.26 & 4.96 & 4.96 & 5.05 & 4.54 & 4.33 \\
\hline $2 " '$ & 3.30 & 3.31 & 3.31 & 3.31 & 3.32 & 3.32 & 3.21 & 3.32 & 3.33 & 3.30 & 3.01 & 3.66 \\
\hline \multirow[t]{2}{*}{$3^{\prime \prime \prime}$} & 2.61 & 2.61 & 2.65 & 2.67 & 2.65 & 2.67 & 2.59 & 2.69 & 2.69 & 2.63 & 2.42 & 2.28 \\
\hline & 3.16 & 3.15 & 3.32 & 3.19 & 3.31 & 3.18 & 3.14 & 3.37 & 3.36 & 3.19 & 2.91 & 2.60 \\
\hline
\end{tabular}

${ }^{a}$ Calculated using the GIAO method at the mPWIPW91/6-311+G(2d,p) level in $\mathrm{MeOH}(\mathrm{PCM}) .{ }^{b}$ Measured in $\mathrm{CD}{ }_{3} \mathrm{OD}(500 \mathrm{MHz}) .{ }^{c}$ Averaged according

to the Boltzmann distribution theory at $298 \mathrm{~K}$ based on relative Gibbs free energies. ${ }^{d}$ Linearly corrected for the experimental data.

Table S24. Calculated ${ }^{1} \mathrm{H}$ NMR chemical shifts of $\mathbf{1 H}$.

\begin{tabular}{|c|c|c|c|c|c|c|c|c|c|c|c|c|c|c|c|c|c|c|c|c|c|}
\hline \multirow[b]{2}{*}{ Position } & \multicolumn{20}{|c|}{ calculated $^{a}$} & \multirow{2}{*}{$\begin{array}{c}\text { experimental }^{b} \\
1\end{array}$} \\
\hline & $1 \mathrm{H}-1$ & $1 \mathrm{H}-2$ & $1 \mathrm{H}-3$ & $1 \mathrm{H}-4$ & $1 \mathrm{H}-5$ & $1 \mathrm{H}-6$ & 1H-7 & 1H-8 & 1H-9 & $1 \mathrm{H}-10$ & $1 \mathrm{H}-11$ & $1 \mathrm{H}-12$ & $1 \mathrm{H}-13$ & $1 \mathrm{H}-14$ & $1 \mathrm{H}-15$ & $1 \mathrm{H}-16$ & $1 \mathrm{H}-17$ & 1H-18 & $\begin{array}{c}\text { averaged- } \\
\mathbf{1 A}^{c}\end{array}$ & $\begin{array}{c}\text { averaged-1H } \\
\text { (corrected) }^{d}\end{array}$ & \\
\hline 1 & 6.09 & 6.08 & 6.09 & 6.14 & 6.08 & 6.09 & 6.08 & 6.12 & 6.09 & 6.15 & 6.07 & 6.13 & 6.07 & 6.12 & 6.09 & 6.15 & 6.15 & 6.14 & 6.10 & 5.44 & 5.69 \\
\hline 2 & 4.16 & 4.15 & 4.14 & 4.16 & 4.13 & 4.15 & 4.14 & 4.17 & 4.16 & 4.16 & 4.14 & 4.15 & 4.13 & 4.16 & 4.14 & 4.17 & 4.15 & 4.16 & 4.15 & 3.70 & 3.66 \\
\hline 3 & 4.14 & 4.11 & 4.13 & 4.20 & 4.11 & 4.14 & 4.13 & 4.17 & 4.14 & 4.20 & 4.14 & 4.20 & 4.14 & 4.17 & 4.14 & 4.22 & 4.19 & 4.22 & 4.14 & 3.70 & 3.77 \\
\hline 4 & 5.16 & 5.17 & 5.15 & 5.13 & 5.15 & 5.18 & 5.18 & 5.18 & 5.17 & 5.15 & 5.06 & 5.15 & 5.06 & 5.18 & 5.17 & 5.16 & 5.07 & 5.16 & 5.15 & 4.59 & 5.00 \\
\hline 5 & 4.34 & 4.35 & 4.34 & 4.51 & 4.35 & 4.36 & 4.37 & 4.52 & 4.43 & 4.55 & 4.31 & 4.55 & 4.31 & 4.52 & 4.43 & 4.58 & 4.54 & 4.58 & 4.40 & 3.92 & 3.98 \\
\hline \multirow[t]{2}{*}{6} & 5.46 & 5.51 & 5.47 & 5.45 & 5.51 & 5.44 & 5.44 & 5.53 & 5.50 & 5.45 & 5.41 & 5.45 & 5.41 & 5.53 & 5.50 & 5.46 & 5.34 & 5.46 & 5.47 & 4.88 & 4.64 \\
\hline & 3.89 & 3.90 & 3.88 & 3.88 & 3.90 & 3.90 & 3.89 & 3.93 & 3.92 & 3.89 & 3.84 & 3.88 & 3.83 & 3.92 & 3.91 & 3.89 & 4.16 & 3.89 & 3.89 & 3.47 & 3.72 \\
\hline $2^{\prime}, 6^{\prime}$ & 7.80 & 7.79 & 7.82 & 7.80 & 7.81 & 7.80 & 7.81 & 7.81 & 7.80 & 7.81 & 7.80 & 7.82 & 7.81 & 7.83 & 7.82 & 7.81 & 7.79 & 7.83 & 7.81 & 6.96 & 7.13 \\
\hline $3^{\prime \prime}$ & 8.14 & 8.03 & 8.14 & 8.10 & 8.03 & 8.12 & 8.12 & 8.15 & 8.04 & 8.09 & 8.05 & 8.09 & 8.05 & 8.15 & 8.03 & 8.09 & 7.99 & 8.10 & 8.09 & 7.21 & 6.59 \\
\hline $1^{\prime \prime \prime}$ & 5.30 & 5.17 & 5.30 & 5.44 & 5.17 & 5.33 & 5.33 & 5.48 & 5.32 & 5.44 & 5.05 & 5.45 & 5.06 & 5.49 & 5.32 & 5.51 & 4.84 & 5.52 & 5.29 & 4.72 & 4.33 \\
\hline $2^{\prime \prime \prime}$ & 3.45 & 3.49 & 3.45 & 3.33 & 3.49 & 3.41 & 3.41 & 3.29 & 3.27 & 3.30 & 3.33 & 3.30 & 3.33 & 3.29 & 3.27 & 3.28 & 3.50 & 3.28 & 3.41 & 3.04 & 3.66 \\
\hline \multirow[t]{2}{*}{$3^{\prime \prime \prime}$} & 2.68 & 2.71 & 2.69 & 2.70 & 2.71 & 2.74 & 2.74 & 2.73 & 2.68 & 2.77 & 2.69 & 2.77 & 2.69 & 2.73 & 2.68 & 2.80 & 2.94 & 2.80 & 2.71 & 2.42 & 2.28 \\
\hline & 3.69 & 3.47 & 3.70 & 2.96 & 3.48 & 3.49 & 3.49 & 2.72 & 2.82 & 2.97 & 3.61 & 2.98 & 3.61 & 2.72 & 2.83 & 2.93 & 3.09 & 2.94 & 3.38 & 3.01 & 2.60 \\
\hline
\end{tabular}

${ }^{a}$ Calculated using the GIAO method at the $\mathrm{mPWI} P \mathrm{P} 91 / 6-311+G(2 d, p)$ level in MeOH $(P C M) .{ }^{b}$ Measured in $\mathrm{CD}{ }_{3} \mathrm{OD}(500 \mathrm{MHz}) .{ }^{c}$ Averaged according to the Boltzmann distribution theory at $298 \mathrm{~K}$ based on relative Gibbs free energies.

${ }^{d}$ Linearly corrected for the experimental data. 
Table S25. Calculated ${ }^{1} \mathrm{H}$ NMR chemical shifts of $\mathbf{1}^{\prime} \mathbf{A}$.

\begin{tabular}{|c|c|c|c|c|c|c|c|c|c|}
\hline \multirow[b]{2}{*}{ Position } & \multicolumn{8}{|c|}{ calculated $^{a}$} & \multirow{2}{*}{$\begin{array}{c}\text { experimental }^{b} \\
1\end{array}$} \\
\hline & 1'A-1 & 1'A-2 & 1'A-3 & $1^{\prime} \mathrm{A}-4$ & $1^{\prime} \mathrm{A}-5$ & 1'A-6 & $\begin{array}{c}\text { averaged- } \\
\mathbf{1}^{\prime} \mathbf{A}^{c}\end{array}$ & $\begin{array}{c}\text { averaged-1'A } \\
{\text { (corrected) }{ }^{d}}^{d}\end{array}$ & \\
\hline 1 & 6.04 & 6.13 & 6.11 & 6.07 & 6.06 & 6.09 & 6.08 & 5.54 & 5.69 \\
\hline 2 & 4.17 & 4.06 & 4.03 & 4.10 & 4.10 & 4.04 & 4.11 & 3.72 & 3.66 \\
\hline 3 & 4.38 & 4.00 & 4.00 & 4.42 & 4.43 & 4.07 & 4.24 & 3.84 & 3.77 \\
\hline 4 & 5.87 & 5.70 & 5.69 & 5.89 & 5.89 & 5.73 & 5.81 & 5.29 & 5.00 \\
\hline 5 & 4.22 & 4.23 & 4.23 & 4.25 & 4.25 & 4.22 & 4.23 & 3.83 & 3.98 \\
\hline \multirow[t]{2}{*}{6} & 4.83 & 4.88 & 4.88 & 4.86 & 4.85 & 4.89 & 4.85 & 4.41 & 4.64 \\
\hline & 4.17 & 4.15 & 4.14 & 4.30 & 4.29 & 4.29 & 4.18 & 3.79 & 3.72 \\
\hline $2^{\prime}, 6^{\prime}$ & 7.71 & 7.73 & 7.75 & 7.71 & 7.72 & 7.73 & 7.72 & 7.07 & 7.13 \\
\hline 3" & 7.20 & 7.18 & 7.18 & 7.21 & 7.21 & 7.20 & 7.19 & 6.58 & 6.59 \\
\hline 1"' & 5.05 & 5.01 & 5.02 & 5.10 & 5.10 & 5.09 & 5.05 & 4.59 & 4.33 \\
\hline $2^{\prime \prime \prime}$ & 4.09 & 4.06 & 4.05 & 4.13 & 4.13 & 4.11 & 4.09 & 3.70 & 3.66 \\
\hline \multirow[t]{2}{*}{$3^{\prime \prime \prime}$} & 2.43 & 2.42 & 2.42 & 2.50 & 2.49 & 2.49 & 2.44 & 2.18 & 2.28 \\
\hline & 2.78 & 2.79 & 2.79 & 2.83 & 2.83 & 2.85 & 2.79 & 2.50 & 2.60 \\
\hline
\end{tabular}

${ }^{a}$ Calculated using the GIAO method at the mPWIPW91/6-311+G(2d,p) level in MeOH $(P C M) .{ }^{b}$ Measured in $C D{ }_{3} O D$

$(500 \mathrm{MHz}) .{ }^{c}$ Averaged according to the Boltzmann distribution theory at $298 \mathrm{~K}$ based on relative Gibbs free energies.

${ }^{d}$ Linearly corrected for the experimental data.

Table S26. Calculated ${ }^{1} \mathrm{H}$ NMR chemical shifts of $\mathbf{1}^{\prime} \mathbf{B}$.

\begin{tabular}{|c|c|c|c|c|c|c|c|c|c|c|c|c|c|c|c|}
\hline \multirow[b]{2}{*}{ Position } & \multicolumn{14}{|c|}{ calculated $^{a}$} & \multirow{2}{*}{$\frac{\text { experimental }^{b}}{1}$} \\
\hline & 1'B-1 & $1^{\prime} \mathbf{B}-2$ & 1'B-3 & 1'B-4 & I'B-5 & 1'B-6 & 1'B-7 & 1'B-8 & 1'B-9 & 1'B-10 & 1'B-11 & $1^{\prime} \mathrm{B}-12$ & $\begin{array}{c}\text { averaged- } \\
\mathbf{1}^{\prime} \mathbf{B}^{c}\end{array}$ & $\begin{array}{l}\text { averaged-1'B } \mathbf{B} \\
\text { (corrected) }^{d}\end{array}$ & \\
\hline 1 & 6.12 & 6.08 & 6.07 & 6.07 & 6.16 & 6.18 & 6.16 & 6.15 & 6.08 & 6.30 & 6.16 & 6.23 & 6.12 & 5.56 & 5.69 \\
\hline 2 & 4.08 & 4.10 & 4.10 & 3.94 & 3.92 & 3.93 & 3.91 & 4.01 & 3.91 & 4.04 & 4.03 & 4.01 & 4.04 & 3.62 & 3.66 \\
\hline 3 & 4.01 & 4.42 & 4.42 & 5.44 & 5.49 & 5.49 & 5.49 & 5.32 & 5.41 & 5.47 & 5.33 & 5.41 & 4.65 & 4.19 & 3.77 \\
\hline 4 & 5.57 & 5.75 & 5.75 & 4.60 & 4.54 & 4.51 & 4.54 & 4.52 & 4.60 & 4.48 & 4.52 & 4.51 & 5.25 & 4.76 & 5.00 \\
\hline 5 & 4.23 & 4.23 & 4.23 & 5.33 & 5.43 & 5.49 & 5.43 & 5.33 & 5.32 & 5.52 & 5.33 & 5.41 & 4.65 & 4.20 & 3.98 \\
\hline \multirow[t]{2}{*}{6} & 4.67 & 4.65 & 4.64 & 5.63 & 5.40 & 5.35 & 5.41 & 5.68 & 5.68 & 5.37 & 5.68 & 5.49 & 4.97 & 4.49 & 4.64 \\
\hline & 4.18 & 4.31 & 4.30 & 3.84 & 3.85 & 3.86 & 3.86 & 3.83 & 3.84 & 3.85 & 3.83 & 3.85 & 4.10 & 3.68 & 3.72 \\
\hline $2^{\prime}, 6^{\prime}$ & 7.71 & 7.70 & 7.72 & 7.82 & 7.82 & 7.81 & 7.78 & 7.79 & 7.77 & 7.83 & 7.83 & 7.79 & 7.75 & 7.07 & 7.13 \\
\hline $3^{\prime \prime}$ & 7.23 & 7.26 & 7.26 & 7.73 & 7.73 & 7.69 & 7.73 & 7.72 & 7.73 & 7.68 & 7.72 & 7.73 & 7.42 & 6.77 & 6.59 \\
\hline 1"' & 5.01 & 5.09 & 5.09 & 4.59 & 4.60 & 4.59 & 4.60 & 4.58 & 4.60 & 4.59 & 4.59 & 4.59 & 4.88 & 4.41 & 4.33 \\
\hline $2^{\prime \prime \prime}$ & 4.09 & 4.20 & 4.19 & 4.03 & 3.89 & 3.75 & 3.88 & 4.03 & 4.03 & 3.76 & 4.03 & 3.91 & 4.06 & 3.65 & 3.66 \\
\hline \multirow[t]{2}{*}{ 3"' } & 2.84 & 2.90 & 2.89 & 2.55 & 2.53 & 2.66 & 2.52 & 2.56 & 2.54 & 2.65 & 2.57 & 2.50 & 2.76 & 2.43 & 2.60 \\
\hline & 2.68 & 2.72 & 2.72 & 2.25 & 2.23 & 2.25 & 2.23 & 2.25 & 2.24 & 2.25 & 2.26 & 2.25 & 2.53 & 2.22 & 2.28 \\
\hline
\end{tabular}

${ }^{a}$ Calculated using the GIAO method at the mPWIPW91/6-311+G(2d,p) level in $\mathrm{MeOH}(\mathrm{PCM}) .{ }^{b}$ Measured in $\mathrm{CD}_{3} \mathrm{OD}(500 \mathrm{MHz}) .{ }^{c}$ Averaged according to the Boltzmann distribution theory at $298 \mathrm{~K}$ based on relative Gibbs free energies. ${ }^{d}$ Linearly corrected for the experimental data. 
Table S27. Calculated ${ }^{1} \mathrm{H}$ NMR chemical shifts of $\mathbf{1}^{\prime} \mathbf{C}$.

\begin{tabular}{|c|c|c|c|c|c|c|c|c|c|c|c|c|c|c|c|c|c|c|c|}
\hline \multirow[b]{2}{*}{ Position } & \multicolumn{18}{|c|}{ calculated $^{a}$} & \multirow{2}{*}{$\begin{array}{c}\text { experimental }^{b} \\
1\end{array}$} \\
\hline & $1^{\prime} \mathrm{C}-1$ & $1^{\prime} \mathrm{C}-2$ & $1^{\prime} \mathrm{C}-3$ & $1^{\prime} \mathrm{C}-4$ & $1^{\prime} \mathrm{C}-5$ & $1^{\prime} \mathrm{C}-6$ & $1^{\prime} \mathrm{C}-7$ & $1 ' \mathrm{C}-8$ & $1^{\prime} \mathrm{C}-9$ & $1^{\prime} \mathrm{C}-10$ & $1^{\prime} \mathrm{C}-11$ & $1^{\prime} \mathrm{C}-12$ & $1^{\prime} \mathrm{C}-13$ & $1^{\prime} \mathrm{C}-14$ & $1^{\prime} \mathrm{C}-15$ & $1 ' \mathrm{C}-16$ & $\begin{array}{c}\text { averaged- } \\
\mathbf{1 A}^{c}\end{array}$ & $\begin{array}{c}\text { averaged-1'C } \\
\text { (corrected) }^{d}\end{array}$ & \\
\hline 1 & 6.09 & 6.10 & 6.10 & 6.13 & 6.20 & 6.20 & 6.14 & 6.11 & 6.22 & 6.22 & 6.14 & 6.07 & 6.14 & 6.09 & $\begin{array}{l}6.09 \\
\end{array}$ & 6.08 & 6.12 & 5.48 & 5.69 \\
\hline 2 & 4.04 & 4.05 & 4.07 & 4.09 & 4.08 & 4.10 & 4.06 & 4.05 & 4.10 & 4.08 & 4.09 & 3.88 & 4.06 & 4.02 & 4.04 & 3.88 & 4.05 & 3.80 & 3.66 \\
\hline 3 & 3.77 & 3.77 & 3.78 & 3.82 & 3.93 & 3.93 & 3.81 & 3.78 & 3.95 & 3.95 & 3.82 & 4.04 & 3.82 & 3.76 & 3.76 & 4.04 & 3.82 & 3.61 & 3.77 \\
\hline 4 & 5.51 & 5.52 & 5.54 & 5.56 & 5.46 & 5.47 & 5.54 & 5.53 & 5.42 & 5.41 & 5.56 & 5.73 & 5.55 & 5.50 & 5.50 & 5.73 & 5.52 & 4.99 & 5.00 \\
\hline 5 & 4.24 & 4.24 & 4.26 & 4.29 & 4.33 & 4.32 & 4.28 & 4.26 & 4.32 & 4.33 & 4.29 & 4.18 & 4.29 & 4.23 & 4.23 & 4.17 & 4.26 & 3.97 & 3.98 \\
\hline \multirow[t]{2}{*}{6} & 5.44 & 5.45 & 5.45 & 5.45 & 5.35 & 5.35 & 5.44 & 5.44 & 5.31 & 5.31 & 5.45 & 5.57 & 5.44 & 5.44 & 5.45 & 5.57 & 5.43 & 4.92 & 4.64 \\
\hline & 4.00 & 4.00 & 4.03 & 4.04 & 4.03 & 4.03 & 4.03 & 4.02 & 4.02 & 4.02 & 4.04 & 4.08 & 4.04 & 4.00 & 4.00 & 4.07 & 4.02 & 3.77 & 3.72 \\
\hline $2^{\prime}, 6^{\prime}$ & 7.81 & 7.79 & 7.79 & 7.80 & 7.80 & 7.81 & 7.78 & 7.79 & 7.82 & 7.81 & 7.80 & 7.81 & 7.79 & 7.81 & 7.79 & 7.81 & 7.80 & 6.84 & 7.13 \\
\hline 3" & 7.98 & 7.98 & 8.09 & 8.09 & 8.15 & 8.16 & 8.09 & 8.09 & 8.13 & 8.13 & 8.11 & 7.73 & 8.11 & 7.91 & 7.91 & 7.73 & 8.03 & 7.02 & 6.59 \\
\hline 1"' & 4.58 & 4.58 & 4.63 & 4.56 & 4.58 & 4.59 & 4.56 & 4.63 & 4.52 & 4.52 & 4.56 & 4.57 & 4.56 & 4.54 & 4.54 & 4.57 & 4.57 & 4.22 & 4.33 \\
\hline 2"' & 3.59 & 3.59 & 3.79 & 3.99 & 4.03 & 4.02 & 3.98 & 3.79 & 4.10 & 4.10 & 4.03 & 3.57 & 4.03 & 3.52 & 3.52 & 3.57 & 3.76 & 3.56 & 3.66 \\
\hline \multirow[t]{2}{*}{ 3"' } & 2.23 & 2.24 & 2.19 & 2.19 & 2.39 & 2.40 & 2.18 & 2.19 & 2.46 & 2.47 & 2.27 & 2.11 & 2.26 & 2.20 & 2.20 & 2.11 & 2.26 & 2.34 & 2.28 \\
\hline & 2.51 & 2.51 & 2.34 & 2.44 & 2.40 & 2.41 & 2.44 & 2.34 & 2.41 & 2.40 & 2.49 & 2.61 & 2.49 & 2.64 & 2.65 & 2.61 & 2.47 & 2.52 & 2.60 \\
\hline
\end{tabular}

${ }^{a}$ Calculated using the GIAO method at the mPWIPW91/6-311+G(2d,p) level in MeOH $(P C M) .{ }^{b}$ Measured in $C D_{3} O D(500 \mathrm{MHz}) .{ }^{c}$ Averaged according to the Boltzmann distribution theory at $298 \mathrm{~K}$ based on relative Gibbs free energies. ${ }^{d}$ Linearly corrected for the experimental data.

Table S28. Calculated ${ }^{1} \mathrm{H}$ NMR chemical shifts of $\mathbf{1}^{\prime} \mathbf{D}$.

\begin{tabular}{|c|c|c|c|c|c|c|c|c|c|c|c|c|c|}
\hline \multirow[b]{2}{*}{ Position } & \multicolumn{12}{|c|}{ calculated $^{a}$} & \multirow{2}{*}{$\frac{\text { experimental }^{b}}{1}$} \\
\hline & 1'D-1 & 1'D-2 & 1'D-3 & 1'D-4 & 1'D-5 & 1'D-6 & 1'D-7 & 1'D-8 & 1'D-9 & 1'D-10 & $\begin{array}{c}\text { averaged- } \\
\mathbf{1 A}^{c}\end{array}$ & $\begin{array}{c}\text { averaged-1'D }^{\prime} \mathbf{D} \\
\text { (corrected) }^{d}\end{array}$ & \\
\hline 1 & 6.13 & 6.14 & 6.13 & 6.17 & 6.16 & 6.16 & 6.14 & 6.10 & 6.10 & 6.10 & 6.14 & 5.43 & 5.69 \\
\hline 2 & 4.07 & 4.09 & 4.05 & 4.08 & 4.09 & 4.08 & 4.06 & 3.91 & 4.04 & 4.05 & 4.07 & 3.77 & 3.66 \\
\hline 3 & 3.82 & 3.83 & 4.12 & 3.89 & 3.87 & 3.88 & 4.15 & 4.15 & 3.77 & 3.77 & 3.86 & 3.60 & 3.77 \\
\hline 4 & 5.56 & 5.57 & 5.88 & 5.55 & 5.57 & 5.56 & 5.90 & 5.87 & 5.53 & 5.54 & 5.60 & 5.00 & 5.00 \\
\hline 5 & 4.30 & 4.30 & 4.27 & 4.33 & 4.34 & 4.34 & 4.29 & 4.25 & 4.25 & 4.25 & 4.30 & 3.95 & 3.98 \\
\hline \multirow[t]{2}{*}{6} & 5.43 & 5.44 & 5.47 & 5.42 & 5.43 & 5.42 & 5.45 & 5.53 & 5.47 & 5.48 & 5.44 & 4.87 & 4.64 \\
\hline & 4.06 & 4.06 & 4.05 & 4.06 & 4.07 & 4.06 & 4.05 & 4.13 & 4.03 & 4.03 & 4.06 & 3.76 & 3.72 \\
\hline $2^{\prime}, 6^{\prime}$ & 7.79 & 7.80 & 7.78 & 7.79 & 7.80 & 7.79 & 7.79 & 7.77 & 7.82 & 7.80 & 7.79 & 6.77 & 7.13 \\
\hline 3" & 8.12 & 8.12 & 7.88 & 8.17 & 8.17 & 8.17 & 7.95 & 7.89 & 7.92 & 7.92 & 8.10 & 7.01 & 6.59 \\
\hline $1^{\prime \prime \prime}$ & 4.58 & 4.58 & 4.62 & 4.63 & 4.65 & 4.63 & 4.66 & 4.62 & 4.53 & 4.53 & 4.59 & 4.18 & 4.33 \\
\hline $2^{\prime \prime \prime}$ & 4.57 & 4.57 & 4.51 & 4.58 & 4.57 & 4.57 & 4.50 & 4.51 & 4.02 & 4.01 & 4.55 & 4.15 & 3.66 \\
\hline \multirow[t]{2}{*}{ 3"' } & 1.88 & 1.88 & 1.86 & 1.98 & 1.97 & 1.97 & 1.90 & 1.89 & 1.96 & 1.96 & 1.89 & 2.01 & 2.28 \\
\hline & 2.54 & 2.55 & 2.52 & 2.62 & 2.63 & 2.62 & 2.60 & 2.52 & 2.59 & 2.59 & 2.55 & 2.54 & 2.60 \\
\hline
\end{tabular}

${ }^{a}$ Calculated using the GIAO method at the mPWIPW91/6-311+G(2d,p) level in $\mathrm{MeOH}(\mathrm{PCM}) .{ }^{b}$ Measured in $\mathrm{CD}{ }_{3} \mathrm{OD}(500 \mathrm{MHz}) .{ }^{c}$ Averaged according to the

Boltzmann distribution theory at $298 \mathrm{~K}$ based on relative Gibbs free energies. ${ }^{d}$ Linearly corrected for the experimental data. 
Table S29. Calculated ${ }^{1} \mathrm{H}$ NMR chemical shifts of $\mathbf{1}^{\prime} \mathbf{E}$.

\begin{tabular}{|c|c|c|c|c|c|c|c|c|c|c|c|c|}
\hline \multirow[b]{2}{*}{ Position } & \multicolumn{11}{|c|}{ calculated $^{a}$} & \multirow{2}{*}{$\begin{array}{c}\text { experimental }^{b} \\
1\end{array}$} \\
\hline & $1^{\prime} \mathrm{E}-1$ & $1^{\prime} \mathrm{E}-2$ & $1^{\prime} \mathrm{E}-3$ & $1^{\prime}$ E-4 & $1^{\prime} \mathrm{E}-5$ & 1'E-6 & 1'E-7 & 1'E-8 & 1'E-9 & $\begin{array}{c}\text { averaged- } \\
\mathbf{1 A}^{c}\end{array}$ & $\begin{array}{c}\text { averaged-1'E } \\
{\text { (corrected) }{ }^{d}}^{\prime}\end{array}$ & \\
\hline 1 & 6.03 & 6.01 & 6.17 & 6.13 & 6.17 & 6.02 & 6.27 & 6.17 & 6.13 & 6.08 & 5.60 & 5.69 \\
\hline 2 & 4.06 & 4.04 & 4.13 & 4.11 & 3.90 & 4.03 & 3.72 & 4.12 & 4.11 & 4.06 & 3.83 & 3.66 \\
\hline 3 & 4.36 & 4.36 & 4.24 & 4.24 & 5.39 & 4.35 & 4.26 & 4.24 & 4.24 & 4.36 & 4.09 & 3.77 \\
\hline 4 & 5.50 & 5.50 & 5.33 & 5.32 & 4.46 & 5.49 & 5.46 & 5.33 & 5.32 & 5.39 & 4.99 & 5.00 \\
\hline 5 & 4.23 & 4.22 & 4.18 & 4.18 & 5.24 & 4.23 & 4.22 & 4.19 & 4.18 & 4.26 & 4.00 & 3.98 \\
\hline \multirow[t]{2}{*}{6} & 4.93 & 4.93 & 4.89 & 4.88 & 5.47 & 4.95 & 5.02 & 4.91 & 4.91 & 4.94 & 4.60 & 4.64 \\
\hline & 4.09 & 4.09 & 4.08 & 4.09 & 4.10 & 4.08 & 4.19 & 4.07 & 4.08 & 4.09 & 3.85 & 3.72 \\
\hline $2^{\prime}, 6^{\prime}$ & 7.87 & 7.86 & 7.88 & 7.86 & 7.82 & 7.87 & 7.74 & 7.88 & 7.87 & 7.86 & 7.16 & 7.13 \\
\hline 3" & 7.40 & 7.39 & 7.37 & 7.37 & 7.73 & 7.39 & 7.38 & 7.37 & 7.37 & 7.40 & 6.75 & 6.59 \\
\hline $1^{\prime \prime \prime}$ & 4.32 & 4.32 & 4.33 & 4.33 & 4.51 & 4.27 & 4.38 & 4.28 & 4.28 & 4.33 & 4.06 & 4.33 \\
\hline 2"' & 3.17 & 3.17 & 3.16 & 3.16 & 3.33 & 3.15 & 3.15 & 3.13 & 3.14 & 3.17 & 3.05 & 3.66 \\
\hline \multirow[t]{2}{*}{ 3"' } & 2.38 & 2.39 & 2.38 & 2.39 & 2.52 & 2.49 & 2.40 & 2.48 & 2.49 & 2.40 & 2.37 & 2.28 \\
\hline & 2.76 & 2.76 & 2.76 & 2.76 & 2.84 & 2.70 & 2.81 & 2.70 & 2.70 & 2.76 & 2.69 & 2.60 \\
\hline
\end{tabular}

${ }^{a}$ Calculated using the GIAO method at the mPWIPW91/6-311+G(2d,p) level in MeOH $(\mathrm{PCM}) .{ }^{b}$ Measured in $\mathrm{CD}{ }_{3} \mathrm{OD}(500 \mathrm{MHz}) .{ }^{c}$ Averaged according

to the Boltzmann distribution theory at $298 \mathrm{~K}$ based on relative Gibbs free energies. ${ }^{d}$ Linearly corrected for the experimental data.

Table S30. Calculated ${ }^{1} \mathrm{H}$ NMR chemical shifts of $\mathbf{1}^{\prime} \mathbf{F}$.

\begin{tabular}{|c|c|c|c|c|c|c|c|c|c|c|c|c|c|c|c|}
\hline \multirow[b]{2}{*}{ Position } & \multicolumn{14}{|c|}{ calculated $^{a}$} & \multirow{2}{*}{$\frac{\text { experimental }^{b}}{1}$} \\
\hline & $1^{\prime} \mathrm{F}-1$ & $1^{\prime} \mathrm{F}-2$ & $1^{\prime} F-3$ & 1'F-4 & 1'F-5 & 1'F-6 & 1'F-7 & $1^{\prime} \mathrm{F}-8$ & 1'F-9 & 1'F-10 & 1'F-11 & I'F-12 $^{\prime}$ & $\begin{array}{c}\text { averaged- } \\
\mathbf{1 A}^{c}\end{array}$ & $\begin{array}{c}\text { averaged-1'F }^{\prime} \text { (corrected) }^{d}\end{array}$ & \\
\hline 1 & 6.17 & 6.15 & 6.02 & 6.17 & 6.03 & 6.14 & 6.26 & 6.16 & 6.17 & 6.14 & 6.13 & 6.15 & 6.13 & 5.65 & 5.69 \\
\hline 2 & 4.15 & 4.13 & 4.06 & 4.14 & 4.08 & 4.12 & 3.73 & 4.15 & 4.16 & 4.12 & 4.11 & 4.13 & 4.11 & 3.89 & 3.66 \\
\hline 3 & 4.26 & 4.25 & 4.37 & 4.26 & 4.38 & 4.25 & 4.27 & 4.25 & 4.27 & 4.25 & 4.24 & 4.26 & 4.29 & 4.05 & 3.77 \\
\hline 4 & 5.37 & 5.36 & 5.53 & 5.35 & 5.53 & 5.35 & 5.48 & 5.36 & 5.36 & 5.35 & 5.35 & 5.36 & 5.41 & 5.03 & 5.00 \\
\hline 5 & 4.18 & 4.18 & 4.22 & 4.19 & 4.23 & 4.19 & 4.22 & 4.17 & 4.19 & 4.17 & 4.18 & 4.19 & 4.19 & 3.97 & 3.98 \\
\hline \multirow[t]{2}{*}{6} & 4.89 & 4.88 & 4.92 & 4.90 & 4.90 & 4.89 & 5.06 & 4.86 & 4.86 & 4.86 & 4.90 & 4.86 & 4.90 & 4.58 & 4.64 \\
\hline & 4.07 & 4.07 & 4.07 & 4.05 & 4.07 & 4.05 & 4.20 & 4.06 & 4.08 & 4.06 & 4.04 & 4.08 & 4.07 & 3.86 & 3.72 \\
\hline $2^{\prime}, 6^{\prime}$ & 7.86 & 7.86 & 7.85 & 7.86 & 7.84 & 7.86 & 7.75 & 7.86 & 7.86 & 7.85 & 7.87 & 7.86 & 7.86 & 7.17 & 7.13 \\
\hline 3" & 7.33 & 7.33 & 7.35 & 7.36 & 7.34 & 7.35 & 7.36 & 7.31 & 7.32 & 7.30 & 7.41 & 7.32 & 7.34 & 6.71 & 6.59 \\
\hline 1"' & 4.27 & 4.28 & 4.27 & 4.26 & 4.29 & 4.27 & 4.36 & 4.22 & 4.29 & 4.23 & 4.38 & 4.30 & 4.28 & 4.04 & 4.33 \\
\hline $2^{\prime \prime \prime}$ & 3.13 & 3.12 & 3.11 & 3.31 & 3.53 & 3.30 & 3.21 & 3.16 & 3.54 & 3.16 & 3.11 & 3.54 & 3.16 & 3.07 & 3.66 \\
\hline \multirow[t]{2}{*}{$3^{\prime \prime \prime}$} & 2.49 & 2.49 & 2.50 & 2.50 & 2.56 & 2.51 & 2.51 & 2.52 & 2.56 & 2.52 & 2.57 & 2.56 & 2.50 & 2.49 & 2.28 \\
\hline & 2.57 & 2.57 & 2.57 & 2.52 & 2.48 & 2.52 & 2.62 & 2.45 & 2.48 & 2.44 & 2.46 & 2.48 & 2.56 & 2.54 & 2.60 \\
\hline
\end{tabular}

${ }^{a}$ Calculated using the GIAO method at the mPWIPW91/6-311+G(2d,p) level in MeOH $(P C M) .{ }^{b}$ Measured in $\mathrm{CD}_{3} \mathrm{OD}(500 \mathrm{MHz}) .{ }^{c}$ Averaged according to the Boltzmann distribution theory at $298 \mathrm{~K}$ based on relative Gibbs free energies. ${ }^{d}$ Linearly corrected for the experimental data. 
Table S31. Calculated ${ }^{1} \mathrm{H}$ NMR chemical shifts of $\mathbf{1}^{\prime} \mathbf{G}$.

\begin{tabular}{|c|c|c|c|c|c|c|c|c|c|c|c|}
\hline \multirow[b]{2}{*}{ Position } & \multicolumn{10}{|c|}{ calculated $^{a}$} & \multirow{2}{*}{$\begin{array}{c}\text { experimental } \\
1\end{array}$} \\
\hline & 1'G-1 & 1'G-2 & I'G-3 $^{\prime}$ & I'G-4 $^{\prime}$ & $1^{\prime} G-5$ & 1'G-6 & 1'G-7 & 1'G-8 & $\begin{array}{c}\text { averaged- } \\
\mathbf{1 A}^{c}\end{array}$ & $\begin{array}{c}\text { averaged-1'G } \mathbf{G} \\
\text { (corrected) }^{d}\end{array}$ & \\
\hline 1 & 6.07 & 6.07 & 6.04 & 6.15 & 6.10 & 6.15 & 6.03 & 6.06 & 6.08 & 5.49 & 5.69 \\
\hline 2 & 4.06 & 4.07 & 4.06 & 4.19 & 4.15 & 4.18 & 4.05 & 4.06 & 4.09 & 3.76 & 3.66 \\
\hline 3 & 3.77 & 3.76 & 4.07 & 4.19 & 4.20 & 4.19 & 4.05 & 3.74 & 3.95 & 3.64 & 3.77 \\
\hline 4 & 5.62 & 5.63 & 5.91 & 5.24 & 5.34 & 5.22 & 5.89 & 5.62 & 5.58 & 5.06 & 5.00 \\
\hline 5 & 4.93 & 4.93 & 4.76 & 4.39 & 4.48 & 4.40 & 4.74 & 4.91 & 4.76 & 4.34 & 3.98 \\
\hline \multirow[t]{2}{*}{6} & 5.05 & 5.04 & 5.09 & 4.10 & 5.46 & 4.10 & 5.07 & 5.03 & 4.92 & 4.48 & 4.64 \\
\hline & 3.99 & 3.99 & 4.09 & 5.55 & 4.07 & 5.54 & 4.07 & 3.97 & 4.30 & 3.95 & 3.72 \\
\hline $2^{\prime}, 6^{\prime}$ & 7.80 & 7.79 & 7.78 & 7.77 & 7.81 & 7.76 & 7.78 & 7.79 & 7.79 & 6.97 & 7.13 \\
\hline $3^{\prime \prime}$ & 8.00 & 8.01 & 7.67 & 7.81 & 7.92 & 7.81 & 7.63 & 7.98 & 7.89 & 7.06 & 6.59 \\
\hline 1"' & 4.44 & 4.43 & 4.43 & 4.70 & 4.49 & 4.70 & 4.36 & 4.37 & 4.48 & 4.10 & 4.33 \\
\hline $2^{\prime \prime \prime}$ & 3.43 & 3.43 & 3.36 & 3.11 & 3.22 & 3.10 & 3.40 & 3.47 & 3.34 & 3.11 & 3.66 \\
\hline \multirow[t]{2}{*}{$3^{\prime \prime \prime}$} & 2.64 & 2.64 & 2.58 & 2.75 & 2.93 & 2.75 & 2.59 & 2.63 & 2.68 & 2.53 & 2.28 \\
\hline & 2.75 & 2.75 & 2.83 & 2.57 & 2.46 & 2.58 & 2.70 & 2.62 & 2.70 & 2.56 & 2.60 \\
\hline
\end{tabular}

${ }^{a}$ Calculated using the GIAO method at the mPWIPW91/6-311+G(2d,p) level in MeOH $(P C M) .{ }^{b}$ Measured in $\mathrm{CD}_{3} \mathrm{OD}(500 \mathrm{MHz}) .{ }^{c}$ Averaged according to the Boltzmann distribution theory at $298 \mathrm{~K}$ based on relative Gibbs free energies. ${ }^{d}$ Linearly corrected for the experimental data.

Table S32. Calculated ${ }^{1} \mathrm{H}$ NMR chemical shifts of $\mathbf{1}^{\prime} \mathbf{H}$.

\begin{tabular}{|c|c|c|c|c|c|c|c|c|c|c|c|c|}
\hline \multirow[b]{2}{*}{ Position } & \multicolumn{11}{|c|}{ calculated $^{a}$} & \multirow{2}{*}{$\begin{array}{c}\text { experimental }^{b} \\
1\end{array}$} \\
\hline & $1^{\prime} \mathrm{H}-1$ & $1^{\prime} \mathrm{H}-2$ & $\mathbf{1}^{\prime} \mathrm{H}-3$ & $1^{\prime} \mathrm{H}-4$ & $1^{\prime} \mathrm{H}-5$ & $1^{\prime} \mathrm{H}-6$ & $1^{\prime} \mathrm{H}-7$ & $1^{\prime} \mathrm{H}-8$ & 1'H-9 & $\begin{array}{c}\text { averaged- } \\
\mathbf{1 A}^{c}\end{array}$ & $\begin{array}{c}\text { averaged-1'H } \\
\text { (corrected) }^{d}\end{array}$ & \\
\hline 1 & 6.06 & 6.05 & 6.12 & 6.11 & 6.05 & 6.05 & 6.19 & 6.13 & 6.20 & 6.07 & 5.49 & 5.69 \\
\hline 2 & 4.06 & 4.05 & 4.20 & 4.19 & 4.04 & 4.06 & 3.72 & 4.19 & 3.72 & 4.07 & 3.75 & 3.66 \\
\hline 3 & 3.73 & 3.73 & 4.17 & 4.17 & 3.73 & 3.73 & 3.95 & 4.17 & 3.96 & 3.83 & 3.54 & 3.77 \\
\hline 4 & 5.65 & 5.64 & 5.18 & 5.18 & 5.64 & 5.65 & 5.84 & 5.21 & 5.84 & 5.56 & 5.04 & 5.00 \\
\hline 5 & 4.89 & 4.89 & 4.39 & 4.39 & 4.90 & 4.90 & 4.77 & 4.39 & 4.78 & 4.79 & 4.38 & 3.98 \\
\hline \multirow[t]{2}{*}{6} & 5.07 & 5.07 & 4.11 & 4.10 & 5.07 & 5.07 & 5.17 & 4.09 & 5.16 & 4.88 & 4.45 & 4.64 \\
\hline & 4.00 & 3.99 & 5.54 & 5.54 & 3.98 & 3.99 & 4.16 & 5.55 & 4.16 & 4.30 & 3.96 & 3.72 \\
\hline $2^{\prime}, 6^{\prime}$ & 7.79 & 7.80 & 7.75 & 7.75 & 7.80 & 7.79 & 7.78 & 7.75 & 7.78 & 7.79 & 6.97 & 7.13 \\
\hline 3" & 8.02 & 8.00 & 7.83 & 7.83 & 7.96 & 7.98 & 7.61 & 7.84 & 7.62 & 7.96 & 7.12 & 6.59 \\
\hline $1^{\prime \prime \prime}$ & 4.36 & 4.35 & 4.75 & 4.76 & 4.32 & 4.32 & 4.44 & 4.69 & 4.44 & 4.43 & 4.07 & 4.33 \\
\hline $2^{\prime \prime \prime}$ & 3.35 & 3.34 & 3.15 & 3.15 & 3.44 & 3.45 & 3.32 & 3.18 & 3.32 & 3.31 & 3.10 & 3.66 \\
\hline \multirow[t]{2}{*}{ 3"' } & 2.53 & 2.53 & 2.50 & 2.50 & 2.61 & 2.61 & 2.45 & 2.65 & 2.45 & 2.53 & 2.42 & 2.28 \\
\hline & 2.98 & 2.98 & 2.76 & 2.76 & 2.88 & 2.88 & 2.93 & 2.73 & 2.91 & 2.93 & 2.76 & 2.60 \\
\hline
\end{tabular}

${ }^{a}$ Calculated using the GIAO method at the mPWIPW91/6-311+G(2d,p) level in $\mathrm{MeOH}(\mathrm{PCM}) .{ }^{b}$ Measured in $\mathrm{CD}_{3} \mathrm{OD}(500 \mathrm{MHz}) .{ }^{c}$ Averaged according

to the Boltzmann distribution theory at $298 \mathrm{~K}$ based on relative Gibbs free energies. ${ }^{d}$ Linearly corrected for the experimental data. 
Table S33. Calculated ${ }^{13} \mathrm{C}$ NMR chemical shifts of $\mathbf{1 A}$.

\begin{tabular}{|c|c|c|c|c|c|c|c|c|c|c|c|c|c|c|}
\hline \multirow[b]{2}{*}{ Position } & \multicolumn{13}{|c|}{ calculated $^{a}$} & \multirow{2}{*}{$\begin{array}{c}\text { experimental }^{b} \\
1\end{array}$} \\
\hline & 1A-1 & 1A-2 & $1 \mathrm{~A}-3$ & $1 \mathrm{~A}-4$ & $1 A-5$ & $1 \mathrm{~A}-6$ & 1A-7 & 1A-8 & 1A-9 & $1 \mathrm{~A}-10$ & 1A-11 & averaged- $\mathbf{1} \mathbf{A}^{c}$ & $\begin{array}{l}\text { averaged-1A } \text { (corrected) }^{d}\end{array}$ & \\
\hline 1 & 93.6 & 93.6 & 93.6 & 91.7 & 93.7 & 91.7 & 96.2 & 93.6 & 96.1 & 93.7 & 93.7 & 93.6 & 94.1 & 95.6 \\
\hline 2 & 74.1 & 74.1 & 74.1 & 69.9 & 74.1 & 69.9 & 73.9 & 74.1 & 73.9 & 74.0 & 73.8 & 73.7 & 75.1 & 74.1 \\
\hline 3 & 74.3 & 74.3 & 74.3 & 72.2 & 74.3 & 72.1 & 74.8 & 74.4 & 74.8 & 74.3 & 74.3 & 74.1 & 75.5 & 75.9 \\
\hline 4 & 75.0 & 75.0 & 75.0 & 84.7 & 74.9 & 84.7 & 72.1 & 74.9 & 72.2 & 75.0 & 75.6 & 75.8 & 77.1 & 75.4 \\
\hline 5 & 71.7 & 71.7 & 71.6 & 68.2 & 71.7 & 68.2 & 69.9 & 71.6 & 69.9 & 71.6 & 71.7 & 71.3 & 72.8 & 71.2 \\
\hline 6 & 63.8 & 63.8 & 63.8 & 62.3 & 63.6 & 62.2 & 64.2 & 63.7 & 64.2 & 63.9 & 64.3 & 63.7 & 65.5 & 66.5 \\
\hline $1^{\prime}$ & 119.5 & 119.5 & 119.7 & 119.4 & 119.4 & 119.7 & 118.7 & 119.7 & 118.8 & 119.4 & 119.3 & 119.5 & 119.0 & 120.4 \\
\hline $2^{\prime}, 6^{\prime}$ & 110.4 & 110.4 & 110.4 & 110.0 & 110.5 & 110.0 & 110.4 & 110.3 & 110.3 & 110.5 & 110.5 & 110.4 & 110.2 & 110.5 \\
\hline $3^{\prime}, 5^{\prime}$ & 145.8 & 145.8 & 146.0 & 145.7 & 145.9 & 145.7 & 145.0 & 146.0 & 145.0 & 145.8 & 145.9 & 145.8 & 144.2 & 146.5 \\
\hline $4^{\prime}$ & 140.4 & 140.5 & 140.0 & 138.9 & 140.5 & 139.0 & 139.0 & 140.0 & 139.1 & 140.5 & 140.5 & 140.2 & 138.8 & 140.4 \\
\hline $7^{\prime}$ & 167.6 & 167.6 & 167.4 & 167.6 & 167.6 & 167.4 & 171.3 & 167.5 & 171.1 & 167.5 & 167.5 & 167.8 & 165.3 & 166.7 \\
\hline 1" & 114.2 & 114.2 & 114.3 & 114.0 & 115.5 & 113.8 & 115.3 & 115.5 & 115.3 & 113.8 & 112.2 & 114.2 & 114.0 & 115.4 \\
\hline $2^{\prime \prime}$ & 125.4 & 125.4 & 125.4 & 127.0 & 125.3 & 127.0 & 124.9 & 125.3 & 124.9 & 125.1 & 126.3 & 125.5 & 124.8 & 123.9 \\
\hline $3^{\prime \prime}$ & 106.4 & 106.4 & 106.4 & 112.3 & 106.5 & 112.2 & 107.9 & 106.5 & 107.9 & 106.0 & 105.9 & 107.0 & 107.1 & 108.4 \\
\hline $4^{\prime \prime}$ & 147.0 & 147.0 & 147.0 & 145.7 & 146.8 & 146.0 & 146.6 & 146.8 & 146.6 & 146.9 & 147.9 & 146.9 & 145.3 & 146.1 \\
\hline $5^{\prime \prime}$ & 135.6 & 135.6 & 135.6 & 134.0 & 135.6 & 134.9 & 136.7 & 135.6 & 136.7 & 135.3 & 135.5 & 135.5 & 134.3 & 136.2 \\
\hline 6" & 142.4 & 142.4 & 142.5 & 140.1 & 142.2 & 140.3 & 142.4 & 142.2 & 142.5 & 142.8 & 142.9 & 142.2 & 140.8 & 142.6 \\
\hline 7" & 179.2 & 179.2 & 179.2 & 173.3 & 179.3 & 173.3 & 172.4 & 179.3 & 172.5 & 179.2 & 180.0 & 178.3 & 175.4 & 169.4 \\
\hline 1"' & 44.7 & 44.7 & 44.8 & 47.2 & 45.4 & 47.2 & 43.5 & 45.5 & 43.3 & 47.5 & 44.8 & 45.0 & 47.6 & 46.4 \\
\hline $2^{\prime \prime \prime}$ & 47.3 & 47.3 & 47.4 & 45.8 & 47.4 & 45.9 & 48.7 & 47.4 & 48.7 & 47.1 & 47.3 & 47.3 & 49.8 & 48.3 \\
\hline 3"' & 34.6 & 34.6 & 34.5 & 33.4 & 35.1 & 33.5 & 32.8 & 35.1 & 32.8 & 34.7 & 34.2 & 34.4 & 37.5 & 37.8 \\
\hline 4"' & 110.3 & 110.3 & 110.3 & 109.7 & 108.9 & 109.8 & 109.6 & 108.9 & 109.6 & 110.6 & 110.3 & 110.1 & 110.0 & 108.2 \\
\hline 5"' & 77.4 & 77.5 & 77.5 & 77.8 & 80.4 & 77.7 & 77.2 & 80.4 & 77.2 & 78.6 & 78.0 & 77.7 & 78.9 & 80.1 \\
\hline 6"' & 177.9 & 177.9 & 178.0 & 177.1 & 172.9 & 177.3 & 178.5 & 173.0 & 178.4 & 176.9 & 178.2 & 177.6 & 174.7 & 172.7 \\
\hline $7^{\prime \prime \prime}$ & 177.6 & 177.6 & 177.5 & 177.5 & 177.5 & 177.6 & 177.1 & 177.5 & 177.1 & 177.6 & 177.0 & 177.5 & 174.6 & 173.7 \\
\hline
\end{tabular}

Boltzmann distribution theory at $298 \mathrm{~K}$ based on relative Gibbs free energies. ${ }^{d}$ Linearly corrected for the experimental data. 
Table S34. Calculated ${ }^{13} \mathrm{C}$ NMR chemical shifts of $\mathbf{1 B}$.

\begin{tabular}{|c|c|c|c|c|c|c|c|c|c|c|c|c|c|c|c|c|c|c|c|c|}
\hline \multirow[b]{2}{*}{ Position } & \multicolumn{19}{|c|}{ calculated $^{a}$} & \multirow{2}{*}{$\begin{array}{c}\text { experimental }^{b} \\
1\end{array}$} \\
\hline & 1B-1 & 1B-2 & $1 B-3$ & $1 B-4$ & $1 B-5$ & 1B-6 & 1B-7 & 1B-8 & 1B-9 & $1 B-10$ & 1B-11 & 1B-12 & 1B-13 & $1 B-14$ & 1B-15 & 1B-16 & 1B-17 & averaged-1 $\mathbf{B}^{c}$ & $\begin{array}{l}\text { averaged-1B } \\
\text { (corrected) }^{d}\end{array}$ & \\
\hline 1 & 94.1 & 94.0 & 94.1 & 94.0 & 94.0 & 94.0 & 96.3 & 94.1 & 96.3 & 96.4 & 94.0 & 91.5 & 96.4 & 94.0 & 91.4 & 100.7 & 100.6 & 94.4 & 94.7 & 95.6 \\
\hline 2 & 73.9 & 73.9 & 73.8 & 73.9 & 73.9 & 74.0 & 74.2 & 73.8 & 74.2 & 74.1 & 73.9 & 69.3 & 74.1 & 73.9 & 69.3 & 76.2 & 76.2 & 73.8 & 74.7 & 74.1 \\
\hline 3 & 74.3 & 74.3 & 74.3 & 74.3 & 74.3 & 74.2 & 74.8 & 74.4 & 74.8 & 74.9 & 74.3 & 71.3 & 74.9 & 74.3 & 71.4 & 75.3 & 75.3 & 74.3 & 75.2 & 75.9 \\
\hline 4 & 75.1 & 75.1 & 74.9 & 74.9 & 74.9 & 74.9 & 71.9 & 75.0 & 71.9 & 72.3 & 74.9 & 85.0 & 72.3 & 74.9 & 85.0 & 73.3 & 73.3 & 75.0 & 75.9 & 75.4 \\
\hline 5 & 71.5 & 71.5 & 71.5 & 71.6 & 71.5 & 71.6 & 69.7 & 71.5 & 69.7 & 69.9 & 71.6 & 68.2 & 69.9 & 71.6 & 68.2 & 72.7 & 72.7 & 71.2 & 72.2 & 71.2 \\
\hline 6 & 63.9 & 63.9 & 63.9 & 63.9 & 63.8 & 63.9 & 63.9 & 63.8 & 63.9 & 63.7 & 63.8 & 62.2 & 63.7 & 63.8 & 62.1 & 65.2 & 65.2 & 63.9 & 65.1 & 66.5 \\
\hline $1^{\prime}$ & 118.8 & 119.2 & 118.8 & 118.8 & 119.2 & 119.3 & 119.2 & 118.8 & 119.1 & 119.2 & 118.8 & 121.1 & 119.1 & 119.2 & 121.1 & 119.5 & 119.5 & 119.1 & 118.6 & 120.4 \\
\hline $2^{\prime}, 6^{\prime}$ & 110.9 & 110.5 & 110.9 & 110.8 & 110.5 & 110.5 & 110.0 & 110.9 & 110.0 & 110.1 & 110.9 & 109.0 & 110.0 & 110.5 & 109.0 & 110.1 & 110.1 & 110.6 & 110.3 & 110.5 \\
\hline $3^{\prime}, 5^{\prime}$ & 146.2 & 146.1 & 146.2 & 146.2 & 146.1 & 146.1 & 145.3 & 146.2 & 145.3 & 145.3 & 146.2 & 144.3 & 145.3 & 146.1 & 144.3 & 145.5 & 145.5 & 146.0 & 144.7 & 146.5 \\
\hline $4^{\prime}$ & 140.7 & 140.4 & 140.7 & 140.7 & 140.4 & 140.4 & 139.5 & 140.7 & 139.2 & 139.6 & 140.7 & 137.5 & 139.3 & 140.4 & 137.3 & 140.1 & 140.1 & 140.3 & 139.2 & 140.4 \\
\hline $7^{\prime}$ & 167.1 & 167.0 & 167.1 & 167.1 & 167.0 & 167.0 & 171.4 & 167.1 & 171.5 & 171.5 & 167.0 & 169.6 & 171.6 & 167.0 & 169.6 & 172.4 & 172.5 & 167.8 & 165.9 & 166.7 \\
\hline 1" & 108.1 & 108.1 & 114.8 & 114.0 & 114.8 & 114.0 & 117.4 & 114.4 & 117.5 & 110.3 & 113.3 & 115.5 & 110.3 & 113.2 & 110.1 & 109.9 & 109.9 & 112.0 & 111.7 & 115.4 \\
\hline $2^{\prime \prime}$ & 128.0 & 128.1 & 126.0 & 125.8 & 126.0 & 125.8 & 126.5 & 126.1 & 126.6 & 127.2 & 126.3 & 127.6 & 127.3 & 126.3 & 128.7 & 127.1 & 127.1 & 126.9 & 126.2 & 123.9 \\
\hline 3" & 107.4 & 107.5 & 107.8 & 106.2 & 107.8 & 106.2 & 109.6 & 108.5 & 109.6 & 109.7 & 106.2 & 113.0 & 109.7 & 106.2 & 112.6 & 108.4 & 108.4 & 107.8 & 107.6 & 108.4 \\
\hline 4" & 147.9 & 147.9 & 146.9 & 146.7 & 146.9 & 146.7 & 146.3 & 147.1 & 146.2 & 146.9 & 146.8 & 145.4 & 146.8 & 146.8 & 146.2 & 147.2 & 147.2 & 147.1 & 145.8 & 146.1 \\
\hline $5^{\prime \prime}$ & 134.9 & 134.9 & 135.3 & 134.5 & 135.3 & 134.6 & 135.5 & 135.3 & 135.5 & 135.4 & 134.6 & 136.0 & 135.3 & 134.6 & 136.6 & 135.7 & 135.7 & 135.1 & 134.1 & 136.2 \\
\hline 6" & 143.3 & 143.4 & 141.4 & 143.4 & 141.4 & 143.4 & 140.1 & 141.2 & 140.1 & 141.6 & 143.2 & 141.4 & 141.7 & 143.2 & 142.3 & 142.9 & 142.9 & 142.5 & 141.3 & 142.6 \\
\hline $7^{\prime \prime}$ & 178.7 & 178.8 & 179.1 & 179.7 & 179.1 & 179.7 & 172.2 & 178.9 & 172.2 & 172.1 & 179.8 & 173.3 & 172.1 & 179.8 & 173.1 & 174.8 & 174.8 & 177.9 & 175.7 & 169.4 \\
\hline 1"' & 48.8 & 48.9 & 48.8 & 48.1 & 48.9 & 48.2 & 47.0 & 48.8 & 47.0 & 46.3 & 48.8 & 52.3 & 46.3 & 48.8 & 51.7 & 47.3 & 47.2 & 48.6 & 50.2 & 46.4 \\
\hline $2^{\prime \prime \prime}$ & 46.6 & 46.7 & 47.8 & 45.8 & 47.8 & 45.8 & 48.5 & 46.8 & 48.5 & 48.1 & 46.4 & 46.3 & 48.1 & 46.4 & 46.2 & 45.9 & 45.9 & 46.9 & 48.6 & 48.3 \\
\hline 3"' & 34.6 & 34.5 & 36.1 & 34.4 & 36.0 & 34.4 & 35.4 & 33.4 & 35.4 & 33.6 & 34.5 & 36.4 & 33.5 & 34.5 & 34.6 & 34.3 & 34.3 & 34.8 & 36.9 & 37.8 \\
\hline 4"' & 109.1 & 109.1 & 108.4 & 108.0 & 108.3 & 108.0 & 108.2 & 108.8 & 108.2 & 107.8 & 108.4 & 107.8 & 107.8 & 108.3 & 107.6 & 108.5 & 108.5 & 108.5 & 108.4 & 108.2 \\
\hline 5"' & 80.9 & 80.9 & 80.9 & 80.5 & 80.8 & 80.5 & 80.3 & 82.1 & 80.3 & 80.8 & 81.2 & 80.8 & 80.7 & 81.2 & 81.4 & 80.6 & 80.6 & 80.8 & 81.5 & 80.1 \\
\hline 6"' & 175.0 & 175.0 & 175.7 & 179.0 & 175.7 & 179.0 & 176.0 & 174.7 & 176.0 & 174.8 & 175.0 & 175.4 & 174.8 & 175.0 & 174.7 & 175.2 & 175.2 & 175.9 & 173.8 & 172.7 \\
\hline $7^{\prime \prime \prime}$ & 176.5 & 176.4 & 176.3 & 176.8 & 176.2 & 176.8 & 175.7 & 176.4 & 175.7 & 176.0 & 176.8 & 176.9 & 176.0 & 176.7 & 177.2 & 177.3 & 177.3 & 176.5 & 174.3 & 173.7 \\
\hline
\end{tabular}

Calculated using the GIAO method at the mPWIPW91/6-311+G(2d,p) level in $\mathrm{MeOH}(\mathrm{PCM}) .{ }^{b}$ Measured in $\mathrm{CD}{ }_{3} \mathrm{OD}(125 \mathrm{MHz}) .{ }^{c}$ Averaged according to the Boltzmann distribution theory at $298 \mathrm{~K}$ based on relative

Gibbs free energies. ${ }^{d}$ Linearly corrected for the experimental data. 
Table S35. Calculated ${ }^{13} \mathrm{C}$ NMR chemical shifts of $\mathbf{1 C}$.

\begin{tabular}{|c|c|c|c|c|c|c|c|c|c|c|c|}
\hline \multirow[b]{2}{*}{ Position } & \multicolumn{10}{|c|}{ calculated $^{a}$} & \multirow{2}{*}{$\begin{array}{c}\text { experimental }^{b} \\
1\end{array}$} \\
\hline & 1C-1 & 1C-2 & $1 \mathrm{C}-3$ & 1C-4 & 1C-5 & 1C-6 & 1C-7 & 1C-8 & averaged-1 $\mathbf{C}^{c}$ & $\begin{array}{l}\text { averaged-1C } \\
{\text { (corrected) })^{d}}^{\text {cot }}\end{array}$ & \\
\hline 1 & 93.6 & 93.6 & 93.6 & 93.5 & 95.0 & 93.5 & 93.5 & 93.5 & 93.6 & 93.6 & 95.6 \\
\hline 2 & 73.7 & 73.7 & 73.7 & 73.7 & 74.8 & 73.7 & 73.7 & 73.7 & 73.8 & 74.1 & 74.1 \\
\hline 3 & 75.5 & 75.5 & 75.5 & 75.4 & 74.0 & 75.5 & 75.5 & 75.5 & 75.4 & 75.7 & 75.9 \\
\hline 4 & 79.3 & 79.3 & 79.1 & 79.2 & 80.3 & 79.3 & 79.1 & 79.3 & 79.3 & 79.5 & 75.4 \\
\hline 5 & 71.6 & 71.5 & 71.5 & 71.5 & 71.2 & 71.6 & 71.5 & 71.5 & 71.5 & 71.9 & 71.2 \\
\hline 6 & 62.5 & 62.4 & 62.4 & 62.3 & 62.7 & 62.5 & 62.4 & 62.4 & 62.4 & 63.0 & 66.5 \\
\hline $1^{\prime}$ & 120.3 & 120.3 & 120.3 & 120.3 & 119.8 & 120.3 & 120.3 & 120.3 & 120.3 & 119.6 & 120.4 \\
\hline $2^{\prime}, 6^{\prime}$ & 109.7 & 109.6 & 109.7 & 109.6 & 109.8 & 109.7 & 109.7 & 109.6 & 109.6 & 109.2 & 110.5 \\
\hline 3', 5' & 145.3 & 145.4 & 145.3 & 145.5 & 145.3 & 145.3 & 145.3 & 145.4 & 145.3 & 144.1 & 146.5 \\
\hline $4^{\prime}$ & 138.4 & 138.7 & 138.4 & 138.7 & 138.6 & 138.4 & 138.4 & 138.7 & 138.5 & 137.5 & 140.4 \\
\hline $7^{\prime}$ & 168.6 & 168.3 & 168.5 & 168.3 & 168.3 & 168.6 & 168.5 & 168.4 & 168.5 & 166.8 & 166.7 \\
\hline 1" & 119.2 & 119.2 & 120.3 & 120.3 & 119.6 & 118.8 & 119.7 & 118.8 & 119.4 & 118.8 & 115.4 \\
\hline 2" & 120.4 & 120.4 & 120.7 & 120.6 & 120.3 & 120.0 & 120.3 & 120.0 & 120.4 & 119.8 & 123.9 \\
\hline 3" & 115.9 & 115.8 & 115.7 & 115.7 & 115.9 & 115.7 & 115.5 & 115.6 & 115.8 & 115.3 & 108.4 \\
\hline 4" & 145.2 & 145.1 & 144.8 & 144.8 & 145.3 & 145.1 & 144.7 & 145.1 & 145.1 & 143.9 & 146.1 \\
\hline 5" & 137.5 & 137.6 & 137.5 & 137.5 & 137.7 & 137.4 & 137.3 & 137.4 & 137.5 & 136.5 & 136.2 \\
\hline 6" & 140.3 & 140.3 & 140.4 & 140.4 & 140.8 & 140.6 & 140.6 & 140.6 & 140.4 & 139.3 & 142.6 \\
\hline 7" & 175.2 & 175.3 & 175.2 & 175.2 & 175.4 & 175.2 & 175.1 & 175.2 & 175.2 & 173.4 & 169.4 \\
\hline 1"' & 48.0 & 48.0 & 49.2 & 49.2 & 48.2 & 50.7 & 51.6 & 50.7 & 48.5 & 49.4 & 46.4 \\
\hline $2^{2 \prime \prime}$ & 48.6 & 48.5 & 48.5 & 48.5 & 48.6 & 48.7 & 48.5 & 48.6 & 48.6 & 49.5 & 48.3 \\
\hline 3"' & 34.3 & 34.3 & 34.9 & 34.8 & 34.2 & 35.0 & 35.6 & 34.9 & 34.5 & 35.7 & 37.8 \\
\hline $4^{\prime \prime \prime}$ & 108.3 & 108.3 & 107.1 & 107.2 & 108.3 & 108.0 & 107.1 & 108.0 & 108.0 & 107.6 & 108.2 \\
\hline $5^{\prime \prime \prime}$ & 77.4 & 77.4 & 79.8 & 79.8 & 77.2 & 78.7 & 81.0 & 78.6 & 78.0 & 78.3 & 80.1 \\
\hline 6"' & 177.8 & 177.8 & 171.8 & 171.8 & 177.8 & 176.6 & 172.5 & 176.6 & 176.3 & 174.4 & 172.7 \\
\hline 7"' & 177.3 & 177.3 & 177.3 & 177.3 & 177.3 & 177.3 & 177.3 & 177.3 & 177.3 & 175.4 & 173.7 \\
\hline
\end{tabular}


Table S36. Calculated ${ }^{13} \mathrm{C}$ NMR chemical shifts of $\mathbf{1 D}$.

\begin{tabular}{|c|c|c|c|c|c|c|c|c|c|c|c|c|}
\hline \multirow[b]{2}{*}{ Position } & \multicolumn{11}{|c|}{ calculated $^{a}$} & \multirow{2}{*}{$\begin{array}{c}\text { experimental }^{b} \\
1 \\
\end{array}$} \\
\hline & 1D-1 & 1D-2 & 1D-3 & 1D-4 & 1D-5 & 1D-6 & 1D-7 & 1D-8 & 1D-9 & averaged-1 $\mathbf{D}^{c}$ & $\begin{array}{l}\text { averaged-1D } \\
\text { (corrected) }^{d}\end{array}$ & \\
\hline 1 & 93.4 & 93.5 & 93.4 & 93.5 & 93.5 & 93.4 & 93.5 & $\begin{array}{l}93.4 \\
\end{array}$ & 93.5 & 93.5 & $\begin{array}{l}92.9 \\
\end{array}$ & 95.6 \\
\hline 2 & 73.8 & 73.8 & 73.9 & 73.9 & 73.9 & 73.9 & 73.9 & 73.9 & 73.9 & 73.8 & 73.5 & 74.1 \\
\hline 3 & 75.2 & 75.2 & 75.1 & 75.1 & 75.1 & 75.1 & 75.1 & 75.1 & 75.1 & 75.1 & 74.8 & 75.9 \\
\hline 4 & 79.3 & 79.2 & 79.4 & 79.3 & 79.3 & 79.3 & 79.2 & 79.3 & 79.2 & 79.3 & 78.9 & 75.4 \\
\hline 5 & 71.5 & 71.5 & 71.7 & 71.7 & 71.7 & 71.6 & 71.7 & 71.6 & 71.6 & 71.6 & 71.3 & 71.2 \\
\hline 6 & 62.7 & 62.7 & 62.8 & 62.9 & 62.8 & 62.7 & 62.8 & 62.7 & 62.7 & 62.7 & 62.6 & 66.5 \\
\hline $1^{\prime}$ & 120.1 & 120.1 & 120.1 & 120.2 & 120.1 & 120.1 & 120.1 & 120.2 & 120.2 & 120.1 & 119.2 & 120.4 \\
\hline $2^{\prime}, 6^{\prime}$ & 109.7 & 109.6 & 109.7 & 109.6 & 109.7 & 109.7 & 109.6 & 109.6 & 109.6 & 109.7 & 108.9 & 110.5 \\
\hline $3^{\prime}, 5^{\prime}$ & 145.4 & 145.3 & 145.4 & 145.4 & 145.4 & 145.4 & 145.4 & 145.3 & 145.3 & 145.4 & 144.2 & 146.5 \\
\hline $4^{\prime}$ & 138.6 & 138.5 & 138.6 & 138.5 & 138.6 & 138.5 & 138.6 & 138.5 & 138.5 & 138.6 & 137.5 & 140.4 \\
\hline $7^{\prime}$ & 168.2 & 168.3 & 168.3 & 168.4 & 168.3 & 168.3 & 168.4 & 168.4 & 168.4 & 168.3 & 166.8 & 166.7 \\
\hline 1" & 119.9 & 119.8 & 119.6 & 119.6 & 119.9 & 119.8 & 119.8 & 119.7 & 119.6 & 119.8 & 118.9 & 115.4 \\
\hline 2" & 121.0 & 121.1 & 120.6 & 120.6 & 120.8 & 120.4 & 120.9 & 120.4 & 120.5 & 120.9 & 120.0 & 123.9 \\
\hline 3" & 116.2 & 116.2 & 115.0 & 115.1 & 114.9 & 114.8 & 114.9 & 114.8 & 115.1 & 115.9 & 115.0 & 108.4 \\
\hline 4" & 144.8 & 144.8 & 144.7 & 144.7 & 144.5 & 144.7 & 144.5 & 144.7 & 144.8 & 144.7 & 143.5 & 146.1 \\
\hline 5" & 138.0 & 138.0 & 137.8 & 137.8 & 137.7 & 137.7 & 137.7 & 137.7 & 137.7 & 137.9 & 136.8 & 136.2 \\
\hline 6" & 141.1 & 141.1 & 143.1 & 143.2 & 142.6 & 142.8 & 142.6 & 142.8 & 143.1 & 141.7 & 140.6 & 142.6 \\
\hline 7" & 175.1 & 175.1 & 175.6 & 175.5 & 175.6 & 175.6 & 175.6 & 175.6 & 175.5 & 175.2 & 173.7 & 169.4 \\
\hline 1"' & 52.7 & 52.7 & 52.1 & 52.1 & 52.8 & 51.2 & 52.7 & 51.2 & 50.4 & 52.5 & 52.5 & 46.4 \\
\hline $2^{\prime \prime \prime}$ & 46.9 & 46.9 & 47.2 & 47.3 & 46.9 & 47.1 & 46.9 & 47.2 & 46.5 & 47.0 & 47.0 & 48.3 \\
\hline 3"' & 37.0 & 37.0 & 34.7 & 34.7 & 38.5 & 37.3 & 38.5 & 37.3 & 33.9 & 36.5 & 36.7 & 37.8 \\
\hline 4"' & 107.3 & 107.3 & 106.4 & 106.4 & 106.3 & 107.1 & 106.3 & 107.1 & 107.0 & 107.0 & 106.3 & 108.2 \\
\hline $5^{\prime \prime \prime}$ & 81.1 & 81.1 & 80.9 & 80.9 & 80.0 & 81.1 & 80.1 & 81.1 & 81.3 & 81.0 & 80.6 & 80.1 \\
\hline 6"' & 175.1 & 175.1 & 178.9 & 178.8 & 179.2 & 177.7 & 179.2 & 177.7 & 175.4 & 176.2 & 174.6 & 172.7 \\
\hline 7"' & 176.6 & 176.6 & 177.1 & 177.2 & 177.0 & 177.2 & 177.0 & 177.2 & 177.3 & 176.7 & 175.2 & 173.7 \\
\hline
\end{tabular}


Table S37. Calculated ${ }^{13} \mathrm{C}$ NMR chemical shifts of $\mathbf{1 E}$.

\begin{tabular}{|c|c|c|c|c|c|c|c|c|c|c|c|c|c|c|c|c|c|}
\hline \multirow[b]{2}{*}{ Position } & \multicolumn{16}{|c|}{ calculated $^{a}$} & \multirow{2}{*}{$\begin{array}{c}\text { experimental }^{b} \\
1\end{array}$} \\
\hline & 1E-1 & 1E-2 & $1 E-3$ & $1 \mathrm{E}-4$ & $1 E-5$ & 1E-6 & 1E-7 & 1E-8 & 1E-9 & 1E-10 & 1E-11 & 1E-12 & 1E-13 & 1E-14 & averaged-1 $\mathbf{1} \mathrm{E}^{c}$ & $\begin{array}{l}\text { averaged-1E } \\
\text { (corrected) }^{d}\end{array}$ & \\
\hline 1 & 91.7 & 92.1 & 92.2 & 92.2 & 91.6 & 92.2 & 91.7 & 91.6 & 92.2 & 92.2 & 92.0 & 92.1 & 92.2 & 92.2 & 92.0 & 91.7 & 95.6 \\
\hline 2 & 71.2 & 70.8 & 70.8 & 70.9 & 71.2 & 70.9 & 71.2 & 71.3 & 70.8 & 70.8 & 70.6 & 70.7 & 70.8 & 70.8 & 71.0 & 71.2 & 74.1 \\
\hline 3 & 75.7 & 72.2 & 72.3 & 72.2 & 75.6 & 72.2 & 75.6 & 75.5 & 72.2 & 72.2 & 71.9 & 72.0 & 72.1 & 72.1 & 73.4 & 73.6 & 75.9 \\
\hline 4 & 85.1 & 88.8 & 88.8 & 87.9 & 85.1 & 87.9 & 85.2 & 85.2 & 87.9 & 87.9 & 88.6 & 88.7 & 88.1 & 88.1 & 87.2 & 87.1 & 75.4 \\
\hline 5 & 70.8 & 71.1 & 71.2 & 70.8 & 70.8 & 70.8 & 70.7 & 70.7 & 70.9 & 70.8 & 71.0 & 71.0 & 70.8 & 70.8 & 70.9 & 71.2 & 71.2 \\
\hline 6 & 65.0 & 68.2 & 68.3 & 66.4 & 65.0 & 66.4 & 65.1 & 65.1 & 66.4 & 66.4 & 68.0 & 68.1 & 66.6 & 66.6 & 66.5 & 66.8 & 66.5 \\
\hline $1^{\prime}$ & 120.8 & 120.5 & 120.4 & 120.4 & 120.7 & 120.6 & 120.8 & 120.7 & 120.3 & 120.5 & 120.1 & 120.0 & 120.5 & 120.3 & 120.5 & 119.7 & 120.4 \\
\hline $2^{\prime}, 6^{\prime}$ & 109.4 & 109.1 & 109.3 & 109.2 & 109.4 & 109.0 & 109.4 & 109.5 & 109.2 & 109.1 & 109.5 & 109.5 & 109.1 & 109.3 & 109.3 & 108.6 & 110.5 \\
\hline $3^{\prime}, 5^{\prime}$ & 145.0 & 144.8 & 144.6 & 144.5 & 145.0 & 144.7 & 144.9 & 145.0 & 144.6 & 144.8 & 145.3 & 144.8 & 144.8 & 144.6 & 144.8 & 143.3 & 146.5 \\
\hline $4^{\prime}$ & 138.6 & 138.7 & 139.0 & 138.7 & 138.7 & 138.6 & 138.6 & 138.7 & 138.7 & 138.6 & 138.6 & 138.7 & 138.6 & 138.7 & 138.7 & 137.4 & 140.4 \\
\hline $7^{\prime}$ & 168.9 & 168.8 & 168.7 & 168.7 & 168.7 & 168.8 & 168.9 & 168.7 & 168.6 & 168.8 & 168.5 & 168.4 & 168.8 & 168.6 & 168.8 & 166.8 & 166.7 \\
\hline 1" & 119.7 & 121.0 & 121.0 & 122.3 & 119.7 & 122.3 & 120.9 & 120.9 & 123.5 & 123.5 & 122.5 & 122.4 & 122.5 & 122.5 & 121.2 & 120.3 & 115.4 \\
\hline $2^{\prime \prime}$ & 121.7 & 120.5 & 120.5 & 120.9 & 121.7 & 120.9 & 121.5 & 121.4 & 121.0 & 121.0 & 120.4 & 120.4 & 120.6 & 120.5 & 121.0 & 120.1 & 123.9 \\
\hline 3" & 112.6 & 111.5 & 111.5 & 111.2 & 112.6 & 111.2 & 112.6 & 112.6 & 111.4 & 111.4 & 111.8 & 111.6 & 111.6 & 111.5 & 111.8 & 111.1 & 108.4 \\
\hline 4" & 145.7 & 146.5 & 146.4 & 146.7 & 145.7 & 146.7 & 145.5 & 145.5 & 146.4 & 146.4 & 146.0 & 146.0 & 146.6 & 146.5 & 146.2 & 144.7 & 146.1 \\
\hline $5^{\prime \prime}$ & 137.4 & 137.4 & 137.3 & 138.0 & 137.4 & 138.1 & 137.5 & 137.5 & 137.9 & 137.9 & 137.3 & 137.3 & 137.8 & 137.8 & 137.6 & 136.3 & 136.2 \\
\hline $6^{\prime \prime}$ & 141.2 & 141.7 & 141.7 & 141.7 & 141.2 & 141.7 & 140.8 & 140.8 & 141.6 & 141.6 & 141.5 & 141.5 & 142.0 & 142.1 & 141.5 & 140.1 & 142.6 \\
\hline $7^{\prime \prime}$ & 176.1 & 172.5 & 172.5 & 172.5 & 176.1 & 172.5 & 176.2 & 176.2 & 172.4 & 172.4 & 172.1 & 172.2 & 172.2 & 172.2 & 173.8 & 171.7 & 169.4 \\
\hline $1^{\prime \prime \prime}$ & 44.3 & 49.1 & 49.1 & 46.5 & 44.4 & 46.5 & 45.0 & 45.1 & 47.1 & 47.1 & 49.6 & 49.5 & 50.0 & 50.0 & 46.8 & 47.6 & 46.4 \\
\hline $2^{\prime \prime \prime}$ & 49.4 & 49.0 & 49.0 & 46.3 & 49.4 & 46.3 & 49.3 & 49.3 & 45.9 & 45.9 & 48.8 & 48.8 & 46.1 & 46.2 & 48.2 & 49.0 & 48.3 \\
\hline $3^{\prime \prime \prime}$ & 34.2 & 34.8 & 34.7 & 33.4 & 34.2 & 33.4 & 34.6 & 34.6 & 34.4 & 34.4 & 35.5 & 35.5 & 34.8 & 34.8 & 34.3 & 35.4 & 37.8 \\
\hline 4"' & 111.3 & 113.3 & 113.3 & 111.6 & 111.2 & 111.7 & 110.4 & 110.4 & 110.5 & 110.5 & 111.6 & 111.7 & 111.0 & 111.0 & 111.8 & 111.1 & 108.2 \\
\hline $5^{\prime \prime \prime}$ & 75.3 & 77.1 & 77.1 & 76.0 & 75.3 & 76.0 & 78.4 & 78.4 & 78.3 & 78.3 & 78.9 & 79.0 & 78.9 & 78.9 & 76.7 & 76.8 & 80.1 \\
\hline $6^{\prime \prime \prime}$ & 177.2 & 176.1 & 176.0 & 176.9 & 177.2 & 176.9 & 171.9 & 171.9 & 172.1 & 172.1 & 171.3 & 171.3 & 173.1 & 173.1 & 175.7 & 173.5 & 172.7 \\
\hline $7^{\prime \prime \prime}$ & 178.2 & 183.5 & 183.5 & 176.2 & 178.1 & 176.2 & 178.3 & 178.3 & 176.3 & 176.3 & 183.7 & 183.8 & 177.1 & 177.1 & 179.3 & 177.1 & 173.7 \\
\hline
\end{tabular}

at $298 \mathrm{~K}$ based on relative Gibbs free energies. ${ }^{d}$ Linearly corrected for the experimental data. 
Table S38. Calculated ${ }^{13} \mathrm{C}$ NMR chemical shifts of $\mathbf{1 F}$.

\begin{tabular}{|c|c|c|c|c|c|c|c|c|c|c|c|c|c|c|c|c|c|c|c|}
\hline \multirow[b]{2}{*}{ Position } & \multicolumn{18}{|c|}{ calculated $^{a}$} & \multirow{2}{*}{$\begin{array}{c}\text { experimental }^{b} \\
1\end{array}$} \\
\hline & $1 F-1$ & $1 F-2$ & $1 F-3$ & $1 F-4$ & $1 F-5$ & $1 F-6$ & 1F-7 & $1 F-8$ & 1F-9 & $1 F-10$ & 1F-11 & 1F-12 & 1F-13 & $1 F-14$ & 1F-15 & 1F-16 & averaged-1 $1 \mathrm{~F}^{c}$ & $\begin{array}{l}\text { averaged-1F } \\
\text { (corrected) }^{d}\end{array}$ & \\
\hline 1 & 91.5 & 91.4 & 91.5 & 91.5 & 91.5 & 91.5 & 91.5 & 91.4 & 91.5 & 91.4 & 91.5 & 91.4 & 91.6 & 92.3 & 91.5 & 93.2 & 91.5 & 91.3 & 95.6 \\
\hline 2 & 71.0 & 71.0 & 71.0 & 71.0 & 71.0 & 70.9 & 71.0 & 71.0 & 71.0 & 71.0 & 71.1 & 71.0 & 71.0 & 70.9 & 71.0 & 71.6 & 71.0 & 71.2 & 74.1 \\
\hline 3 & 75.3 & 75.4 & 75.4 & 75.4 & 75.3 & 75.4 & 75.4 & 75.4 & 75.4 & 75.4 & 75.4 & 75.4 & 75.4 & 72.5 & 75.4 & 74.6 & 75.3 & 75.4 & 75.9 \\
\hline 4 & 85.4 & 85.4 & 85.2 & 85.3 & 84.9 & 84.9 & 85.4 & 85.4 & 84.9 & 84.9 & 85.3 & 85.3 & 84.9 & 88.0 & 84.8 & 74.7 & 85.1 & 85.0 & 75.4 \\
\hline 5 & 70.5 & 70.5 & 70.4 & 70.4 & 70.5 & 70.5 & 70.5 & 70.5 & 70.5 & 70.5 & 70.4 & 70.4 & 70.5 & 71.1 & 70.5 & 68.9 & 70.5 & 70.7 & 71.2 \\
\hline 6 & 64.1 & 64.1 & 64.2 & 64.2 & 64.0 & 64.0 & 64.2 & 64.2 & 63.9 & 63.9 & 64.1 & 64.1 & 64.1 & 66.8 & 63.9 & 63.1 & 64.1 & 64.5 & 66.5 \\
\hline $1^{\prime}$ & 120.2 & 120.1 & 120.2 & 120.1 & 120.2 & 120.0 & 120.2 & 120.1 & 120.1 & 120.0 & 120.2 & 120.1 & 120.2 & 120.2 & 120.2 & 120.1 & 120.1 & 119.3 & 120.4 \\
\hline $2^{\prime}, 6^{\prime}$ & 109.8 & 109.8 & 109.8 & 109.8 & 109.9 & 109.8 & 109.9 & 109.8 & 109.9 & 109.8 & 109.8 & 109.8 & 109.9 & 109.5 & 109.9 & 109.6 & 109.8 & 109.2 & 110.5 \\
\hline $3^{\prime}, 5^{\prime}$ & 145.2 & 145.2 & 145.2 & 145.2 & 145.2 & 145.3 & 145.2 & 145.2 & 145.2 & 145.3 & 145.2 & 145.2 & 145.2 & 145.5 & 145.2 & 144.9 & 145.2 & 143.8 & 146.5 \\
\hline $4^{\prime}$ & 139.3 & 139.4 & 139.2 & 139.4 & 139.4 & 139.4 & 139.3 & 139.4 & 139.4 & 139.4 & 139.2 & 139.4 & 139.3 & 138.8 & 139.3 & 138.9 & 139.3 & 138.0 & 140.4 \\
\hline $7^{\prime}$ & 168.3 & 168.5 & 168.3 & 168.4 & 168.3 & 168.4 & 168.3 & 168.4 & 168.3 & 168.4 & 168.3 & 168.4 & 168.3 & 168.7 & 168.3 & 168.5 & 168.4 & 166.4 & 166.7 \\
\hline 1" & 116.1 & 116.1 & 121.5 & 121.5 & 121.5 & 121.5 & 116.9 & 117.0 & 121.0 & 121.0 & 120.8 & 120.8 & 121.9 & 120.9 & 121.2 & 113.4 & 119.0 & 118.2 & 115.4 \\
\hline 2" & 123.5 & 123.5 & 122.2 & 122.2 & 122.0 & 122.0 & 123.2 & 123.2 & 122.1 & 122.1 & 122.6 & 122.6 & 121.9 & 121.8 & 121.9 & 127.1 & 122.8 & 121.8 & 123.9 \\
\hline 3" & 112.4 & 112.4 & 112.9 & 112.9 & 111.2 & 111.2 & 111.9 & 111.9 & 111.2 & 111.2 & 113.6 & 113.6 & 111.1 & 112.2 & 111.0 & 107.2 & 112.2 & 111.5 & 108.4 \\
\hline 4" & 146.5 & 146.5 & 145.6 & 145.6 & 145.4 & 145.4 & 146.4 & 146.4 & 145.4 & 145.4 & 145.9 & 145.9 & 145.3 & 146.1 & 145.3 & 147.4 & 146.0 & 144.6 & 146.1 \\
\hline 5" & 137.5 & 137.5 & 136.7 & 136.7 & 136.1 & 136.1 & 136.9 & 136.9 & 136.0 & 136.0 & 137.2 & 137.2 & 136.0 & 136.9 & 135.7 & 134.3 & 136.8 & 135.6 & 136.2 \\
\hline 6" & 142.3 & 142.3 & 140.2 & 140.1 & 142.3 & 142.3 & 142.6 & 142.5 & 142.4 & 142.4 & 139.8 & 139.7 & 142.0 & 142.8 & 141.8 & 141.9 & 141.7 & 140.3 & 142.6 \\
\hline 7" & 175.8 & 175.8 & 176.2 & 176.2 & 176.4 & 176.4 & 176.0 & 175.9 & 176.5 & 176.5 & 176.1 & 176.1 & 176.5 & 172.7 & 176.6 & 172.6 & 176.0 & 173.9 & 169.4 \\
\hline 1"' & 47.2 & 47.2 & 47.8 & 47.7 & 46.6 & 46.6 & 47.7 & 47.7 & 47.9 & 47.9 & 47.7 & 47.6 & 46.4 & 46.2 & 46.2 & 47.4 & 47.3 & 48.1 & 46.4 \\
\hline $2^{\prime \prime \prime}$ & 49.4 & 49.5 & 50.1 & 50.1 & 48.7 & 48.7 & 49.9 & 49.9 & 49.3 & 49.3 & 49.3 & 49.3 & 48.7 & 45.3 & 49.3 & 50.5 & 49.5 & 50.2 & 48.3 \\
\hline 3"' & 34.4 & 34.5 & 35.9 & 35.9 & 34.2 & 34.2 & 37.4 & 37.4 & 33.8 & 33.8 & 33.5 & 33.5 & 37.1 & 32.4 & 36.7 & 33.9 & 35.1 & 36.1 & 37.8 \\
\hline 4"' & 110.0 & 110.0 & 109.9 & 109.9 & 109.1 & 109.1 & 109.8 & 109.8 & 109.6 & 109.6 & 109.9 & 109.9 & 108.6 & 107.4 & 108.7 & 110.5 & 109.7 & 109.1 & 108.2 \\
\hline 5"' & 78.9 & 78.9 & 78.7 & 78.7 & 78.1 & 78.1 & 78.1 & 78.1 & 79.6 & 79.6 & 79.5 & 79.5 & 77.3 & 74.6 & 79.0 & 79.6 & 78.6 & 78.7 & 80.1 \\
\hline 6"' & 176.3 & 176.2 & 176.8 & 176.7 & 179.2 & 179.2 & 177.1 & 177.1 & 177.0 & 177.0 & 175.5 & 175.5 & 178.9 & 174.7 & 177.9 & 175.9 & 177.0 & 174.9 & 172.7 \\
\hline 7"' & 176.6 & 176.6 & 176.9 & 176.9 & 177.4 & 177.4 & 176.6 & 176.6 & 177.1 & 177.1 & 176.9 & 176.9 & 177.5 & 175.6 & 177.0 & 176.1 & 176.9 & 174.7 & 173.7 \\
\hline
\end{tabular}

${ }^{a}$ Calculated using the GIAO method at the mPWIPW91/6-311+G(2d,p) level in MeOH $(P C M) .{ }^{b}$ Measured in $\mathrm{CD}{ }_{3} \mathrm{OD}(125 \mathrm{MHz}) .{ }^{c}$ Averaged according to the Boltzmann distribution theory at $298 \mathrm{~K}$ based on

relative Gibbs free energies. 'Linearly corrected for the experimental data. 
Table S39. Calculated ${ }^{13} \mathrm{C}$ NMR chemical shifts of $\mathbf{1 G}$.

\begin{tabular}{|c|c|c|c|c|c|c|c|c|c|c|c|c|}
\hline \multirow[b]{2}{*}{ Position } & \multicolumn{11}{|c|}{ calculated $^{a}$} & \multirow{2}{*}{$\begin{array}{c}\text { experimental }^{b} \\
1\end{array}$} \\
\hline & 1G-1 & 1G-2 & 1G-3 & 1G-4 & 1G-5 & 1G-6 & 1G-7 & 1G-8 & 1G-9 & averaged $-\mathbf{1} G^{c}$ & $\begin{array}{l}\text { averaged-1G } \\
{\text { (corrected })^{d}}^{\text {and }}\end{array}$ & \\
\hline 1 & 91.5 & 91.5 & 91.5 & 91.4 & 91.5 & 91.5 & 92.5 & 91.5 & 91.5 & 91.5 & 91.8 & 95.6 \\
\hline 2 & 71.9 & 71.8 & 71.8 & 71.9 & 71.8 & 71.8 & 71.8 & 71.8 & 71.8 & 71.8 & 72.7 & 74.1 \\
\hline 3 & 75.8 & 75.9 & 75.8 & 75.8 & 75.9 & 75.9 & 73.7 & 75.9 & 75.9 & 75.8 & 76.5 & 75.9 \\
\hline 4 & 82.4 & 82.4 & 82.4 & 82.2 & 82.5 & 82.3 & 74.7 & 82.3 & 82.4 & 82.2 & 82.8 & 75.4 \\
\hline 5 & 69.2 & 69.3 & 69.1 & 69.2 & 69.1 & 69.2 & 69.1 & 69.1 & 69.1 & 69.2 & 70.1 & 71.2 \\
\hline 6 & 62.2 & 62.3 & 62.2 & 62.3 & 62.2 & 62.3 & 62.0 & 62.2 & 62.2 & 62.2 & 63.4 & 66.5 \\
\hline $1^{\prime}$ & 120.4 & 120.3 & 120.4 & 120.4 & 120.3 & 120.3 & 120.8 & 120.4 & 120.3 & 120.4 & 119.8 & 120.4 \\
\hline $2^{\prime}, 6^{\prime}$ & 109.6 & 109.5 & 109.5 & 109.6 & 109.5 & 109.5 & 109.0 & 109.5 & 109.5 & 109.5 & 109.3 & 110.5 \\
\hline $3^{\prime}, 5^{\prime}$ & 145.1 & 145.0 & 145.1 & 145.1 & 145.1 & 145.0 & 144.7 & 145.1 & 145.1 & 145.1 & 143.8 & 146.5 \\
\hline $4^{\prime}$ & 138.9 & 139.2 & 138.9 & 138.8 & 139.2 & 139.1 & 138.3 & 138.9 & 139.2 & 139.0 & 137.9 & 140.4 \\
\hline $7^{\prime}$ & 169.1 & 169.1 & 169.1 & 169.1 & 169.2 & 169.1 & 169.7 & 169.1 & 169.2 & 169.1 & 167.1 & 166.7 \\
\hline 1" & 125.5 & 125.5 & 124.8 & 127.1 & 124.8 & 127.0 & 124.5 & 125.7 & 125.6 & 125.6 & 124.9 & 115.4 \\
\hline 2" & 115.9 & 116.0 & 115.7 & 115.8 & 115.7 & 115.9 & 118.7 & 115.7 & 115.8 & 115.9 & 115.5 & 123.9 \\
\hline $3^{\prime \prime}$ & 114.9 & 114.9 & 114.6 & 115.0 & 114.6 & 115.0 & 114.0 & 114.7 & 114.7 & 114.9 & 114.4 & 108.4 \\
\hline 4" & 144.8 & 144.8 & 144.8 & 144.5 & 144.8 & 144.5 & 145.2 & 144.7 & 144.7 & 144.7 & 143.4 & 146.1 \\
\hline $5^{\prime \prime}$ & 139.1 & 139.1 & 139.1 & 139.2 & 139.1 & 139.3 & 137.4 & 139.2 & 139.2 & 139.1 & 138.0 & 136.2 \\
\hline 6" & 141.5 & 141.5 & 141.9 & 140.9 & 141.8 & 140.9 & 141.0 & 141.5 & 141.5 & 141.5 & 140.3 & 142.6 \\
\hline 7" & 174.2 & 174.2 & 174.1 & 174.3 & 174.1 & 174.3 & 170.5 & 174.1 & 174.1 & 174.1 & 172.0 & 169.4 \\
\hline $1^{\prime \prime \prime}$ & 45.9 & 46.0 & 48.7 & 46.8 & 48.8 & 46.9 & 45.6 & 49.7 & 49.8 & 46.5 & 48.1 & 46.4 \\
\hline 2"' & 46.6 & 46.6 & 46.6 & 46.3 & 46.6 & 46.3 & 47.0 & 46.2 & 46.2 & 46.5 & 48.2 & 48.3 \\
\hline 3"' & 34.1 & 34.0 & 34.4 & 34.5 & 34.3 & 34.5 & 34.0 & 35.1 & 35.0 & 34.2 & 36.2 & 37.8 \\
\hline 4"' & 111.3 & 111.3 & 111.7 & 110.2 & 111.7 & 110.2 & 111.3 & 110.6 & 110.6 & 111.2 & 110.9 & 108.2 \\
\hline $5 " '$ & 75.9 & 75.9 & 76.9 & 78.6 & 76.8 & 78.5 & 75.7 & 79.0 & 79.0 & 76.4 & 77.2 & 80.1 \\
\hline 6"' & 177.4 & 177.5 & 176.2 & 172.2 & 176.2 & 172.2 & 177.6 & 173.5 & 173.5 & 176.5 & 174.3 & 172.7 \\
\hline 7"' & 176.1 & 176.1 & 176.5 & 176.0 & 176.6 & 176.0 & 175.9 & 176.5 & 176.6 & 176.1 & 173.9 & 173.7 \\
\hline
\end{tabular}

according to the Boltzmann distribution theory at $298 \mathrm{~K}$ based on relative Gibbs free energies. ${ }^{d}$ Linearly corrected for the experimental data. 
Table S40. Calculated ${ }^{13} \mathrm{C}$ NMR chemical shifts of $\mathbf{1 H}$.

\begin{tabular}{|c|c|c|c|c|c|c|c|c|c|c|c|c|c|c|c|c|c|c|c|c|c|}
\hline \multirow[b]{2}{*}{ Position } & \multicolumn{20}{|c|}{ calculated $^{a}$} & \multirow{2}{*}{$\begin{array}{c}\text { experimental }^{b} \\
1\end{array}$} \\
\hline & $1 \mathrm{H}-1$ & $1 \mathrm{H}-2$ & $1 \mathrm{H}-3$ & $1 \mathrm{H}-4$ & $1 \mathrm{H}-5$ & $1 \mathrm{H}-6$ & $1 \mathrm{H}-7$ & $1 \mathrm{H}-8$ & 1H-9 & $1 \mathrm{H}-10$ & $1 \mathrm{H}-11$ & $1 \mathrm{H}-12$ & $1 \mathrm{H}-13$ & $1 \mathrm{H}-14$ & 1H-15 & $1 \mathrm{H}-16$ & 1H-17 & $1 \mathrm{H}-18$ & averaged-1 $\mathbf{H}^{c}$ & $\begin{array}{c}\text { averaged-1H } \\
\text { (corrected) }^{d}\end{array}$ & \\
\hline 1 & 91.3 & 91.3 & 91.3 & 91.5 & 91.3 & 91.3 & 91.3 & 91.4 & 91.4 & 91.4 & 91.5 & 91.4 & 91.4 & 91.3 & 91.3 & 91.5 & 91.4 & 91.4 & 91.3 & 91.5 & 95.6 \\
\hline 2 & 72.1 & 72.1 & 72.1 & 71.7 & 72.1 & 72.1 & 72.1 & 72.0 & 72.0 & 71.7 & 71.7 & 71.8 & 71.8 & 72.0 & 72.1 & 71.8 & 71.7 & 71.8 & 72.0 & 72.7 & 74.1 \\
\hline 3 & 75.4 & 75.3 & 75.3 & 75.9 & 75.3 & 75.3 & 75.3 & 75.5 & 75.4 & 75.9 & 75.8 & 75.8 & 75.8 & 75.5 & 75.4 & 75.8 & 75.8 & 75.7 & 75.5 & 76.1 & 75.9 \\
\hline 4 & 82.5 & 81.8 & 82.5 & 82.5 & 81.9 & 82.0 & 82.0 & 82.4 & 82.0 & 82.4 & 82.2 & 82.3 & 82.2 & 82.4 & 82.0 & 82.4 & 82.2 & 82.3 & 82.2 & 82.7 & 75.4 \\
\hline 5 & 69.3 & 69.3 & 69.3 & 69.2 & 69.3 & 69.3 & 69.3 & 69.5 & 69.5 & 69.3 & 68.8 & 69.2 & 68.8 & 69.4 & 69.5 & 69.3 & 67.4 & 69.2 & 69.3 & 70.1 & 71.2 \\
\hline 6 & 62.4 & 62.4 & 62.4 & 62.7 & 62.4 & 62.6 & 62.6 & 62.7 & 62.7 & 62.7 & 62.2 & 62.7 & 62.2 & 62.7 & 62.6 & 62.8 & 65.3 & 62.8 & 62.5 & 63.5 & 66.5 \\
\hline $1^{\prime}$ & 120.8 & 120.9 & 120.7 & 120.3 & 120.8 & 120.9 & 120.7 & 120.7 & 120.8 & 120.3 & 120.5 & 120.5 & 120.5 & 120.7 & 120.8 & 120.4 & 120.9 & 120.5 & 120.7 & 120.1 & 120.4 \\
\hline $2^{\prime}, 6^{\prime}$ & 109.2 & 109.1 & 109.3 & 109.5 & 109.2 & 109.2 & 109.3 & 109.3 & 109.2 & 109.5 & 109.4 & 109.5 & 109.4 & 109.3 & 109.3 & 109.5 & 109.1 & 109.5 & 109.3 & 109.0 & 110.5 \\
\hline $3^{\prime}, 5^{\prime}$ & 144.7 & 144.6 & 144.6 & 145.0 & 144.5 & 144.7 & 144.6 & 144.8 & 144.7 & 145.0 & 145.0 & 145.0 & 144.9 & 144.7 & 144.6 & 145.0 & 144.8 & 144.9 & 144.7 & 143.5 & 146.5 \\
\hline $4^{\prime}$ & 138.2 & 138.0 & 138.0 & 138.9 & 137.8 & 138.1 & 137.8 & 138.3 & 138.2 & 138.9 & 138.7 & 138.5 & 138.4 & 138.0 & 137.9 & 138.8 & 138.5 & 138.4 & 138.2 & 137.1 & 140.4 \\
\hline $7^{\prime}$ & 169.5 & 169.6 & 169.4 & 169.1 & 169.5 & 169.6 & 169.4 & 169.5 & 169.5 & 169.1 & 169.4 & 169.1 & 169.2 & 169.3 & 169.4 & 169.2 & 169.5 & 169.1 & 169.4 & 167.5 & 166.7 \\
\hline $1 "$ & 121.9 & 128.4 & 121.9 & 124.8 & 128.4 & 128.8 & 128.8 & 122.9 & 128.1 & 124.8 & 125.2 & 124.8 & 125.2 & 122.9 & 128.1 & 125.8 & 127.0 & 125.8 & 125.5 & 124.7 & 115.4 \\
\hline 2" & 117.6 & 115.3 & 117.6 & 116.8 & 115.3 & 115.6 & 115.6 & 117.5 & 115.4 & 116.7 & 116.1 & 116.6 & 116.1 & 117.5 & 115.4 & 116.4 & 115.5 & 116.3 & 116.4 & 115.9 & 123.9 \\
\hline $3^{\prime \prime}$ & 115.4 & 113.3 & 115.4 & 114.7 & 113.3 & 114.6 & 114.6 & 115.7 & 113.2 & 114.4 & 113.6 & 114.4 & 113.6 & 115.7 & 113.2 & 114.4 & 113.2 & 114.4 & 114.5 & 114.0 & 108.4 \\
\hline 4" & 145.8 & 144.7 & 145.8 & 145.0 & 144.7 & 144.8 & 144.8 & 145.3 & 144.6 & 145.0 & 144.7 & 145.0 & 144.7 & 145.3 & 144.6 & 144.8 & 145.0 & 144.8 & 145.1 & 143.9 & 146.1 \\
\hline $5^{\prime \prime}$ & 139.1 & 138.8 & 139.1 & 138.7 & 138.8 & 139.0 & 139.0 & 139.1 & 138.5 & 138.6 & 138.6 & 138.6 & 138.6 & 139.1 & 138.5 & 138.8 & 138.4 & 138.7 & 138.9 & 137.8 & 136.2 \\
\hline 6" & 144.2 & 144.5 & 144.3 & 142.3 & 144.5 & 142.7 & 142.7 & 143.0 & 143.9 & 142.8 & 143.4 & 142.7 & 143.5 & 143.1 & 143.9 & 142.3 & 143.6 & 142.2 & 143.6 & 142.4 & 142.6 \\
\hline $7^{\prime \prime}$ & 174.4 & 175.1 & 174.4 & 174.0 & 175.1 & 174.9 & 174.9 & 174.5 & 175.1 & 174.2 & 174.3 & 174.2 & 174.3 & 174.5 & 175.1 & 174.3 & 173.9 & 174.3 & 174.6 & 172.5 & 169.4 \\
\hline $1^{\prime \prime \prime}$ & 48.6 & 47.6 & 48.6 & 44.9 & 47.6 & 48.7 & 48.7 & 45.3 & 45.5 & 45.5 & 48.1 & 45.5 & 48.1 & 45.3 & 45.6 & 44.6 & 47.0 & 44.5 & 47.4 & 48.8 & 46.4 \\
\hline $2^{\prime \prime \prime}$ & 47.3 & 46.9 & 47.3 & 45.1 & 46.9 & 47.3 & 47.3 & 46.1 & 46.4 & 45.5 & 45.5 & 45.5 & 45.5 & 46.1 & 46.4 & 45.3 & 46.7 & 45.3 & 46.6 & 48.1 & 48.3 \\
\hline $3^{\prime \prime \prime}$ & 34.0 & 33.5 & 34.0 & 32.7 & 33.5 & 35.7 & 35.7 & 32.4 & 35.6 & 35.6 & 33.5 & 35.6 & 33.6 & 32.4 & 35.6 & 35.0 & 36.4 & 35.0 & 34.1 & 35.8 & 37.8 \\
\hline 4"' & 110.9 & 109.9 & 110.9 & 106.9 & 109.9 & 110.7 & 110.7 & 109.6 & 108.6 & 106.7 & 108.8 & 106.7 & 108.8 & 109.6 & 108.6 & 106.5 & 108.2 & 106.5 & 109.6 & 109.3 & 108.2 \\
\hline $5^{\prime \prime \prime}$ & 79.1 & 78.5 & 79.1 & 74.7 & 78.5 & 79.2 & 79.2 & 77.8 & 78.5 & 75.3 & 76.8 & 75.4 & 76.8 & 77.9 & 78.6 & 75.8 & 80.4 & 75.8 & 78.0 & 78.6 & 80.1 \\
\hline 6"' & 174.6 & 179.0 & 174.5 & 175.0 & 179.0 & 175.5 & 175.5 & 173.5 & 176.3 & 174.6 & 173.8 & 174.6 & 173.8 & 173.5 & 176.3 & 173.9 & 178.3 & 173.9 & 175.8 & 173.7 & 172.7 \\
\hline $7^{\prime \prime \prime}$ & 174.4 & 175.3 & 174.4 & 175.8 & 175.3 & 174.4 & 174.3 & 175.3 & 175.1 & 175.6 & 176.2 & 175.6 & 176.2 & 175.3 & 175.1 & 175.6 & 185.3 & 175.5 & 175.2 & 173.1 & 173.7 \\
\hline
\end{tabular}

free energies. ${ }^{d}$ Linearly corrected for the experimental data. 
Table S41. Calculated ${ }^{13} \mathrm{C}$ NMR chemical shifts of $\mathbf{1}^{\prime} \mathbf{A}$.

\begin{tabular}{|c|c|c|c|c|c|c|c|c|c|}
\hline \multirow[b]{2}{*}{ Position } & \multicolumn{8}{|c|}{ calculated $^{a}$} & \multirow{2}{*}{$\begin{array}{c}\text { experimental } \\
1\end{array}$} \\
\hline & 1'A-1 & $1^{\prime} \mathrm{A}-2$ & I'A-3 $^{\prime}$ & $1^{\prime} \mathrm{A}-4$ & 1'A-5 & 1'A-6 & $\begin{array}{c}\text { averaged- } \\
\mathbf{1}^{\prime} \mathbf{A}^{c} \\
\end{array}$ & $\begin{array}{l}\text { averaged-1'A } \\
\text { (corrected) }^{d}\end{array}$ & \\
\hline 1 & 94.3 & 93.7 & 93.7 & 101.1 & 101.2 & 101.5 & 95.2 & 93.8 & 95.6 \\
\hline 2 & 73.9 & 73.6 & 73.7 & 76.9 & 76.9 & 77.8 & 74.3 & 73.4 & 74.1 \\
\hline 3 & 71.8 & 74.7 & 74.8 & 74.7 & 74.6 & 75.5 & 73.4 & 72.6 & 75.9 \\
\hline 4 & 70.7 & 70.8 & 70.8 & 69.6 & 69.6 & 70.8 & 70.6 & 69.8 & 75.4 \\
\hline 5 & 71.6 & 71.5 & 71.5 & 72.4 & 72.4 & 71.8 & 71.7 & 70.9 & 71.2 \\
\hline 6 & 66.8 & 66.7 & 66.7 & 66.7 & 66.8 & 66.7 & 66.8 & 66.1 & 66.5 \\
\hline $1^{\prime}$ & 119.6 & 119.8 & 119.8 & 119.8 & 119.5 & 119.6 & 119.7 & 117.6 & 120.4 \\
\hline $2^{\prime}, 6^{\prime}$ & 109.8 & 109.8 & 109.9 & 109.7 & 110.0 & 109.9 & 109.8 & 108.0 & 110.5 \\
\hline $3^{\prime}, 5^{\prime}$ & 145.2 & 145.2 & 145.0 & 145.3 & 145.5 & 145.3 & 145.2 & 142.5 & 146.5 \\
\hline $4^{\prime}$ & 139.9 & 139.7 & 139.5 & 139.8 & 139.9 & 139.8 & 139.8 & 137.2 & 140.4 \\
\hline $7^{\prime}$ & 168.5 & 168.3 & 168.3 & 172.8 & 172.5 & 172.5 & 169.1 & 165.7 & 166.7 \\
\hline 1" & 119.2 & 118.6 & 118.6 & 118.9 & 118.9 & 118.4 & 118.9 & 116.9 & 115.4 \\
\hline 2" & 121.2 & 121.1 & 121.1 & 121.2 & 121.2 & 121.2 & 121.2 & 119.1 & 123.9 \\
\hline $3^{\prime \prime}$ & 106.6 & 106.5 & 106.5 & 106.6 & 106.6 & 106.4 & 106.5 & 104.8 & 108.4 \\
\hline 4" & 147.6 & 147.7 & 147.7 & 147.6 & 147.6 & 147.6 & 147.7 & 144.9 & 146.1 \\
\hline 5" & 131.3 & 131.3 & 131.3 & 131.1 & 131.1 & 130.9 & 131.2 & 128.9 & 136.2 \\
\hline 6" & 147.3 & 147.3 & 147.3 & 147.3 & 147.2 & 147.2 & 147.3 & 144.5 & 142.6 \\
\hline 7" & 173.9 & 174.5 & 174.5 & 173.9 & 173.9 & 174.4 & 174.1 & 170.6 & 169.4 \\
\hline 1"' & 59.2 & 59.1 & 59.1 & 59.2 & 59.2 & 59.0 & 59.2 & 58.7 & 46.4 \\
\hline $2^{\prime \prime \prime}$ & 48.3 & 48.3 & 48.4 & 48.3 & 48.3 & 48.3 & 48.3 & 48.1 & 48.3 \\
\hline $3^{\prime \prime \prime}$ & 37.1 & 37.2 & 37.2 & 37.2 & 37.2 & 37.4 & 37.2 & 37.3 & 37.8 \\
\hline $4^{\prime \prime \prime}$ & 123.3 & 123.3 & 123.3 & 123.5 & 123.4 & 123.6 & 123.3 & 121.1 & 108.2 \\
\hline 5"' & 80.7 & 80.6 & 80.6 & 80.9 & 80.8 & 80.9 & 80.7 & 79.7 & 80.1 \\
\hline 6"' & 181.9 & 181.9 & 181.9 & 181.9 & 181.9 & 181.9 & 181.9 & 178.2 & 172.7 \\
\hline 7"' & 185.6 & 185.4 & 185.4 & 185.7 & 185.7 & 185.5 & 185.5 & 181.7 & 173.7 \\
\hline
\end{tabular}

Calculated using the GIAO method at the mPWIPW91/6-311+G(2d,p) level in MeOH (PCM). ${ }^{b}$ Measured in

$\mathrm{CD}_{3} \mathrm{OD}(125 \mathrm{MHz}) .{ }^{c}$ Averaged according to the Boltzmann distribution theory at $298 \mathrm{~K}$ based on relative Gibbs

free energies. ${ }^{d}$ Linearly corrected for the experimental data. 
Table S42. Calculated ${ }^{13} \mathbf{C}$ NMR chemical shifts of $\mathbf{1}^{\prime} \mathbf{B}$.

\begin{tabular}{|c|c|c|c|c|c|c|c|c|c|c|c|c|c|c|c|}
\hline \multirow[b]{2}{*}{ Position } & \multicolumn{14}{|c|}{ calculated $^{a}$} & \multirow{2}{*}{$\frac{\text { experimental }^{b}}{1}$} \\
\hline & 1'B-1 & 1'B-2 & 1'B-3 & 1 'B-4 & 1'B-5 & 1'B-6 & 1'B-7 & 1'B-8 & 1'B-9 & 'B'B-10 & 1'B-11 & 1'B-12 & $\begin{array}{c}\text { averaged- } \\
\mathbf{1}^{\prime} \mathbf{B}^{c} \\
\end{array}$ & $\begin{array}{l}\text { averaged-1'B }^{\prime} \\
\text { (corrected) }^{d}\end{array}$ & \\
\hline 1 & 93.7 & 101.0 & 101.0 & 92.4 & 92.0 & 92.0 & 92.0 & 92.1 & 92.0 & 91.7 & 92.2 & 91.8 & 95.2 & 94.2 & 95.6 \\
\hline 2 & 73.4 & 76.7 & 76.7 & 73.1 & 72.8 & 72.8 & 72.8 & 70.9 & 73.2 & 70.5 & 70.8 & 70.6 & 74.0 & 73.4 & 74.1 \\
\hline 3 & 74.7 & 74.7 & 74.6 & 68.7 & 68.5 & 68.5 & 68.6 & 71.4 & 68.7 & 71.2 & 71.4 & 71.2 & 72.7 & 72.1 & 75.9 \\
\hline 4 & 71.7 & 70.5 & 70.5 & 77.6 & 78.3 & 78.3 & 78.3 & 79.1 & 77.4 & 80.0 & 79.1 & 79.7 & 73.7 & 73.1 & 75.4 \\
\hline 5 & 71.8 & 72.6 & 72.6 & 65.8 & 64.9 & 64.7 & 64.9 & 66.0 & 66.0 & 64.7 & 66.0 & 65.2 & 69.7 & 69.1 & 71.2 \\
\hline 6 & 66.8 & 66.7 & 66.8 & 62.0 & 63.0 & 63.2 & 63.0 & 62.0 & 62.0 & 63.2 & 62.0 & 62.7 & 65.2 & 64.8 & 66.5 \\
\hline $1^{\prime}$ & 119.9 & 119.8 & 119.4 & 120.2 & 120.5 & 120.6 & 120.2 & 120.2 & 119.8 & 120.8 & 120.4 & 120.4 & 120.0 & 118.4 & 120.4 \\
\hline $2^{\prime}, 6^{\prime}$ & 109.7 & 109.7 & 110.1 & 109.6 & 109.5 & 109.4 & 109.2 & 109.4 & 109.5 & 109.4 & 109.7 & 109.2 & 109.7 & 108.3 & 110.5 \\
\hline $3^{\prime}, 5^{\prime}$ & 145.5 & 145.3 & 145.4 & 144.7 & 144.6 & 144.8 & 144.4 & 144.4 & 144.6 & 144.6 & 144.6 & 144.3 & 145.1 & 143.0 & 146.5 \\
\hline $4^{\prime}$ & 140.0 & 139.9 & 140.0 & 138.2 & 137.7 & 137.8 & 137.8 & 138.1 & 138.7 & 137.5 & 138.0 & 137.7 & 139.2 & 137.2 & 140.4 \\
\hline $7^{\prime}$ & 168.5 & 172.9 & 172.7 & 168.6 & 168.8 & 168.8 & 168.6 & 168.1 & 168.3 & 168.7 & 168.4 & 168.4 & 169.8 & 167.1 & 166.7 \\
\hline 1" & 120.0 & 120.2 & 120.2 & 118.2 & 116.6 & 118.2 & 116.4 & 118.4 & 118.3 & 118.3 & 118.5 & 116.9 & 119.2 & 117.6 & 115.4 \\
\hline 2" & 121.8 & 122.0 & 122.0 & 120.8 & 121.3 & 121.7 & 121.3 & 120.4 & 120.7 & 121.3 & 120.4 & 120.8 & 121.6 & 120.0 & 123.9 \\
\hline 3" & 107.7 & 107.9 & 107.9 & 112.4 & 112.5 & 111.9 & 112.5 & 112.4 & 112.3 & 112.0 & 112.4 & 112.5 & 109.4 & 108.0 & 108.4 \\
\hline 4" & 148.1 & 148.0 & 148.0 & 147.4 & 147.8 & 147.4 & 147.9 & 147.4 & 147.4 & 147.4 & 147.4 & 147.8 & 147.9 & 145.7 & 146.1 \\
\hline 5" & 132.3 & 132.3 & 132.3 & 133.4 & 133.1 & 132.9 & 133.1 & 133.7 & 133.6 & 133.1 & 133.7 & 133.4 & 132.7 & 130.8 & 136.2 \\
\hline 6" & 145.8 & 145.8 & 145.8 & 145.6 & 145.9 & 146.2 & 146.0 & 145.6 & 145.8 & 146.2 & 145.6 & 146.0 & 145.8 & 143.7 & 142.6 \\
\hline 7" & 174.8 & 174.2 & 174.2 & 169.1 & 169.3 & 169.7 & 169.3 & 169.3 & 169.0 & 170.0 & 169.4 & 169.5 & 172.6 & 169.9 & 169.4 \\
\hline $1^{\prime \prime \prime}$ & 57.8 & 58.1 & 58.1 & 58.9 & 59.4 & 58.9 & 59.4 & 58.9 & 59.1 & 58.9 & 58.8 & 59.4 & 58.4 & 58.0 & 46.4 \\
\hline $2^{\prime \prime \prime}$ & 44.6 & 44.8 & 44.8 & 45.8 & 45.8 & 45.3 & 45.9 & 46.0 & 46.0 & 45.4 & 45.8 & 46.2 & 45.1 & 45.0 & 48.3 \\
\hline $3^{\prime \prime \prime}$ & 36.7 & 36.8 & 36.8 & 33.3 & 37.7 & 37.3 & 37.7 & 33.2 & 33.0 & 37.3 & 33.3 & 37.3 & 36.3 & 36.5 & 37.8 \\
\hline 4"' & 120.2 & 120.3 & 120.3 & 118.8 & 118.7 & 120.8 & 118.7 & 118.8 & 118.7 & 120.8 & 118.9 & 118.9 & 119.9 & 118.2 & 108.2 \\
\hline $5^{\prime \prime \prime}$ & 85.7 & 85.5 & 85.5 & 82.8 & 83.6 & 83.4 & 83.6 & 82.9 & 82.8 & 83.3 & 82.9 & 83.7 & 84.7 & 83.9 & 80.1 \\
\hline 6"' & 178.0 & 178.0 & 178.0 & 175.4 & 174.9 & 178.5 & 174.9 & 175.4 & 175.4 & 178.4 & 175.4 & 174.9 & 177.2 & 174.4 & 172.7 \\
\hline 7"' & 186.2 & 186.5 & 186.5 & 177.3 & 177.0 & 177.7 & 177.0 & 177.2 & 177.3 & 177.6 & 177.2 & 176.8 & 183.1 & 180.1 & 173.7 \\
\hline
\end{tabular}

${ }^{a}$ Calculated using the GIAO method at the mPWIPW91/6-311+G(2d,p) level in MeOH (PCM). ${ }^{b}$ Measured in $C D{ }_{3} \mathrm{OD}(125 \mathrm{MHz}) .{ }^{c}$ Averaged according to the Boltzmann

distribution theory at $298 \mathrm{~K}$ based on relative Gibbs free energies. ${ }^{d}$ Linearly corrected for the experimental data. 
Table S43. Calculated ${ }^{13} \mathrm{C}$ NMR chemical shifts of $\mathbf{1}^{\prime} \mathbf{C}$.

\begin{tabular}{|c|c|c|c|c|c|c|c|c|c|c|c|c|c|c|c|c|c|c|c|}
\hline \multirow[b]{2}{*}{ Position } & \multicolumn{18}{|c|}{ calculated $^{a}$} & \multirow{2}{*}{$\begin{array}{c}\text { experimental }^{b} \\
1\end{array}$} \\
\hline & $1^{\prime} \mathrm{C}-1$ & $1^{\prime} \mathrm{C}-2$ & $1^{\prime} \mathrm{C}-3$ & $1^{\prime} \mathrm{C}-4$ & $1^{\prime} \mathrm{C}-5$ & $1^{\prime} \mathrm{C}-6$ & 1'C-7 & $1^{\prime} \mathrm{C}-8$ & 1'C-9 & $1^{\prime} \mathrm{C}-10$ & $1^{\prime} \mathrm{C}-11$ & $1^{\prime} \mathrm{C}-12$ & $1^{\prime} \mathrm{C}-13$ & $1^{\prime} \mathrm{C}-14$ & $1^{\prime} \mathrm{C}-15$ & 1'C-16 & $\begin{array}{c}\text { averaged- } \\
\mathbf{1}^{\prime} \mathbf{C}^{c} \\
\end{array}$ & $\begin{array}{c}\text { averaged-1'C } \\
\text { (corrected) }^{d}\end{array}$ & \\
\hline 1 & 92.6 & 92.7 & 92.6 & 92.6 & 93.1 & 93.2 & 92.5 & 92.5 & 93.2 & 93.1 & 92.6 & 999.8 & 92.5 & 92.7 & 92.7 & 99.8 & 93.0 & 91.4 & 95.6 \\
\hline 2 & 72.8 & 72.8 & 72.9 & 73.0 & 73.2 & 73.1 & 73.1 & 72.9 & 73.2 & 73.2 & 73.0 & 75.4 & 73.1 & 72.7 & 72.7 & 75.4 & 73.0 & 71.7 & 74.1 \\
\hline 3 & 75.6 & 75.7 & 75.7 & 75.8 & 75.7 & 75.7 & 75.7 & 75.6 & 75.6 & 75.6 & 75.8 & 75.4 & 75.7 & 75.6 & 75.7 & 75.4 & 75.7 & 74.3 & 75.9 \\
\hline 4 & 75.5 & 75.4 & 75.8 & 76.2 & 77.0 & 77.0 & 76.2 & 75.8 & 77.3 & 77.3 & 76.2 & 71.9 & 76.2 & 75.4 & 75.3 & 71.9 & 75.8 & 74.4 & 75.4 \\
\hline 5 & 69.8 & 69.8 & 70.0 & 70.5 & 71.2 & 71.2 & 70.4 & 70.0 & 71.4 & 71.4 & 70.4 & 71.3 & 70.4 & 69.7 & 69.7 & 71.3 & 70.2 & 69.0 & 71.2 \\
\hline 6 & 61.4 & 61.4 & 61.5 & 61.6 & 61.8 & 61.8 & 61.6 & 61.5 & 61.9 & 61.9 & 61.6 & 61.5 & 61.6 & 61.3 & 61.3 & 61.5 & 61.5 & 60.4 & 66.5 \\
\hline $1^{\prime}$ & 120.6 & 120.8 & 120.8 & 120.8 & 120.6 & 120.8 & 120.5 & 120.6 & 120.8 & 120.6 & 120.8 & 119.2 & 120.5 & 120.6 & 120.7 & 119.2 & 120.6 & 118.5 & 120.4 \\
\hline $2^{\prime}, 6^{\prime}$ & 127.3 & 126.8 & 126.7 & 126.7 & 126.2 & 127.0 & 126.9 & 127.1 & 127.1 & 126.2 & 126.7 & 127.5 & 126.9 & 127.3 & 126.8 & 127.8 & 126.9 & 124.7 & 110.5 \\
\hline $3^{\prime}, 5^{\prime}$ & 145.1 & 145.1 & 144.9 & 144.7 & 144.2 & 144.4 & 144.6 & 144.8 & 144.4 & 144.2 & 144.7 & 145.2 & 144.6 & 145.2 & 145.1 & 145.3 & 144.9 & 142.4 & 146.5 \\
\hline $4^{\prime}$ & 138.7 & 138.8 & 138.5 & 138.2 & 137.4 & 137.4 & 138.2 & 138.4 & 137.4 & 137.4 & 138.3 & 140.3 & 138.2 & 138.9 & 138.9 & 140.6 & 138.5 & 136.1 & 140.4 \\
\hline $7^{\prime}$ & 168.8 & 168.9 & 169.0 & 169.0 & 168.9 & 169.1 & 168.9 & 168.9 & 169.0 & 168.8 & 169.0 & 172.3 & 168.9 & 168.7 & 168.9 & 172.2 & 169.1 & 166.2 & 166.7 \\
\hline 1" & 118.2 & 118.1 & 116.4 & 117.9 & 119.9 & 119.9 & 117.8 & 116.4 & 120.6 & 120.6 & 118.0 & 116.7 & 118.0 & 118.0 & 117.9 & 116.7 & 118.2 & 116.2 & 115.4 \\
\hline 2" & 119.2 & 119.3 & 119.1 & 118.9 & 118.8 & 118.8 & 118.9 & 119.1 & 118.8 & 118.8 & 119.0 & 121.6 & 119.0 & 119.3 & 119.3 & 121.5 & 119.2 & 117.2 & 123.9 \\
\hline 3" & 115.3 & 115.3 & 115.7 & 115.8 & 115.8 & 115.8 & 115.8 & 115.7 & 115.6 & 115.6 & 115.7 & 113.6 & 115.7 & 115.1 & 115.1 & 113.6 & 115.4 & 113.4 & 108.4 \\
\hline 4" & 146.9 & 146.9 & 147.5 & 147.1 & 147.2 & 147.2 & 147.1 & 147.5 & 147.1 & 147.1 & 147.1 & 147.0 & 147.1 & 146.8 & 146.8 & 147.0 & 147.1 & 144.6 & 146.1 \\
\hline $5^{\prime \prime}$ & 135.1 & 135.0 & 135.4 & 135.4 & 135.5 & 135.5 & 135.4 & 135.5 & 135.4 & 135.4 & 135.4 & 133.2 & 135.4 & 134.6 & 134.6 & 133.2 & 135.1 & 132.8 & 136.2 \\
\hline 6" & 148.0 & 148.0 & 148.0 & 146.7 & 146.9 & 146.9 & 146.7 & 148.0 & 146.8 & 146.8 & 146.9 & 147.1 & 146.9 & 147.6 & 147.6 & 147.1 & 147.5 & 145.0 & 142.6 \\
\hline 7" & 174.1 & 174.1 & 173.5 & 173.5 & 173.7 & 173.7 & 173.6 & 173.6 & 174.0 & 174.0 & 173.6 & 170.8 & 173.6 & 174.1 & 174.1 & 170.8 & 173.7 & 170.8 & 169.4 \\
\hline 1"' & 58.6 & 58.6 & 59.1 & 58.5 & 55.6 & 55.7 & 58.5 & 59.2 & 54.3 & 54.3 & 58.0 & 58.1 & 58.1 & 58.2 & 58.1 & 58.2 & 57.9 & 56.9 & 46.4 \\
\hline $2^{\prime \prime \prime}$ & 46.5 & 46.5 & 46.6 & 46.2 & 45.3 & 45.3 & 46.1 & 46.6 & 45.4 & 45.5 & 46.7 & 45.9 & 46.7 & 45.6 & 45.7 & 45.9 & 46.2 & 45.3 & 48.3 \\
\hline 3"' & 36.5 & 36.5 & 36.9 & 32.4 & 31.9 & 31.9 & 32.4 & 36.9 & 31.6 & 31.6 & 32.4 & 37.3 & 32.4 & 36.3 & 36.4 & 37.3 & 35.1 & 34.3 & 37.8 \\
\hline 4"' & 120.1 & 120.1 & 117.3 & 117.8 & 118.7 & 118.7 & 117.8 & 117.3 & 120.0 & 120.0 & 119.1 & 120.7 & 119.1 & 119.8 & 119.7 & 120.7 & 119.4 & 117.3 & 108.2 \\
\hline $5^{\prime \prime \prime}$ & 83.8 & 83.8 & 83.8 & 83.1 & 80.9 & 80.9 & 83.2 & 83.8 & 79.2 & 79.2 & 82.3 & 83.4 & 82.4 & 86.4 & 86.4 & 83.4 & 83.0 & 81.5 & 80.1 \\
\hline 6"' & 178.4 & 178.4 & 175.2 & 175.3 & 174.2 & 174.2 & 175.3 & 175.2 & 178.7 & 178.7 & 177.0 & 178.3 & 177.0 & 172.9 & 172.9 & 178.3 & 177.1 & 174.1 & 172.7 \\
\hline 7"' & 177.1 & 177.1 & 176.3 & 176.4 & 176.3 & 176.3 & 176.5 & 176.3 & 176.3 & 176.3 & 176.5 & 176.8 & 176.5 & 177.3 & 177.3 & 176.8 & 176.8 & 173.8 & 173.7 \\
\hline
\end{tabular}

${ }^{a}$ Calculated using the GIAO method at the mPWIPW91/6-311+G(2d,p) level in MeOH $(P C M) .{ }^{b}$ Measured in $\mathrm{CD}_{3} \mathrm{OD}(125 \mathrm{MHz}) .{ }^{c}$ Averaged according to the Boltzmann distribution theory at $298 \mathrm{~K}$ based

on relative Gibbs free energies. ${ }^{d}$ Linearly corrected for the experimental data. 
Table S44. Calculated ${ }^{13} \mathrm{C}$ NMR chemical shifts of $\mathbf{1}^{\prime} \mathbf{D}$.

\begin{tabular}{|c|c|c|c|c|c|c|c|c|c|c|c|c|c|}
\hline \multirow[b]{2}{*}{ Position } & \multicolumn{12}{|c|}{ calculated $^{a}$} & \multirow{2}{*}{$\frac{\text { experimental }^{b}}{1}$} \\
\hline & 1'D-1 & 1'D-2 & 1'D-3 & 1'D-4 & 1'D-5 & 1'D-6 & 1'D-7 & 1'D-8 & 1'D-9 & 1'D-10 & $\begin{array}{c}\text { averaged- } \\
\mathbf{1}^{\prime} \mathbf{D}^{c} \\
\end{array}$ & $\begin{array}{l}\text { averaged-1'D } \text { (corrected) }^{d} \\
\text { (c) }\end{array}$ & \\
\hline 1 & 92.6 & 92.7 & 93.5 & 93.0 & 93.0 & 93.0 & 93.8 & 99.8 & 92.6 & 92.6 & 92.9 & 91.8 & 95.6 \\
\hline 2 & 73.0 & 73.0 & 73.1 & 73.1 & 73.0 & 73.0 & 73.2 & 75.7 & 72.7 & 72.7 & 73.0 & 72.2 & 74.1 \\
\hline 3 & 75.8 & 75.9 & 72.8 & 75.9 & 75.8 & 75.9 & 72.7 & 75.5 & 75.7 & 75.7 & 75.6 & 74.7 & 75.9 \\
\hline 4 & 76.1 & 76.1 & 73.3 & 76.4 & 76.3 & 76.3 & 73.2 & 72.0 & 75.4 & 75.4 & 75.8 & 74.9 & 75.4 \\
\hline 5 & 70.2 & 70.3 & 70.8 & 70.6 & 70.6 & 70.6 & 71.0 & 71.7 & 69.7 & 69.7 & 70.3 & 69.5 & 71.2 \\
\hline 6 & 61.6 & 61.7 & 61.9 & 61.8 & 61.7 & 61.8 & 61.9 & 61.7 & 61.4 & 61.4 & 61.7 & 61.0 & 66.5 \\
\hline $1^{\prime}$ & 120.5 & 120.8 & 120.4 & 120.6 & 120.9 & 120.6 & 120.7 & 119.2 & 120.5 & 120.6 & 120.6 & 119.3 & 120.4 \\
\hline $2^{\prime}, 6^{\prime}$ & 109.3 & 109.2 & 109.1 & 109.1 & 109.2 & 109.1 & 109.2 & 110.0 & 109.6 & 109.3 & 109.3 & 108.1 & 110.5 \\
\hline $3^{\prime}, 5^{\prime}$ & 144.4 & 144.6 & 144.5 & 144.2 & 144.4 & 144.2 & 144.8 & 144.9 & 145.2 & 145.1 & 144.5 & 142.9 & 146.5 \\
\hline $4^{\prime}$ & 137.8 & 138.0 & 137.9 & 137.4 & 137.5 & 137.4 & 138.0 & 139.5 & 138.9 & 138.9 & 137.9 & 136.4 & 140.4 \\
\hline $7^{\prime}$ & 169.0 & 169.1 & 169.2 & 169.0 & 169.2 & 169.1 & 169.2 & 172.8 & 168.7 & 168.8 & 169.1 & 167.3 & 166.7 \\
\hline 1" & 118.2 & 118.1 & 117.0 & 119.4 & 119.3 & 119.3 & 118.2 & 117.4 & 118.2 & 118.2 & 118.2 & 116.9 & 115.4 \\
\hline 2" & 119.3 & 119.3 & 121.4 & 119.1 & 119.1 & 119.1 & 121.2 & 121.2 & 119.3 & 119.4 & 119.5 & 118.2 & 123.9 \\
\hline 3" & 116.9 & 116.9 & 115.4 & 116.9 & 116.8 & 116.9 & 115.7 & 115.4 & 115.2 & 115.2 & 116.7 & 115.4 & 108.4 \\
\hline 4" & 147.5 & 147.5 & 147.4 & 147.4 & 147.5 & 147.4 & 147.4 & 147.4 & 147.0 & 147.0 & 147.5 & 145.9 & 146.1 \\
\hline 5" & 135.3 & 135.3 & 133.9 & 135.5 & 135.4 & 135.5 & 134.3 & 134.9 & 134.6 & 134.6 & 135.2 & 133.7 & 136.2 \\
\hline 6" & 145.4 & 145.3 & 144.9 & 146.5 & 146.4 & 146.5 & 146.2 & 145.7 & 147.3 & 147.3 & 145.5 & 143.9 & 142.6 \\
\hline 7" & 173.3 & 173.3 & 170.2 & 173.2 & 173.1 & 173.2 & 170.2 & 170.2 & 174.1 & 174.0 & 173.0 & 171.1 & 169.4 \\
\hline $1^{\prime \prime \prime}$ & 57.3 & 57.2 & 56.5 & 56.8 & 56.9 & 56.8 & 56.2 & 56.6 & 58.3 & 58.3 & 57.1 & 56.5 & 46.4 \\
\hline $2^{\prime \prime \prime}$ & 46.2 & 46.3 & 46.1 & 45.5 & 45.5 & 45.5 & 45.7 & 45.7 & 44.8 & 44.9 & 46.1 & 45.5 & 48.3 \\
\hline 3"' & 36.2 & 36.2 & 36.0 & 36.4 & 36.3 & 36.3 & 36.2 & 36.3 & 35.4 & 35.4 & 36.2 & 35.7 & 37.8 \\
\hline 4"' & 120.1 & 120.1 & 120.1 & 118.7 & 118.8 & 118.7 & 118.9 & 120.0 & 119.8 & 119.8 & 119.9 & 118.6 & 108.2 \\
\hline $5^{\prime \prime \prime}$ & 84.3 & 84.3 & 84.2 & 85.5 & 85.6 & 85.5 & 85.6 & 84.5 & 83.9 & 83.9 & 84.5 & 83.5 & 80.1 \\
\hline 6"' & 176.8 & 176.8 & 176.9 & 176.3 & 176.4 & 176.3 & 176.4 & 177.0 & 178.8 & 178.9 & 176.8 & 174.9 & 172.7 \\
\hline 7"' & 176.4 & 176.4 & 176.2 & 176.2 & 176.2 & 176.2 & 176.1 & 175.9 & 177.5 & 177.4 & 176.4 & 174.5 & 173.7 \\
\hline
\end{tabular}


Table S45. Calculated ${ }^{13} \mathrm{C}$ NMR chemical shifts of $\mathbf{1} \mathbf{E}$.

\begin{tabular}{|c|c|c|c|c|c|c|c|c|c|c|c|c|}
\hline \multirow[b]{2}{*}{ Position } & \multicolumn{11}{|c|}{ calculated $^{a}$} & \multirow{2}{*}{$\begin{array}{c}\text { experimental }^{b} \\
1 \\
\end{array}$} \\
\hline & 1'E-1 & $1^{\prime} \mathrm{E}-2$ & $1^{\prime} \mathrm{E}-3$ & $1^{\prime} \mathrm{E}-4$ & $1^{\prime} \mathrm{E}-5$ & 1'E-6 & 1'E-7 & 1'E-8 & 1'E-9 & $\begin{array}{c}\text { averaged- } \\
\mathbf{1}^{\prime} \mathbf{E}^{c} \\
\end{array}$ & $\begin{array}{l}\text { averaged-1'E } \\
\text { (corrected) }^{d}\end{array}$ & \\
\hline 1 & 92.6 & 92.6 & 92.7 & $\begin{array}{l}92.8 \\
\end{array}$ & 92.6 & 92.5 & 97.0 & 92.7 & $\begin{array}{l}92.8 \\
\end{array}$ & 92.7 & 91.7 & 95.6 \\
\hline 2 & 72.1 & 72.2 & 69.9 & 69.9 & 73.0 & 72.2 & 74.8 & 69.9 & 69.9 & 71.4 & 70.7 & 74.1 \\
\hline 3 & 71.2 & 71.3 & 73.5 & 73.5 & 67.9 & 71.3 & 73.2 & 73.5 & 73.5 & 72.0 & 71.2 & 75.9 \\
\hline 4 & 74.2 & 74.2 & 75.1 & 75.1 & 80.8 & 74.1 & 73.7 & 75.0 & 75.0 & 74.8 & 74.1 & 75.4 \\
\hline 5 & 70.2 & 70.2 & 70.0 & 70.0 & 65.2 & 70.2 & 71.9 & 70.0 & 70.0 & 69.9 & 69.3 & 71.2 \\
\hline 6 & 65.6 & 65.5 & 65.3 & 65.3 & 61.7 & 65.5 & 65.6 & 65.2 & 65.2 & 65.3 & 64.7 & 66.5 \\
\hline $1^{\prime}$ & 120.3 & 120.3 & 120.8 & 120.7 & 120.1 & 120.3 & 119.9 & 120.7 & 120.7 & 120.5 & 119.1 & 120.4 \\
\hline $2^{\prime}, 6^{\prime}$ & 109.3 & 109.4 & 109.1 & 109.3 & 109.8 & 109.5 & 109.7 & 109.2 & 109.3 & 109.3 & 108.1 & 110.5 \\
\hline 3', 5' & 144.3 & 144.5 & 144.2 & 144.4 & 144.9 & 144.4 & 145.4 & 144.2 & 144.4 & 144.4 & 142.6 & 146.5 \\
\hline $4^{\prime}$ & 138.0 & 138.1 & 137.7 & 137.8 & 138.3 & 138.1 & 140.1 & 137.7 & 137.8 & 138.0 & 136.3 & 140.4 \\
\hline $7^{\prime}$ & 168.5 & 168.4 & 168.6 & 168.5 & 168.5 & 168.3 & 172.6 & 168.5 & 168.4 & 168.6 & 166.5 & 166.7 \\
\hline 1" & 122.3 & 122.3 & 122.6 & 122.5 & 122.1 & 122.8 & 122.3 & 123.0 & 123.0 & 122.4 & 121.0 & 115.4 \\
\hline 2" & 121.6 & 121.5 & 121.1 & 121.1 & 119.9 & 121.4 & 121.0 & 121.1 & 121.1 & 121.3 & 119.9 & 123.9 \\
\hline 3" & 108.9 & 108.9 & 108.7 & 108.7 & 112.7 & 108.8 & 108.6 & 108.6 & 108.6 & 109.0 & 107.8 & 108.4 \\
\hline 4" & 147.4 & 147.4 & 147.4 & 147.4 & 147.6 & 147.3 & 147.2 & 147.3 & 147.3 & 147.4 & 145.6 & 146.1 \\
\hline 5" & 132.7 & 132.7 & 132.7 & 132.7 & 133.8 & 132.9 & 132.2 & 133.0 & 133.0 & 132.7 & 131.2 & 136.2 \\
\hline 6" & 145.6 & 145.6 & 145.7 & 145.7 & 146.1 & 146.4 & 145.0 & 146.4 & 146.5 & 145.7 & 144.0 & 142.6 \\
\hline 7" & 173.5 & 173.5 & 173.6 & 173.6 & 170.4 & 173.5 & 173.5 & 173.6 & 173.6 & 173.4 & 171.2 & 169.4 \\
\hline 1"' & 58.7 & 58.7 & 58.8 & 58.8 & 62.2 & 57.5 & 59.0 & 57.6 & 57.6 & 58.8 & 58.3 & 46.4 \\
\hline $2^{2 \prime \prime}$ & 47.6 & 47.6 & 47.6 & 47.6 & 48.0 & 46.5 & 47.8 & 46.4 & 46.5 & 47.5 & 47.2 & 48.3 \\
\hline 3"' & 39.5 & 39.5 & 39.6 & 39.6 & 39.5 & 37.0 & 39.4 & 37.1 & 37.1 & 39.4 & 39.1 & 37.8 \\
\hline $4^{\prime \prime \prime}$ & 122.0 & 122.0 & 122.1 & 122.1 & 121.9 & 121.7 & 121.9 & 121.8 & 121.8 & 122.0 & 120.6 & 108.2 \\
\hline $5^{\prime \prime \prime}$ & 78.6 & 78.6 & 78.6 & 78.6 & 80.0 & 78.2 & 78.7 & 78.2 & 78.2 & 78.6 & 77.8 & 80.1 \\
\hline 6"' & 181.6 & 181.6 & 181.6 & 181.6 & 182.2 & 179.6 & 181.8 & 179.6 & 179.6 & 181.5 & 179.2 & 172.7 \\
\hline 7"' & 177.4 & 177.4 & 177.4 & 177.4 & 180.7 & 178.0 & 177.4 & 178.0 & 178.0 & 177.6 & 175.4 & 173.7 \\
\hline
\end{tabular}

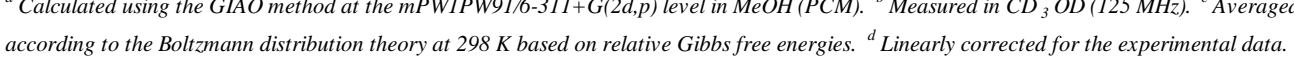


Table S46. Calculated ${ }^{13} \mathrm{C}$ NMR chemical shifts of $\mathbf{1}^{\prime} \mathbf{F}$.

\begin{tabular}{|c|c|c|c|c|c|c|c|c|c|c|c|c|c|c|c|}
\hline \multirow[b]{2}{*}{ Position } & \multicolumn{14}{|c|}{ calculated $^{a}$} & \multirow{2}{*}{$\begin{array}{c}\text { experimental }^{b} \\
1\end{array}$} \\
\hline & 1'F-1 & $1^{\prime} \mathrm{F}-2$ & I'F-3 $^{-1}$ & $1^{\prime} \mathrm{F}-4$ & 1'F-5 & 1'F-6 & 1'F-7 & 1'F-8 & 1'F-9 & 1'F-10 & 1'F-11 & $1^{\prime} \mathrm{F}-12$ & $\begin{array}{c}\text { averaged- } \\
\mathbf{1}^{\prime} \mathbf{F}^{c} \\
\end{array}$ & $\begin{array}{l}\text { averaged-1'F } \\
\text { (corrected) }^{d}\end{array}$ & \\
\hline 1 & 92.8 & 92.8 & 92.6 & 92.7 & 92.7 & 92.7 & 97.0 & 92.8 & 92.8 & 92.8 & 92.7 & 92.8 & 92.8 & 92.2 & 95.6 \\
\hline 2 & 69.9 & 69.9 & 72.2 & 69.9 & 72.1 & 69.9 & 74.7 & 70.0 & 70.0 & 70.0 & 69.9 & 70.0 & 70.6 & 70.3 & 74.1 \\
\hline 3 & 73.6 & 73.6 & 71.3 & 73.6 & 71.3 & 73.6 & 73.3 & 73.6 & 73.6 & 73.6 & 73.6 & 73.6 & 73.0 & 72.7 & 75.9 \\
\hline 4 & 74.9 & 74.9 & 73.9 & 75.0 & 74.0 & 75.0 & 73.5 & 74.8 & 74.9 & 74.8 & 75.0 & 74.9 & 74.6 & 74.3 & 75.4 \\
\hline 5 & 70.0 & 70.0 & 70.2 & 69.9 & 70.2 & 69.9 & 71.9 & 70.0 & 70.0 & 70.0 & 69.9 & 70.0 & 70.1 & 69.8 & 71.2 \\
\hline 6 & 65.4 & 65.3 & 65.6 & 65.3 & 65.6 & 65.2 & 65.7 & 65.3 & 65.4 & 65.2 & 65.3 & 65.4 & 65.4 & 65.2 & 66.5 \\
\hline $1^{\prime}$ & 120.8 & 120.7 & 120.3 & 120.7 & 120.4 & 120.7 & 120.2 & 120.9 & 120.9 & 120.8 & 120.6 & 120.8 & 120.6 & 119.5 & 120.4 \\
\hline $2^{\prime}, 6^{\prime}$ & 109.2 & 109.3 & 109.5 & 109.2 & 109.3 & 109.4 & 109.6 & 109.1 & 109.1 & 109.3 & 109.4 & 109.3 & 109.3 & 108.4 & 110.5 \\
\hline $3^{\prime}, 5^{\prime}$ & 144.3 & 144.5 & 144.5 & 144.3 & 144.5 & 144.5 & 145.2 & 144.3 & 144.4 & 144.5 & 144.5 & 144.5 & 144.5 & 142.9 & 146.5 \\
\hline $4^{\prime}$ & 137.8 & 137.9 & 138.2 & 137.8 & 138.1 & 137.9 & 140.0 & 137.8 & 137.8 & 137.9 & 137.9 & 137.9 & 138.0 & 136.6 & 140.4 \\
\hline $7^{\prime}$ & 168.7 & 168.5 & 168.4 & 168.6 & 168.6 & 168.5 & 172.9 & 168.7 & 168.7 & 168.6 & 168.3 & 168.6 & 168.6 & 166.7 & 166.7 \\
\hline 1" & 123.4 & 123.4 & 123.2 & 121.9 & 122.5 & 121.9 & 123.5 & 123.4 & 122.8 & 123.4 & 122.8 & 122.8 & 123.2 & 122.1 & 115.4 \\
\hline 2" & 121.5 & 121.5 & 121.8 & 121.4 & 121.8 & 121.4 & 121.0 & 121.4 & 121.4 & 121.4 & 121.9 & 121.4 & 121.6 & 120.4 & 123.9 \\
\hline 3" & 108.0 & 108.0 & 108.2 & 108.8 & 108.2 & 108.8 & 107.8 & 108.0 & 108.0 & 108.0 & 109.6 & 108.0 & 108.1 & 107.2 & 108.4 \\
\hline 4" & 147.2 & 147.2 & 147.2 & 147.5 & 147.3 & 147.5 & 147.0 & 147.1 & 147.3 & 147.1 & 147.7 & 147.3 & 147.2 & 145.6 & 146.1 \\
\hline $5^{\prime \prime}$ & 132.4 & 132.5 & 132.5 & 132.6 & 132.4 & 132.7 & 132.2 & 132.4 & 132.2 & 132.4 & 132.9 & 132.3 & 132.4 & 131.1 & 136.2 \\
\hline 6" & 145.8 & 145.8 & 145.8 & 145.0 & 146.2 & 145.1 & 145.8 & 145.6 & 146.2 & 145.6 & 145.4 & 146.3 & 145.8 & 144.2 & 142.6 \\
\hline 7" & 173.8 & 173.9 & 173.8 & 173.7 & 173.8 & 173.7 & 173.6 & 173.8 & 173.9 & 173.8 & 173.5 & 173.9 & 173.8 & 171.8 & 169.4 \\
\hline 1"' & 56.3 & 56.3 & 56.2 & 57.2 & 56.3 & 57.2 & 56.5 & 56.2 & 56.5 & 56.2 & 57.2 & 56.5 & 56.4 & 56.3 & 46.4 \\
\hline $2^{\prime \prime \prime}$ & 45.9 & 45.9 & 45.9 & 46.4 & 46.3 & 46.4 & 46.4 & 46.1 & 46.5 & 46.1 & 45.5 & 46.5 & 46.0 & 46.1 & 48.3 \\
\hline 3"' & 36.3 & 36.3 & 36.2 & 38.6 & 37.7 & 38.6 & 36.4 & 36.7 & 37.7 & 36.7 & 39.3 & 37.7 & 36.6 & 36.9 & 37.8 \\
\hline $4^{\prime \prime \prime}$ & 118.1 & 118.1 & 118.1 & 116.5 & 118.1 & 116.6 & 118.3 & 118.0 & 118.1 & 118.0 & 118.6 & 118.1 & 118.0 & 116.9 & 108.2 \\
\hline 5"' & 83.7 & 83.7 & 83.8 & 85.1 & 85.2 & 85.1 & 83.3 & 85.5 & 85.2 & 85.5 & 83.6 & 85.2 & 84.0 & 83.5 & 80.1 \\
\hline 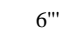 & 178.0 & 178.0 & 178.0 & 176.6 & 175.8 & 176.5 & 177.9 & 174.4 & 175.8 & 174.4 & 176.8 & 175.7 & 177.6 & 175.6 & 172.7 \\
\hline $7^{\prime \prime \prime}$ & 178.2 & 178.2 & 178.1 & 177.9 & 177.6 & 177.9 & 178.2 & 178.2 & 177.7 & 178.2 & 177.8 & 177.7 & 178.1 & 176.0 & 173.7 \\
\hline
\end{tabular}

${ }^{a}$ Calculated using the GIAO method at the mPWIPW91/6-311+G(2d,p) level in MeOH (PCM). ${ }^{b}$ Measured in $\mathrm{CD}{ }_{3} \mathrm{OD}(125 \mathrm{MHz}) .{ }^{c}$ Averaged according to the Boltzmann

distribution theory at $298 \mathrm{~K}$ based on relative Gibbs free energies. ${ }^{d}$ Linearly corrected for the experimental data. 
Table S47. Calculated ${ }^{13} \mathrm{C}$ NMR chemical shifts of $\mathbf{1}^{\prime} \mathbf{G}$.

\begin{tabular}{|c|c|c|c|c|c|c|c|c|c|c|c|}
\hline \multirow[b]{2}{*}{ Position } & \multicolumn{10}{|c|}{ calculated $^{a}$} & \multirow{2}{*}{$\begin{array}{c}\text { experimental }^{b} \\
1\end{array}$} \\
\hline & 1'G-1 & 1'G-2 & 1'G-3 & $1^{\prime}$ G-4 & 1'G-5 & 1'G-6 & $1^{\prime}$ G-7 & 1'G-8 & $\begin{array}{c}\text { averaged- } \\
\mathbf{1}^{\prime} \mathbf{G}^{c}\end{array}$ & $\begin{array}{l}\text { averaged-1'G } \mathbf{G} \\
\text { (corrected) }^{d}\end{array}$ & \\
\hline 1 & 92.1 & 92.1 & $\begin{array}{l}92.9 \\
\end{array}$ & 91.8 & 91.5 & 91.8 & 92.9 & 92.1 & 92.1 & 91.1 & 95.6 \\
\hline 2 & 72.4 & 72.4 & 72.5 & 72.5 & 72.0 & 72.5 & 72.5 & 72.4 & 72.4 & 71.7 & 74.1 \\
\hline 3 & 75.8 & 75.8 & 72.7 & 76.4 & 76.7 & 76.4 & 72.7 & 75.8 & 75.4 & 74.6 & 75.9 \\
\hline 4 & 75.0 & 75.0 & 72.4 & 82.0 & 80.2 & 82.0 & 72.3 & 74.9 & 76.3 & 75.5 & 75.4 \\
\hline 5 & 64.8 & 64.8 & 64.6 & 70.2 & 67.5 & 70.2 & 64.6 & 64.8 & 66.0 & 65.4 & 71.2 \\
\hline 6 & 64.5 & 64.5 & 64.3 & 62.3 & 64.1 & 62.3 & 64.2 & 64.4 & 64.0 & 63.4 & 66.5 \\
\hline $1^{\prime}$ & 120.5 & 120.7 & 120.7 & 119.9 & 119.8 & 119.8 & 120.7 & 120.7 & 120.4 & 118.9 & 120.4 \\
\hline $2^{\prime}, 6^{\prime}$ & 109.5 & 109.2 & 109.1 & 109.7 & 109.9 & 109.5 & 109.1 & 109.2 & 109.4 & 108.1 & 110.5 \\
\hline $3^{\prime}, 5^{\prime}$ & 145.0 & 144.9 & 144.7 & 145.6 & 145.5 & 145.2 & 144.7 & 145.0 & 145.0 & 143.1 & 146.5 \\
\hline $4^{\prime}$ & 138.6 & 138.6 & 138.1 & 139.5 & 139.9 & 139.4 & 138.2 & 138.6 & 138.8 & 136.9 & 140.4 \\
\hline $7^{\prime}$ & 168.9 & 169.0 & 169.6 & 168.5 & 168.3 & 168.3 & 169.6 & 169.0 & 168.9 & 166.5 & 166.7 \\
\hline 1" & 122.6 & 122.6 & 121.2 & 123.5 & 124.4 & 123.5 & 121.2 & 122.7 & 122.7 & 121.1 & 115.4 \\
\hline 2" & 117.8 & 117.8 & 120.5 & 117.9 & 117.3 & 117.9 & 120.4 & 117.8 & 118.3 & 116.8 & 123.9 \\
\hline $3^{\prime \prime}$ & 114.6 & 114.6 & 112.7 & 113.4 & 114.1 & 113.4 & 112.7 & 114.6 & 114.0 & 112.5 & 108.4 \\
\hline 4" & 146.6 & 146.6 & 146.3 & 147.0 & 148.0 & 147.0 & 146.1 & 146.4 & 146.7 & 144.7 & 146.1 \\
\hline $5^{\prime \prime}$ & 134.6 & 134.6 & 132.3 & 135.1 & 135.6 & 135.2 & 131.8 & 134.4 & 134.4 & 132.6 & 136.2 \\
\hline 6" & 146.9 & 146.9 & 146.0 & 145.9 & 146.6 & 145.9 & 145.5 & 146.7 & 146.5 & 144.5 & 142.6 \\
\hline 7" & 173.6 & 173.5 & 171.2 & 175.0 & 173.9 & 174.9 & 171.2 & 173.5 & 173.4 & 170.9 & 169.4 \\
\hline 1"' & 59.3 & 59.3 & 58.0 & 58.5 & 60.1 & 58.5 & 57.9 & 59.1 & 59.0 & 58.5 & 46.4 \\
\hline $2^{\prime \prime \prime}$ & 46.3 & 46.3 & 46.8 & 48.4 & 50.4 & 48.4 & 47.1 & 46.6 & 47.2 & 46.9 & 48.3 \\
\hline 3"' & 36.3 & 36.3 & 35.4 & 37.6 & 34.3 & 37.6 & 36.0 & 36.8 & 36.2 & 36.0 & 37.8 \\
\hline $4^{\prime \prime \prime}$ & 117.7 & 117.7 & 117.7 & 118.0 & 122.2 & 118.0 & 118.1 & 117.9 & 118.2 & 116.7 & 108.2 \\
\hline $5^{\prime \prime \prime}$ & 84.3 & 84.3 & 83.9 & 83.4 & 83.9 & 83.4 & 86.1 & 86.4 & 84.1 & 83.1 & 80.1 \\
\hline 6"' & 177.6 & 177.7 & 177.7 & 177.9 & 173.2 & 177.9 & 174.4 & 174.6 & 177.2 & 174.7 & 172.7 \\
\hline 7"' & 181.8 & 181.8 & 180.4 & 177.7 & 186.0 & 177.7 & 180.6 & 181.9 & 181.2 & 178.6 & 173.7 \\
\hline
\end{tabular}

"Calculated using the GIAO method at the mPWIPW91/6-311+G(2d,p) level in MeOH $(\mathrm{PCM}){ }^{b}$ Measured in $\mathrm{CD}_{3} \mathrm{OD}(125 \mathrm{MH})$.

${ }^{c}$ Averaged according to the Boltzmann distribution theory at $298 \mathrm{~K}$ based on relative Gibbs free energies. ${ }^{d}$ Linearly corrected for the experimental data. 
Table S48. Calculated ${ }^{13} \mathrm{C}$ NMR chemical shifts of $\mathbf{1}^{\prime} \mathbf{H}$.

\begin{tabular}{|c|c|c|c|c|c|c|c|c|c|c|c|c|}
\hline \multirow[b]{2}{*}{ Position } & \multicolumn{11}{|c|}{ calculated $^{a}$} & \multirow{2}{*}{$\begin{array}{c}\text { experimental }^{b} \\
1\end{array}$} \\
\hline & $\mathbf{1}^{\prime} \mathrm{H}-1$ & I'H-2 $^{\prime}$ & $\mathbf{1}^{\prime} \mathrm{H}-3$ & $1^{\prime} \mathrm{H}-4$ & 1'H-5 & $\mathbf{1}^{\prime} \mathrm{H}-6$ & 1'H-7 & 1'H-8 & 1'H-9 & $\begin{array}{c}\text { averaged- } \\
\mathbf{1}^{\prime} \mathbf{H}^{c}\end{array}$ & 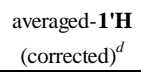 & \\
\hline 1 & 92.2 & 92.1 & 91.6 & 91.6 & 92.2 & 92.2 & 97.1 & 91.6 & 97.0 & 92.2 & 90.7 & 95.6 \\
\hline 2 & 72.3 & 72.3 & 72.2 & 72.3 & 72.3 & 72.3 & 74.5 & 72.4 & 74.6 & 72.4 & 71.1 & 74.1 \\
\hline 3 & 75.9 & 75.9 & 76.6 & 76.5 & 76.0 & 76.0 & 75.0 & 76.4 & 75.0 & 76.0 & 74.7 & 75.9 \\
\hline 4 & 74.8 & 74.8 & 82.7 & 82.6 & 74.8 & 74.8 & 72.3 & 82.3 & 72.3 & 76.3 & 75.0 & 75.4 \\
\hline 5 & 64.9 & 64.9 & 70.4 & 70.3 & 64.9 & 64.9 & 66.1 & 70.2 & 66.1 & 66.0 & 64.9 & 71.2 \\
\hline 6 & 64.4 & 64.4 & 62.3 & 62.2 & 64.3 & 64.3 & 64.3 & 62.1 & 64.3 & 63.9 & 62.8 & 66.5 \\
\hline $1^{\prime}$ & 120.6 & 120.5 & 120.5 & 120.2 & 120.5 & 120.6 & 119.0 & 120.1 & 119.1 & 120.4 & 118.4 & 120.4 \\
\hline $2^{\prime}, 6^{\prime}$ & 109.3 & 109.6 & 109.3 & 109.3 & 109.6 & 109.3 & 110.1 & 109.3 & 109.8 & 109.4 & 107.6 & 110.5 \\
\hline $3^{\prime}, 5^{\prime}$ & 144.9 & 145.1 & 145.4 & 145.0 & 145.1 & 144.9 & 145.0 & 145.1 & 145.1 & 145.0 & 142.6 & 146.5 \\
\hline $4^{\prime}$ & 138.7 & 138.8 & 139.1 & 139.0 & 138.7 & 138.7 & 139.2 & 139.2 & 139.3 & 138.8 & 136.5 & 140.4 \\
\hline $7^{\prime}$ & 169.0 & 168.9 & 169.2 & 168.9 & 168.9 & 169.0 & 172.4 & 168.7 & 172.3 & 169.1 & 166.3 & 166.7 \\
\hline $1 "$ & 121.9 & 122.0 & 122.9 & 122.8 & 122.5 & 122.5 & 120.1 & 123.0 & 120.0 & 122.1 & 120.1 & 115.4 \\
\hline $2^{\prime \prime}$ & 118.1 & 118.1 & 118.7 & 118.6 & 117.9 & 117.9 & 120.4 & 118.4 & 120.6 & 118.3 & 116.3 & 123.9 \\
\hline 3" & 115.5 & 115.5 & 114.7 & 114.7 & 115.0 & 115.1 & 112.9 & 114.3 & 112.9 & 115.2 & 113.3 & 108.4 \\
\hline $4^{\prime \prime}$ & 146.6 & 146.7 & 147.8 & 147.7 & 146.6 & 146.5 & 146.2 & 147.4 & 146.2 & 146.9 & 144.4 & 146.1 \\
\hline 5" & 134.6 & 134.5 & 136.1 & 136.0 & 134.5 & 134.5 & 132.1 & 135.9 & 132.0 & 134.8 & 132.5 & 136.2 \\
\hline 6" & 146.8 & 146.8 & 146.7 & 146.6 & 147.4 & 147.4 & 145.7 & 146.9 & 145.6 & 146.8 & 144.3 & 142.6 \\
\hline 7" & 173.7 & 173.7 & 174.7 & 174.7 & 173.8 & 173.7 & 171.1 & 174.9 & 171.1 & 173.8 & 170.9 & 169.4 \\
\hline 1"' & 61.6 & 61.5 & 60.9 & 61.0 & 61.2 & 61.2 & 60.5 & 59.6 & 60.3 & 61.4 & 60.3 & 46.4 \\
\hline $2^{\prime \prime \prime}$ & 47.8 & 47.8 & 49.8 & 49.9 & 47.3 & 47.2 & 47.8 & 49.0 & 47.8 & 48.2 & 47.3 & 48.3 \\
\hline $3^{\prime \prime \prime}$ & 38.9 & 38.9 & 39.5 & 39.5 & 38.2 & 38.2 & 38.6 & 37.4 & 38.5 & 39.0 & 38.2 & 37.8 \\
\hline $4^{\prime \prime \prime}$ & 122.3 & 122.3 & 121.6 & 121.6 & 122.9 & 122.9 & 122.2 & 121.4 & 122.2 & 122.2 & 120.2 & 108.2 \\
\hline $5 " '$ & 79.6 & 79.6 & 79.5 & 79.5 & 79.0 & 79.0 & 79.5 & 79.3 & 79.4 & 79.5 & 78.2 & 80.1 \\
\hline 6"' & 181.2 & 181.1 & 181.3 & 181.4 & 181.5 & 181.5 & 181.5 & 179.3 & 181.4 & 181.2 & 178.2 & 172.7 \\
\hline 7"' & 181.1 & 181.1 & 177.5 & 177.4 & 181.7 & 181.7 & 180.0 & 177.9 & 180.0 & 180.4 & 177.4 & 173.7 \\
\hline
\end{tabular}

${ }^{a}$ Calculated using the GIAO method at the mPWIPW91/6-311+G(2d,p) level in MeOH $(P C M) .{ }^{b}$ Measured in $\mathrm{CD}_{3} \mathrm{OD}(125 \mathrm{MHz}) .{ }^{c}$ Averaged according to the Boltzmann distribution theory at $298 \mathrm{~K}$ based on relative Gibbs free energies. ${ }^{d}$ Linearly corrected for the experimental data. 
Figure S4. Correlation plots of experimental ${ }^{1} \mathrm{H}$ NMR chemical shifts versus corresponding calculated ${ }^{1} \mathrm{H}$ NMR chemical shifts of $\mathbf{1}$.
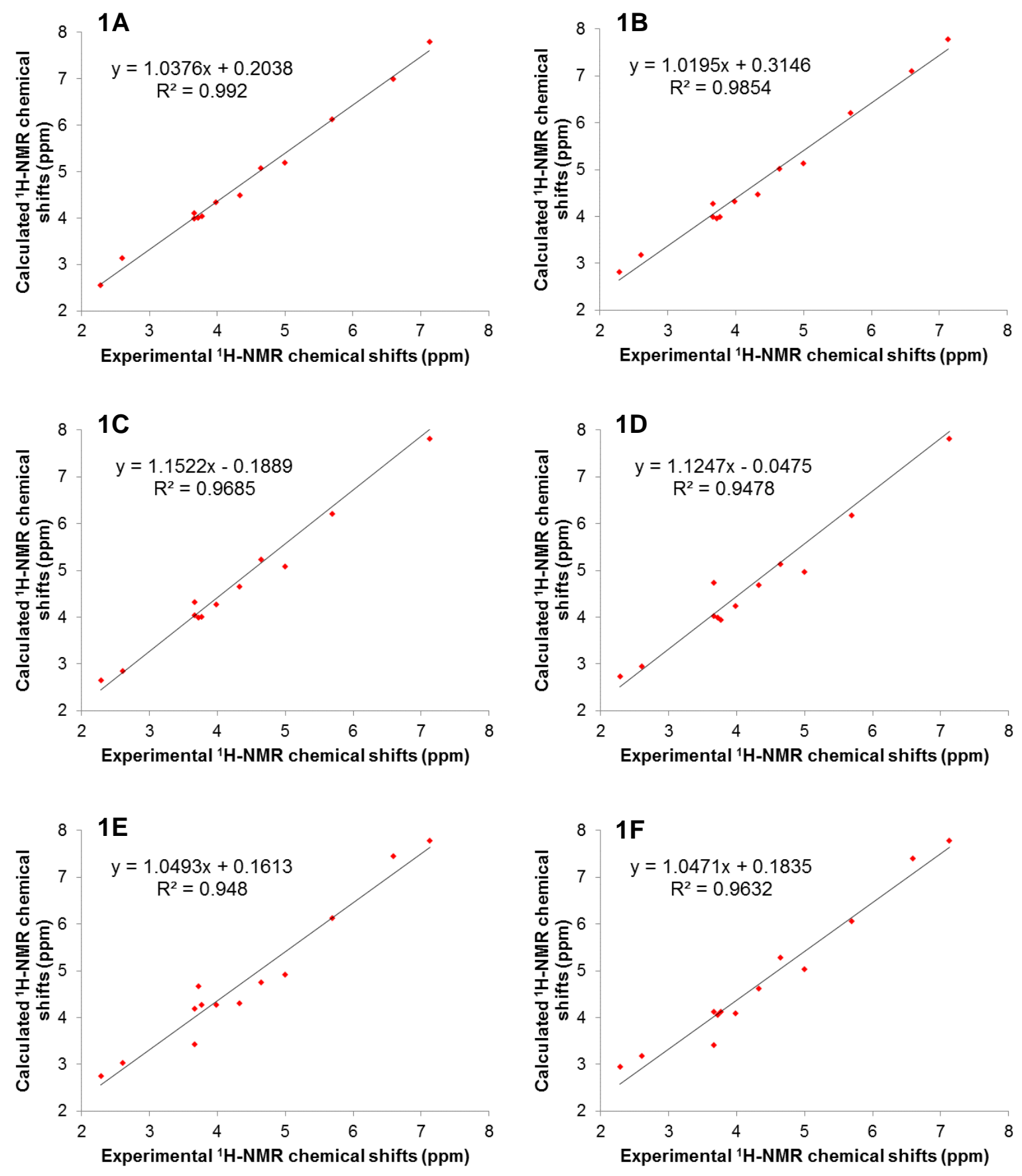

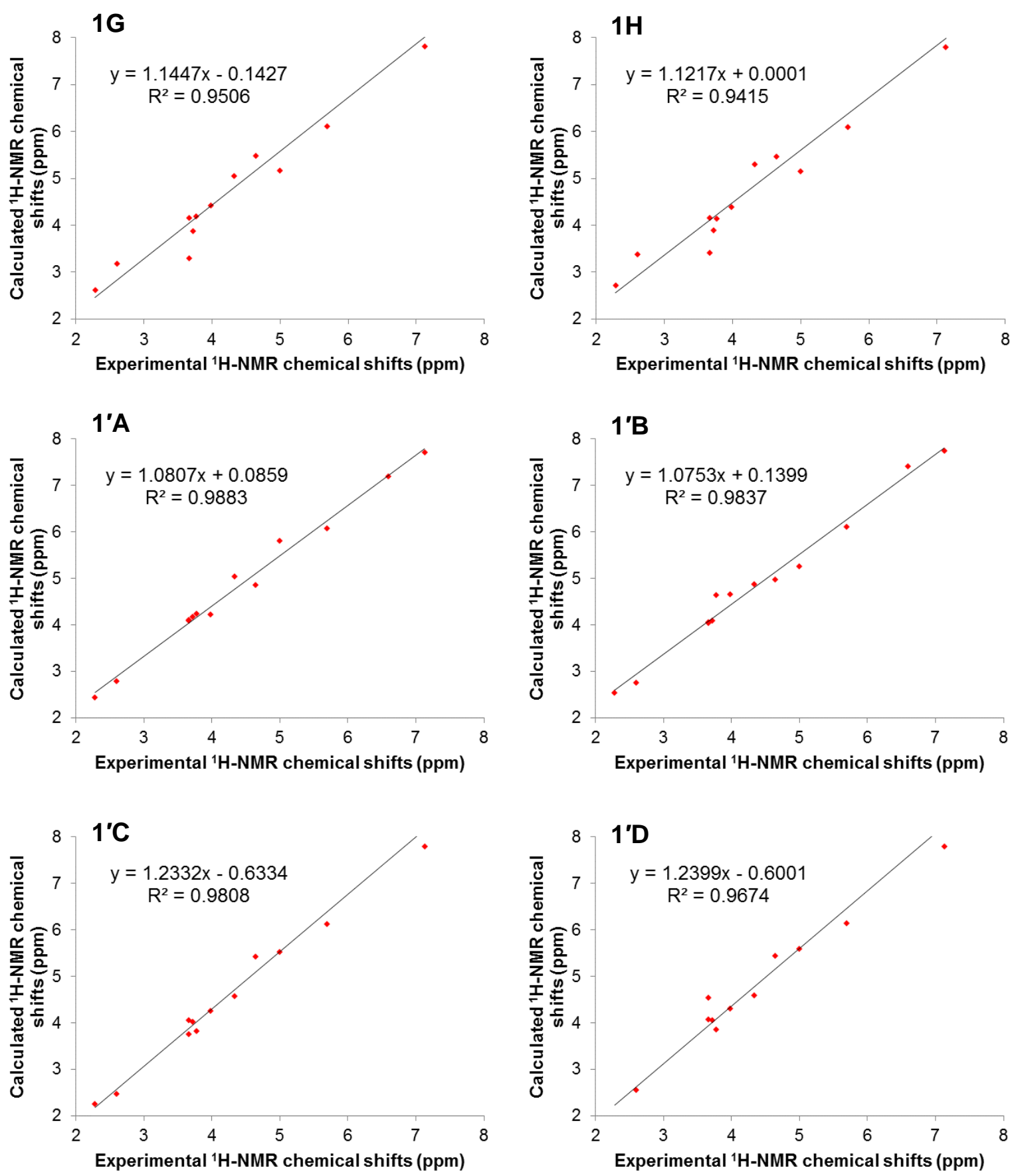

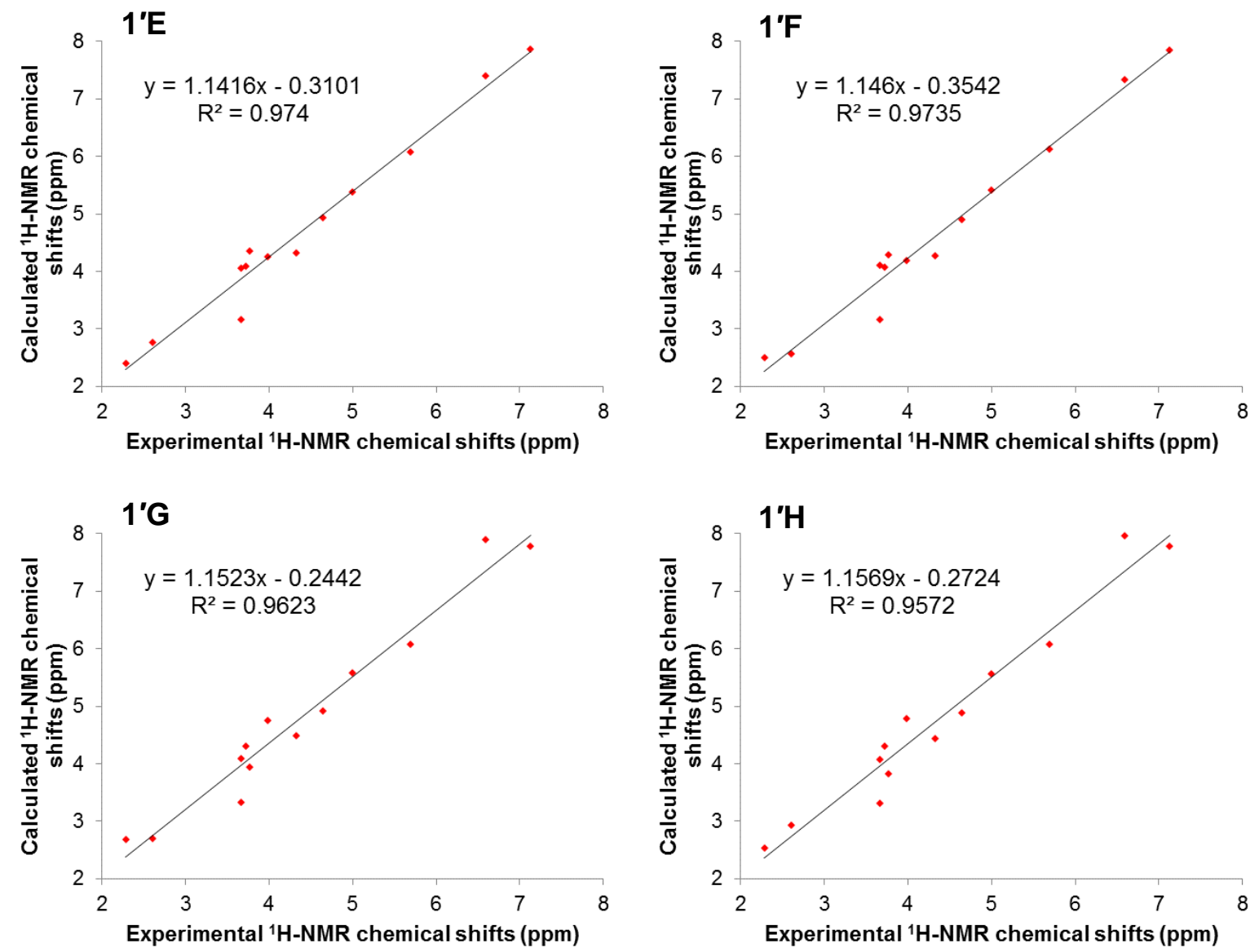
Figure S5. Correlation plots of experimental ${ }^{13} \mathrm{C}$ NMR chemical shifts versus corresponding calculated ${ }^{13} \mathrm{C}$ NMR chemical shifts of $\mathbf{1}$.
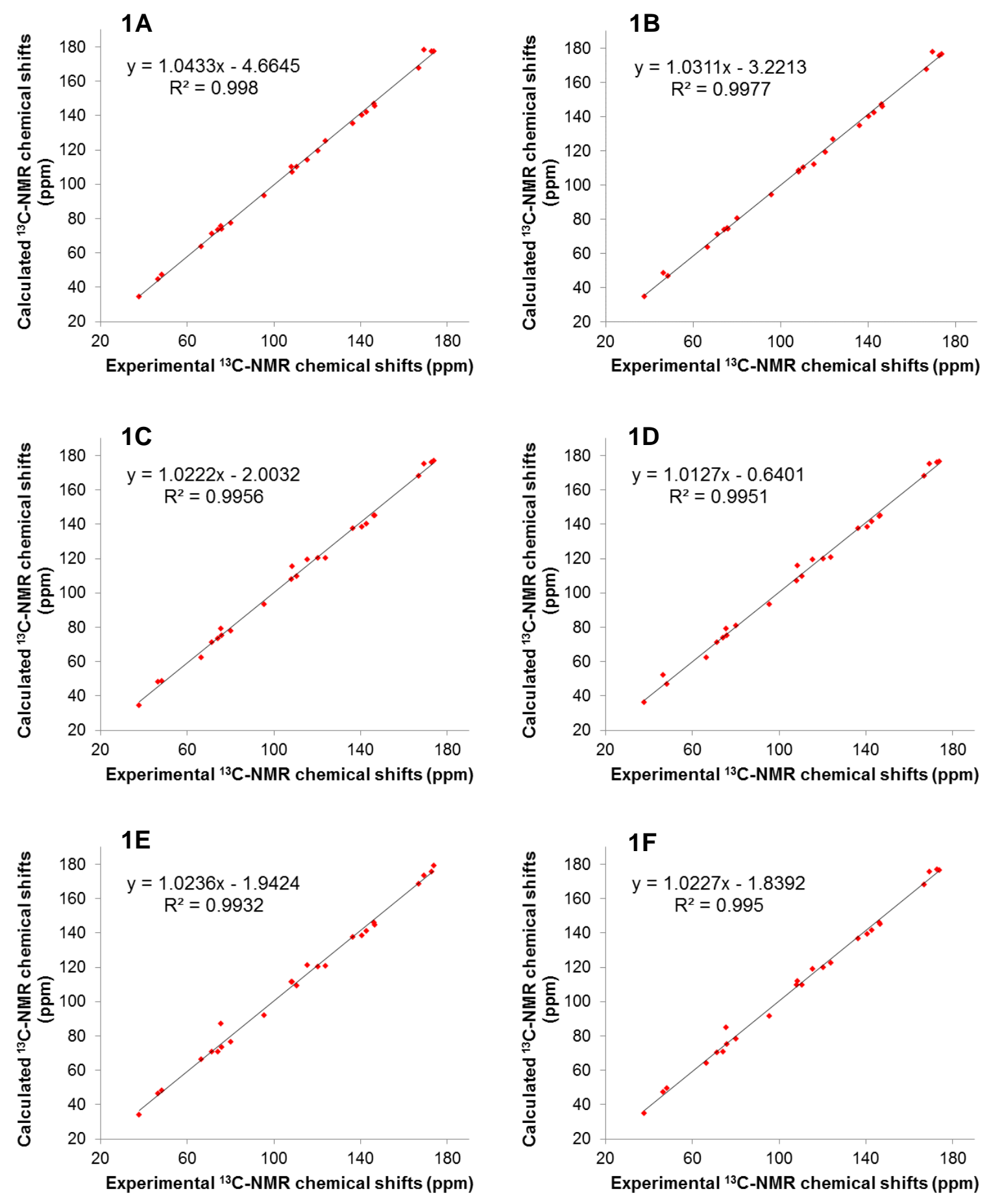

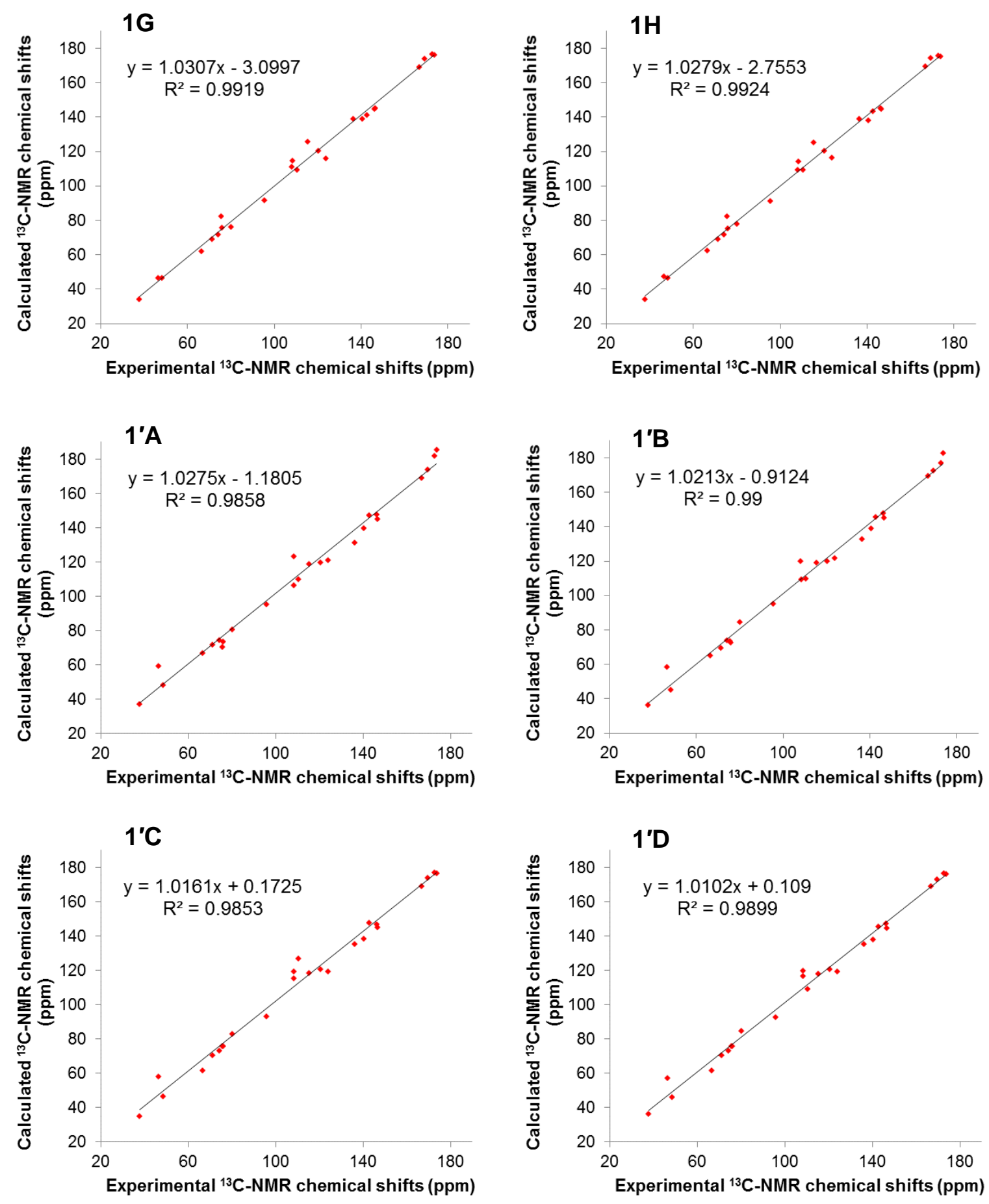

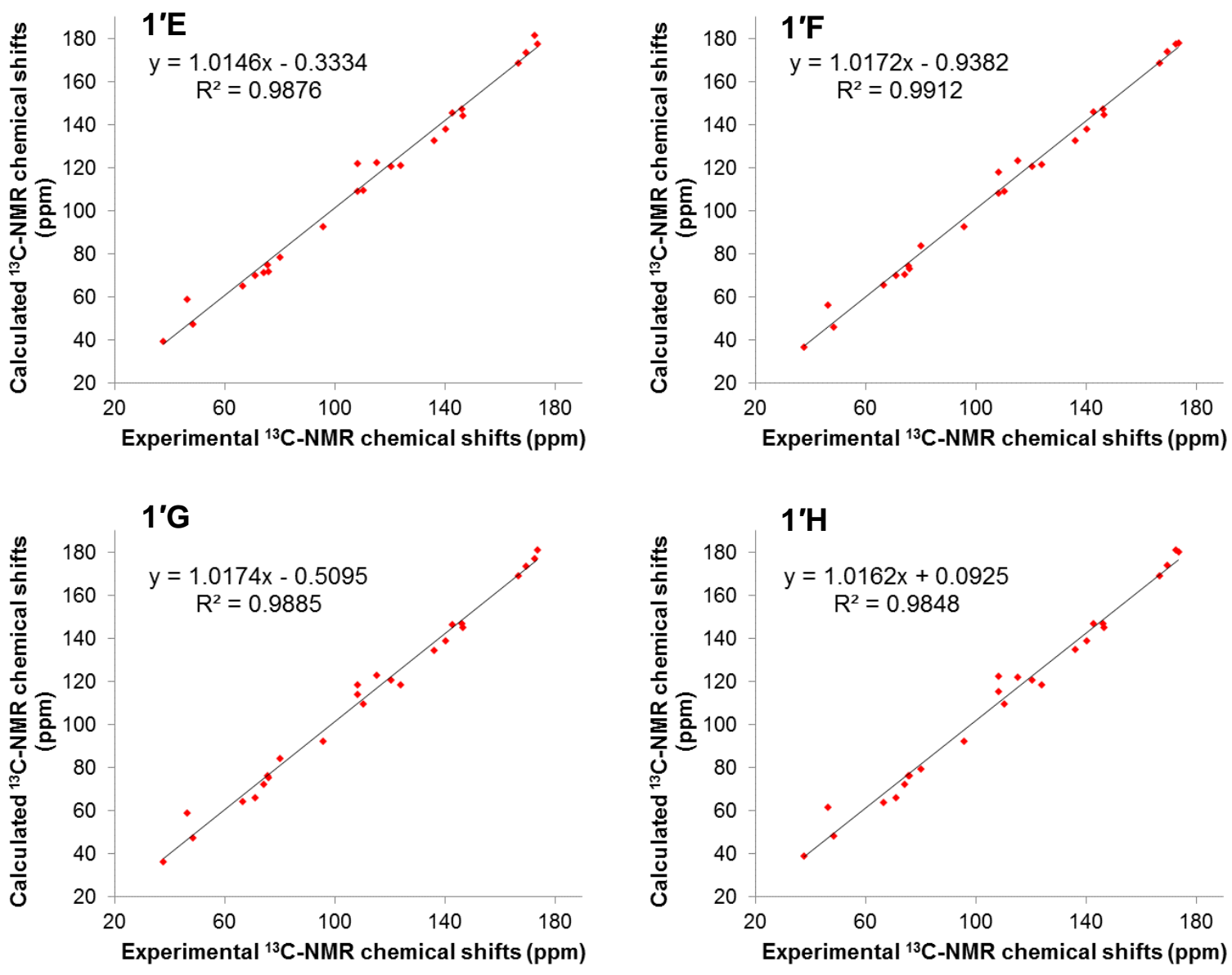
Figure S6. Differences between experimental and calculated ${ }^{13} \mathrm{C}$ NMR chemical shifts of $\mathbf{1}$. Experimental data were measured in $\mathrm{CD}_{3} \mathrm{OD}$. Calculation of NMR chemical shifts were performed at the mPW1PW91/6-311+G(2d,p) level in $\mathrm{MeOH}(\mathrm{PCM})$, and linearly corrected for the experimental data.
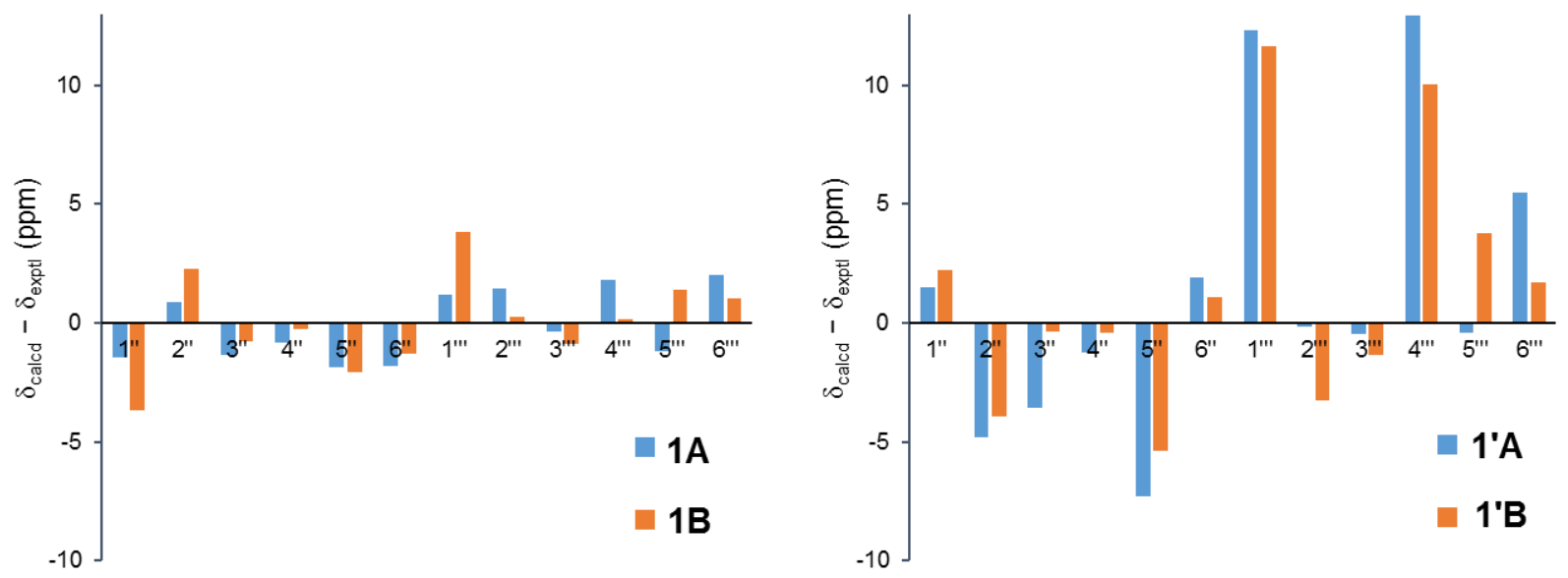
Table S49. Statistical parameters of calculated ${ }^{1} \mathrm{H}$ NMR chemical shifts of $\mathbf{1}(\mathrm{ppm})$.

\begin{tabular}{|c|c|c|c|}
\hline & $R^{2}$ & CMaxErr & CMAE \\
\hline $\mathbf{1 A}$ & 0.9920 & 0.23 & 0.09 \\
\hline $1 B$ & 0.9854 & 0.28 & 0.15 \\
\hline $1 \mathrm{C}$ & 0.9685 & 0.63 & 0.18 \\
\hline $1 D$ & 0.9478 & 0.70 & 0.24 \\
\hline $1 E$ & 0.9480 & 0.58 & 0.27 \\
\hline $1 F$ & 0.9632 & 0.58 & 0.21 \\
\hline $1 G$ & 0.9506 & 0.65 & 0.25 \\
\hline $1 \mathrm{H}$ & 0.9415 & 0.62 & 0.28 \\
\hline 1'A & 0.9883 & 0.29 & 0.12 \\
\hline 1'B & 0.9837 & 0.42 & 0.16 \\
\hline $1^{\prime} \mathrm{C}$ & 0.9808 & 0.43 & 0.15 \\
\hline 1'D & 0.9674 & 0.49 & 0.20 \\
\hline $\mathbf{1}^{\prime} \mathbf{E}$ & 0.9740 & 0.61 & 0.16 \\
\hline $\mathbf{1}^{\prime} \mathbf{F}$ & 0.9735 & 0.59 & 0.16 \\
\hline $1^{\prime} G$ & 0.9623 & 0.55 & 0.23 \\
\hline 1'H & 0.9572 & 0.56 & 0.25 \\
\hline
\end{tabular}

Table S50. Statistical parameters of calculated ${ }^{13} \mathrm{C}$ NMR chemical shifts of $\mathbf{1}(\mathrm{ppm})$.

\begin{tabular}{cccc}
\hline & $R^{2}$ & CMaxErr & CMAE \\
\hline $\mathbf{1 A}$ & $\mathbf{0 . 9 9 8 0}$ & $\mathbf{6 . 0}$ & 1.5 \\
$\mathbf{1 B}$ & 0.9977 & 6.3 & $\mathbf{1 . 4}$ \\
$\mathbf{1 C}$ & 0.9956 & 6.9 & 2.2 \\
$\mathbf{1 D}$ & 0.9951 & 6.6 & 2.3 \\
$\mathbf{1 E}$ & 0.9932 & 11.7 & 2.5 \\
$\mathbf{1 F}$ & 0.9950 & 9.6 & 2.2 \\
$\mathbf{1 G}$ & 0.9919 & 9.5 & 2.8 \\
$\mathbf{1 H}$ & 0.9924 & 9.3 & 2.6 \\
$\mathbf{1} \mathbf{A}$ & 0.9858 & 12.9 & 3.5 \\
$\mathbf{1} \mathbf{B}$ & 0.9900 & 11.6 & 3.0 \\
$\mathbf{1} \mathbf{C}$ & 0.9853 & 14.2 & 3.7 \\
$\mathbf{1} ' \mathbf{D}$ & 0.9899 & 10.4 & 3.1 \\
$\mathbf{1} \mathbf{E}$ & 0.9876 & 12.4 & 3.4 \\
$\mathbf{1} \mathbf{F}$ & 0.9912 & 9.9 & 3.0 \\
$\mathbf{1} ' \mathbf{G}$ & 0.9885 & 12.1 & 3.5 \\
$\mathbf{1}$ 'H & 0.9848 & 13.9 & 3.9 \\
\hline$R^{2}:$ coefficient of determination; CMaxErr: corrected \\
maximum absolute error with respect to the linear fit; CMAE: \\
corrected mean absolute error with respect to the linear fit
\end{tabular}


Table S51. Calculated ${ }^{1} \mathrm{H}$ NMR coupling constants between H-1"' and H-2"' in 1A (ppm).

\begin{tabular}{|c|c|c|c|c|c|c|c|c|c|c|c|c|c|c|}
\hline & & & & & & & calcul & & & & & & & experimental $^{b}$ \\
\hline & 1A-1 & $1 \mathrm{~A}-2$ & 1A-3 & $1 \mathrm{~A}-4$ & $1 \mathrm{~A}-5$ & 1A-6 & 1A-7 & 1A-8 & 1A-9 & $1 \mathrm{~A}-10$ & 1A-11 & $\begin{array}{c}\text { averaged- } \\
\mathbf{1 A}^{c}\end{array}$ & $\begin{array}{l}\text { averaged-1A } \\
{\text { (corrected) }{ }^{d}}^{2}\end{array}$ & 1 \\
\hline$J_{1^{\prime \prime}-2^{\prime \prime \prime}}$ & 6.5 & 6.5 & 6.5 & 5.3 & 6.3 & 5.4 & 5.4 & 6.3 & 0.5 & 6.7 & 6.5 & 6.3 & 5.9 & 5.9 \\
\hline
\end{tabular}

Table S52. Calculated ${ }^{1} \mathrm{H}$ NMR coupling constants between $\mathrm{H}-1^{\prime \prime \prime}$ and $\mathrm{H}-2^{\prime \prime \prime}$ in $\mathbf{1 B}$ (ppm).

\begin{tabular}{|c|c|c|c|c|c|c|c|c|c|c|c|c|c|c|c|c|c|c|}
\hline & & & & & & & & calcula & & & & & & & & & & experimental $^{b}$ \\
\hline 1B-2 & 1B-3 & 1B-4 & 1B-5 & 1B-6 & 1B-7 & 1B-8 & 1B-9 & 1B-10 & 1B-11 & 1B-12 & 1B-13 & 1B-14 & 1B-15 & 1B-16 & 1B-17 & $\begin{array}{c}\text { averaged- } \\
\mathbf{1 B}^{c}\end{array}$ & 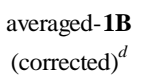 & 1 \\
\hline 6.9 & 6.6 & 7.0 & 6.6 & 7.0 & 5.5 & 6.7 & 5.5 & 5.9 & 6.8 & 5.4 & 5.8 & 6.8 & 5.7 & 7.1 & 7.1 & 6.7 & 6.3 & 5.9 \\
\hline
\end{tabular}

${ }^{a}$ Calculated at the B3LYP/6-31G(d,p)u+1s (using only the Fermi contact term) level in MeOH (PCM). ${ }^{b}$ Measured in $\mathrm{CD}_{3} \mathrm{OD}(500 \mathrm{MHz}) .{ }^{c}$ Averaged according to the Boltzmann distribution theory at $298 \mathrm{~K}$ based on relative Gibbs free energies. ${ }^{d}$ Scaled by using the slope parameter 0.94 .

Table S53. Calculated ${ }^{1} \mathrm{H}$ NMR coupling constants between H-1"' and H-2"' in 1C (ppm).

\begin{tabular}{|c|c|c|c|c|c|c|c|c|c|c|c|}
\hline & \multicolumn{10}{|c|}{ calculated $^{a}$} & \multirow{2}{*}{$\begin{array}{c}\text { experimental } \\
1\end{array}$} \\
\hline & $1 \mathrm{C}-1$ & $1 \mathrm{C}-2$ & $1 \mathrm{C}-3$ & $1 \mathrm{C}-4$ & $1 C-5$ & $1 \mathrm{C}-6$ & $1 \mathrm{C}-7$ & $1 \mathrm{C}-8$ & $\begin{array}{c}\text { averaged- } \\
\mathbf{1 C}^{c}\end{array}$ & $\begin{array}{l}\text { averaged-1C } \\
{\text { (corrected) }{ }^{d}}^{d}\end{array}$ & \\
\hline$J_{1^{\prime \prime \prime}-2^{\prime \prime \prime}}$ & 5.2 & 5.2 & 5.0 & 5.0 & 5.2 & 5.4 & 5.1 & 5.4 & 5.2 & 4.9 & 5.9 \\
\hline
\end{tabular}


Table S54. Calculated ${ }^{1} \mathrm{H}$ NMR coupling constants between $\mathrm{H}-1^{\prime \prime \prime}$ and $\mathrm{H}-2^{\prime \prime \prime}$ in 1D (ppm).

\begin{tabular}{|c|c|c|c|c|c|c|c|c|c|c|c|c|}
\hline & \multicolumn{11}{|c|}{ calculated $^{a}$} & \multirow{2}{*}{$\begin{array}{c}\text { experimental }^{b} \\
1\end{array}$} \\
\hline & 1D-1 & 1D-2 & 1D-3 & 1D-4 & 1D-5 & 1D-6 & 1D-7 & 1D-8 & 1D-9 & $\begin{array}{c}\text { averaged- } \\
\text { 1D }^{c}\end{array}$ & $\begin{array}{l}\text { averaged-1D } \\
{\text { (corrected) })^{d}}^{d}\end{array}$ & \\
\hline$J_{1^{\prime \prime \prime}-2^{\prime \prime}}$ & 5.3 & 5.3 & 5.7 & 5.7 & 5.5 & 5.6 & 5.5 & 5.6 & 5.6 & 5.4 & 5.1 & 5.9 \\
\hline
\end{tabular}

Calculated at the B3LYP/6-3lG(dp)u+ls (using only the Fermi contact term) level in $\mathrm{MeOH}(\mathrm{PCM}){ }^{b}$ Measured in $\mathrm{CD}_{3} \mathrm{OD}(500 \mathrm{MHz})$.

Averaged according to the Boltzmann distribution theory at $298 \mathrm{~K}$ based on relative Gibbs free energies. ${ }^{d}$ Scaled by using the slope parameter 0.94

Table S55. Calculated ${ }^{1} \mathrm{H}$ NMR coupling constants between $\mathrm{H}-1{ }^{\prime \prime \prime}$ and $\mathrm{H}-2^{\prime \prime \prime}$ in $\mathbf{1 E}$ (ppm).

\begin{tabular}{|c|c|c|c|c|c|c|c|c|c|c|c|c|c|c|c|c|c|}
\hline & \multicolumn{16}{|c|}{ calculated $^{a}$} & \multirow{2}{*}{$\frac{\text { experimental }^{b}}{1}$} \\
\hline & 1E-1 & $1 \mathrm{E}-2$ & $1 \mathrm{E}-3$ & $1 \mathrm{E}-4$ & 1E-5 & $1 \mathrm{E}-6$ & 1E-7 & 1E-8 & 1E-9 & 1E-10 & 1E-11 & 1E-12 & 1E-13 & 1E-14 & $\begin{array}{c}\text { averaged- } \\
\mathbf{1 E}^{c} \\
\end{array}$ & $\begin{array}{r}\text { averaged-1E } \\
{\text { (corrected) })^{d}}^{(2)}\end{array}$ & \\
\hline$J_{1^{\prime \prime}-2 \text { '"' }}$ & 1.0 & 1.2 & 1.2 & 1.7 & 1.0 & 1.7 & 0.9 & 0.9 & 1.6 & 1.6 & 1.1 & 1.1 & 1.7 & 1.7 & 1.3 & 1.2 & 5.9 \\
\hline
\end{tabular}

${ }^{a}$ Calculated at the B3LYP/6-31G(d,p)u+1s (using only the Fermi contact term) level in MeOH (PCM). ${ }^{b}$ Measured in $\mathrm{CD}_{3} \mathrm{OD}(500 \mathrm{MHz}) .{ }^{c}$ Averaged according to the Boltzmann distribution theory at 298 K based on relative Gibbs free energies. ${ }^{d}$ Scaled by using the slope parameter 0.94

Table S56. Calculated ${ }^{1} \mathrm{H}$ NMR coupling constants between H-1"' and H-2"' in 1F (ppm).

\begin{tabular}{|c|c|c|c|c|c|c|c|c|c|c|c|c|c|c|c|c|c|c|c|}
\hline & \multicolumn{18}{|c|}{ calculated $^{a}$} & \multirow{2}{*}{$\begin{array}{c}\text { experimental }^{b} \\
1 \\
\end{array}$} \\
\hline & 1F-1 & $1 F-2$ & $1 F-3$ & $1 F-4$ & $1 F-5$ & 1F-6 & 1F-7 & 1F-8 & 1F-9 & 1F-10 & 1F-11 & 1F-12 & 1F-13 & $1 F-14$ & 1F-15 & $1 F-16$ & $\begin{array}{c}\text { averaged- } \\
\mathbf{1 F}^{c} \\
\end{array}$ & $\begin{array}{l}\text { averaged-1F } \\
{\text { (corrected) }{ }^{d}}^{-}\end{array}$ & \\
\hline$J_{1^{\prime \prime \prime}-2^{\prime \prime}}$ & 1.1 & 1.1 & 1.0 & 1.0 & 1.2 & 1.2 & 1.1 & 1.1 & 1.2 & 1.2 & 1.0 & 1.0 & 1.1 & 1.6 & 1.1 & 1.1 & 1.1 & 1.1 & 5.9 \\
\hline
\end{tabular}


Table S57. Calculated ${ }^{1} \mathrm{H}$ NMR coupling constants between $\mathrm{H}-1^{\prime \prime \prime}$ and $\mathrm{H}-2^{\prime \prime \prime}$ in $\mathbf{1 G}$ (ppm).

\begin{tabular}{|c|c|c|c|c|c|c|c|c|c|c|c|c|}
\hline & \multicolumn{11}{|c|}{ calculated $^{a}$} & \multirow{2}{*}{$\begin{array}{c}\text { experimental }^{b} \\
1\end{array}$} \\
\hline & 1G-1 & $1 G-2$ & $1 G-3$ & $1 G-4$ & 1G-5 & 1G-6 & 1G-7 & 1G-8 & 1G-9 & $\begin{array}{c}\text { averaged- } \\
\mathbf{1 G}^{c} \\
\end{array}$ & $\begin{array}{r}\text { averaged-1G }^{\left(\text {corrected }^{d}{ }^{d}\right.} \\
\end{array}$ & \\
\hline$J_{1^{\prime \prime \prime}-2^{2 !}}$ & 1.7 & 1.7 & 1.8 & 1.6 & 1.8 & 1.6 & 1.8 & 1.7 & 1.7 & 1.7 & 1.6 & 5.9 \\
\hline
\end{tabular}

Culculated at the B3LYP/6-31G(dp)u+ls (using only the Fermi contact term) level in $\mathrm{MeOH}(\mathrm{PCM}) .{ }^{b}$ Measured in $\mathrm{CD}_{3} \mathrm{OD}(500 \mathrm{MH})$.

Averaged according to the Boltzmann distribution theory at $298 \mathrm{~K}$ based on relative Gibbs free energies. ${ }^{d}$ Scaled by using the slope parameter 0.94.

Table S58. Calculated ${ }^{1} \mathrm{H}$ NMR coupling constants between H-1"' and H-2"' in $\mathbf{1 H}$ (ppm).

\begin{tabular}{|c|c|c|c|c|c|c|c|c|c|c|c|c|c|c|c|c|c|c|c|c|c|}
\hline & \multicolumn{20}{|c|}{ calculated $^{a}$} & \multirow{2}{*}{$\begin{array}{c}\text { experimental }^{b} \\
1\end{array}$} \\
\hline & 1H-1 & 1H-2 & $1 \mathrm{H}-3$ & $1 \mathrm{H}-4$ & 1H-5 & $1 \mathrm{H}-6$ & $1 \mathrm{H}-7$ & $1 \mathrm{H}-8$ & 1H-9 & $1 \mathrm{H}-10$ & $1 \mathrm{H}-11$ & 1H-12 & $1 \mathrm{H}-13$ & $1 \mathrm{H}-14$ & 1H-15 & $1 \mathrm{H}-16$ & 1H-17 & $1 \mathrm{H}-18$ & $\begin{array}{c}\text { averaged- } \\
1 \mathbf{H}^{c}\end{array}$ & $\begin{array}{c}\text { averaged-1H }^{(\text {corrected })^{d}}\end{array}$ & \\
\hline$J_{1^{\prime \prime \prime}-2{ }^{\prime \prime \prime}}$ & 2.2 & 2.4 & 2.2 & 1.6 & 2.4 & 2.1 & 2.1 & 2.1 & 2.2 & 1.7 & 1.8 & 1.7 & 1.8 & 2.1 & 2.2 & 1.7 & 1.8 & 1.7 & 2.1 & 2.0 & 5.9 \\
\hline
\end{tabular}

${ }^{a}$ Calculated at the B3LYP/6-31G(d,p)u+1s (using only the Fermi contact term) level in MeOH (PCM). ${ }^{b}$ Measured in $\mathrm{CD}{ }_{3} \mathrm{OD}(500 \mathrm{MHz}) .{ }^{c}$ Averaged according to the Boltzmann distribution theory at $298 \mathrm{~K}$ based on relative Gibbs free energies. ${ }^{d}$ Scaled by using the slope parameter 0.94

Table S59. Calculated ${ }^{1} \mathrm{H}$ NMR coupling constants between $\mathrm{H}-1^{\prime \prime \prime}$ and H-2'"' in $\mathbf{1}^{\prime} \mathbf{A}$ (ppm).

\begin{tabular}{|c|c|c|c|c|c|c|c|c|c|}
\hline & \multicolumn{8}{|c|}{ calculated $^{a}$} & \multirow{2}{*}{$\begin{array}{c}\text { experimental }^{b} \\
1\end{array}$} \\
\hline & 1'A-1 & 1'A-2 & $1^{\prime} \mathrm{A}-3$ & $1^{\prime} \mathrm{A}-4$ & $1^{\prime} \mathrm{A}-5$ & 1'A-6 & $\begin{array}{c}\text { averaged- } \\
\mathbf{1}^{\prime} \mathbf{A}^{c}\end{array}$ & $\begin{array}{c}\text { averaged-1'A } \\
{\text { (corrected) }{ }^{d}}^{d}\end{array}$ & \\
\hline$J_{1^{\prime \prime}-2{ }^{2 \prime}}$ & 11.1 & 11.1 & 11.1 & 11.1 & 11.1 & 11.1 & 11.1 & 10.4 & 5.9 \\
\hline
\end{tabular}


Table S60. Calculated ${ }^{1} \mathrm{H}$ NMR coupling constants between $\mathrm{H}-1^{\prime \prime \prime}$ and $\mathrm{H}-\mathbf{2}^{\prime \prime \prime}$ in $\mathbf{1}^{\prime} \mathbf{B}$ (ppm).

\begin{tabular}{|c|c|c|c|c|c|c|c|c|c|c|c|c|c|c|c|}
\hline & \multicolumn{14}{|c|}{ calculated $^{a}$} & \multirow{2}{*}{$\begin{array}{c}\text { experimental } \\
1\end{array}$} \\
\hline & 1'B-1 & 1'B-2 & 1'B-3 & 1'B-4 & 1'B-5 & 1'B-6 & 1'B-7 & 1'B-8 & 1'B-9 & 1'B-10 & 1'B-11 & $1^{\prime} \mathrm{B}-12$ & $\begin{array}{c}\text { averaged- } \\
\mathbf{1}^{\prime} \mathbf{B}^{c} \\
\end{array}$ & $\begin{array}{c}\text { averaged-1'B } \mathbf{B} \\
{\text { (corrected) }{ }^{d}}^{d}\end{array}$ & \\
\hline$J_{1{ }^{\prime \prime \prime}-2 ! "}$ & 12.0 & 12.0 & 12.0 & 9.1 & 8.8 & 8.7 & 8.8 & 9.2 & 9.2 & 8.7 & 9.2 & 9.0 & 10.9 & 10.3 & 5.9 \\
\hline
\end{tabular}

${ }^{a}$ Calculated at the B3LYP/6-31G(d,p)u+1s (using only the Fermi contact term) level in MeOH (PCM). ${ }^{b}$ Measured in $\mathrm{CD}_{3} \mathrm{OD}(500 \mathrm{MHz}) .{ }^{c}$ Averaged according to the Boltzmann

distribution theory at $298 \mathrm{~K}$ based on relative Gibbs free energies. ${ }^{d}$ Scaled by using the slope parameter 0.94

Table S61. Calculated ${ }^{1} \mathrm{H}$ NMR coupling constants between $\mathrm{H}-1^{\prime \prime \prime}$ and $\mathrm{H}-\mathbf{2}^{\prime \prime \prime}$ in $\mathbf{1}^{\prime} \mathbf{C}(\mathrm{ppm})$.

\begin{tabular}{|c|c|c|c|c|c|c|c|c|c|c|c|c|c|c|c|c|c|c|c|}
\hline & \multicolumn{18}{|c|}{ calculated $^{a}$} & \multirow{2}{*}{$\begin{array}{c}\text { experimental }^{b} \\
1 \\
\end{array}$} \\
\hline & $1^{\prime} \mathrm{C}-1$ & $1^{\prime} \mathrm{C}-2$ & $1^{\prime} \mathrm{C}-3$ & $1^{\prime} \mathrm{C}-4$ & $1^{\prime} \mathrm{C}-5$ & $1^{\prime} \mathrm{C}-6$ & 1'C-7 & $1^{\prime} \mathrm{C}-8$ & $1^{\prime} \mathrm{C}-9$ & $1^{\prime} \mathrm{C}-10$ & $1^{\prime} \mathrm{C}-11$ & $1^{\prime} \mathrm{C}-12$ & $1^{\prime} \mathrm{C}-13$ & $1^{\prime} \mathrm{C}-14$ & $1^{\prime} \mathrm{C}-15$ & $1^{\prime} \mathrm{C}-16$ & $\begin{array}{c}\text { averaged- } \\
\mathbf{1}^{\prime} \mathbf{C}^{c}\end{array}$ & 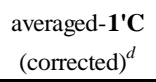 & \\
\hline$J_{1^{\prime \prime \prime}-2^{\prime \prime}}$ & 7.5 & 7.5 & 7.7 & 8.3 & 9.4 & 9.4 & 8.3 & 7.7 & 9.6 & 9.5 & 8.2 & 7.5 & 8.2 & 7.3 & 7.3 & 7.5 & 8.0 & 7.5 & 5.9 \\
\hline
\end{tabular}

${ }^{a}$ Calculated at the B3LYP//6-31 G(d,p)u+1s (using only the Fermi contact term) level in MeOH $(P C M) .{ }^{b}$ Measured in $C D{ }_{3} \mathrm{OD}(500 \mathrm{MHz})$. ${ }^{c}$ Averaged according to the Boltzmann distribution theory at $298 \mathrm{~K}$ based on

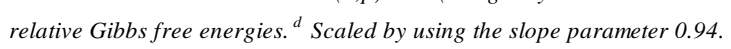

Table S62. Calculated ${ }^{1} \mathrm{H}$ NMR coupling constants between H-1"' and H-2"' in 1'D (ppm).

\begin{tabular}{|c|c|c|c|c|c|c|c|c|c|c|c|c|c|}
\hline & \multicolumn{12}{|c|}{ calculated $^{a}$} & \multirow{2}{*}{$\begin{array}{c}\text { experimental }^{b} \\
1\end{array}$} \\
\hline & 1'D-1 & 1'D-2 & 1'D-3 & 1'D-4 & 1'D-5 & 1'D-6 & 1'D-7 & 1'D-8 & 1'D-9 & 1'D-10 & $\begin{array}{c}\text { averaged- } \\
\mathbf{1}^{\prime} \mathbf{D}^{c}\end{array}$ & $\begin{array}{c}\text { averaged-1'D } \\
\text { (corrected) }^{d}\end{array}$ & \\
\hline$J_{1^{\prime \prime \prime}-2^{\prime \prime \prime}}$ & 8.6 & 8.6 & 8.4 & 9.2 & 9.1 & 9.1 & 8.9 & 8.5 & 7.4 & 7.4 & 8.6 & 8.1 & 5.9 \\
\hline
\end{tabular}

${ }^{a}$ Calculated at the B3LYP/6-31G(d,p)u+1s (using only the Fermi contact term) level in MeOH (PCM). ${ }^{b}$ Measured in $\mathrm{CD}_{3} \mathrm{OD}(50 \mathrm{MHz}) .{ }^{c}$ Averaged

according to the Boltzmann distribution theory at $298 \mathrm{~K}$ based on relative Gibbs free energies. ${ }^{d}$ Scaled by using the slope parameter 0.94. 
Table S63. Calculated ${ }^{1} \mathrm{H}$ NMR coupling constants between $\mathrm{H}-1^{\prime \prime \prime}$ and $\mathrm{H}-2^{\prime \prime \prime}$ in $\mathbf{1}$ 'E (ppm).

\begin{tabular}{|c|c|c|c|c|c|c|c|c|c|c|c|c|}
\hline & \multicolumn{11}{|c|}{ calculated $^{a}$} & \multirow{2}{*}{$\begin{array}{c}\text { experimental }^{b} \\
1\end{array}$} \\
\hline & 1'E-1 & $1^{\prime} \mathrm{E}-2$ & 1'E-3 & 1'E-4 & 1'E-5 & 1'E-6 & 1'E-7 & 1'E-8 & 1'E-9 & $\begin{array}{c}\text { averaged- } \\
\mathbf{1}^{\prime} \mathbf{E}^{c} \\
\end{array}$ & $\begin{array}{c}\text { averaged-1'E } \\
\text { (corrected) }^{d}\end{array}$ & \\
\hline$J_{1^{\prime \prime \prime}-2^{\prime \prime \prime}}$ & 10.5 & 10.5 & 10.5 & 10.5 & 9.4 & 10.5 & 10.5 & 10.5 & 10.5 & 10.4 & 9.8 & 5.9 \\
\hline
\end{tabular}

${ }^{a}$ Calculated at the B3LYP/6-31G(d,p)u+1s (using only the Fermi contact term) level in MeOH (PCM). ${ }^{b}$ Measured in $\mathrm{CD}{ }_{3} \mathrm{OD}(50 \mathrm{MHz}) .{ }^{c}$

Averaged according to the Boltzmann distribution theory at $298 \mathrm{~K}$ based on relative Gibbs free energies. ${ }^{d}$ Scaled by using the slope parameter 0.94.

Table S64. Calculated ${ }^{1} \mathrm{H}$ NMR coupling constants between $\mathrm{H}-1^{\prime \prime \prime}$ and $\mathrm{H}-\mathbf{2}^{\prime \prime \prime}$ in $\mathbf{1}^{\prime} \mathbf{F}$ (ppm).

\begin{tabular}{|c|c|c|c|c|c|c|c|c|c|c|c|c|c|c|c|}
\hline & \multicolumn{14}{|c|}{ calculated $^{a}$} & \multirow{2}{*}{$\begin{array}{c}\text { experimental } \\
1 \\
\end{array}$} \\
\hline & 1'F-1 & 1'F-2 & $1^{\prime} F-3$ & I'F-4 $^{\prime}$ & 1'F-5 & 1'F-6 & 1'F-7 & 1'F-8 & 1'F-9 & 1'F-10 & 1'F-11 & 1'F-12 & $\begin{array}{c}\text { averaged- } \\
\mathbf{1}^{\prime} \mathbf{F}^{c} \\
\end{array}$ & $\begin{array}{l}\text { averaged-1'F } \\
\text { (corrected) }^{d}\end{array}$ & \\
\hline$J_{1^{\prime \prime \prime}-2^{\prime \prime \prime}}$ & 10.5 & 10.5 & 10.6 & 10.3 & 10.6 & 10.3 & 10.5 & 10.6 & 10.5 & 10.6 & 11.0 & 10.5 & 10.5 & 9.9 & 5.9 \\
\hline
\end{tabular}

${ }^{a}$ Calculated at the B3LYP/6-31G(d,p)u+1s (using only the Fermi contact term) level in $\mathrm{MeOH}(\mathrm{PCM}) .{ }^{b}$ Measured in $\mathrm{CD}{ }_{3} \mathrm{OD}(500 \mathrm{MHz}) .{ }^{c}$ Averaged according to the Boltzmann distribution theory at $298 \mathrm{~K}$ based on relative Gibbs free energies. ${ }^{d}$ Scaled by using the slope parameter 0.94.

Table S65. Calculated ${ }^{1} \mathrm{H}$ NMR coupling constants between H-1"' and H-2"' in $\mathbf{1}^{\prime} \mathbf{G}$ (ppm).

\begin{tabular}{|c|c|c|c|c|c|c|c|c|c|c|c|}
\hline & \multicolumn{10}{|c|}{ calculated $^{a}$} & \multirow{2}{*}{$\begin{array}{c}\text { experimental }^{b} \\
1\end{array}$} \\
\hline & $1 ' G-1$ & $1^{\prime} \mathrm{G}-2$ & $1 ' G-3$ & $1 ' G-4$ & $1 ' G-5$ & $1^{\prime} \mathrm{G}-6$ & 1'G-7 & 1'G-8 & $\begin{array}{c}\text { averaged- } \\
\mathbf{1}^{\prime} \mathbf{G}^{c}\end{array}$ & $\begin{array}{c}\text { averaged-1' } \mathbf{G} \\
{\text { (corrected })^{d}}^{d}\end{array}$ & \\
\hline$J_{1^{1 "}-2{ }^{2 !}}$ & 8.4 & 8.4 & 8.7 & 11.0 & 4.4 & 11.0 & 8.6 & 8.3 & 8.5 & 8.0 & 5.9 \\
\hline
\end{tabular}


Table S66. Calculated ${ }^{1} \mathrm{H}$ NMR coupling constants between $\mathrm{H}-1^{\prime \prime \prime}$ and $\mathrm{H}-\mathbf{2}^{\prime \prime \prime}$ in $\mathbf{1}^{\prime} \mathbf{H}$ (ppm).

\begin{tabular}{|c|c|c|c|c|c|c|c|c|c|c|c|c|}
\hline & \multicolumn{11}{|c|}{ calculated $^{a}$} & \multirow{2}{*}{$\begin{array}{c}\text { experimental }^{b} \\
1\end{array}$} \\
\hline & $1^{\prime} \mathrm{H}-1$ & $\mathbf{1}^{\prime} \mathrm{H}-2$ & $\mathbf{1}^{\prime} \mathrm{H}-3$ & $1^{\prime} \mathrm{H}-4$ & 1'H-5 $^{\prime}$ & 1'H-6 & $1^{\prime} \mathrm{H}-7$ & 1'H-8 & 1'H-9 & $\begin{array}{c}\text { averaged- } \\
\mathbf{1}^{\prime} \mathbf{H}^{c}\end{array}$ & $\begin{array}{c}\text { averaged-1'H } \mathbf{H} \\
{\text { (corrected) }{ }^{d}}^{d}\end{array}$ & \\
\hline$J_{1^{\prime \prime \prime}-2^{\prime \prime \prime}}$ & 8.3 & 8.3 & 10.6 & 10.6 & 8.2 & 8.2 & 8.4 & 10.7 & 8.4 & 8.7 & 8.2 & 5.9 \\
\hline
\end{tabular}

${ }^{a}$ Calculated at the B3LYP/6-31G(d,p)u+1s (using only the Fermi contact term) level in MeOH (PCM). ${ }^{b}$ Measured in $\mathrm{CD}_{3} \mathrm{OD}(50 \mathrm{MHz}) .{ }^{c}$

Averaged according to the Boltzmann distribution theory at $298 \mathrm{~K}$ based on relative Gibbs free energies. ${ }^{d}$ Scaled by using the slope parameter 0.94. 
Table S67. Important thermodynamic parameters and conformational analysis of 2 at the B3LYP/6-31G(d,p) level in MeOH (PCM).

\begin{tabular}{clllllr}
\hline conformers & $E$ (a.u.) & $E^{\prime}$ (a.u.) & $H$ (a.u.) & $G$ (a.u.) & $\Delta G(\mathrm{kcal} / \mathrm{mol})$ & $\mathrm{P}_{\mathrm{G}}(\%)$ \\
\hline 2-A & -1065.510970 & -1065.294550 & -1065.276519 & -1065.339455 & 0.00 & 39.2 \\
2-B & -1065.510018 & -1065.293527 & -1065.275357 & -1065.339134 & 0.20 & 27.9 \\
2-C & -1065.507318 & -1065.291386 & -1065.272776 & -1065.337923 & 0.96 & 7.7 \\
2-D & -1065.506493 & -1065.290533 & -1065.271869 & -1065.337424 & 1.27 & 4.6 \\
2-E & -1065.505459 & -1065.289873 & -1065.270871 & -1065.337041 & 1.51 & 3.0 \\
2-F & -1065.506141 & -1065.290154 & -1065.271446 & -1065.336789 & 1.67 & 2.3 \\
2-G & -1065.507195 & -1065.290835 & -1065.272462 & -1065.336667 & 1.75 & 2.0 \\
2-H & -1065.505323 & -1065.289175 & -1065.270481 & -1065.336583 & 1.80 & 1.9 \\
2-I & -1065.504841 & -1065.289247 & -1065.270317 & -1065.336511 & 1.85 & 1.7 \\
2-J & -1065.505842 & -1065.289871 & -1065.271222 & -1065.336398 & 1.92 & 1.5 \\
2-K & -1065.502826 & -1065.287838 & -1065.268357 & -1065.336322 & 1.97 & 1.4 \\
2-L & -1065.505118 & -1065.289519 & -1065.270554 & -1065.336300 & 1.98 & 1.4 \\
2-M & -1065.504387 & -1065.288884 & -1065.269958 & -1065.336274 & 2.00 & 1.3 \\
2-N & -1065.504896 & -1065.289051 & -1065.270383 & -1065.336254 & 2.01 & 1.3 \\
2-O & -1065.505910 & -1065.290264 & -1065.271371 & -1065.336236 & 2.02 & 1.3 \\
2-P & -1065.503130 & -1065.288037 & -1065.268640 & -1065.336223 & 2.03 & 1.3 \\
\hline
\end{tabular}

E: total energy; $E^{\prime}$ : total energy with zero point energy; H: enthalpy; G: Gibbs free energy; $\Delta G$ : relative Gibbs free energy at the B3LYP/6-311+G(2d,p) level in $\mathrm{MeOH}(P C M) . P_{G}$ : conformational distribution calculated from relative Gibbs free energy. 
Table 68. Calculated optical rotations for $\mathbf{2}$

\begin{tabular}{|c|c|c|}
\hline conformers & $\operatorname{Exptl}[\alpha]_{\mathrm{D}}^{a}$ & Calcd $[\alpha]_{\mathrm{D}}^{b, c}$ \\
\hline $2-A$ & & -407.9 \\
\hline 2-B & & +119.6 \\
\hline 2-C & & -34.9 \\
\hline 2-D & & -27.1 \\
\hline 2-E & & +155.8 \\
\hline 2-F & & -102.9 \\
\hline 2-G & & +227.8 \\
\hline 2-H & & -98.4 \\
\hline $2-I$ & & -131.3 \\
\hline 2-J & & -37.4 \\
\hline $2-K$ & & +19.9 \\
\hline 2-L & & +222.9 \\
\hline 2-M & & +92.0 \\
\hline $2-N$ & & -99.3 \\
\hline 2-O & & -286.1 \\
\hline 2-P & & -104.6 \\
\hline 2 (averaged) $^{d}$ & & -129.9 \\
\hline 2 (exptl) & -26.8 & \\
\hline \multicolumn{3}{|c|}{$\begin{array}{l}{ }^{a} \text { measured in } \mathrm{MeOH},{ }^{b} \text { calculated by using frequency } \\
\text { dependent polarizabilities at } 589.3 \mathrm{~nm},{ }^{c} \mathrm{~B} 3 \mathrm{LYP} / 6- \\
311+G(2 d, p) / / \mathrm{B} 3 \mathrm{LYP} / 6-311+G(2 d, p) \text { in } \mathrm{MeOH} \\
(P C M),{ }^{d} \text { Averaged according to the Boltzmann } \\
\text { distribution theory at } 298 \mathrm{~K} \text { based on relative Gibbs } \\
\text { free energies. }\end{array}$} \\
\hline
\end{tabular}


Figure S7. Possible structures of penthorumnin C (3).

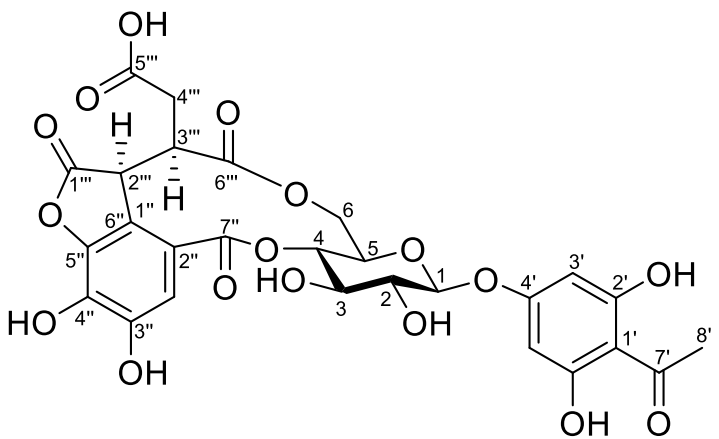

3A

(2"'S, 3"'R)

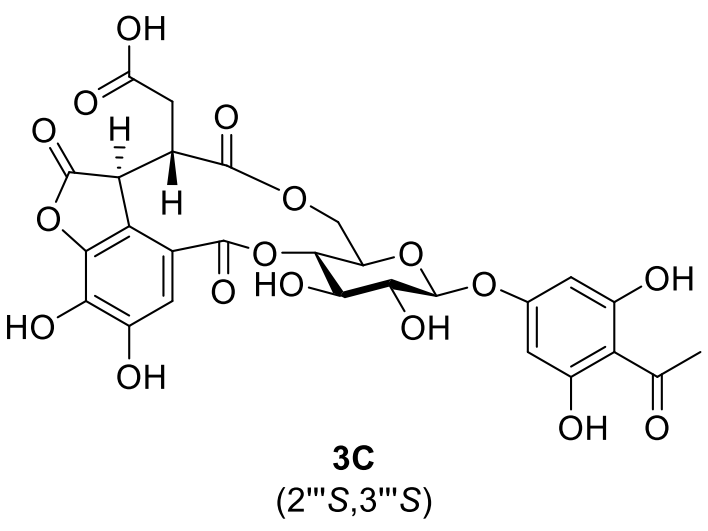

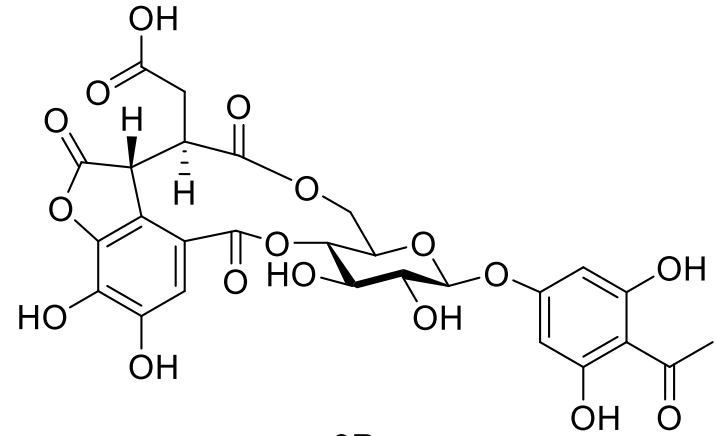

3B

(2"'R,3"'R)

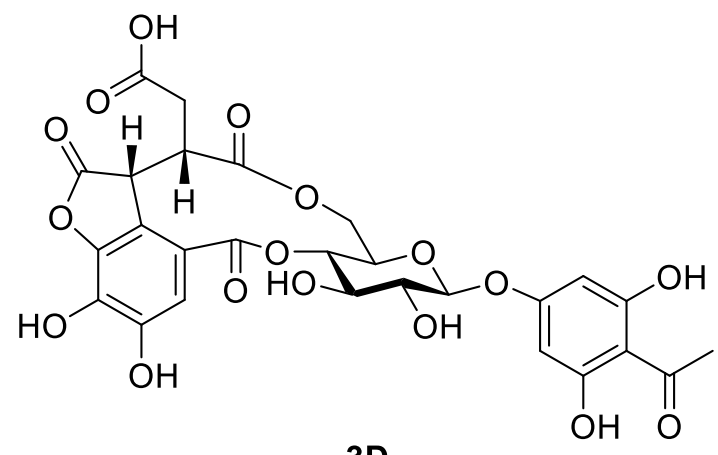

3D

(2'"R,3"'S) 
Figure S8. Lowest-energy conformers of $\mathbf{3}$ and Boltzmann-weighted calculated $J_{2^{\prime \prime \prime}-3^{\prime \prime \prime}}$ values (B3LYP/6-31G(d,p)u+1s level in acetone (PCM)).

$J_{\text {exptl }}\left(J_{\text {calcd }}\right)$ in $\mathrm{Hz}$
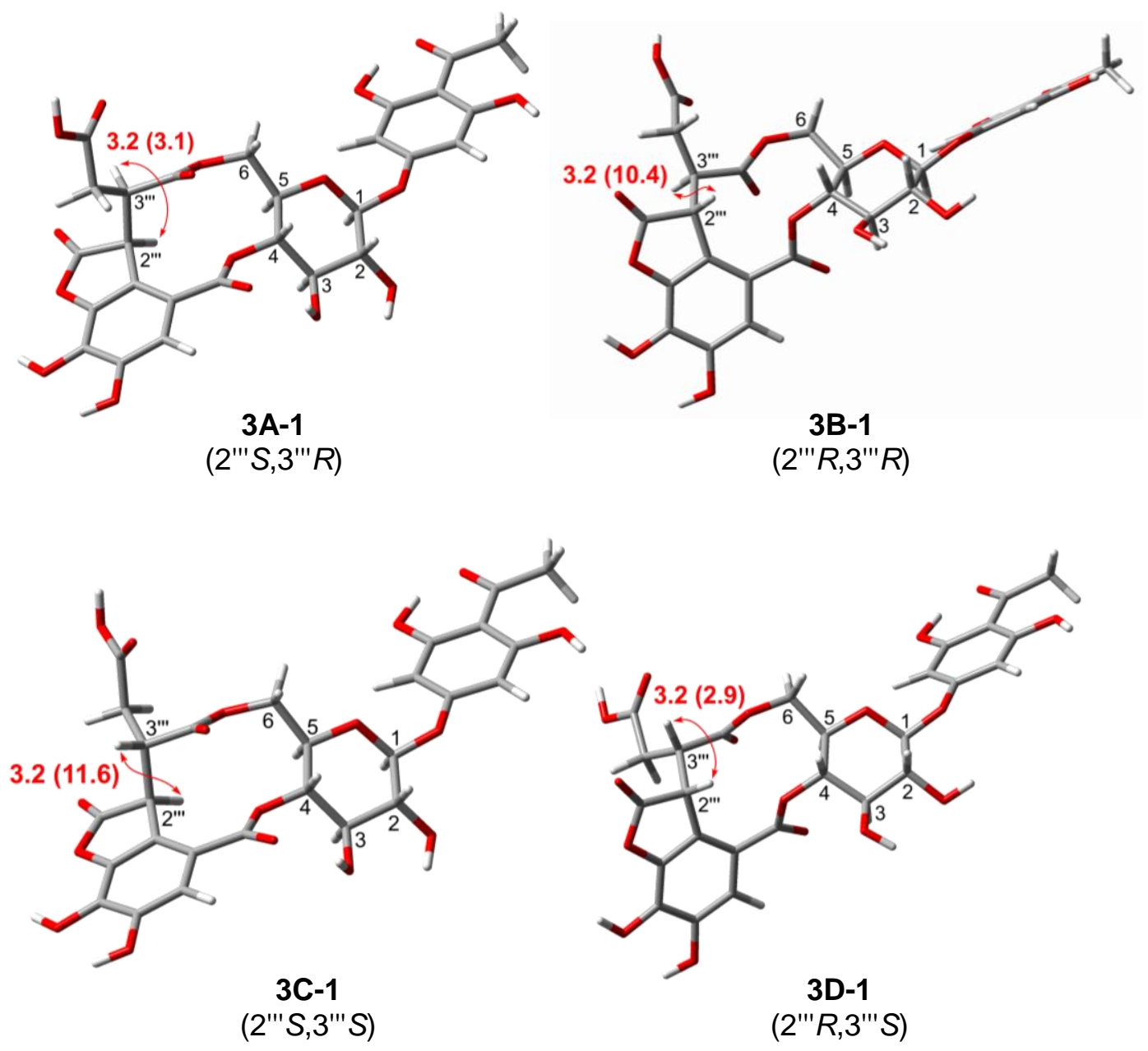
Table S69. Important thermodynamic parameters and conformational analysis of $\mathbf{3 A}$ at the B3LYP/6-31G(d,p) level in acetone (PCM).

\begin{tabular}{ccccccc}
\hline conformers & $E$ (a.u.) & $E^{\prime}$ (a.u.) & $H$ (a.u.) & $G$ (a.u.) & $\Delta G(\mathrm{kcal} / \mathrm{mol})$ & $\mathrm{P}_{\mathrm{G}}(\%)$ \\
\hline $\mathbf{3 A - 1}$ & -2322.235516 & -2321.729357 & -2321.689344 & -2321.802598 & 0.00 & 27.0 \\
$\mathbf{3 A - 2}$ & -2322.234765 & -2321.728671 & -2321.688634 & -2321.801952 & 0.41 & 13.6 \\
$\mathbf{3 A - 3}$ & -2322.233439 & -2321.727991 & -2321.687600 & -2321.801942 & 0.41 & 13.5 \\
$\mathbf{3 A - 4}$ & -2322.232888 & -2321.727602 & -2321.687167 & -2321.801762 & 0.52 & 11.1 \\
$\mathbf{3 A - 5}$ & -2322.233805 & -2321.727999 & -2321.687789 & -2321.801542 & 0.66 & 8.8 \\
$\mathbf{3 A - 6}$ & -2322.233203 & -2321.727606 & -2321.687324 & -2321.801511 & 0.68 & 8.5 \\
$\mathbf{3 A - 7}$ & -2322.233260 & -2321.727284 & -2321.687194 & -2321.801134 & 0.92 & 5.7 \\
$\mathbf{3 A - 8}$ & -2322.232446 & -2321.726545 & -2321.686406 & -2321.800631 & 1.23 & 3.4 \\
$\mathbf{3 A - 9}$ & -2322.231518 & -2321.725804 & -2321.685543 & -2321.799845 & 1.73 & 1.5 \\
$\mathbf{3 A - 1 0}$ & -2322.231161 & -2321.725757 & -2321.685334 & -2321.799804 & 1.75 & 1.4 \\
$\mathbf{3 A - 1 1}$ & -2322.230854 & -2321.725327 & -2321.684999 & -2321.799717 & 1.81 & 1.3 \\
\hline
\end{tabular}

E: total energy; $E$ ': total energy with zero point energy; H: enthalpy; G: Gibbs free energy; $\triangle$ G: relative Gibbs free energy at the B3LYP/6-31G(d,p) level in acetone $(P C M) . P_{G}$ : conformational distribution calculated from relative Gibbs free energy.

Table S70. Important thermodynamic parameters and conformational analysis of $\mathbf{3 B}$ at the B3LYP/6-31G $(\mathrm{d}, \mathrm{p})$ level in acetone (PCM).

\begin{tabular}{ccccccc}
\hline conformers & $E$ (a.u.) & $E^{\prime}$ (a.u.) & $H$ (a.u.) & $G$ (a.u.) & $\Delta G(\mathrm{kcal} / \mathrm{mol})$ & $\mathrm{P}_{\mathrm{G}}(\%)$ \\
\hline 3B-1 & -2322.225663 & -2321.719955 & -2321.679656 & -2321.793649 & 0.00 & 21.7 \\
3B-2 & -2322.225399 & -2321.719610 & -2321.679317 & -2321.793222 & 0.27 & 13.8 \\
3B-3 & -2322.225521 & -2321.719655 & -2321.679438 & -2321.793093 & 0.35 & 12.1 \\
3B-4 & -2322.224458 & -2321.718849 & -2321.678502 & -2321.792921 & 0.46 & 10.1 \\
3B-5 & -2322.224817 & -2321.718958 & -2321.678748 & -2321.792312 & 0.84 & 5.3 \\
3B-6 & -2322.223145 & -2321.717520 & -2321.677139 & -2321.792078 & 0.99 & 4.1 \\
3B-7 & -2322.224009 & -2321.718305 & -2321.677981 & -2321.791993 & 1.04 & 3.8 \\
3B-8 & -2322.224410 & -2321.718515 & -2321.678277 & -2321.791886 & 1.11 & 3.4 \\
3B-9 & -2322.223909 & -2321.718220 & -2321.677873 & -2321.791708 & 1.22 & 2.8 \\
3B-10 & -2322.223964 & -2321.718082 & -2321.677812 & -2321.791691 & 1.23 & 2.7 \\
3B-11 & -2322.223583 & -2321.717886 & -2321.677524 & -2321.791665 & 1.24 & 2.7 \\
3B-12 & -2322.224136 & -2321.718205 & -2321.678008 & -2321.791437 & 1.39 & 2.1 \\
3B-13 & -2322.223310 & -2321.717641 & -2321.677292 & -2321.791328 & 1.46 & 1.9 \\
3B-14 & -2322.222783 & -2321.717040 & -2321.676691 & -2321.791272 & 1.49 & 1.8 \\
3B-15 & -2322.222669 & -2321.717006 & -2321.676621 & -2321.790873 & 1.74 & 1.1 \\
\hline
\end{tabular}

E: total energy; $E^{\prime}$ : total energy with zero point energy; H: enthalpy; G: Gibbs free energy; $\triangle$ G: relative Gibbs free energy at the B3LYP/6-31G(d,p) level in acetone $(P C M) . P_{G}$ : conformational distribution calculated from relative Gibbs free energy. 
Table S71. Important thermodynamic parameters and conformational analysis of $\mathbf{3 C}$ at the B3LYP/6-31G(d,p) level in acetone (PCM).

\begin{tabular}{clllllr}
\hline conformers & $E$ (a.u.) & $E^{\prime}$ (a.u.) & $H$ (a.u.) & $G$ (a.u.) & $\Delta G(\mathrm{kcal} / \mathrm{mol})$ & $\mathrm{P}_{\mathrm{G}}(\%)$ \\
\hline $\mathbf{3 C}-\mathbf{1}$ & -2322.231508 & -2321.725364 & -2321.685382 & -2321.798524 & 0.00 & 32.7 \\
$\mathbf{3 C}-2$ & -2322.230035 & -2321.724346 & -2321.684116 & -2321.797915 & 0.38 & 17.1 \\
$\mathbf{3 C}-\mathbf{3}$ & -2322.230079 & -2321.724259 & -2321.684102 & -2321.797860 & 0.42 & 16.2 \\
$\mathbf{3 C}-\mathbf{4}$ & -2322.229392 & -2321.723753 & -2321.683499 & -2321.797281 & 0.78 & 8.8 \\
$\mathbf{3 C}-\mathbf{5}$ & -2322.229136 & -2321.723582 & -2321.683295 & -2321.797016 & 0.95 & 6.6 \\
$\mathbf{3 C}-\mathbf{6}$ & -2322.228892 & -2321.722830 & -2321.682784 & -2321.796402 & 1.33 & 3.4 \\
$\mathbf{3 C}-\mathbf{7}$ & -2322.227459 & -2321.721792 & -2321.681513 & -2321.795823 & 1.69 & 1.9 \\
$\mathbf{3 C}-\mathbf{8}$ & -2322.228072 & -2321.721813 & -2321.681862 & -2321.795403 & 1.96 & 1.2 \\
$\mathbf{3 C - 9}$ & -2322.227475 & -2321.721679 & -2321.681497 & -2321.795400 & 1.96 & 1.2 \\
$\mathbf{3 C - 1 0}$ & -2322.228104 & -2321.722103 & -2321.682096 & -2321.795365 & 1.98 & 1.1 \\
\hline
\end{tabular}

E: total energy; $E$ ': total energy with zero point energy; H: enthalpy; G: Gibbs free energy; $\triangle G$ : relative Gibbs free energy at the B3LYP/6-31G(d,p) level in acetone $(P C M) . P_{G}$ : conformational distribution calculated from relative Gibbs free energy.

Table S72. Important thermodynamic parameters and conformational analysis of $3 D$ at the B3LYP/6-31G(d,p) level in acetone (PCM).

\begin{tabular}{cllllll}
\hline conformers & $E$ (a.u.) & $E^{\prime}($ a.u. $)$ & $H$ (a.u. $)$ & $G$ (a.u. $)$ & $\Delta G(\mathrm{kcal} / \mathrm{mol})$ & $\mathrm{P}_{\mathrm{G}}(\%)$ \\
\hline 3D-1 & -2322.227660 & -2321.722222 & -2321.681723 & -2321.796705 & 0.00 & 21.7 \\
3D-2 & -2322.227322 & -2321.721981 & -2321.681448 & -2321.796655 & 0.03 & 20.5 \\
3D-3 & -2322.228742 & -2321.722929 & -2321.682649 & -2321.796414 & 0.18 & 15.9 \\
3D-4 & -2322.228205 & -2321.722479 & -2321.682155 & -2321.796183 & 0.33 & 12.5 \\
3D-5 & -2322.227537 & -2321.721687 & -2321.681435 & -2321.795071 & 1.03 & 3.8 \\
3D-6 & -2322.226291 & -2321.720767 & -2321.680318 & -2321.795017 & 1.06 & 3.6 \\
3D-7 & -2322.227425 & -2321.721319 & -2321.681210 & -2321.794704 & 1.26 & 2.6 \\
3D-8 & -2322.225095 & -2321.719798 & -2321.679221 & -2321.794625 & 1.31 & 2.4 \\
3D-9 & -2322.226801 & -2321.721032 & -2321.680730 & -2321.794596 & 1.32 & 2.3 \\
3D-10 & -2322.225372 & -2321.720091 & -2321.679521 & -2321.794550 & 1.35 & 2.2 \\
3D-11 & -2322.226645 & -2321.720790 & -2321.680508 & -2321.794426 & 1.43 & 1.9 \\
3D-12 & -2322.226628 & -2321.720934 & -2321.680610 & -2321.794414 & 1.44 & 1.9 \\
3D-13 & -2322.225445 & -2321.719988 & -2321.679493 & -2321.794259 & 1.53 & 1.69 \\
3D-14 & -2322.226181 & -2321.720344 & -2321.680053 & -2321.794018 & 1.69 & 1.3 \\
3D-15 & -2322.226051 & -2321.720358 & -2321.680010 & -2321.793885 & 1.77 & 1.1 \\
\hline
\end{tabular}

E: total energy; $E$ ': total energy with zero point energy; H: enthalpy; G: Gibbs free energy; $\triangle$ G: relative Gibbs free energy at the B3LYP/6-3lG(d,p) level in acetone (PCM). $P_{G}$ : conformational distribution calculated from relative Gibbs free energy. 
Table S73. Calculated ${ }^{1} \mathrm{H}$ NMR chemical shifts of $\mathbf{3 A}$.

\begin{tabular}{|c|c|c|c|c|c|c|c|c|c|c|c|c|c|c|}
\hline \multirow[b]{2}{*}{ Position } & \multicolumn{13}{|c|}{ calculated $^{a}$} & \multirow{2}{*}{$\begin{array}{c}\text { experimental }^{b} \\
3\end{array}$} \\
\hline & 3A-1 & 3A-2 & 3A-3 & $3 A-4$ & $3 A-5$ & $3 \mathrm{~A}-6$ & 3A-7 & 3A-8 & 3A-9 & 3A-10 & 3A-11 & $\begin{array}{c}\text { averaged- } \\
\mathbf{3 A}^{c}\end{array}$ & $\begin{array}{l}\text { averaged-3A } \\
{\text { (corrected) }{ }^{d}}^{\text {cot }}\end{array}$ & \\
\hline 1 & 5.17 & 5.30 & 5.25 & 5.28 & 5.22 & 5.19 & 5.13 & 5.30 & 5.25 & $\begin{array}{l}5.19 \\
\end{array}$ & 5.28 & 5.13 & 4.89 & 5.17 \\
\hline 2 & 3.65 & 3.95 & 3.96 & 3.96 & 3.97 & 3.94 & 3.94 & 3.93 & 3.94 & 3.94 & 3.96 & 3.94 & 3.63 & 3.65 \\
\hline 3 & 3.65 & 3.73 & 3.72 & 4.02 & 4.01 & 3.86 & 3.84 & 3.72 & 3.71 & 3.87 & 4.02 & 3.85 & 3.51 & 3.65 \\
\hline 4 & 5.06 & 5.41 & 5.42 & 5.56 & 5.58 & 5.44 & 5.44 & 5.40 & 5.41 & 5.43 & 5.56 & 5.44 & 5.10 & 5.06 \\
\hline 5 & 4.23 & 4.37 & 4.35 & 4.30 & 4.30 & 4.33 & 4.32 & 4.38 & 4.37 & 4.35 & 4.31 & 4.33 & 4.01 & 4.23 \\
\hline \multirow[t]{2}{*}{6} & 4.04 & 4.26 & 4.26 & 4.25 & 4.26 & 4.27 & 4.27 & 4.27 & 4.26 & 4.27 & 4.26 & 4.28 & 3.93 & 4.04 \\
\hline & 5.12 & 5.56 & 5.58 & 5.69 & 5.72 & 5.58 & 5.60 & 5.57 & 5.59 & 5.59 & 5.69 & 5.61 & 5.24 & 5.12 \\
\hline $3^{\prime}, 5^{\prime}$ & 6.18 & 6.44 & 6.47 & 6.45 & 6.50 & 6.44 & 6.49 & 6.45 & 6.47 & 6.44 & 6.45 & 6.49 & 6.07 & 6.18 \\
\hline $8^{\prime}$ & 2.62 & 2.90 & 2.90 & 2.88 & 2.90 & 2.89 & 2.89 & 2.91 & 2.90 & 2.90 & 2.89 & 2.89 & 2.60 & 2.62 \\
\hline 3" & 7.18 & 7.89 & 7.90 & 7.74 & 7.75 & 7.88 & 7.89 & 7.89 & 7.90 & 7.89 & 7.74 & 7.89 & 7.42 & 7.18 \\
\hline 2"' & 4.64 & 5.11 & 5.10 & 5.11 & 5.11 & 5.12 & 5.11 & 5.15 & 5.14 & 5.16 & 5.15 & 5.15 & 4.76 & 4.64 \\
\hline 3"' & 3.44 & 3.90 & 3.90 & 3.92 & 3.92 & 3.90 & 3.90 & 4.10 & 4.09 & 4.10 & 4.11 & 4.10 & 3.61 & 3.44 \\
\hline \multirow[t]{2}{*}{$4^{\prime \prime \prime}$} & 1.93 & 2.28 & 2.28 & 2.31 & 2.33 & 2.30 & 2.31 & 2.29 & 2.29 & 2.32 & 2.33 & 2.32 & 2.02 & 1.93 \\
\hline & 2.14 & 2.60 & 2.60 & 2.57 & 2.59 & 2.58 & 2.59 & 2.21 & 2.21 & 2.21 & 2.23 & 2.22 & 2.25 & 2.14 \\
\hline
\end{tabular}

${ }^{a}$ Calculated using the GIAO method at the mPWIPW91/6-311+G(2d,p) level in acetone $(P C M) .{ }^{b}$ Measured in acetone-d ${ }_{6}(500 \mathrm{MHz})$. ${ }^{c}$ Averaged according to the Boltzmann

distribution theory at $298 \mathrm{~K}$ based on relative Gibbs free energies. ${ }^{d}$ Linearly corrected for the experimental data.

Table S74. Calculated ${ }^{1} \mathrm{H}$ NMR chemical shifts of $\mathbf{3 B}$.

\begin{tabular}{|c|c|c|c|c|c|c|c|c|c|c|c|c|c|c|c|c|c|c|}
\hline \multirow[b]{2}{*}{ Position } & \multicolumn{17}{|c|}{ calculated $^{a}$} & \multirow{2}{*}{$\begin{array}{c}\text { experimental }^{b} \\
3\end{array}$} \\
\hline & 3B-1 & 3B-2 & 3B-3 & 3B-4 & 3B-5 & 3B-6 & 3B-7 & 3B-8 & 3B-9 & 3B-10 & 3B-11 & 3B-12 & 3B-13 & 3B-14 & $3 B-15$ & $\begin{array}{c}\text { averaged- } \\
\mathbf{3 B}^{c}\end{array}$ & $\begin{array}{l}\text { averaged-3B } \\
\text { (corrected) }^{d}\end{array}$ & \\
\hline 1 & 5.39 & 5.34 & 5.28 & 5.51 & 5.49 & 5.40 & 5.46 & 5.38 & 5.34 & 5.33 & 5.29 & 5.46 & 5.48 & 5.37 & 5.44 & 5.39 & 5.12 & 5.17 \\
\hline 2 & 3.79 & 3.80 & 3.81 & 3.87 & 3.93 & 3.78 & 3.89 & 3.80 & 3.81 & 3.82 & 3.81 & 3.94 & 3.89 & 3.80 & 3.91 & 3.82 & 3.13 & 3.65 \\
\hline 3 & 5.55 & 5.54 & 5.25 & 5.48 & 5.19 & 5.54 & 5.47 & 5.25 & 5.56 & 5.25 & 5.55 & 5.18 & 5.16 & 5.53 & 5.15 & 5.43 & 5.17 & 3.65 \\
\hline 4 & 4.46 & 4.47 & 4.53 & 4.40 & 4.47 & 4.48 & 4.41 & 4.52 & 4.43 & 4.52 & 4.44 & 4.48 & 4.43 & 4.49 & 4.43 & 4.47 & 3.95 & 5.06 \\
\hline 5 & 5.54 & 5.54 & 5.31 & 5.53 & 5.35 & 5.55 & 5.54 & 5.46 & 5.51 & 5.47 & 5.51 & 5.37 & 5.48 & 5.55 & 5.49 & 5.48 & 5.24 & 4.23 \\
\hline \multirow[t]{2}{*}{6} & 3.96 & 3.98 & 5.46 & 3.94 & 5.39 & 4.00 & 3.96 & 5.39 & 4.07 & 5.42 & 4.10 & 5.41 & 5.36 & 4.02 & 5.39 & 4.44 & 3.91 & 4.04 \\
\hline & 5.09 & 5.08 & 4.18 & 5.09 & 4.19 & 5.17 & 5.08 & 4.23 & 5.09 & 4.22 & 5.09 & 4.19 & 4.23 & 5.16 & 4.23 & 4.81 & 4.38 & 5.12 \\
\hline $3^{\prime}, 5^{\prime}$ & 6.34 & 6.38 & 6.38 & 6.35 & 6.35 & 6.35 & 6.40 & 6.31 & 6.37 & 6.40 & 6.39 & 6.38 & 6.33 & 6.40 & 6.40 & 6.36 & 6.35 & 6.18 \\
\hline $8^{\prime}$ & 2.87 & 2.86 & 2.89 & 2.87 & 2.89 & 2.87 & 2.86 & 2.87 & 2.90 & 2.85 & 2.89 & 2.87 & 2.88 & 2.86 & 2.84 & 2.87 & 1.93 & 2.62 \\
\hline $3^{\prime \prime}$ & 7.86 & 7.86 & 7.95 & 7.84 & 7.94 & 7.88 & 7.84 & 7.97 & 7.80 & 7.97 & 7.80 & 7.94 & 7.95 & 7.88 & 7.94 & 7.88 & 8.28 & 7.18 \\
\hline $2^{\prime \prime \prime}$ & 4.39 & 4.40 & 4.61 & 4.38 & 4.59 & 4.36 & 4.40 & 4.48 & 4.42 & 4.48 & 4.43 & 4.60 & 4.46 & 4.38 & 4.47 & 4.45 & 3.92 & 4.64 \\
\hline 3"' & 2.87 & 2.86 & 3.23 & 2.86 & 3.22 & 3.13 & 2.86 & 3.01 & 3.22 & 3.01 & 3.22 & 3.22 & 2.99 & 3.14 & 2.99 & 3.00 & 2.08 & 3.44 \\
\hline \multirow[t]{2}{*}{$4^{\prime \prime \prime}$} & 3.48 & 3.48 & 2.85 & 3.46 & 2.85 & 3.22 & 3.46 & 3.77 & 2.96 & 3.78 & 2.96 & 2.85 & 3.76 & 3.22 & 3.78 & 3.32 & 2.49 & 1.93 \\
\hline & 3.95 & 3.95 & 3.82 & 3.95 & 3.81 & 4.00 & 3.95 & 2.91 & 3.54 & 2.92 & 3.55 & 3.82 & 2.90 & 4.01 & 2.91 & 3.79 & 3.09 & 2.14 \\
\hline
\end{tabular}

${ }^{a}$ Calculated using the GIAO method at the mPWIPW91/6-311+G(2d,p) level in acetone $(P C M) .{ }^{b}$ Measured in acetone- ${ }_{6}(500 \mathrm{MHz}) .{ }^{c}$ Averaged according to the Boltzmann distribution theory at $298 \mathrm{~K}$ based on relative Gibbs free energies. ${ }^{d}$ Linearly corrected for the experimental data. 
Table S75. Calculated ${ }^{1} \mathrm{H}$ NMR chemical shifts of $\mathbf{3 C}$.

\begin{tabular}{|c|c|c|c|c|c|c|c|c|c|c|c|c|c|}
\hline \multirow[b]{2}{*}{ Position } & \multicolumn{12}{|c|}{ calculated $^{a}$} & \multirow{2}{*}{$\begin{array}{c}\text { experimental }^{b} \\
3\end{array}$} \\
\hline & 3C-1 & $3 C-2$ & $3 C-3$ & $3 C-4$ & 3C-5 & 3C-6 & 3C-7 & $3 C-8$ & 3C-9 & 3C-10 & $\begin{array}{c}\text { averaged- } \\
\mathbf{3 C}^{c}\end{array}$ & $\begin{array}{c}\text { averaged-3C } \\
{\text { (corrected) }{ }^{d}}^{\text {corecte }}\end{array}$ & \\
\hline 1 & 5.30 & 5.24 & 5.19 & 5.18 & 5.30 & 5.31 & 5.25 & 5.25 & 5.19 & 5.52 & 5.26 & 4.97 & 5.17 \\
\hline 2 & 3.98 & 3.95 & 3.96 & 3.93 & 3.97 & 3.97 & 3.95 & 3.87 & 3.96 & 3.91 & 3.96 & 3.52 & 3.65 \\
\hline 3 & 3.74 & 4.06 & 3.86 & 4.04 & 4.06 & 3.75 & 4.07 & 3.75 & 3.86 & 3.77 & 3.88 & 3.44 & 3.65 \\
\hline 4 & 5.44 & 5.67 & 5.46 & 5.69 & 5.60 & 5.45 & 5.69 & 5.52 & 5.47 & 5.46 & 5.53 & 5.28 & 5.06 \\
\hline 5 & 4.26 & 4.20 & 4.23 & 4.20 & 4.27 & 4.28 & 4.23 & 4.27 & 4.26 & 4.16 & 4.24 & 3.83 & 4.23 \\
\hline \multirow[t]{2}{*}{6} & 4.14 & 4.10 & 4.14 & 4.10 & 4.18 & 4.18 & 4.17 & 4.20 & 4.19 & 4.06 & 4.13 & 3.71 & 4.04 \\
\hline & 5.50 & 5.59 & 5.52 & 5.62 & 5.65 & 5.52 & 5.61 & 5.59 & 5.55 & 5.50 & 5.55 & 5.30 & 5.12 \\
\hline $3^{\prime}, 5^{\prime}$ & 6.42 & 6.41 & 6.42 & 6.44 & 6.42 & 6.43 & 6.42 & 6.49 & 6.42 & 6.45 & 6.42 & 6.28 & 6.18 \\
\hline $8^{\prime}$ & 2.88 & 2.90 & 2.89 & 2.91 & 2.92 & 2.88 & 2.90 & 2.92 & 2.89 & 2.93 & 2.89 & 2.32 & 2.62 \\
\hline 3" & 8.02 & 7.89 & 8.02 & 7.89 & 7.79 & 8.03 & 7.90 & 8.16 & 8.03 & 8.01 & 7.97 & 8.01 & 7.18 \\
\hline 2"' & 4.44 & 4.43 & 4.43 & 4.43 & 4.45 & 4.44 & 4.43 & 6.10 & 4.43 & 4.33 & 4.45 & 4.08 & 4.64 \\
\hline 3"' & 2.80 & 2.84 & 2.80 & 2.85 & 3.23 & 3.04 & 3.06 & 2.76 & 3.05 & 2.75 & 2.86 & 2.29 & 3.44 \\
\hline \multirow[t]{2}{*}{ 4"' } & 3.55 & 3.51 & 3.54 & 3.50 & 2.92 & 3.29 & 3.30 & 3.45 & 3.30 & 3.41 & 3.47 & 2.97 & 1.93 \\
\hline & 3.47 & 3.61 & 3.51 & 3.62 & 3.59 & 3.43 & 3.57 & 3.05 & 3.47 & 3.43 & 3.52 & 3.03 & 2.14 \\
\hline
\end{tabular}

${ }^{a}$ Calculated using the GIAO method at the mPWIPW91/6-311+G(2d,p) level in acetone $(P C M) .{ }^{b}$ Measured in acetone-d $6(500 \mathrm{MHz}) .{ }^{c}$ Averaged according to the

Boltzmann distribution theory at $298 \mathrm{~K}$ based on relative Gibbs free energies. ${ }^{d}$ Linearly corrected for the experimental data.

Table S76. Calculated ${ }^{1} \mathrm{H}$ NMR chemical shifts of 3D.

\begin{tabular}{|c|c|c|c|c|c|c|c|c|c|c|c|c|c|c|c|c|c|c|}
\hline \multirow[b]{2}{*}{ Position } & \multicolumn{17}{|c|}{ calculated $^{a}$} & \multirow{2}{*}{$\begin{array}{c}\text { experimental }^{b} \\
3\end{array}$} \\
\hline & 3D-1 & 3D-2 & 3D-3 & $3 D-4$ & 3D-5 & 3D-6 & 3D-7 & 3D-8 & 3D-9 & 3D-10 & 3D-11 & 3D-12 & 3D-13 & 3D-14 & 3D-15 & $\begin{array}{c}\text { averaged- } \\
\mathbf{3 D}^{c}\end{array}$ & $\begin{array}{l}\text { averaged-3D } \\
{\text { (corrected) }{ }^{d}}^{d}\end{array}$ & \\
\hline 1 & njhbvg & 5.36 & 5.32 & 5.29 & 5.46 & 5.53 & 5.42 & 5.34 & 5.46 & 5.44 & 5.32 & 5.34 & 5.41 & 5.29 & 5.33 & 5.37 & 5.01 & 5.17 \\
\hline 2 & 3.74 & 3.75 & 3.75 & 3.75 & 3.87 & 3.80 & 3.72 & 3.75 & 3.87 & 3.83 & 3.75 & 3.92 & 3.74 & 3.75 & 3.91 & 3.76 & 3.28 & 3.65 \\
\hline 3 & 5.59 & 5.58 & 5.20 & 5.21 & 5.15 & 5.48 & 4.95 & 5.59 & 5.16 & 5.48 & 5.22 & 5.13 & 5.60 & 5.22 & 5.15 & 5.39 & 5.03 & 3.65 \\
\hline 4 & 4.43 & 4.44 & 4.51 & 4.53 & 4.49 & 4.36 & 4.35 & 4.42 & 4.51 & 4.39 & 4.52 & 4.48 & 4.42 & 4.54 & 4.51 & 4.46 & 4.03 & 5.06 \\
\hline 5 & 5.59 & 5.58 & 5.33 & 5.35 & 5.38 & 5.58 & 5.31 & 5.53 & 5.40 & 5.63 & 5.32 & 5.40 & 5.55 & 5.34 & 5.42 & 5.47 & 5.12 & 4.23 \\
\hline \multirow[t]{2}{*}{6} & 4.08 & 4.10 & 5.40 & 5.44 & 5.39 & 4.05 & 5.38 & 4.11 & 5.41 & 4.06 & 5.42 & 5.38 & 4.09 & 5.45 & 5.42 & 4.70 & 4.29 & 4.04 \\
\hline & 5.13 & 5.12 & 4.22 & 4.22 & 4.23 & 5.10 & 4.24 & 5.16 & 4.23 & 5.10 & 4.28 & 4.22 & 5.17 & 4.27 & 4.22 & 4.71 & 4.29 & 5.12 \\
\hline $3^{\prime}, 5^{\prime}$ & 6.45 & 6.48 & 6.48 & 6.50 & 6.47 & 6.44 & 6.46 & 6.48 & 6.48 & 6.45 & 6.48 & 6.49 & 6.45 & 6.50 & 6.52 & 6.47 & 6.19 & 6.18 \\
\hline $8^{\prime}$ & 2.90 & 2.90 & 2.91 & 2.92 & 2.91 & 2.89 & 2.91 & 2.90 & 2.92 & 2.90 & 2.91 & 2.90 & 2.90 & 2.92 & 2.91 & 2.90 & 2.36 & 2.62 \\
\hline $3^{\prime \prime}$ & 7.71 & 7.70 & 7.92 & 7.92 & 7.91 & 7.67 & 7.92 & 7.69 & 7.90 & 7.68 & 7.94 & 7.91 & 7.70 & 7.93 & 7.91 & 7.80 & 7.62 & 7.18 \\
\hline $2^{\prime \prime \prime}$ & 4.99 & 4.99 & 4.92 & 4.92 & 4.89 & 4.96 & 4.91 & 5.05 & 4.89 & 4.96 & 4.98 & 4.89 & 5.04 & 4.98 & 4.90 & 4.95 & 4.56 & 4.64 \\
\hline 3"' & 3.91 & 3.90 & 4.02 & 4.03 & 4.01 & 3.88 & 4.03 & 4.14 & 4.02 & 3.87 & 4.18 & 4.00 & 4.13 & 4.18 & 4.01 & 3.97 & 3.51 & 3.44 \\
\hline \multirow[t]{2}{*}{$4^{\prime \prime \prime}$} & 2.35 & 2.35 & 2.25 & 2.24 & 2.24 & 2.35 & 2.25 & 2.32 & 2.23 & 2.35 & 2.26 & 2.23 & 2.32 & 2.26 & 2.22 & 2.30 & 1.71 & 1.93 \\
\hline & 2.60 & 2.58 & 2.67 & 2.68 & 2.65 & 2.57 & 2.68 & 2.22 & 2.65 & 2.55 & 2.40 & 2.65 & 2.23 & 2.41 & 2.65 & 2.60 & 2.04 & 2.14 \\
\hline
\end{tabular}

${ }^{a}$ Calculated using the GIAO method at the mPWIPW91/6-311+G(2d,p) level in acetone $(P C M) .{ }^{b}$ Measured in acetone- $d_{6}(500 \mathrm{MHz}) .{ }^{c}$ Averaged according to the Boltzmann distribution theory at $298 \mathrm{~K}$ based on relative Gibbs free energies. ${ }^{d}$ Linearly corrected for the experimental data. 
Table S77. Calculated ${ }^{13} \mathrm{C}$ NMR chemical shifts of $\mathbf{3 A}$.

\begin{tabular}{|c|c|c|c|c|c|c|c|c|c|c|c|c|c|c|}
\hline \multirow[b]{2}{*}{ Position } & \multicolumn{13}{|c|}{ calculated $^{a}$} & \multirow{2}{*}{$\frac{\text { experimental }^{b}}{3}$} \\
\hline & 3A-1 & 3A-2 & 3A-3 & $3 \mathrm{~A}-4$ & $3 A-5$ & $3 A-6$ & 3A-7 & 3A-8 & 3A-9 & 3A-10 & 3A-11 & averaged $-3 A^{c}$ & $\begin{array}{c}\text { averaged-3A } \text { (corrected) }^{d} \\
\end{array}$ & \\
\hline 1 & 98.4 & 98.5 & 98.9 & 99.4 & 99.4 & 99.8 & 98.6 & 98.8 & 99.5 & 98.9 & 99.9 & 98.9 & 98.8 & 100.9 \\
\hline 2 & 73.0 & 73.0 & 73.4 & 73.4 & 74.0 & 74.1 & 72.9 & 72.9 & 73.9 & 73.3 & 73.9 & 73.3 & 74.2 & 74.5 \\
\hline 3 & 76.5 & 76.5 & 73.9 & 73.8 & 75.1 & 75.0 & 76.6 & 76.6 & 75.2 & 74.0 & 75.1 & 75.5 & 76.3 & 75.9 \\
\hline 4 & 75.5 & 75.4 & 73.5 & 73.4 & 76.5 & 76.3 & 75.6 & 75.5 & 76.6 & 73.6 & 76.4 & 75.1 & 76.0 & 76.2 \\
\hline 5 & 68.9 & 68.9 & 69.2 & 69.3 & 68.6 & 68.7 & 68.7 & 68.7 & 68.4 & 69.0 & 68.5 & 68.9 & 70.0 & 70.4 \\
\hline 6 & 61.9 & 61.9 & 62.0 & 62.1 & 61.9 & 61.9 & 62.0 & 62.0 & 62.0 & 62.1 & 62.0 & 61.9 & 63.3 & 64.1 \\
\hline $1^{\prime}$ & 105.3 & 106.1 & 105.6 & 106.5 & 105.5 & 106.6 & 105.1 & 105.6 & 105.3 & 105.5 & 106.4 & 105.8 & 105.4 & 106.7 \\
\hline $2^{\prime}, 6^{\prime}$ & 167.2 & 167.0 & 167.0 & 166.8 & 167.1 & 166.9 & 167.2 & 167.1 & 167.2 & 167.0 & 166.9 & 167.1 & 164.4 & 164.9 \\
\hline $3^{\prime}, 5^{\prime}$ & 94.1 & 94.2 & 94.4 & 94.3 & 94.2 & 94.2 & 93.9 & 94.1 & 94.1 & 94.3 & 94.2 & 94.2 & 94.3 & 95.9 \\
\hline $4^{\prime}$ & 165.9 & 166.3 & 166.1 & 166.9 & 166.0 & 166.8 & 165.9 & 166.2 & 166.1 & 166.0 & 166.8 & 166.2 & 163.5 & 164.3 \\
\hline $7^{\prime}$ & 207.9 & 207.8 & 208.2 & 208.8 & 208.1 & 208.5 & 207.8 & 207.5 & 208.1 & 208.2 & 208.3 & 208.1 & 203.8 & 204.4 \\
\hline $8^{\prime}$ & 30.3 & 30.7 & 30.5 & 31.0 & 30.4 & 30.9 & 30.2 & 30.7 & 30.2 & 30.4 & 30.9 & 30.5 & 33.1 & 32.9 \\
\hline 1" & 117.0 & 117.0 & 115.7 & 115.6 & 116.6 & 116.6 & 116.8 & 116.8 & 116.4 & 115.5 & 116.4 & 116.5 & 115.8 & 115.6 \\
\hline $2^{\prime \prime}$ & 120.3 & 120.3 & 122.5 & 122.4 & 120.6 & 120.6 & 120.2 & 120.2 & 120.5 & 122.4 & 120.5 & 120.9 & 120.0 & 120.0 \\
\hline 3" & 116.6 & 116.6 & 115.3 & 115.3 & 116.6 & 116.6 & 116.8 & 116.9 & 116.8 & 115.4 & 116.8 & 116.3 & 115.5 & 114.3 \\
\hline 4" & 147.9 & 147.9 & 147.9 & 147.9 & 147.9 & 147.9 & 148.1 & 148.1 & 148.0 & 148.0 & 148.1 & 147.9 & 146.0 & 147.5 \\
\hline 5" & 134.8 & 134.8 & 132.8 & 132.9 & 134.4 & 134.5 & 135.2 & 135.4 & 134.9 & 133.1 & 134.9 & 134.3 & 132.8 & 134.9 \\
\hline 6" & 142.3 & 142.4 & 141.6 & 141.6 & 142.1 & 142.1 & 142.9 & 143.0 & 142.6 & 142.0 & 142.6 & 142.2 & 140.4 & 142.6 \\
\hline 7" & 173.1 & 173.0 & 169.9 & 169.8 & 173.5 & 173.3 & 173.2 & 173.2 & 173.5 & 170.0 & 173.4 & 172.3 & 169.4 & 165.6 \\
\hline 1"' & 179.6 & 179.6 & 179.9 & 179.9 & 179.6 & 179.6 & 179.6 & 179.6 & 179.6 & 179.9 & 179.6 & 179.7 & 176.5 & 175.6 \\
\hline $2^{\prime \prime \prime}$ & 47.7 & 47.7 & 47.5 & 47.6 & 47.7 & 47.7 & 47.9 & 47.9 & 47.9 & 47.9 & 47.9 & 47.7 & 49.6 & 46.9 \\
\hline 3"' & 43.1 & 43.1 & 43.1 & 43.1 & 43.1 & 43.1 & 44.0 & 43.9 & 44.1 & 44.1 & 44.0 & 43.2 & 45.3 & 43.6 \\
\hline 4"' & 27.2 & 27.2 & 27.4 & 27.4 & 27.3 & 27.3 & 29.0 & 29.0 & 28.9 & 29.0 & 28.9 & 27.5 & 30.2 & 30.4 \\
\hline $5^{\prime \prime \prime}$ & 176.8 & 176.8 & 176.9 & 176.9 & 176.8 & 176.8 & 174.4 & 174.4 & 174.4 & 174.6 & 174.4 & 176.5 & 173.4 & 172.2 \\
\hline $6^{\prime \prime \prime}$ & 177.6 & 177.5 & 177.2 & 177.1 & 177.5 & 177.4 & 177.6 & 177.6 & 177.6 & 177.4 & 177.5 & 177.4 & 174.3 & 172.1 \\
\hline
\end{tabular}

the Boltzmann distribution theory at $298 \mathrm{~K}$ based on relative Gibbs free energies. ${ }^{d}$ Linearly corrected for the experimental data. 
Table S78. Calculated ${ }^{13} \mathrm{C}$ NMR chemical shifts of 3B.

\begin{tabular}{|c|c|c|c|c|c|c|c|c|c|c|c|c|c|c|c|c|c|c|}
\hline \multirow[b]{2}{*}{ Position } & \multicolumn{17}{|c|}{ calculated $^{a}$} & \multirow{2}{*}{$\frac{\text { experimental }^{b}}{3}$} \\
\hline & 3B-1 & 3B-2 & 3B-3 & $3 B-4$ & 3B-5 & 3B-6 & 3B-7 & 3B-8 & 3B-9 & 3B-10 & 3B-11 & 3B-12 & 3B-13 & 3B-14 & 3B-15 & averaged-3 $\mathbf{3}{ }^{c}$ & $\begin{array}{l}\text { averaged-3B } \\
{\text { (corrected) }{ }^{d}}^{-1}\end{array}$ & \\
\hline 1 & 97.5 & 97.7 & 98.6 & 97.0 & 97.8 & 97.4 & 97.2 & 98.2 & 97.5 & 98.6 & 97.8 & 98.0 & 97.8 & 97.5 & 98.1 & 97.7 & 97.8 & 100.9 \\
\hline 2 & 73.6 & 73.6 & 74.2 & 71.3 & 71.8 & 73.4 & 71.3 & 74.2 & 73.8 & 74.2 & 73.8 & 71.8 & 71.9 & 73.4 & 71.9 & 73.2 & 74.1 & 74.5 \\
\hline 3 & 68.0 & 68.0 & 67.7 & 70.7 & 70.5 & 68.0 & 70.7 & 68.1 & 68.0 & 68.1 & 68.0 & 70.5 & 70.9 & 67.9 & 70.9 & 68.7 & 69.8 & 75.9 \\
\hline 4 & 79.0 & 79.0 & 80.5 & 80.7 & 82.3 & 78.9 & 80.7 & 80.0 & 79.6 & 80.0 & 79.6 & 82.2 & 81.6 & 78.9 & 81.6 & 79.9 & 80.6 & 76.2 \\
\hline 5 & 63.8 & 63.7 & 64.3 & 63.6 & 63.9 & 63.9 & 63.5 & 63.7 & 63.5 & 63.7 & 63.4 & 63.9 & 63.4 & 63.8 & 63.3 & 63.8 & 65.0 & 70.4 \\
\hline 6 & 64.5 & 64.5 & 62.1 & 64.6 & 62.2 & 64.5 & 64.5 & 62.0 & 65.4 & 61.9 & 65.4 & 62.2 & 62.1 & 64.5 & 62.0 & 63.8 & 65.1 & 64.1 \\
\hline $1^{\prime}$ & 105.6 & 105.8 & 106.1 & 105.4 & 105.0 & 105.5 & 105.7 & 105.3 & 105.2 & 105.7 & 106.2 & 105.9 & 105.1 & 105.7 & 105.7 & 105.6 & 105.4 & 106.7 \\
\hline $2^{\prime}, 6^{\prime}$ & 166.9 & 167.0 & 166.6 & 166.9 & 166.8 & 166.9 & 167.1 & 167.0 & 166.8 & 167.1 & 166.8 & 166.7 & 167.0 & 167.1 & 167.2 & 166.9 & 164.4 & 164.9 \\
\hline $3^{\prime}, 5^{\prime}$ & 94.1 & 94.3 & 94.2 & 93.9 & 94.0 & 93.9 & 94.2 & 93.7 & 94.3 & 94.0 & 94.3 & 94.0 & 93.6 & 94.1 & 93.9 & 94.1 & 94.3 & 95.9 \\
\hline $4^{\prime}$ & 166.3 & 166.7 & 166.7 & 166.1 & 166.4 & 166.1 & 166.6 & 166.1 & 166.4 & 166.6 & 166.6 & 166.7 & 166.1 & 166.5 & 166.5 & 166.4 & 164.0 & 164.3 \\
\hline $7^{\prime}$ & 207.9 & 208.8 & 208.9 & 207.7 & 207.8 & 207.8 & 208.5 & 207.7 & 208.0 & 208.8 & 208.6 & 208.7 & 207.6 & 208.7 & 208.5 & 208.2 & 204.3 & 204.4 \\
\hline $8^{\prime}$ & 30.3 & 30.7 & 31.0 & 30.2 & 29.9 & 30.3 & 30.6 & 30.2 & 30.1 & 30.7 & 31.0 & 30.9 & 30.1 & 30.6 & 30.6 & 30.5 & 32.9 & 32.9 \\
\hline 1" & 119.1 & 119.1 & 122.6 & 119.3 & 122.8 & 118.8 & 119.3 & 122.0 & 120.4 & 122.0 & 120.3 & 122.8 & 122.2 & 118.8 & 122.2 & 120.3 & 119.5 & 115.6 \\
\hline 2" & 122.6 & 122.6 & 120.5 & 122.2 & 120.2 & 122.5 & 122.2 & 120.6 & 121.9 & 120.6 & 121.9 & 120.2 & 120.2 & 122.5 & 120.3 & 121.8 & 121.0 & 120.0 \\
\hline 3" & 114.6 & 114.5 & 115.0 & 114.5 & 115.1 & 114.8 & 114.5 & 115.2 & 114.0 & 115.1 & 114.0 & 115.1 & 115.2 & 114.8 & 115.2 & 114.7 & 114.1 & 114.3 \\
\hline 4" & 147.5 & 147.5 & 146.5 & 147.5 & 146.6 & 147.6 & 147.5 & 147.0 & 147.4 & 147.0 & 147.4 & 146.6 & 147.1 & 147.5 & 147.0 & 147.2 & 145.5 & 147.5 \\
\hline 5" & 133.8 & 133.9 & 133.3 & 134.1 & 133.7 & 133.9 & 134.1 & 134.1 & 133.9 & 134.0 & 133.8 & 133.6 & 134.3 & 133.9 & 134.3 & 133.8 & 132.6 & 134.9 \\
\hline 6" & 142.4 & 142.4 & 141.4 & 142.6 & 141.6 & 142.4 & 142.5 & 142.3 & 142.3 & 142.2 & 142.2 & 141.6 & 142.4 & 142.4 & 142.4 & 142.2 & 140.7 & 142.6 \\
\hline 7" & 169.2 & 169.2 & 169.9 & 169.3 & 170.1 & 169.0 & 169.4 & 169.8 & 169.5 & 169.8 & 169.5 & 170.2 & 170.0 & 169.1 & 170.0 & 169.4 & 166.9 & 165.6 \\
\hline $1^{\prime \prime \prime}$ & 178.9 & 178.9 & 179.5 & 178.8 & 179.4 & 179.0 & 178.8 & 178.4 & 179.2 & 178.4 & 179.1 & 179.4 & 178.3 & 178.9 & 178.3 & 179.0 & 176.1 & 175.6 \\
\hline $2^{\prime \prime \prime}$ & 46.2 & 46.2 & 45.9 & 46.3 & 45.9 & 46.6 & 46.3 & 47.6 & 46.7 & 47.6 & 46.7 & 45.9 & 47.6 & 46.6 & 47.6 & 46.4 & 48.3 & 46.9 \\
\hline $3^{\prime \prime \prime}$ & 44.9 & 45.0 & 44.1 & 44.9 & 44.0 & 45.2 & 45.0 & 43.5 & 44.6 & 43.6 & 44.6 & 44.1 & 43.5 & 45.3 & 43.6 & 44.6 & 46.6 & 43.6 \\
\hline 4"' & 30.6 & 30.6 & 31.1 & 30.6 & 31.1 & 32.3 & 30.6 & 33.8 & 30.1 & 33.9 & 30.1 & 31.1 & 33.8 & 32.3 & 33.8 & 31.1 & 33.6 & 30.4 \\
\hline $5^{\prime \prime \prime}$ & 177.8 & 177.8 & 177.8 & 177.8 & 177.7 & 175.3 & 177.8 & 177.0 & 176.8 & 177.0 & 176.8 & 177.8 & 176.9 & 175.3 & 177.0 & 177.5 & 174.7 & 172.2 \\
\hline 6"' & 176.2 & 176.3 & 181.1 & 176.1 & 181.0 & 175.8 & 176.2 & 182.2 & 176.7 & 182.2 & 176.8 & 181.0 & 182.1 & 175.9 & 182.0 & 177.9 & 175.0 & 172.1 \\
\hline
\end{tabular}

列

$298 \mathrm{~K}$ based on relative Gibbs free energies. ${ }^{d}$ Linearly corrected for the experimental data. 
Table S79. Calculated ${ }^{13} \mathrm{C}$ NMR chemical shifts of $\mathbf{3 C}$.

\begin{tabular}{|c|c|c|c|c|c|c|c|c|c|c|c|c|c|}
\hline \multirow[b]{2}{*}{ Position } & \multicolumn{12}{|c|}{ calculated $^{a}$} & \multirow{2}{*}{$\frac{\text { experimental }^{b}}{3}$} \\
\hline & 3C-1 & $3 \mathrm{C}-2$ & $3 \mathrm{C}-3$ & $3 C-4$ & $3 C-5$ & $3 \mathrm{C}-6$ & 3C-7 & 3C-8 & 3C-9 & $3 \mathrm{C}-10$ & averaged-3 $\mathbf{3} C^{c}$ & $\begin{array}{l}\text { averaged-3C } \\
\text { (corrected) }^{d}\end{array}$ & \\
\hline 1 & 98.1 & 99.1 & 99.4 & 99.8 & 98.8 & 98.2 & 99.1 & 98.9 & 99.5 & 101.4 & 98.8 & 98.6 & 100.9 \\
\hline 2 & 73.1 & 73.6 & 74.2 & 73.6 & 73.5 & 73.1 & 73.6 & 73.3 & 74.2 & 74.7 & 73.5 & 74.1 & 74.5 \\
\hline 3 & 75.4 & 73.0 & 74.2 & 72.9 & 73.4 & 75.4 & 73.0 & 75.2 & 74.2 & 75.1 & 74.3 & 74.8 & 75.9 \\
\hline 4 & 75.4 & 73.0 & 76.3 & 72.9 & 73.3 & 75.4 & 73.1 & 75.3 & 76.2 & 75.7 & 74.7 & 75.2 & 76.2 \\
\hline 5 & 68.9 & 69.6 & 68.8 & 69.7 & 68.9 & 68.9 & 69.6 & 69.0 & 68.8 & 69.8 & 69.1 & 69.8 & 70.4 \\
\hline 6 & 61.8 & 61.6 & 61.7 & 61.7 & 61.8 & 62.0 & 62.0 & 62.2 & 62.1 & 61.9 & 61.8 & 62.7 & 64.1 \\
\hline $1^{\prime}$ & 105.3 & 105.4 & 105.4 & 105.8 & 105.1 & 105.3 & 105.4 & 106.0 & 105.5 & 104.8 & 105.4 & 104.9 & 106.7 \\
\hline $2^{\prime}, 6^{\prime}$ & 166.9 & 166.9 & 166.9 & 167.2 & 167.0 & 166.9 & 166.9 & 167.2 & 166.9 & 167.0 & 166.9 & 164.5 & 164.9 \\
\hline $3^{\prime}, 5^{\prime}$ & 94.3 & 94.1 & 94.2 & 94.2 & 93.9 & 94.3 & 94.1 & 93.8 & 94.2 & 95.3 & 94.2 & 94.1 & 95.9 \\
\hline $4^{\prime}$ & 166.5 & 166.4 & 166.6 & 166.7 & 166.0 & 166.6 & 166.4 & 166.0 & 166.6 & 167.0 & 166.5 & 164.1 & 164.3 \\
\hline $7^{\prime}$ & 208.1 & 208.0 & 208.1 & 208.7 & 207.7 & 208.1 & 208.0 & 208.3 & 208.2 & 207.8 & 208.1 & 204.3 & 204.4 \\
\hline $8^{\prime}$ & 30.6 & 30.5 & 30.6 & 30.9 & 30.3 & 30.6 & 30.6 & 30.8 & 30.6 & 30.2 & 30.6 & 32.5 & 32.9 \\
\hline 1" & 120.5 & 119.3 & 120.4 & 119.2 & 119.4 & 120.3 & 119.2 & 120.3 & 120.2 & 119.6 & 120.0 & 119.0 & 115.6 \\
\hline $2^{\prime \prime}$ & 120.1 & 122.4 & 120.4 & 122.3 & 121.7 & 119.9 & 122.1 & 119.8 & 120.2 & 120.9 & 121.0 & 120.0 & 120.0 \\
\hline 3" & 116.6 & 115.5 & 116.7 & 115.6 & 115.0 & 116.7 & 115.6 & 116.2 & 116.7 & 116.5 & 116.2 & 115.4 & 114.3 \\
\hline 4" & 147.8 & 147.8 & 147.8 & 147.8 & 147.6 & 147.9 & 147.9 & 147.1 & 147.9 & 147.6 & 147.8 & 146.0 & 147.5 \\
\hline 5" & 135.0 & 133.8 & 134.9 & 133.8 & 133.9 & 135.1 & 133.8 & 133.5 & 135.0 & 134.6 & 134.5 & 133.1 & 134.9 \\
\hline 6" & 142.5 & 142.5 & 142.5 & 142.5 & 142.4 & 142.6 & 142.6 & 142.0 & 142.6 & 142.2 & 142.5 & 140.8 & 142.6 \\
\hline 7" & 172.7 & 169.4 & 172.8 & 169.4 & 169.6 & 172.5 & 169.4 & 172.5 & 172.6 & 172.9 & 171.5 & 168.9 & 165.6 \\
\hline 1"' & 178.2 & 178.5 & 178.2 & 178.6 & 179.0 & 177.9 & 178.2 & 178.7 & 177.9 & 178.2 & 178.3 & 175.5 & 175.6 \\
\hline $2^{\prime \prime \prime}$ & 46.6 & 46.7 & 46.7 & 46.8 & 46.0 & 46.7 & 46.9 & 44.3 & 46.8 & 45.5 & 46.6 & 48.0 & 46.9 \\
\hline 3"' & 45.0 & 45.0 & 45.0 & 45.0 & 45.2 & 45.3 & 45.3 & 45.2 & 45.3 & 45.1 & 45.1 & 46.5 & 43.6 \\
\hline 4"' & 31.0 & 30.3 & 30.8 & 30.3 & 29.0 & 32.8 & 32.1 & 27.7 & 32.5 & 31.4 & 30.7 & 32.6 & 30.4 \\
\hline $5^{\prime \prime \prime}$ & 177.4 & 177.5 & 177.4 & 177.5 & 177.1 & 174.9 & 175.2 & 177.5 & 175.0 & 177.3 & 177.3 & 174.5 & 172.2 \\
\hline $6^{\prime \prime \prime}$ & 175.6 & 174.9 & 175.3 & 174.8 & 175.9 & 175.3 & 174.8 & 175.0 & 175.1 & 175.7 & 175.3 & 172.6 & 172.1 \\
\hline
\end{tabular}


Table S80. Calculated ${ }^{13} \mathrm{C}$ NMR chemical shifts of $3 D$.

\begin{tabular}{|c|c|c|c|c|c|c|c|c|c|c|c|c|c|c|c|c|c|c|}
\hline \multirow[b]{2}{*}{ Position } & \multicolumn{17}{|c|}{ calculated $^{a}$} & \multirow{2}{*}{$\begin{array}{c}\text { experimental }^{b} \\
3 \\
\end{array}$} \\
\hline & 3D-1 & 3D-2 & 3D-3 & 3D-4 & 3D-5 & 3D-6 & 3D-7 & 3D-8 & 3D-9 & 3D-10 & 3D-11 & 3D-12 & 3D-13 & 3D-14 & 3D-15 & averaged-3D ${ }^{c}$ & $\begin{array}{c}\text { averaged-3D } \\
\text { (corrected) }^{d}\end{array}$ & \\
\hline 1 & 97.8 & 98.0 & 97.9 & 98.4 & 97.6 & 97.3 & 97.9 & 98.0 & 97.9 & 98.3 & 97.9 & 98.7 & 97.7 & 98.4 & 99.2 & 98.0 & 98.3 & 100.9 \\
\hline 2 & 73.2 & 73.2 & 73.8 & 73.8 & 71.6 & 71.0 & 73.5 & 73.3 & 71.6 & 71.4 & 73.9 & 72.1 & 73.3 & 73.8 & 72.1 & 73.2 & 74.4 & 74.5 \\
\hline 3 & 67.6 & 67.6 & 67.4 & 67.4 & 70.3 & 70.3 & 70.3 & 67.5 & 70.4 & 69.0 & 67.3 & 68.7 & 67.5 & 67.3 & 68.7 & 68.0 & 69.5 & 75.9 \\
\hline 4 & 80.0 & 79.9 & 80.2 & 80.2 & 81.8 & 81.7 & 80.5 & 80.2 & 81.9 & 83.4 & 80.2 & 83.2 & 80.2 & 80.3 & 83.3 & 80.5 & 81.4 & 76.2 \\
\hline 5 & 63.7 & 63.7 & 64.1 & 64.0 & 63.8 & 63.5 & 64.3 & 63.3 & 63.7 & 63.3 & 64.1 & 63.6 & 63.4 & 64.1 & 63.5 & 63.8 & 65.5 & 70.4 \\
\hline 6 & 65.6 & 65.7 & 62.0 & 62.1 & 62.1 & 65.7 & 62.0 & 65.7 & 62.1 & 65.8 & 62.2 & 62.1 & 65.7 & 62.3 & 62.1 & 64.0 & 65.6 & 64.1 \\
\hline $1^{\prime}$ & 104.8 & 105.1 & 104.5 & 104.0 & 104.5 & 104.7 & 104.4 & 105.9 & 103.8 & 104.7 & 104.5 & 104.4 & 104.9 & 104.1 & 103.9 & 104.6 & 104.7 & 106.7 \\
\hline $2^{\prime}, 6^{\prime}$ & 167.1 & 167.0 & 167.2 & 167.1 & 167.1 & 167.1 & 167.1 & 166.9 & 167.0 & 167.0 & 167.2 & 167.2 & 167.0 & 167.1 & 167.0 & 167.1 & 164.6 & 164.9 \\
\hline $3^{\prime}, 5^{\prime}$ & 93.6 & 93.9 & 93.1 & 93.5 & 93.1 & 93.5 & 93.1 & 94.2 & 93.4 & 93.4 & 93.1 & 93.0 & 93.9 & 93.5 & 93.4 & 93.5 & 94.0 & 95.9 \\
\hline $4^{\prime}$ & 166.2 & 166.5 & 165.9 & 165.8 & 166.0 & 166.2 & 165.9 & 166.6 & 165.8 & 166.3 & 165.9 & 165.9 & 166.2 & 165.8 & 165.8 & 166.1 & 163.7 & 164.3 \\
\hline $7^{\prime}$ & 207.9 & 207.9 & 207.8 & 207.3 & 207.8 & 207.8 & 207.8 & 208.1 & 207.3 & 207.9 & 207.8 & 207.8 & 208.0 & 207.3 & 207.4 & 207.8 & 203.7 & 204.4 \\
\hline $8^{\prime}$ & 30.6 & 31.0 & 30.8 & 30.9 & 30.7 & 30.7 & 30.7 & 31.0 & 30.9 & 30.6 & 30.7 & 30.9 & 30.4 & 30.9 & 30.9 & 30.8 & 33.8 & 32.9 \\
\hline 1" & 116.6 & 116.7 & 117.9 & 118.1 & 118.1 & 116.7 & 117.9 & 116.0 & 118.3 & 117.0 & 117.4 & 118.1 & 116.0 & 117.6 & 118.3 & 117.3 & 116.8 & 115.6 \\
\hline 2" & 122.8 & 122.7 & 121.9 & 121.9 & 121.5 & 122.3 & 121.7 & 122.9 & 121.5 & 122.3 & 121.9 & 121.5 & 122.9 & 121.9 & 121.6 & 122.3 & 121.6 & 120.0 \\
\hline 3" & 114.0 & 114.1 & 115.3 & 115.3 & 115.4 & 114.1 & 115.3 & 114.0 & 115.4 & 114.2 & 115.3 & 115.5 & 114.0 & 115.4 & 115.5 & 114.6 & 114.3 & 114.3 \\
\hline 4" & 148.0 & 148.0 & 147.8 & 147.9 & 147.9 & 148.0 & 147.9 & 148.0 & 147.9 & 148.1 & 147.9 & 147.9 & 148.0 & 147.9 & 148.0 & 147.9 & 146.2 & 147.5 \\
\hline 5" & 133.3 & 133.4 & 134.1 & 134.1 & 134.2 & 133.4 & 134.1 & 133.2 & 134.3 & 133.5 & 134.2 & 134.3 & 133.1 & 134.3 & 134.4 & 133.7 & 132.6 & 134.9 \\
\hline 6" & 142.1 & 142.2 & 142.1 & 142.2 & 142.2 & 142.1 & 142.1 & 142.2 & 142.3 & 142.2 & 142.1 & 142.2 & 142.1 & 142.2 & 142.3 & 142.2 & 140.7 & 142.6 \\
\hline 7" & 170.1 & 170.1 & 169.9 & 170.0 & 170.4 & 170.3 & 169.9 & 170.3 & 170.4 & 170.1 & 169.9 & 170.3 & 170.2 & 169.9 & 170.4 & 170.1 & 167.5 & 165.6 \\
\hline $1^{\prime \prime \prime}$ & 179.9 & 180.0 & 180.7 & 180.7 & 180.6 & 179.9 & 180.7 & 179.8 & 180.6 & 179.9 & 180.6 & 180.6 & 179.8 & 180.6 & 180.5 & 180.3 & 177.3 & 175.6 \\
\hline $2^{\prime \prime \prime}$ & 46.2 & 46.1 & 49.4 & 49.4 & 49.3 & 46.1 & 49.5 & 46.8 & 49.3 & 46.0 & 49.9 & 49.4 & 46.8 & 49.9 & 49.3 & 47.7 & 50.0 & 46.9 \\
\hline 3"' & 42.6 & 42.6 & 44.8 & 44.8 & 44.8 & 42.6 & 44.8 & 44.1 & 44.8 & 42.5 & 45.2 & 44.9 & 44.1 & 45.3 & 44.9 & 43.7 & 46.2 & 43.6 \\
\hline 4"' & 28.0 & 28.0 & 27.6 & 27.6 & 27.6 & 28.0 & 27.6 & 29.2 & 27.6 & 28.0 & 29.4 & 27.6 & 29.1 & 29.4 & 27.6 & 27.9 & 31.0 & 30.4 \\
\hline $5^{\prime \prime \prime}$ & 177.0 & 177.0 & 176.9 & 176.9 & 176.8 & 176.9 & 176.8 & 174.7 & 176.8 & 177.0 & 174.7 & 176.8 & 174.6 & 174.8 & 176.8 & 176.7 & 173.9 & 172.2 \\
\hline $6^{\prime \prime \prime}$ & 178.0 & 178.1 & 178.4 & 178.4 & 178.2 & 177.9 & 178.5 & 178.1 & 178.2 & 177.9 & 178.3 & 178.3 & 178.0 & 178.2 & 178.3 & 178.2 & 175.3 & 172.1 \\
\hline
\end{tabular}


Figure S9. Correlation plots of experimental ${ }^{1} \mathrm{H}$ NMR chemical shifts versus corresponding calculated ${ }^{1} \mathrm{H}$ NMR chemical shifts of $\mathbf{3}$.
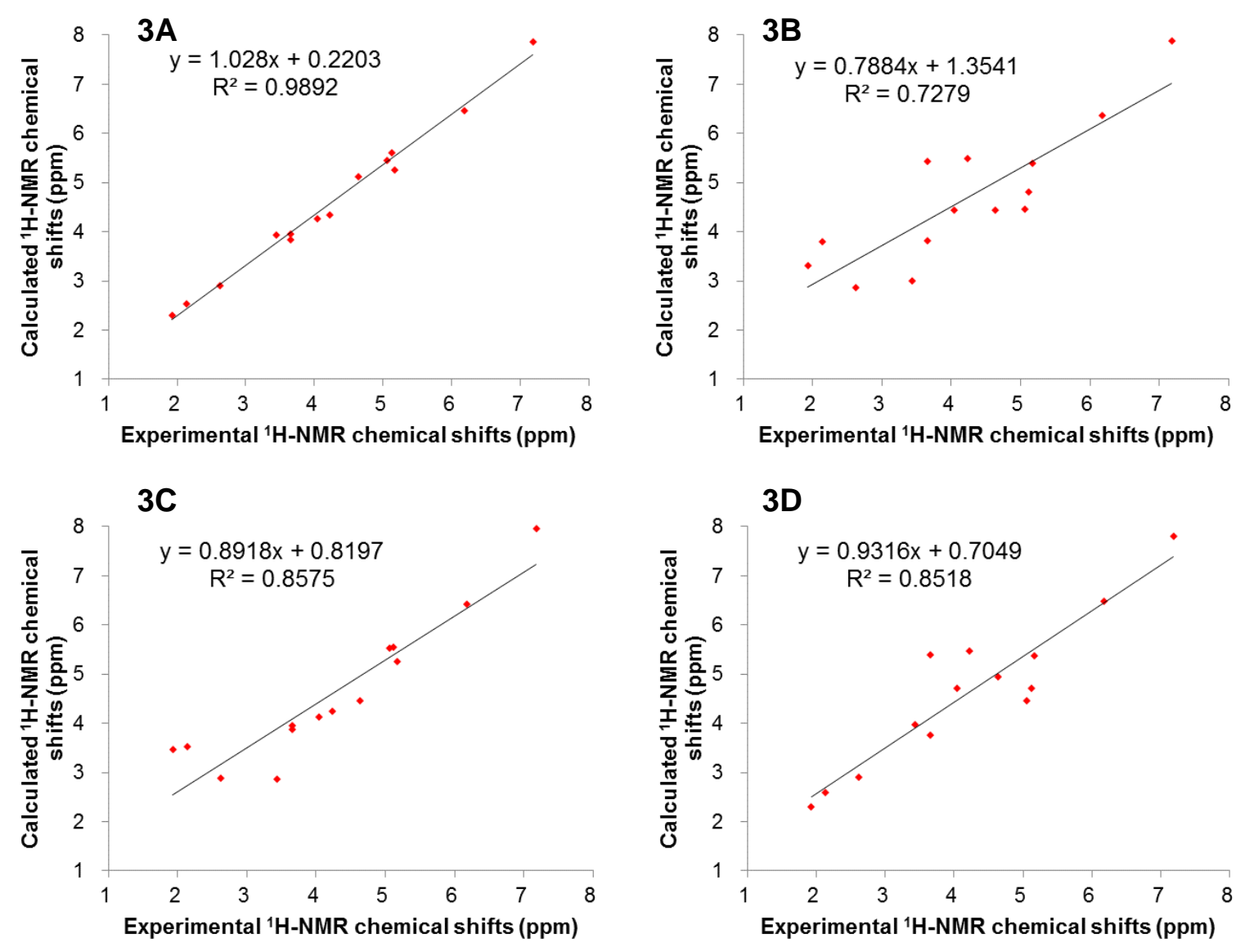
Figure S10. Correlation plots of experimental ${ }^{13} \mathrm{C}$ NMR chemical shifts versus corresponding calculated ${ }^{13} \mathrm{C}$ NMR chemical shifts of 3 .
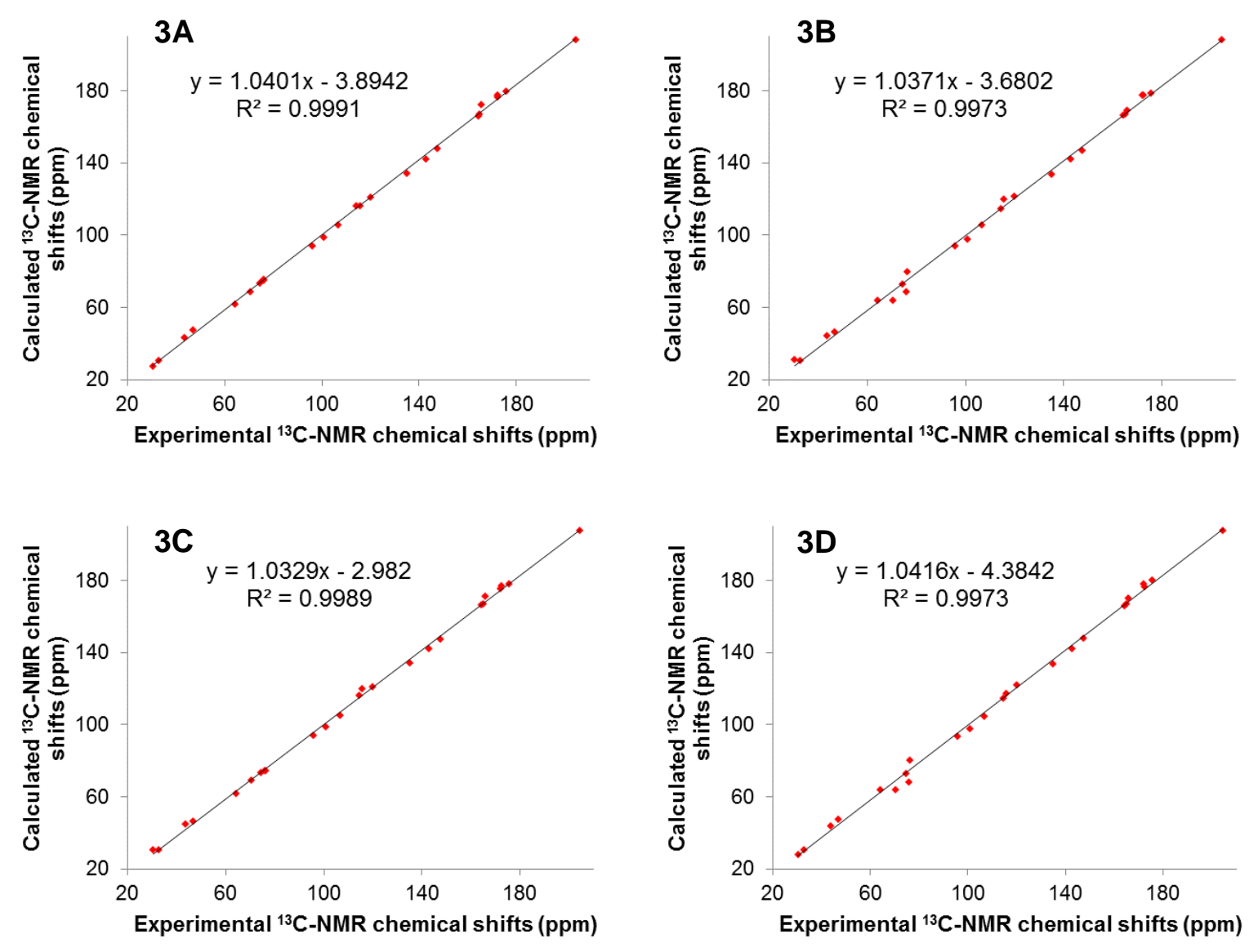
Table S81. Statistical parameters of calculated ${ }^{1} \mathrm{H}$ NMR chemical shifts of $\mathbf{3}(\mathrm{ppm})$.

\begin{tabular}{cccc}
\hline & $R^{2}$ & CMaxErr & CMAE \\
\hline 3A & $\mathbf{0 . 9 8 9 2}$ & $\mathbf{0 . 2 8}$ & $\mathbf{0 . 1 3}$ \\
3B & 0.7279 & 1.52 & 0.76 \\
3C & 0.8575 & 1.15 & 0.47 \\
3D & 0.8518 & 1.38 & 0.43 \\
\hline
\end{tabular}

$R^{2}$ : coefficient of determination; CMaxErr: corrected maximum absolute error with respect to the linear fit; CMAE: corrected mean absolute error with respect to the linear fit

Table S82. Statistical parameters of calculated ${ }^{13} \mathrm{C}$ NMR chemical shifts of $\mathbf{3}(\mathrm{ppm})$.

\begin{tabular}{cccc}
\hline & $R^{2}$ & CMaxErr & CMAE \\
\hline 3A & $\mathbf{0 . 9 9 9 1}$ & 3.8 & $\mathbf{1 . 2}$ \\
3B & 0.9973 & 6.1 & 2.0 \\
3C & 0.9989 & $\mathbf{3 . 4}$ & 1.3 \\
3D & 0.9973 & 6.4 & 2.0 \\
\hline
\end{tabular}

$R^{2}$ : coefficient of determination; CMaxErr: corrected maximum absolute error with respect to the linear fit; CMAE:

corrected mean absolute error with respect to the linear fit 
Table S83. Calculated ${ }^{1} \mathrm{H}$ NMR coupling constants between H-2"' and H-3"' in 3A (ppm).

\begin{tabular}{|c|c|c|c|c|c|c|c|c|c|c|c|c|c|c|}
\hline & \multicolumn{13}{|c|}{ calculated $^{a}$} & \multirow{2}{*}{$\frac{\text { experimental }^{b}}{3}$} \\
\hline & $3 \mathrm{~A}-1$ & 3A-2 & $3 \mathrm{~A}-3$ & $3 A-4$ & $3 A-5$ & $3 A-6$ & $3 \mathrm{~A}-7$ & 3A-8 & 3A-9 & 3A-10 & 3A-11 & $\begin{array}{c}\text { averaged- } \\
\mathbf{3 A}^{c}\end{array}$ & $\begin{array}{c}\text { averaged-3A } \\
\text { (corrected) }^{d}\end{array}$ & \\
\hline$J_{2^{\prime \prime-3} 3^{\prime \prime \prime}}$ & 3.3 & 3.3 & 3.5 & 3.5 & 3.4 & 3.4 & 3.0 & 3.0 & 3.2 & 3.3 & 3.2 & 3.3 & 3.1 & 3.2 \\
\hline
\end{tabular}

${ }^{a}$ Calculated at the B3LYP/6-31G(d,p)u+1s (using only the Fermi contact term) level in acetone (PCM). ${ }^{b}$ Measured in acetone- $d_{6}(500 \mathrm{MHz}) .{ }^{c}$ Averaged according to

the Boltzmann distribution theory at $298 \mathrm{~K}$ based on relative Gibbs free energies. ${ }^{d}$ Scaled by using the slope parameter 0.94.

Table S84. Calculated ${ }^{1} \mathrm{H}$ NMR coupling constants between $\mathrm{H}-2^{\prime \prime \prime}$ and $\mathrm{H}-3^{\prime \prime \prime}$ in $\mathbf{3 B}$ (ppm).

\begin{tabular}{|c|c|c|c|c|c|c|c|c|c|c|c|c|c|c|c|c|c|c|}
\hline & & & & & & & & & calcul & & & & & & & & & experimental $^{b}$ \\
\hline & 3B-1 & $3 B-2$ & $3 B-3$ & $3 B-4$ & $3 B-5$ & 3B-6 & 3B-7 & 3B-8 & 3B-9 & 3B-10 & 3B-11 & 3B-12 & 3B-13 & 3B-14 & 3B-15 & $\begin{array}{c}\text { averaged- } \\
\mathbf{3 B}^{c}\end{array}$ & $\begin{array}{c}\text { averaged-3B } \\
\text { (corrected) }^{d}\end{array}$ & 3 \\
\hline$J_{2^{\prime \prime}-3^{\prime \prime \prime}}$ & 12.9 & 12.9 & 7.0 & 12.9 & 7.2 & 12.7 & 12.9 & 8.4 & 12.1 & 8.3 & 12.1 & 7.2 & 8.4 & 12.7 & 8.4 & 11.1 & 10.4 & 3.2 \\
\hline
\end{tabular}

${ }^{a}$ Calculated at the B3LYP/6-31G(d,p)u+1s (using only the Fermi contact term) level in acetone (PCM). ${ }^{b}$ Measured in acetone- ${ }_{6}\left(500\right.$ MHz). ${ }^{c}$ Averaged according to the Boltzmann distribution theory at $298 \mathrm{~K}$ based on relative Gibbs free energies. ${ }^{d}$ Scaled by using the slope parameter 0.94 .

Table S85. Calculated ${ }^{1} \mathrm{H}$ NMR coupling constants between $\mathrm{H}-2^{\prime \prime \prime}$ and $\mathrm{H}-3^{\prime \prime \prime}$ in $\mathbf{3 C}$ (ppm).

\begin{tabular}{|c|c|c|c|c|c|c|c|c|c|c|c|c|c|}
\hline & & & & & & & culated $^{a}$ & & & & & & experimental $^{b}$ \\
\hline & 3C-1 & $3 \mathrm{C}-2$ & $3 C-3$ & $3 C-4$ & $3 C-5$ & $3 C-6$ & $3 C-7$ & $3 C-8$ & 3C-9 & $3 C-10$ & $\begin{array}{c}\text { averaged- } \\
\mathbf{3 C}^{\mathrm{c}} \\
\end{array}$ & $\begin{array}{l}\text { averaged-3C } \\
\text { (corrected) }^{d}\end{array}$ & 3 \\
\hline$J_{2^{\prime \prime \prime}-3 " '}$ & 12.2 & 12.5 & 12.4 & 12.5 & 11.4 & 12.2 & 12.4 & 12.7 & 12.3 & 12.3 & 12.3 & 11.6 & 3.2 \\
\hline
\end{tabular}


Table S86. Calculated ${ }^{1} \mathrm{H}$ NMR coupling constants between H-2"' and H-3"' in 3D (ppm).

\begin{tabular}{|c|c|c|c|c|c|c|c|c|c|c|c|c|c|c|c|c|c|c|}
\hline & \multicolumn{17}{|c|}{ calculated $^{a}$} & \multirow{2}{*}{$\begin{array}{c}\text { experimental }^{b} \\
3\end{array}$} \\
\hline & 3D-1 & $3 D-2$ & 3D-3 & 3D-4 & $3 D-5$ & $3 D-6$ & 3D-7 & 3D-8 & 3D-9 & 3D-10 & 3D-11 & 3D-12 & 3D-13 & 3D-14 & 3D-15 & $\begin{array}{c}\text { averaged- } \\
\text { 3D }^{c}\end{array}$ & $\begin{array}{l}\text { averaged-3D } \\
\text { (corrected) }^{d}\end{array}$ & \\
\hline$J_{2^{\prime \prime-1}-3^{\prime \prime \prime}}$ & 4.3 & 4.3 & 1.8 & 1.8 & 1.8 & 4.4 & 1.8 & 3.9 & 1.8 & 4.4 & 1.7 & 1.8 & 3.9 & 1.7 & 1.8 & 3.1 & 2.9 & 3.2 \\
\hline
\end{tabular}

Calculated at the B3LYP/6-31G(d,p)u+1s (using only the Fermi contact term) level in acetone (PCM). ${ }^{b}$ Measured in acetone- $d_{6}(500 \mathrm{MHz}) .{ }^{c}$ Averaged according to the Boltzmann distribution theory at $298 \mathrm{~K}$ based on relative Gibbs free energies. ${ }^{d}$ Scaled by using the slope parameter 0.94 . 
Table S87. Important thermodynamic parameters and conformational analysis of $\mathbf{3 A}$ at the B3LYP/6-31G(d,p) level in MeOH (PCM).

\begin{tabular}{cllllll}
\hline conformers & $E$ (a.u.) & $E^{\prime}$ (a.u.) & $H$ (a.u.) & $G$ (a.u.) & $\Delta G(\mathrm{kcal} / \mathrm{mol})$ & $\mathrm{P}_{\mathrm{G}}(\%)$ \\
\hline 3A-1 & -2322.236521 & -2321.730439 & -2321.690407 & -2321.803695 & 0.00 & 25.0 \\
3A-2 & -2322.235877 & -2321.729843 & -2321.689788 & -2321.803148 & 0.34 & 14.0 \\
3A-3 & -2322.234567 & -2321.729179 & -2321.688777 & -2321.803126 & 0.36 & 13.7 \\
3A-4 & -2322.234060 & -2321.728826 & -2321.688378 & -2321.802986 & 0.44 & 11.8 \\
3A-5 & -2322.234362 & -2321.728814 & -2321.688521 & -2321.802727 & 0.61 & 9.0 \\
3A-6 & -2322.234903 & -2321.729164 & -2321.688939 & -2321.802715 & 0.61 & 8.9 \\
3A-7 & -2322.234287 & -2321.728387 & -2321.688275 & -2321.802284 & 0.89 & 5.6 \\
3A-8 & -2322.233602 & -2321.727751 & -2321.687601 & -2321.801835 & 1.17 & 3.5 \\
3A-9 & -2322.232641 & -2321.726979 & -2321.686708 & -2321.801016 & 1.68 & 1.5 \\
3A-10 & -2322.232060 & -2321.726573 & -2321.686235 & -2321.800976 & 1.71 & 1.4 \\
3A-11 & -2322.232314 & -2321.726960 & -2321.686530 & -2321.800971 & 1.71 & 1.4 \\
3A-12 & -2322.231763 & -2321.726517 & -2321.686069 & -2321.800674 & 1.90 & 1.0 \\
\hline
\end{tabular}

E: total energy; $E$ ': total energy with zero point energy; H: enthalpy; G: Gibbs free energy; $\triangle G$ : relative Gibbs free energy at the B3LYP/6-31G(d,p) level in MeOH (PCM). $P_{G}$ : conformational distribution calculated from relative Gibbs free energy.

Table S88. Important thermodynamic parameters and conformational analysis of $\mathbf{3 B}$ at the B3LYP/6-31G(d,p) level in MeOH (PCM).

\begin{tabular}{cllllll}
\hline conformers & $E$ (a.u.) & $E^{\prime}$ (a.u.) & $H$ (a.u.) & $G$ (a.u.) & $\Delta G(\mathrm{kcal} / \mathrm{mol})$ & $\mathrm{P}_{\mathrm{G}}(\%)$ \\
\hline 3B-1 & -2322.226742 & -2321.721094 & -2321.680783 & -2321.794803 & 0.00 & 23.0 \\
3B-2 & -2322.226431 & -2321.720686 & -2321.680382 & -2321.794284 & 0.33 & 13.3 \\
3B-3 & -2322.226512 & -2321.720703 & -2321.680473 & -2321.794147 & 0.41 & 11.5 \\
3B-4 & -2322.225576 & -2321.720004 & -2321.679660 & -2321.793980 & 0.52 & 9.6 \\
3B-5 & -2322.225801 & -2321.719993 & -2321.679773 & -2321.793345 & 0.91 & 4.9 \\
3B-6 & -2322.224189 & -2321.718631 & -2321.678234 & -2321.793257 & 0.97 & 4.5 \\
3B-7 & -2322.225143 & -2321.719478 & -2321.679150 & -2321.793154 & 1.03 & 4.0 \\
3B-8 & -2322.225419 & -2321.719591 & -2321.679334 & -2321.793021 & 1.12 & 3.5 \\
3B-9 & -2322.224989 & -2321.719175 & -2321.678884 & -2321.792829 & 1.24 & 2.8 \\
3B-10 & -2322.224951 & -2321.719307 & -2321.678955 & -2321.792823 & 1.24 & 2.8 \\
3B-11 & -2322.224602 & -2321.718934 & -2321.678577 & -2321.792640 & 1.36 & 2.3 \\
3B-12 & -2322.225212 & -2321.719339 & -2321.679131 & -2321.792585 & 1.39 & 2.2 \\
3B-13 & -2322.224349 & -2321.718724 & -2321.678372 & -2321.792415 & 1.50 & 1.8 \\
3B-14 & -2322.223814 & -2321.718122 & -2321.677762 & -2321.792370 & 1.53 & 1.7 \\
3B-15 & -2322.223785 & -2321.718158 & -2321.677773 & -2321.792003 & 1.76 & 1.2 \\
\hline
\end{tabular}

E: total energy; $E$ ': total energy with zero point energy; H: enthalpy; G: Gibbs free energy; $\triangle$ G: relative Gibbs free energy at the B3LYP/6-31G(d,p) level in MeOH $(P C M) . P_{G}$ : conformational distribution calculated from relative Gibbs free energy. 
Table S89. Important thermodynamic parameters and conformational analysis of $\mathbf{3 C}$ at the B3LYP/6-31G(d,p) level in MeOH (PCM).

\begin{tabular}{cllllll}
\hline conformers & $E$ (a.u.) & $E^{\prime}$ (a.u.) & $H$ (a.u.) & $G$ (a.u.) & $\Delta G(\mathrm{kcal} / \mathrm{mol})$ & $\mathrm{P}_{\mathrm{G}}(\%)$ \\
\hline $\mathbf{3 C - 1}$ & -2322.232569 & -2321.726477 & -2321.686482 & -2321.799672 & 0.00 & 30.2 \\
$\mathbf{3 C - 2}$ & -2322.231226 & -2321.725602 & -2321.685356 & -2321.799231 & 0.28 & 19.0 \\
$\mathbf{3 C - 3}$ & -2322.231236 & -2321.725454 & -2321.685293 & -2321.799059 & 0.38 & 15.8 \\
$\mathbf{3 C}-\mathbf{4}$ & -2322.230659 & -2321.725082 & -2321.684809 & -2321.798703 & 0.61 & 10.8 \\
$\mathbf{3 C - 5}$ & -2322.230265 & -2321.724776 & -2321.684475 & -2321.798250 & 0.89 & 6.7 \\
$\mathbf{3 C}-\mathbf{6}$ & -2322.229937 & -2321.723930 & -2321.683873 & -2321.797509 & 1.36 & 3.1 \\
$\mathbf{3 C - 7}$ & -2322.228627 & -2321.723021 & -2321.682732 & -2321.797044 & 1.65 & 1.9 \\
$\mathbf{3 C - 8}$ & -2322.228615 & -2321.722870 & -2321.682676 & -2321.796623 & 1.91 & 1.2 \\
$\mathbf{3 C - 9}$ & -2322.229172 & -2321.723221 & -2321.683198 & -2321.796517 & 1.98 & 1.1 \\
\hline
\end{tabular}

E: total energy; $E^{\prime}$ : total energy with zero point energy; H: enthalpy; G: Gibbs free energy; $\triangle$ G: relative Gibbs free energy at the B3LYP/6-3IG(d,p) level in $\mathrm{MeOH}(\mathrm{PCM}) . \mathrm{P}_{G}$ : conformational distribution calculated from relative Gibbs free energy.

Table S90. Important thermodynamic parameters and conformational analysis of $\mathbf{3 D}$ at the B3LYP/6-31G(d,p) level in MeOH (PCM).

\begin{tabular}{cllllll}
\hline conformers & $E$ (a.u.) & $E^{\prime}($ a.u. $)$ & $H$ (a.u. $)$ & $G$ (a.u. $)$ & $\Delta G(\mathrm{kcal} / \mathrm{mol})$ & $\mathrm{P}_{\mathrm{G}}(\%)$ \\
\hline 3D-1 & -2322.228679 & -2321.723305 & -2321.682794 & -2321.797814 & 0.00 & 21.0 \\
3D-2 & -2322.228304 & -2321.723035 & -2321.682483 & -2321.797753 & 0.04 & 19.7 \\
3D-3 & -2322.229750 & -2321.724012 & -2321.683712 & -2321.797564 & 0.16 & 16.1 \\
3D-4 & -2322.229253 & -2321.723595 & -2321.683255 & -2321.797332 & 0.30 & 12.6 \\
3D-5 & -2322.228583 & -2321.722803 & -2321.682533 & -2321.796252 & 0.98 & 4.0 \\
3D-6 & -2322.227350 & -2321.721897 & -2321.681432 & -2321.796190 & 1.02 & 3.8 \\
3D-7 & -2322.228491 & -2321.722434 & -2321.682315 & -2321.795852 & 1.23 & 2.6 \\
3D-8 & -2322.226116 & -2321.720857 & -2321.680278 & -2321.795598 & 1.24 & 2.6 \\
3D-9 & -2322.227949 & -2321.722238 & -2321.681925 & -2321.795831 & 1.28 & 2.4 \\
3D-10 & -2322.226471 & -2321.721261 & -2321.680676 & -2321.795776 & 1.39 & 2.0 \\
3D-11 & -2322.227651 & -2321.721877 & -2321.681573 & -2321.795572 & 1.39 & 2.0 \\
3D-12 & -2322.227715 & -2321.722084 & -2321.681747 & -2321.795605 & 1.41 & 2.0 \\
3D-13 & -2322.226500 & -2321.721117 & -2321.680606 & -2321.795453 & 1.48 & 1.7 \\
3D-14 & -2322.227202 & -2321.721455 & -2321.681138 & -2321.795204 & 1.64 & 1.3 \\
3D-15 & -2322.227196 & -2321.721564 & -2321.681202 & -2321.795128 & 1.69 & 1.2 \\
\hline
\end{tabular}

E: total energy; $E$ ': total energy with zero point energy; H: enthalpy; G: Gibbs free energy; $\triangle$ G: relative Gibbs free energy at the B3LYP/6-31G(d,p) level in MeOH (PCM). $P_{G}$ : conformational distribution calculated from relative Gibbs free energy. 
Figure S11. Possible structures of balanophotannin F (4).

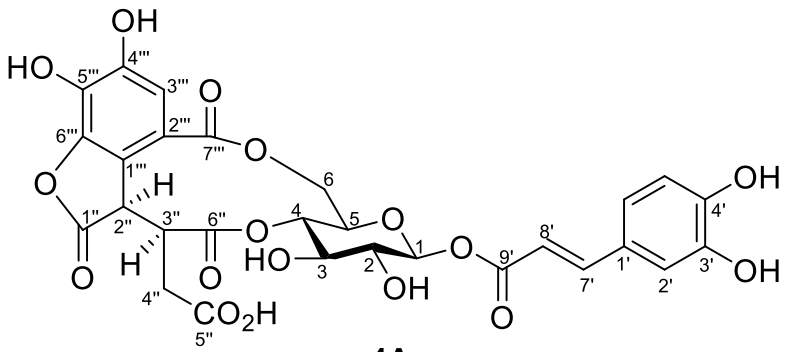

$4 \mathrm{~A}$

(2"R,3"S)

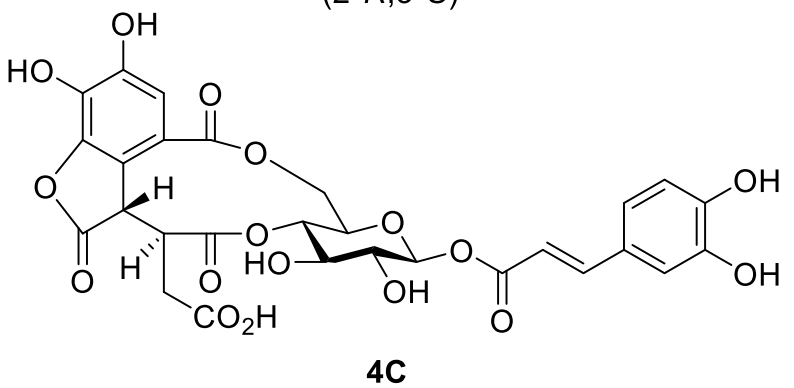

(2"S,3"S)

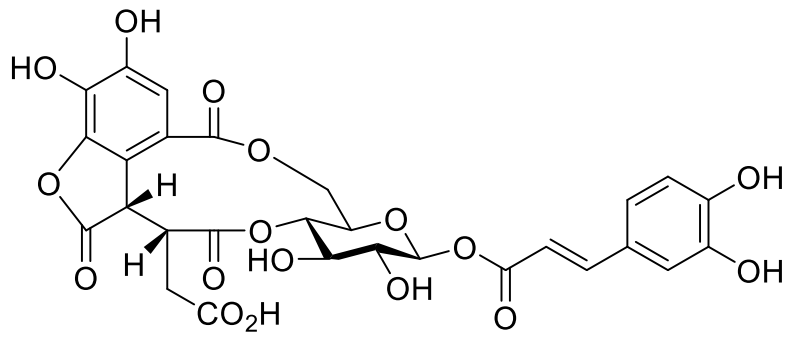

4B

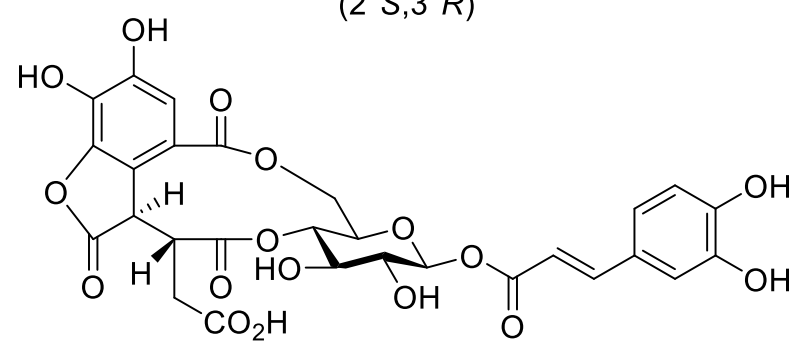

4D

(2"R,3"R) 
Figure S12. Lowest-energy conformers of $\mathbf{4}$ and Boltzmann-weighted calculated $J_{2^{\prime \prime}-3^{\prime \prime}}$ values (B3LYP/6-31G(d,p)u+1s level in MeOH (PCM)).

$J_{\text {exptl }}\left(J_{\text {calcd }}\right)$ in $\mathrm{Hz}$

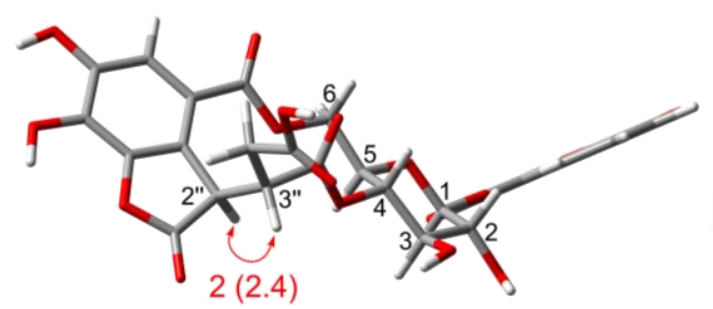

4A-1

$(2 " R, 3 " S)$

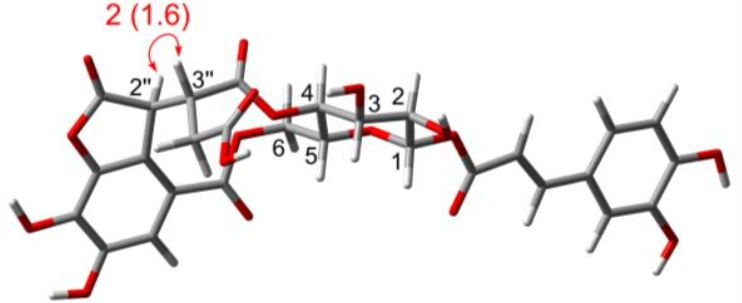

4B-1

$(2 " S, 3 " R)$

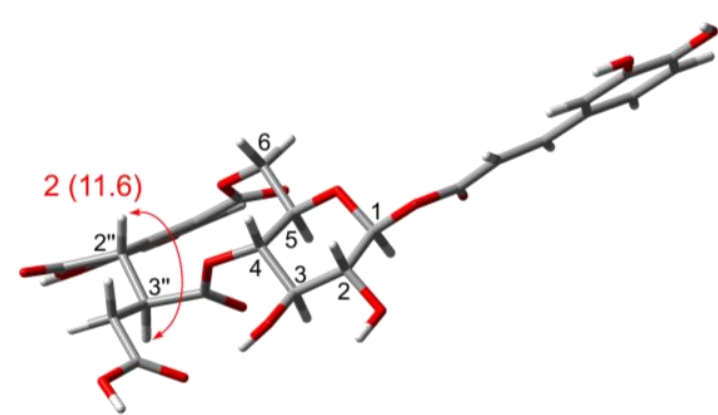

4C-1

(2"S,3"S)

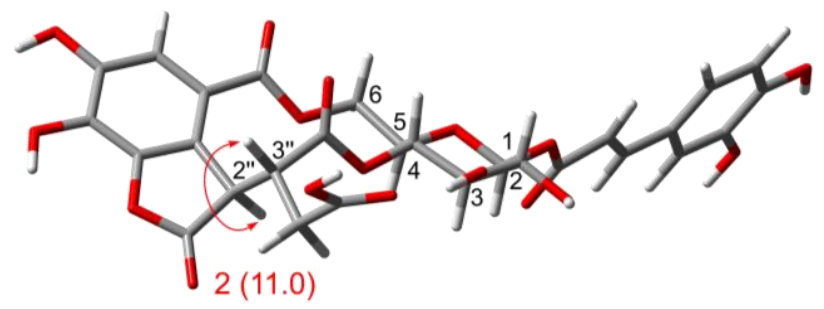

4D-1

(2"R,3"R) 
Table S91. Important thermodynamic parameters and conformational analysis of $\mathbf{4 A}$ at the B3LYP/6-31G(d,p) level in MeOH (PCM).

\begin{tabular}{clllllr}
\hline conformers & $E$ (a.u.) & $E^{\prime}$ (a.u.) & $H$ (a.u.) & $G$ (a.u.) & $\Delta G(\mathrm{kcal} / \mathrm{mol})$ & $\mathrm{P}_{\mathrm{G}}(\%)$ \\
\hline $\mathbf{4 A - 1}$ & -2360.335941 & -2359.825015 & -2359.783179 & -2359.903419 & 0.00 & 27.1 \\
$\mathbf{4 A - 2}$ & -2360.336498 & -2359.825356 & -2359.783622 & -2359.902893 & 0.33 & 15.5 \\
$\mathbf{4 A - 3}$ & -2360.336126 & -2359.825009 & -2359.783287 & -2359.902631 & 0.49 & 11.8 \\
$\mathbf{4 A - 4}$ & -2360.335948 & -2359.824862 & -2359.783107 & -2359.902409 & 0.63 & 9.3 \\
$\mathbf{4 A - 5}$ & -2360.334856 & -2359.823740 & -2359.781999 & -2359.902237 & 0.74 & 7.7 \\
$\mathbf{4 A - 6}$ & -2360.335178 & -2359.824081 & -2359.782337 & -2359.902087 & 0.84 & 6.6 \\
$\mathbf{4 A - 7}$ & -2360.335583 & -2359.824474 & -2359.782739 & -2359.902042 & 0.86 & 6.3 \\
$\mathbf{4 A - 8}$ & -2360.334180 & -2359.823013 & -2359.781437 & -2359.901150 & 1.42 & 2.4 \\
$\mathbf{4 A - 9}$ & -2360.334918 & -2359.823724 & -2359.782119 & -2359.900817 & 1.63 & 1.7 \\
$\mathbf{4 A - 1 0}$ & -2360.334657 & -2359.823463 & -2359.781736 & -2359.900763 & 1.67 & 1.6 \\
$\mathbf{4 A - 1 1}$ & -2360.335113 & -2359.823690 & -2359.782205 & -2359.900636 & 1.75 & 1.4 \\
$\mathbf{4 A - 1 2}$ & -2360.333201 & -2359.821995 & -2359.780286 & -2359.900485 & 1.84 & 1.2 \\
$\mathbf{4 A - 1 3}$ & -2360.334506 & -2359.823279 & -2359.781586 & -2359.900405 & 1.89 & 1.1 \\
$\mathbf{4 A - 1 4}$ & -2360.334128 & -2359.822949 & -2359.781253 & -2359.900367 & 1.92 & 1.1 \\
\hline
\end{tabular}

E: total energy; $E$ ': total energy with zero point energy; H: enthalpy; G: Gibbs free energy; $\triangle$ G: relative Gibbs free energy at the B3LYP/6-31G(d,p) level in MeOH (PCM). $P_{G}$ : conformational distribution calculated from relative Gibbs free energy.

Table S92. Important thermodynamic parameters and conformational analysis of $\mathbf{4 B}$ at the B3LYP/6-31G(d,p) level in MeOH (PCM).

\begin{tabular}{clllllc}
\hline conformers & $E$ (a.u.) & $E^{\prime}($ a.u. $)$ & $H$ (a.u.) & $G$ (a.u.) & $\Delta G(\mathrm{kcal} / \mathrm{mol})$ & $\mathrm{P}_{\mathrm{G}}(\%)$ \\
\hline 4B -1 & -2360.335336 & -2359.823454 & -2359.782133 & -2359.899729 & 0.00 & 27.3 \\
4B -2 & -2360.335512 & -2359.823511 & -2359.782290 & -2359.899454 & 0.17 & 20.4 \\
$\mathbf{4 B - 3}$ & -2360.334128 & -2359.822288 & -2359.780881 & -2359.899330 & 0.25 & 17.9 \\
$\mathbf{4 B - 4}$ & -2360.334664 & -2359.822699 & -2359.781389 & -2359.899165 & 0.35 & 15.0 \\
$\mathbf{4 B - 5}$ & -2360.333740 & -2359.821890 & -2359.780531 & -2359.898507 & 0.77 & 7.5 \\
4B-6 & -2360.331317 & -2359.819967 & -2359.778337 & -2359.897077 & 1.66 & 1.6 \\
4B -7 & -2360.331822 & -2359.820150 & -2359.778677 & -2359.896755 & 1.87 & 1.2 \\
\hline
\end{tabular}

E: total energy; $E^{\prime}$ : total energy with zero point energy; H: enthalpy; G: Gibbs free energy; $\triangle$ G: relative Gibbs free energy at the B3LYP/6-31G(d,p) level in MeOH $(P C M) . P_{G}$ : conformational distribution calculated from relative Gibbs free energy. 
Table S93. Important thermodynamic parameters and conformational analysis of $\mathbf{4 C}$ at the B3LYP/6-31G(d,p) level in MeOH (PCM).

\begin{tabular}{cllllll}
\hline conformers & $E$ (a.u.) & $E^{\prime}$ (a.u.) & $H$ (a.u.) & $G$ (a.u.) & $\Delta G(\mathrm{kcal} / \mathrm{mol})$ & $\mathrm{P}_{\mathrm{G}}(\%)$ \\
\hline $\mathbf{4 C - 1}$ & -2360.332687 & -2359.820355 & -2359.779315 & -2359.895742 & 0.00 & 22.7 \\
$\mathbf{4 C - 2}$ & -2360.332373 & -2359.820071 & -2359.779026 & -2359.895557 & 0.12 & 18.7 \\
$\mathbf{4 C - 3}$ & -2360.332188 & -2359.819939 & -2359.778847 & -2359.895300 & 0.28 & 14.2 \\
$\mathbf{4 C - 4}$ & -2360.331768 & -2359.819579 & -2359.778486 & -2359.895239 & 0.32 & 13.3 \\
$\mathbf{4 C - 5}$ & -2360.331096 & -2359.818751 & -2359.777672 & -2359.894954 & 0.49 & 9.9 \\
$\mathbf{4 C - 6}$ & -2360.331490 & -2359.818930 & -2359.777947 & -2359.894372 & 0.86 & 5.3 \\
$\mathbf{4 C - 7}$ & -2360.330905 & -2359.818520 & -2359.777453 & -2359.894170 & 0.99 & 4.3 \\
$\mathbf{4 C - 8}$ & -2360.330559 & -2359.818221 & -2359.777163 & -2359.893757 & 1.25 & 2.8 \\
$\mathbf{4 C - 9}$ & -2360.329350 & -2359.817439 & -2359.776232 & -2359.893043 & 1.69 & 1.3 \\
\hline
\end{tabular}

E: total energy; $E$ ': total energy with zero point energy; H: enthalpy; G: Gibbs free energy; $\Delta G$ : relative Gibbs free energy at the B3LYP/6-31G(d,p) level in MeOH (PCM). $P_{G}$ : conformational distribution calculated from relative Gibbs free energy.

Table S94. Important thermodynamic parameters and conformational analysis of 4D at the B3LYP/6-31G(d,p) level in MeOH (PCM).

\begin{tabular}{|c|c|c|c|c|c|c|}
\hline conformers & $E$ (a.u.) & $E^{\prime}$ (a.u.) & $H$ (a.u.) & $G$ (a.u.) & $\Delta G(\mathrm{kcal} / \mathrm{mol})$ & $\mathrm{P}_{\mathrm{G}}(\%)$ \\
\hline 4D-1 & -2360.334165 & -2359.822107 & -2359.780775 & -2359.899057 & 0.00 & 18.3 \\
\hline 4D-2 & -2360.334706 & -2359.822466 & -2359.781216 & -2359.898672 & 0.24 & 12.2 \\
\hline 4D-3 & -2360.336956 & -2359.824089 & -2359.783441 & -2359.898657 & 0.25 & 12.0 \\
\hline 4D-4 & -2360.334351 & -2359.822077 & -2359.780858 & -2359.898188 & 0.55 & 7.3 \\
\hline 4D-5 & -2360.333805 & -2359.821623 & -2359.780360 & -2359.898126 & 0.58 & 6.8 \\
\hline 4D-6 & -2360.335657 & -2359.822864 & -2359.782152 & -2359.898071 & 0.62 & 6.4 \\
\hline 4D-7 & -2360.336425 & -2359.823506 & -2359.782839 & -2359.897983 & 0.67 & 5.9 \\
\hline 4D-8 & -2360.333498 & -2359.821311 & -2359.780033 & -2359.897871 & 0.74 & 5.2 \\
\hline 4D-9 & -2360.332971 & -2359.820935 & -2359.779586 & -2359.897671 & 0.87 & 4.2 \\
\hline 4D-10 & -2360.335154 & -2359.822395 & -2359.781647 & -2359.897582 & 0.93 & 3.8 \\
\hline 4D-11 & -2360.336622 & -2359.823550 & -2359.783024 & -2359.897449 & 1.01 & 3.3 \\
\hline 4D-12 & -2360.336057 & -2359.823052 & -2359.782460 & -2359.897215 & 1.16 & 2.6 \\
\hline 4D-13 & -2360.333174 & -2359.820889 & -2359.779674 & -2359.897039 & 1.27 & 2.2 \\
\hline 4D-14 & -2360.332604 & -2359.820477 & -2359.779196 & -2359.896886 & 1.36 & 1.8 \\
\hline 4D-15 & -2360.334792 & -2359.821912 & -2359.781236 & -2359.896774 & 1.43 & 1.6 \\
\hline
\end{tabular}

E: total energy; E': total energy with zero point energy; H: enthalpy; G: Gibbs free energy; $\triangle$ G: relative Gibbs free energy at the B3LYP/6-3IG(d,p) level in MeOH $(P C M) . P_{G}$ : conformational distribution calculated from relative Gibbs free energy. 
Table S95. Calculated ${ }^{1} \mathrm{H}$ NMR chemical shifts of $\mathbf{4 A}$.

\begin{tabular}{|c|c|c|c|c|c|c|c|c|c|c|c|c|c|c|c|c|c|}
\hline \multirow[b]{2}{*}{ Position } & \multicolumn{16}{|c|}{ calculated $^{a}$} & \multirow{2}{*}{$\begin{array}{c}\text { experimental }^{b} \\
4\end{array}$} \\
\hline & 4A-1 & $4 A-2$ & $4 A-3$ & $4 A-4$ & $4 A-5$ & $4 A-6$ & 4A-7 & $4 \mathrm{~A}-8$ & 4A-9 & $4 \mathrm{~A}-10$ & $4 \mathrm{~A}-11$ & $4 \mathrm{~A}-12$ & 4A-13 & 4A-14 & $\begin{array}{c}\text { averaged- } \\
\mathbf{4 A}^{c}\end{array}$ & $\begin{array}{c}\text { averaged-4A }^{\text {(corrected) }}{ }^{d}\end{array}$ & \\
\hline 1 & 6.17 & 6.20 & 6.22 & 6.18 & 6.15 & 6.15 & 6.19 & 6.20 & 6.21 & 6.11 & 6.24 & 6.15 & 6.19 & 6.21 & 6.18 & 5.61 & 5.70 \\
\hline 2 & 4.03 & 4.06 & 4.07 & 4.02 & 4.13 & 4.13 & 4.02 & 3.78 & 3.74 & 4.12 & 3.76 & 4.12 & 4.07 & 4.08 & 4.04 & 3.57 & 3.64 \\
\hline 3 & 4.22 & 4.25 & 4.25 & 4.22 & 4.27 & 4.26 & 4.22 & 4.31 & 4.23 & 4.25 & 4.25 & 4.23 & 4.23 & 4.24 & 4.24 & 3.76 & 3.90 \\
\hline 4 & 5.39 & 5.39 & 5.39 & 5.39 & 5.39 & 5.39 & 5.39 & 5.47 & 5.41 & 5.41 & 5.41 & 5.42 & 5.43 & 5.44 & 5.39 & 4.86 & 4.93 \\
\hline 5 & 4.26 & 4.27 & 4.27 & 4.26 & 4.25 & 4.24 & 4.26 & 4.32 & 4.25 & 4.23 & 4.27 & 4.20 & 4.24 & 4.25 & 4.26 & 3.78 & 4.05 \\
\hline \multirow[t]{2}{*}{6} & 4.51 & 4.50 & 4.51 & 4.52 & 4.54 & 4.54 & 4.52 & 4.63 & 4.56 & 4.54 & 4.59 & 4.48 & 4.47 & 4.48 & 4.52 & 4.02 & 4.16 \\
\hline & 4.28 & 4.31 & 4.31 & 4.30 & 4.32 & 4.32 & 4.29 & 4.34 & 4.35 & 4.34 & 4.34 & 4.38 & 4.36 & 4.35 & 4.31 & 3.82 & 4.08 \\
\hline $2^{\prime}$ & 7.41 & 7.85 & 8.00 & 7.46 & 7.93 & 7.77 & 7.55 & 7.77 & 7.41 & 7.35 & 8.00 & 7.77 & 7.85 & 7.99 & 7.67 & 7.02 & 7.07 \\
\hline $5^{\prime}$ & 7.48 & 7.42 & 7.30 & 7.50 & 7.29 & 7.44 & 7.34 & 7.47 & 7.52 & 7.50 & 7.33 & 7.43 & 7.42 & 7.30 & 7.42 & 6.78 & 6.78 \\
\hline $6^{\prime}$ & 8.13 & 7.49 & 7.33 & 8.12 & 7.49 & 7.56 & 8.02 & 7.57 & 8.13 & 8.04 & 7.40 & 7.56 & 7.51 & 7.35 & 7.78 & 7.12 & 6.97 \\
\hline $7^{\prime}$ & 8.35 & 8.31 & 8.33 & 8.34 & 8.39 & 8.39 & 8.44 & 8.36 & 8.39 & 8.40 & 8.42 & 8.40 & 8.32 & 8.33 & 8.36 & 7.67 & 7.67 \\
\hline $8^{\prime}$ & 6.85 & 6.74 & 6.81 & 6.86 & 6.70 & 6.63 & 6.88 & 6.67 & 6.87 & 6.76 & 6.81 & 6.61 & 6.73 & 6.80 & 6.79 & 6.19 & 6.31 \\
\hline $2 "$ & 5.21 & 5.21 & 5.20 & 5.21 & 5.11 & 5.10 & 5.21 & 5.21 & 5.11 & 5.07 & 5.13 & 5.12 & 5.24 & 5.24 & 5.18 & 4.66 & 4.70 \\
\hline $3^{\prime \prime}$ & 4.01 & 4.08 & 4.08 & 4.02 & 4.11 & 4.11 & 4.02 & 4.08 & 4.02 & 4.09 & 4.04 & 4.23 & 4.24 & 4.24 & 4.06 & 3.59 & 3.66 \\
\hline \multirow[t]{2}{*}{ 4" } & 2.62 & 2.64 & 2.64 & 2.63 & 2.57 & 2.56 & 2.63 & 2.67 & 2.65 & 2.59 & 2.67 & 2.25 & 2.28 & 2.29 & 2.61 & 2.21 & 2.18 \\
\hline & 2.26 & 2.26 & 2.26 & 2.26 & 2.22 & 2.22 & 2.26 & 2.21 & 2.21 & 2.23 & 2.25 & 2.22 & 2.31 & 2.32 & 2.25 & 1.87 & 1.81 \\
\hline $3^{\prime \prime \prime}$ & 7.95 & 7.96 & 7.96 & 7.94 & 7.95 & 7.95 & 7.95 & 7.98 & 7.95 & 7.94 & 7.95 & 7.97 & 7.98 & 7.98 & 7.95 & 7.29 & 7.24 \\
\hline
\end{tabular}

${ }^{a}$ Calculated using the GIAO method at the mPWIPW91/6-311+G(2d,p) level in MeOH $(P C M) .{ }^{b}$ Measured in $\mathrm{CD}_{3} \mathrm{OD}(500 \mathrm{MHz}) .{ }^{c}$ Averaged according to the Boltzmann distribution theory at $298 \mathrm{~K}$ based on relative Gibbs free energies. ${ }^{d}$ Linearly corrected for the experimental data. 
Table S96. Calculated ${ }^{1} \mathrm{H}$ NMR chemical shifts of $\mathbf{4 B}$.

\begin{tabular}{|c|c|c|c|c|c|c|c|c|c|c|}
\hline \multirow[b]{2}{*}{ Position } & \multicolumn{9}{|c|}{ calculated $^{a}$} & \multirow{2}{*}{$\begin{array}{c}\text { experimental } \\
4\end{array}$} \\
\hline & 4B-1 & $4 \mathrm{~B}-2$ & $4 \mathrm{~B}-3$ & $4 \mathrm{~B}-4$ & 4B-5 & 4B-6 & 4B-7 & $\begin{array}{c}\text { averaged- } \\
\mathbf{4 B}^{c}\end{array}$ & $\begin{array}{l}\text { averaged-4B } \\
\text { (corrected) }^{d}\end{array}$ & \\
\hline 1 & 6.10 & 6.09 & 6.09 & 6.07 & 6.07 & 6.13 & 6.15 & 6.09 & 5.56 & 5.70 \\
\hline 2 & 3.99 & 3.99 & 4.11 & 4.10 & 4.12 & 4.00 & 4.02 & 4.04 & 3.64 & 3.64 \\
\hline 3 & 3.92 & 3.92 & 3.96 & 3.95 & 3.97 & 4.04 & 4.06 & 3.94 & 3.55 & 3.90 \\
\hline 4 & 5.55 & 5.56 & 5.57 & 5.56 & 5.57 & 5.59 & 5.59 & 5.56 & 5.06 & 4.93 \\
\hline 5 & 4.18 & 4.17 & 4.22 & 4.20 & 4.22 & 4.07 & 4.08 & 4.19 & 3.78 & 4.05 \\
\hline \multirow[t]{2}{*}{6} & 5.08 & 5.10 & 5.13 & 5.12 & 5.14 & 5.09 & 5.12 & 5.11 & 4.64 & 4.16 \\
\hline & 4.15 & 4.16 & 4.23 & 4.22 & 4.24 & 4.16 & 4.16 & 4.19 & 3.78 & 4.08 \\
\hline $2^{\prime}$ & 7.39 & 7.97 & 7.41 & 7.82 & 7.55 & 7.40 & 7.84 & 7.62 & 6.98 & 7.07 \\
\hline $5^{\prime}$ & 7.47 & 7.29 & 7.48 & 7.41 & 7.34 & 7.47 & 7.42 & 7.41 & 6.79 & 6.78 \\
\hline $6^{\prime}$ & 8.08 & 7.51 & 8.22 & 7.57 & 8.11 & 8.09 & 7.59 & 7.89 & 7.24 & 6.97 \\
\hline $7^{\prime}$ & 8.38 & 8.39 & 8.34 & 8.35 & 8.36 & 8.41 & 8.36 & 8.37 & 7.69 & 7.67 \\
\hline $8^{\prime}$ & 6.91 & 6.80 & 6.70 & 6.59 & 6.72 & 6.87 & 6.74 & 6.78 & 6.20 & 6.31 \\
\hline 2" & 4.90 & 4.89 & 4.92 & 4.90 & 4.91 & 5.05 & 5.07 & 4.91 & 4.45 & 4.70 \\
\hline 3" & 3.89 & 3.89 & 3.92 & 3.89 & 3.92 & 4.21 & 4.21 & 3.91 & 3.52 & 3.66 \\
\hline \multirow[t]{2}{*}{$4 "$} & 2.74 & 2.74 & 2.72 & 2.71 & 2.71 & 2.46 & 2.46 & 2.72 & 2.41 & 2.18 \\
\hline & 2.38 & 2.40 & 2.36 & 2.36 & 2.36 & 2.19 & 2.19 & 2.37 & 2.08 & 1.81 \\
\hline $3^{\prime \prime \prime}$ & 8.16 & 8.14 & 8.15 & 8.15 & 8.16 & 8.15 & 8.11 & 8.15 & 7.48 & 7.24 \\
\hline
\end{tabular}


Table S97. Calculated ${ }^{1} \mathrm{H}$ NMR chemical shifts of $\mathbf{4 C}$.

\begin{tabular}{|c|c|c|c|c|c|c|c|c|c|c|c|c|}
\hline \multirow[b]{2}{*}{ Position } & \multicolumn{11}{|c|}{ calculated $^{a}$} & \multirow{2}{*}{$\begin{array}{c}\text { experimental }^{b} \\
4\end{array}$} \\
\hline & $4 C-1$ & $4 \mathrm{C}-2$ & $4 \mathrm{C}-3$ & $4 \mathrm{C}-4$ & 4C-5 & 4C-6 & $4 \mathrm{C}-7$ & $4 C-8$ & 4C-9 & $\begin{array}{c}\text { averaged- } \\
\mathbf{4} \mathbf{C}^{c}\end{array}$ & $\begin{array}{c}\text { averaged-4C }^{(\text {corrected) }}{ }^{d}\end{array}$ & \\
\hline 1 & 6.14 & 6.14 & 6.15 & 6.16 & 6.16 & 6.17 & 6.19 & 6.17 & 6.20 & 6.15 & 5.66 & 5.70 \\
\hline 2 & 3.85 & 3.86 & 3.85 & 3.86 & 3.96 & 3.96 & 3.96 & 3.97 & 3.68 & 3.88 & 3.23 & 3.64 \\
\hline 3 & 4.77 & 4.77 & 4.76 & 4.77 & 4.77 & 4.77 & 4.77 & 4.77 & 4.10 & 4.76 & 4.17 & 3.90 \\
\hline 4 & 4.13 & 4.14 & 4.13 & 4.12 & 4.13 & 4.13 & 4.14 & 4.15 & 5.59 & 4.15 & 3.52 & 4.93 \\
\hline 5 & 5.15 & 5.16 & 5.13 & 5.14 & 5.18 & 5.18 & 5.19 & 5.19 & 4.23 & 5.14 & 4.58 & 4.05 \\
\hline \multirow[t]{2}{*}{6} & 5.30 & 5.30 & 5.28 & 5.28 & 5.32 & 5.32 & 5.30 & 5.31 & 5.14 & 5.30 & 4.74 & 4.16 \\
\hline & 4.15 & 4.16 & 4.14 & 4.14 & 4.17 & 4.17 & 4.16 & 4.17 & 4.27 & 4.15 & 3.52 & 4.08 \\
\hline $2^{\prime}$ & 7.79 & 7.96 & 7.36 & 7.55 & 8.01 & 7.84 & 7.40 & 7.54 & 7.61 & 7.72 & 7.34 & 7.07 \\
\hline $5^{\prime}$ & 7.46 & 7.32 & 7.46 & 7.32 & 7.29 & 7.41 & 7.49 & 7.35 & 7.35 & 7.39 & 6.98 & 6.78 \\
\hline $6^{\prime}$ & 7.64 & 7.55 & 8.06 & 7.96 & 7.43 & 7.56 & 8.21 & 8.11 & 7.95 & 7.75 & 7.37 & 6.97 \\
\hline $7^{\prime}$ & 8.39 & 8.40 & 8.33 & 8.40 & 8.33 & 8.32 & 8.33 & 8.35 & 8.40 & 8.37 & 8.03 & 7.67 \\
\hline $8^{\prime}$ & 6.76 & 6.82 & 6.89 & 6.93 & 6.69 & 6.61 & 6.72 & 6.73 & 6.90 & 6.80 & 6.35 & 6.31 \\
\hline 2" & 4.45 & 4.45 & 4.45 & 4.45 & 4.44 & 4.44 & 4.45 & 4.45 & 4.79 & 4.45 & 3.84 & 4.70 \\
\hline 3" & 2.74 & 2.74 & 2.74 & 2.74 & 2.70 & 2.70 & 2.70 & 2.70 & 3.26 & 2.74 & 2.01 & 3.66 \\
\hline \multirow[t]{2}{*}{$4^{\prime \prime}$} & 3.74 & 3.74 & 3.75 & 3.76 & 3.70 & 3.70 & 3.69 & 3.70 & 3.83 & 3.74 & 3.07 & 2.18 \\
\hline & 3.54 & 3.55 & 3.56 & 3.55 & 3.50 & 3.50 & 3.50 & 3.50 & 2.98 & 3.53 & 2.85 & 1.81 \\
\hline $3^{\prime \prime \prime}$ & 7.95 & 7.96 & 7.99 & 8.00 & 7.95 & 7.94 & 7.98 & 7.98 & 8.09 & 7.97 & 7.60 & 7.24 \\
\hline
\end{tabular}

${ }^{a}$ Calculated using the GIAO method at the mPWIPW91/6-311+G(2d,p) level in $\mathrm{MeOH}(\mathrm{PCM}) .{ }^{b}$ Measured in $\mathrm{CD}_{3} \mathrm{OD}(500 \mathrm{MHz}) .{ }^{c}$ Averaged according to the Boltzmann distribution theory at $298 \mathrm{~K}$ based on relative Gibbs free energies. ${ }^{d}$ Linearly corrected for the experimental data. 
Table S98. Calculated ${ }^{1} \mathrm{H}$ NMR chemical shifts of 4D.

\begin{tabular}{|c|c|c|c|c|c|c|c|c|c|c|c|c|c|c|c|c|c|c|}
\hline \multirow[b]{2}{*}{ Position } & \multicolumn{17}{|c|}{ calculated $^{a}$} & \multirow{2}{*}{$\begin{array}{c}\text { experimental }^{b} \\
4\end{array}$} \\
\hline & 4D-1 & 4D-2 & 4D-3 & $4 \mathrm{D}-4$ & 4D-5 & 4D-6 & 4D-7 & 4D-8 & 4D-9 & 4D-10 & 4D-11 & 4D-12 & 4D-13 & 4D-14 & 4D-15 & $\begin{array}{c}\text { averaged- } \\
\mathbf{4 D}^{c}\end{array}$ & $\begin{array}{c}\text { averaged-4D }^{\text {(corrected) }{ }^{d}}\end{array}$ & \\
\hline 1 & 6.23 & 6.21 & 6.12 & 6.25 & 6.25 & 6.11 & 6.15 & 6.14 & 6.15 & 6.10 & 6.15 & 6.17 & 6.15 & 6.16 & 6.10 & 6.18 & 5.68 & 5.70 \\
\hline 2 & 4.01 & 4.02 & 3.93 & 4.02 & 4.01 & 4.01 & 3.95 & 4.06 & 4.07 & 4.03 & 3.95 & 3.95 & 4.06 & 4.08 & 4.04 & 4.00 & 3.52 & 3.64 \\
\hline 3 & 4.29 & 4.29 & 4.02 & 4.29 & 4.29 & 4.05 & 4.02 & 4.26 & 4.28 & 4.07 & 4.02 & 4.02 & 4.26 & 4.28 & 4.07 & 4.19 & 3.71 & 3.90 \\
\hline 4 & 5.25 & 5.25 & 5.22 & 5.25 & 5.25 & 5.30 & 5.21 & 5.28 & 5.29 & 5.29 & 5.23 & 5.21 & 5.28 & 5.29 & 5.30 & 5.25 & 4.76 & 4.93 \\
\hline 5 & 4.41 & 4.40 & 4.28 & 4.41 & 4.41 & 4.27 & 4.29 & 4.37 & 4.37 & 4.29 & 4.28 & 4.29 & 4.38 & 4.37 & 4.29 & 4.35 & 3.87 & 4.05 \\
\hline \multirow[t]{2}{*}{6} & 4.99 & 4.99 & 4.80 & 5.00 & 5.00 & 4.79 & 4.82 & 4.98 & 4.97 & 4.83 & 4.81 & 4.82 & 4.99 & 4.97 & 4.83 & 4.92 & 4.43 & 4.16 \\
\hline & 4.21 & 4.21 & 4.23 & 4.21 & 4.21 & 4.23 & 4.22 & 4.20 & 4.21 & 4.22 & 4.23 & 4.22 & 4.20 & 4.21 & 4.22 & 4.22 & 3.73 & 4.08 \\
\hline $2^{\prime}$ & 7.38 & 7.85 & 7.81 & 8.01 & 7.56 & 7.86 & 7.35 & 7.83 & 7.42 & 7.42 & 7.96 & 7.56 & 7.98 & 7.61 & 7.57 & 7.67 & 7.14 & 7.07 \\
\hline $5^{\prime}$ & 7.43 & 7.39 & 7.44 & 7.23 & 7.34 & 7.38 & 7.45 & 7.40 & 7.43 & 7.48 & 7.25 & 7.37 & 7.30 & 7.29 & 7.32 & 7.39 & 6.87 & 6.78 \\
\hline $6^{\prime}$ & 8.18 & 7.66 & 7.65 & 7.61 & 8.08 & 7.64 & 8.12 & 7.60 & 8.21 & 8.20 & 7.55 & 8.02 & 7.52 & 8.12 & 8.11 & 7.88 & 7.35 & 6.97 \\
\hline $7^{\prime}$ & 8.30 & 8.39 & 8.38 & 8.41 & 8.36 & 8.25 & 8.32 & 8.34 & 8.28 & 8.25 & 8.38 & 8.36 & 8.34 & 8.31 & 8.29 & 8.34 & 7.81 & 7.67 \\
\hline $8^{\prime}$ & 6.96 & 6.88 & 6.81 & 6.93 & 6.97 & 6.59 & 6.99 & 6.62 & 6.72 & 6.67 & 6.94 & 6.99 & 6.68 & 6.74 & 6.69 & 6.85 & 6.33 & 6.31 \\
\hline $2^{\prime \prime}$ & 4.63 & 4.63 & 5.09 & 4.62 & 4.63 & 5.00 & 5.05 & 4.62 & 4.62 & 4.96 & 5.09 & 5.05 & 4.62 & 4.62 & 4.97 & 4.79 & 4.30 & 4.70 \\
\hline $3^{\prime \prime}$ & 2.87 & 2.90 & 2.81 & 2.90 & 2.87 & 2.85 & 2.85 & 2.86 & 2.83 & 2.89 & 2.81 & 2.85 & 2.86 & 2.83 & 2.89 & 2.86 & 2.39 & 3.66 \\
\hline \multirow[t]{2}{*}{ 4" } & 3.19 & 3.19 & 3.29 & 3.20 & 3.19 & 3.26 & 3.30 & 3.16 & 3.15 & 3.27 & 3.29 & 3.30 & 3.16 & 3.15 & 3.27 & 3.22 & 2.75 & 2.18 \\
\hline & 3.42 & 3.41 & 2.81 & 3.42 & 3.42 & 2.82 & 2.84 & 3.39 & 3.36 & 2.84 & 2.81 & 2.84 & 3.39 & 3.37 & 2.85 & 3.18 & 2.71 & 1.81 \\
\hline $3^{\prime \prime \prime}$ & 7.98 & 7.98 & 8.06 & 7.99 & 7.98 & 8.08 & 8.06 & 8.00 & 8.02 & 8.06 & 8.06 & 8.06 & 8.00 & 8.02 & 8.06 & 8.02 & 7.49 & 7.24 \\
\hline
\end{tabular}

${ }^{a}$ Calculated using the GIAO method at the mPWIPW91/6-311+G(2d,p) level in MeOH $(\mathrm{PCM}) .{ }^{b}$ Measured in $\mathrm{CD}_{3} \mathrm{OD}(500 \mathrm{MHz}) .{ }^{c}$ Averaged according to the Boltzmann distribution theory at $298 \mathrm{~K}$ based on relative Gibbs free energies. ${ }^{d}$ Linearly corrected for the experimental data. 
Table S99. Calculated ${ }^{13} \mathrm{C}$ NMR chemical shifts of $\mathbf{4 A}$.

\begin{tabular}{|c|c|c|c|c|c|c|c|c|c|c|c|c|c|c|c|c|c|}
\hline \multirow[b]{2}{*}{ Position } & \multicolumn{16}{|c|}{ calculated $^{a}$} & \multirow{2}{*}{$\begin{array}{c}\text { experimental }^{b} \\
4\end{array}$} \\
\hline & 4A-1 & $4 \mathrm{~A}-2$ & $4 \mathrm{~A}-3$ & $4 \mathrm{~A}-4$ & $4 \mathrm{~A}-5$ & $4 A-6$ & 4A-7 & 4A-8 & 4A-9 & 4A-10 & 4A-11 & $4 \mathrm{~A}-12$ & 4A-13 & $4 \mathrm{~A}-14$ & averaged-4A ${ }^{c}$ & $\begin{array}{l}\text { averaged-4A } \\
\text { (corrected) }^{d}\end{array}$ & \\
\hline 1 & 92.5 & 92.7 & 92.6 & 92.4 & 92.2 & 92.2 & 92.5 & 97.2 & 96.5 & 92.3 & 96.5 & 92.2 & 92.7 & 92.6 & 92.7 & 93.7 & 95.6 \\
\hline 2 & 70.3 & 70.5 & 70.5 & 70.4 & 70.2 & 70.3 & 70.3 & 74.8 & 75.1 & 70.2 & 75.1 & 70.3 & 70.5 & 70.5 & 70.6 & 72.4 & 74.1 \\
\hline 3 & 73.2 & 73.3 & 73.3 & 73.1 & 73.6 & 73.5 & 73.1 & 73.7 & 73.4 & 73.4 & 73.4 & 73.5 & 73.2 & 73.2 & 73.3 & 75.0 & 74.7 \\
\hline 4 & 74.9 & 74.9 & 74.8 & 74.9 & 74.8 & 74.8 & 74.9 & 73.4 & 73.4 & 74.8 & 73.6 & 74.8 & 74.9 & 74.9 & 74.8 & 76.4 & 76.9 \\
\hline 5 & 69.2 & 69.2 & 69.1 & 69.2 & 69.1 & 69.0 & 69.1 & 70.9 & 70.8 & 69.1 & 70.8 & 69.1 & 69.2 & 69.2 & 69.2 & 71.1 & 71.1 \\
\hline 6 & 65.4 & 65.4 & 65.4 & 65.4 & 65.4 & 65.4 & 65.4 & 65.4 & 65.4 & 65.4 & 65.3 & 65.3 & 65.3 & 65.4 & 65.4 & 67.4 & 67.2 \\
\hline $1^{\prime}$ & 125.3 & 124.8 & 126.7 & 125.7 & 127.2 & 125.9 & 126.8 & 125.2 & 125.0 & 125.0 & 126.5 & 125.7 & 124.8 & 126.7 & 125.7 & 125.5 & 127.5 \\
\hline $2^{\prime}$ & 119.3 & 110.3 & 112.4 & 119.3 & 112.2 & 110.3 & 121.0 & 110.4 & 119.3 & 119.2 & 112.4 & 110.5 & 110.3 & 112.5 & 115.3 & 115.4 & 115.3 \\
\hline $3^{\prime}$ & 144.9 & 145.5 & 148.0 & 144.3 & 147.4 & 145.1 & 146.4 & 144.9 & 144.8 & 144.1 & 147.3 & 145.1 & 145.5 & 148.1 & 145.7 & 144.7 & 146.8 \\
\hline $4^{\prime}$ & 151.1 & 152.4 & 150.3 & 151.1 & 150.4 & 152.1 & 149.0 & 152.3 & 151.8 & 151.1 & 149.6 & 152.2 & 152.4 & 150.3 & 151.1 & 149.9 & 149.9 \\
\hline $5^{\prime}$ & 116.6 & 114.9 & 113.6 & 116.5 & 114.0 & 114.9 & 115.4 & 115.6 & 116.6 & 115.9 & 114.3 & 114.8 & 114.9 & 113.6 & 115.4 & 115.5 & 116.5 \\
\hline $6^{\prime}$ & 122.1 & 130.1 & 127.8 & 122.2 & 129.1 & 130.5 & 120.7 & 131.1 & 122.4 & 121.9 & 128.8 & 130.5 & 130.1 & 127.8 & 125.8 & 125.5 & 123.3 \\
\hline $7^{\prime}$ & 152.7 & 152.8 & 152.9 & 152.5 & 151.3 & 151.2 & 153.1 & 153.7 & 154.2 & 151.2 & 154.6 & 151.2 & 152.8 & 152.9 & 152.6 & 151.3 & 148.7 \\
\hline $8^{\prime}$ & 108.8 & 108.5 & 109.2 & 109.0 & 111.8 & 111.1 & 110.0 & 110.6 & 109.5 & 112.1 & 109.1 & 111.2 & 108.5 & 109.1 & 109.5 & 109.8 & 113.9 \\
\hline $9^{\prime}$ & 170.9 & 171.0 & 170.9 & 170.7 & 168.6 & 168.6 & 170.7 & 173.2 & 174.9 & 168.4 & 175.0 & 168.7 & 170.9 & 170.9 & 170.6 & 168.7 & 167.5 \\
\hline 1" & 180.0 & 180.1 & 180.1 & 180.0 & 180.2 & 180.2 & 180.0 & 180.4 & 180.5 & 180.4 & 180.3 & 180.3 & 180.1 & 180.1 & 180.1 & 177.8 & 176.9 \\
\hline 2" & 48.3 & 48.3 & 48.3 & 48.3 & 48.5 & 48.5 & 48.3 & 48.1 & 47.7 & 48.4 & 47.7 & 48.9 & 48.5 & 48.5 & 48.3 & 50.9 & 44.5 \\
\hline 3" & 44.0 & 44.6 & 44.6 & 44.0 & 45.0 & 45.0 & 44.1 & 43.3 & 44.1 & 44.2 & 44.2 & 46.3 & 45.5 & 45.5 & 44.4 & 47.2 & 48.3 \\
\hline 4" & 27.0 & 27.0 & 27.0 & 27.0 & 27.3 & 27.3 & 27.0 & 27.6 & 27.4 & 27.5 & 27.3 & 29.1 & 29.2 & 29.2 & 27.1 & 30.6 & 31.2 \\
\hline 5" & 176.7 & 177.0 & 177.0 & 176.8 & 177.2 & 177.2 & 176.8 & 177.0 & 177.0 & 177.2 & 177.1 & 175.2 & 175.0 & 175.0 & 176.8 & 174.7 & 174.1 \\
\hline 6" & 178.1 & 178.2 & 178.2 & 178.2 & 178.4 & 178.3 & 178.2 & 178.2 & 177.9 & 178.2 & 177.9 & 178.5 & 178.5 & 178.4 & 178.2 & 176.0 & 173.5 \\
\hline 1"' & 117.5 & 117.4 & 117.4 & 117.5 & 117.8 & 117.8 & 117.5 & 116.6 & 116.6 & 117.9 & 116.5 & 117.7 & 117.4 & 117.4 & 117.5 & 117.5 & 118.1 \\
\hline 2"' & 120.3 & 120.3 & 120.3 & 120.3 & 120.4 & 120.4 & 120.3 & 120.3 & 120.5 & 120.4 & 120.6 & 120.3 & 120.2 & 120.2 & 120.3 & 120.3 & 117.4 \\
\hline 3"' & 115.3 & 115.3 & 115.3 & 115.3 & 115.2 & 115.2 & 115.3 & 114.8 & 115.6 & 115.4 & 115.4 & 115.2 & 115.3 & 115.3 & 115.3 & 115.4 & 114.8 \\
\hline 4"' & 147.6 & 148.0 & 148.0 & 147.8 & 148.4 & 148.4 & 147.7 & 148.4 & 148.5 & 148.4 & 148.6 & 148.6 & 148.1 & 148.1 & 148.0 & 146.9 & 148.3 \\
\hline $5^{\prime \prime \prime}$ & 133.7 & 134.2 & 134.2 & 133.8 & 135.0 & 135.0 & 133.7 & 135.5 & 135.4 & 135.1 & 136.0 & 135.2 & 134.5 & 134.5 & 134.2 & 133.6 & 136.5 \\
\hline 6"' & 141.8 & 142.3 & 142.3 & 142.1 & 143.2 & 143.2 & 141.9 & 142.8 & 142.1 & 143.2 & 142.5 & 143.8 & 143.0 & 143.0 & 142.3 & 141.4 & 143.3 \\
\hline 7"' & 168.8 & 168.7 & 168.7 & 168.8 & 168.5 & 168.5 & 168.8 & 168.3 & 168.3 & 168.6 & 168.2 & 168.3 & 168.5 & 168.6 & 168.7 & 166.8 & 165.7 \\
\hline
\end{tabular}

at $298 \mathrm{~K}$ based on relative Gibbs free energies. ${ }^{d}$ Linearly corrected for the experimental data. 
Table S100. Calculated ${ }^{13} \mathrm{C}$ NMR chemical shifts of $\mathbf{4 B}$.

\begin{tabular}{|c|c|c|c|c|c|c|c|c|c|c|}
\hline \multirow[b]{2}{*}{ Position } & \multicolumn{9}{|c|}{ calculated $^{a}$} & \multirow{2}{*}{$\frac{\text { experimental }^{b}}{4}$} \\
\hline & $4 \mathrm{~B}-1$ & $4 \mathrm{~B}-2$ & $4 \mathrm{~B}-3$ & $4 \mathrm{~B}-4$ & $4 \mathrm{~B}-5$ & $4 \mathrm{~B}-6$ & $4 \mathrm{~B}-7$ & averaged-4 $\mathbf{4} \mathbf{B}^{c}$ & $\begin{array}{l}\text { averaged-4B } \\
\text { (corrected) }^{d}\end{array}$ & \\
\hline 1 & 92.6 & 92.7 & 92.8 & 92.7 & 92.9 & 92.4 & 92.4 & 92.7 & 93.7 & 95.6 \\
\hline 2 & 71.1 & 71.1 & 71.0 & 71.1 & 71.0 & 70.7 & 70.8 & 71.0 & 73.0 & 74.1 \\
\hline 3 & 72.6 & 72.7 & 73.1 & 73.1 & 73.2 & 72.7 & 72.9 & 72.9 & 74.7 & 74.7 \\
\hline 4 & 73.4 & 73.4 & 73.5 & 73.5 & 73.5 & 74.9 & 75.0 & 73.5 & 75.3 & 76.9 \\
\hline 5 & 65.5 & 65.5 & 65.5 & 65.4 & 65.4 & 66.6 & 66.6 & 65.5 & 67.7 & 71.1 \\
\hline 6 & 64.3 & 64.3 & 64.3 & 64.3 & 64.3 & 63.8 & 63.8 & 64.3 & 66.5 & 67.2 \\
\hline $1^{\prime}$ & 125.9 & 127.5 & 125.8 & 125.8 & 127.6 & 125.6 & 125.5 & 126.3 & 126.0 & 127.5 \\
\hline $2^{\prime}$ & 119.3 & 112.2 & 119.9 & 110.5 & 120.9 & 119.4 & 110.3 & 116.4 & 116.4 & 115.3 \\
\hline $3^{\prime}$ & 144.6 & 147.4 & 143.9 & 145.4 & 146.4 & 144.7 & 145.2 & 145.4 & 144.2 & 146.8 \\
\hline $4^{\prime}$ & 151.4 & 149.4 & 150.7 & 152.0 & 147.8 & 151.4 & 152.1 & 150.6 & 149.2 & 149.9 \\
\hline $5^{\prime}$ & 116.0 & 113.8 & 116.5 & 115.1 & 115.1 & 116.1 & 114.8 & 115.4 & 115.5 & 116.5 \\
\hline $6^{\prime}$ & 121.5 & 128.5 & 122.1 & 130.4 & 120.1 & 121.6 & 130.3 & 124.7 & 124.4 & 123.3 \\
\hline $7^{\prime}$ & 152.8 & 152.9 & 151.1 & 151.4 & 151.3 & 152.9 & 152.8 & 152.1 & 150.7 & 148.7 \\
\hline $8^{\prime}$ & 110.6 & 109.7 & 113.0 & 112.2 & 113.5 & 110.2 & 108.9 & 111.4 & 111.6 & 113.9 \\
\hline $9^{\prime}$ & 170.7 & 170.6 & 169.2 & 169.2 & 169.3 & 170.7 & 170.7 & 170.0 & 167.8 & 167.5 \\
\hline 1" & 180.6 & 180.6 & 180.8 & 180.7 & 180.8 & 181.2 & 181.2 & 180.7 & 178.0 & 176.9 \\
\hline 2" & 50.2 & 50.0 & 49.5 & 49.4 & 49.5 & 51.0 & 50.8 & 49.9 & 52.7 & 44.5 \\
\hline 3" & 44.3 & 44.6 & 44.3 & 44.4 & 44.3 & 44.5 & 44.9 & 44.4 & 47.5 & 48.3 \\
\hline 4" & 27.3 & 27.3 & 27.5 & 27.4 & 27.4 & 29.4 & 29.5 & 27.4 & 31.2 & 31.2 \\
\hline $5^{\prime \prime}$ & 179.5 & 179.5 & 179.5 & 179.5 & 179.5 & 174.9 & 174.9 & 179.3 & 176.7 & 174.1 \\
\hline 6" & 177.7 & 177.7 & 177.7 & 177.6 & 177.6 & 178.3 & 178.4 & 177.7 & 175.2 & 173.5 \\
\hline 1"' & 117.4 & 117.5 & 117.3 & 117.3 & 117.3 & 118.0 & 118.2 & 117.4 & 117.4 & 118.1 \\
\hline 2"' & 121.3 & 121.3 & 120.5 & 120.6 & 120.5 & 120.8 & 120.9 & 121.0 & 120.8 & 117.4 \\
\hline 3"' & 116.5 & 116.3 & 116.4 & 116.4 & 116.4 & 116.0 & 115.8 & 116.4 & 116.5 & 114.8 \\
\hline 4"' & 147.6 & 147.6 & 147.4 & 147.5 & 147.4 & 147.7 & 147.7 & 147.5 & 146.3 & 148.3 \\
\hline 5"' & 134.5 & 134.4 & 134.3 & 134.2 & 134.4 & 134.9 & 134.7 & 134.4 & 133.7 & 136.5 \\
\hline 6"' & 142.8 & 142.9 & 142.7 & 142.7 & 142.7 & 142.7 & 142.7 & 142.8 & 141.7 & 143.3 \\
\hline 7"' & 169.4 & 169.4 & 169.3 & 169.2 & 169.3 & 168.7 & 168.6 & 169.3 & 167.1 & 165.7 \\
\hline
\end{tabular}

${ }^{a}$ Calculated using the GIAO method at the mPWIPW91/6-311+G(2d,p) level in MeOH (PCM). ${ }^{b}$ Measured in $C_{5} D_{5} N(125$ $\mathrm{MHz}$ ). ${ }^{\mathrm{c}}$ Averaged according to the Boltzmann distribution theory at $298 \mathrm{~K}$ based on relative Gibbs free energies. ${ }^{d}$ Linearly corrected for the experimental data. 
Table S101. Calculated ${ }^{13}$ C NMR chemical shifts of $\mathbf{4 C}$.

\begin{tabular}{|c|c|c|c|c|c|c|c|c|c|c|c|c|}
\hline \multirow[b]{2}{*}{ Position } & \multicolumn{11}{|c|}{ calculated $^{a}$} & \multirow{2}{*}{$\begin{array}{c}\text { experimental }^{b} \\
4 \\
\end{array}$} \\
\hline & $4 \mathrm{C}-1$ & $4 \mathrm{C}-2$ & $4 \mathrm{C}-3$ & $4 \mathrm{C}-4$ & $4 \mathrm{C}-5$ & $4 \mathrm{C}-6$ & $4 \mathrm{C}-7$ & $4 \mathrm{C}-8$ & 4C-9 & averaged-4 $\mathrm{C}^{c}$ & $\begin{array}{l}\text { averaged-4C } \\
\text { (corrected) }^{d}\end{array}$ & \\
\hline 1 & 93.1 & 93.2 & 93.0 & 93.1 & 93.1 & 93.0 & 93.1 & 93.2 & 96.7 & 93.2 & 93.8 & 95.6 \\
\hline 2 & 72.2 & 72.2 & 72.2 & 72.2 & 72.1 & 72.1 & 72.0 & 72.0 & 74.7 & 72.2 & 73.5 & 74.1 \\
\hline 3 & 70.3 & 70.3 & 70.2 & 70.2 & 70.7 & 70.7 & 70.7 & 70.7 & 73.2 & 70.4 & 71.7 & 74.7 \\
\hline 4 & 81.8 & 81.8 & 81.8 & 81.8 & 81.9 & 81.9 & 81.9 & 81.9 & 72.9 & 81.7 & 82.6 & 76.9 \\
\hline 5 & 64.1 & 64.1 & 64.1 & 64.1 & 64.5 & 64.5 & 64.6 & 64.5 & 68.3 & 64.3 & 65.8 & 71.1 \\
\hline 6 & 63.6 & 63.6 & 63.6 & 63.5 & 63.7 & 63.7 & 63.7 & 63.7 & 63.9 & 63.6 & 65.2 & 67.2 \\
\hline $1^{\prime}$ & 126.3 & 127.7 & 125.9 & 127.6 & 127.8 & 125.9 & 125.9 & 127.6 & 128.6 & 126.9 & 126.4 & 127.5 \\
\hline $2^{\prime}$ & 110.5 & 112.1 & 119.0 & 121.1 & 112.2 & 110.4 & 119.8 & 120.9 & 121.1 & 114.7 & 114.6 & 115.3 \\
\hline 3' & 144.8 & 147.2 & 144.9 & 147.0 & 147.5 & 145.3 & 143.9 & 146.4 & 146.8 & 146.0 & 144.8 & 146.8 \\
\hline $4^{\prime}$ & 151.6 & 149.2 & 151.4 & 149.0 & 149.1 & 151.9 & 150.7 & 147.9 & 148.8 & 150.3 & 149.0 & 149.9 \\
\hline $5^{\prime}$ & 115.1 & 113.9 & 115.8 & 114.5 & 113.7 & 115.1 & 116.5 & 115.1 & 115.1 & 114.8 & 114.7 & 116.5 \\
\hline $6^{\prime}$ & 130.2 & 128.6 & 121.2 & 119.3 & 128.1 & 130.3 & 122.1 & 120.2 & 120.6 & 125.9 & 125.4 & 123.3 \\
\hline $7^{\prime}$ & 152.6 & 152.9 & 152.6 & 153.1 & 151.4 & 151.2 & 151.0 & 151.2 & 154.3 & 152.4 & 151.1 & 148.7 \\
\hline $8^{\prime}$ & 109.9 & 110.5 & 110.7 & 111.5 & 113.2 & 112.5 & 113.2 & 113.6 & 111.4 & 111.2 & 111.2 & 113.9 \\
\hline $9^{\prime}$ & 170.5 & 170.6 & 170.7 & 170.7 & 169.2 & 169.2 & 169.1 & 169.3 & 174.0 & 170.3 & 168.4 & 167.5 \\
\hline 1" & 178.6 & 178.6 & 178.6 & 178.6 & 178.7 & 178.7 & 178.6 & 178.6 & 179.7 & 178.6 & 176.4 & 176.9 \\
\hline 2" & 47.1 & 47.2 & 47.3 & 47.3 & 46.8 & 46.8 & 46.8 & 46.8 & 46.7 & 47.1 & 49.2 & 44.5 \\
\hline 3" & 45.4 & 45.4 & 45.3 & 45.3 & 45.7 & 45.7 & 45.7 & 45.7 & 44.8 & 45.4 & 47.6 & 48.3 \\
\hline 4" & 31.3 & 31.3 & 31.3 & 31.3 & 31.4 & 31.4 & 31.3 & 31.3 & 30.4 & 31.3 & 33.9 & 31.2 \\
\hline $5^{\prime \prime}$ & 181.1 & 181.1 & 181.1 & 181.0 & 181.1 & 181.1 & 181.0 & 181.0 & 177.9 & 181.0 & 178.7 & 174.1 \\
\hline 6" & 175.8 & 175.8 & 175.8 & 175.8 & 175.8 & 175.8 & 175.9 & 175.9 & 181.0 & 175.9 & 173.8 & 173.5 \\
\hline 1"' & 118.8 & 118.8 & 118.9 & 119.0 & 118.6 & 118.6 & 118.7 & 118.7 & 123.1 & 118.9 & 118.6 & 118.1 \\
\hline 2"' & 122.2 & 122.2 & 122.0 & 121.9 & 121.4 & 121.4 & 121.4 & 121.4 & 119.5 & 121.9 & 121.5 & 117.4 \\
\hline 3"' & 115.2 & 115.2 & 115.4 & 115.4 & 115.0 & 115.0 & 115.1 & 115.1 & 115.7 & 115.2 & 115.1 & 114.8 \\
\hline 4"' & 148.3 & 148.3 & 148.3 & 148.3 & 147.7 & 147.7 & 147.5 & 147.5 & 147.6 & 148.1 & 146.9 & 148.3 \\
\hline $5^{\prime \prime \prime}$ & 133.7 & 133.8 & 134.2 & 134.2 & 134.1 & 134.1 & 134.6 & 134.6 & 134.5 & 134.0 & 133.3 & 136.5 \\
\hline 6"' & 141.9 & 142.0 & 142.3 & 142.3 & 142.2 & 142.2 & 142.6 & 142.6 & 142.4 & 142.1 & 141.1 & 143.3 \\
\hline $7^{7 \prime \prime}$ & 169.4 & 169.4 & 169.4 & 169.4 & 169.0 & 169.0 & 169.0 & 169.0 & 169.1 & 169.3 & 167.4 & 165.7 \\
\hline
\end{tabular}


Table S102. Calculated ${ }^{13} \mathrm{C}$ NMR chemical shifts of 4D.

\begin{tabular}{|c|c|c|c|c|c|c|c|c|c|c|c|c|c|c|c|c|c|c|}
\hline \multirow[b]{2}{*}{ Position } & \multicolumn{17}{|c|}{ calculated $^{a}$} & \multirow{2}{*}{$\frac{\text { experimental }^{b}}{4}$} \\
\hline & 4D-1 & 4D-2 & 4D-3 & 4D-4 & $4 \mathrm{D}-5$ & 4D-6 & 4D-7 & 4D-8 & 4D-9 & 4D-10 & 4D-11 & 4D-12 & 4D-13 & 4D-14 & 4D-15 & averaged-4D $\mathbf{D}^{c}$ & $\begin{array}{l}\text { averaged-4D } \\
\text { (corrected) }^{d}\end{array}$ & \\
\hline 1 & 92.2 & 92.4 & 92.8 & 92.3 & 92.2 & 92.6 & 92.7 & 92.0 & 92.1 & 92.7 & 92.7 & 92.7 & 92.0 & 92.1 & 92.8 & 92.4 & $\begin{array}{ll}92.8 \\
\end{array}$ & 95.6 \\
\hline 2 & 70.2 & 70.2 & 70.1 & 70.2 & 70.2 & 70.1 & 70.0 & 70.3 & 70.4 & 70.2 & 70.1 & 70.0 & 70.3 & 70.3 & 70.2 & 70.2 & 71.1 & 74.1 \\
\hline 3 & 72.9 & 73.0 & 71.8 & 73.0 & 72.9 & 71.9 & 71.8 & 73.0 & 73.1 & 72.0 & 71.8 & 71.8 & 73.0 & 73.1 & 72.0 & 72.5 & 73.4 & 74.7 \\
\hline 4 & 74.4 & 74.4 & 75.1 & 74.4 & 74.4 & 75.4 & 74.9 & 74.6 & 74.6 & 75.1 & 75.1 & 74.9 & 74.6 & 74.6 & 75.1 & 74.7 & 75.5 & 76.9 \\
\hline 5 & 67.9 & 68.0 & 68.0 & 68.0 & 68.0 & 68.0 & 68.0 & 67.9 & 67.9 & 67.9 & 68.0 & 68.0 & 67.9 & 67.9 & 67.9 & 68.0 & 69.0 & 71.1 \\
\hline 6 & 64.8 & 64.8 & 65.2 & 64.8 & 64.8 & 65.2 & 65.3 & 64.8 & 64.7 & 65.2 & 65.2 & 65.3 & 64.8 & 64.7 & 65.2 & 65.0 & 66.0 & 67.2 \\
\hline $1^{\prime}$ & 126.7 & 127.1 & 126.6 & 128.4 & 128.1 & 126.4 & 126.4 & 126.3 & 126.5 & 126.4 & 127.9 & 127.9 & 128.0 & 127.8 & 127.9 & 127.0 & 126.6 & 127.5 \\
\hline $2^{\prime}$ & 119.5 & 110.2 & 110.5 & 111.5 & 121.0 & 110.3 & 119.4 & 110.2 & 119.9 & 119.6 & 112.0 & 121.1 & 111.4 & 120.9 & 120.9 & 115.1 & 115.0 & 115.3 \\
\hline $3^{\prime}$ & 143.8 & 144.7 & 144.9 & 146.8 & 146.5 & 144.5 & 144.1 & 144.8 & 144.4 & 144.2 & 147.2 & 146.4 & 147.1 & 146.5 & 146.6 & 145.0 & 144.1 & 146.8 \\
\hline $4^{\prime}$ & 151.0 & 151.5 & 151.7 & 148.6 & 148.1 & 151.4 & 151.1 & 151.5 & 151.0 & 150.9 & 149.1 & 148.4 & 148.4 & 147.3 & 147.7 & 150.5 & 149.4 & 149.9 \\
\hline $5^{\prime}$ & 115.8 & 114.9 & 114.7 & 113.5 & 114.7 & 115.8 & 115.9 & 115.5 & 116.4 & 116.2 & 113.5 & 114.9 & 114.2 & 115.1 & 114.7 & 115.1 & 115.0 & 116.5 \\
\hline $6^{\prime}$ & 120.6 & 131.0 & 130.5 & 129.4 & 118.6 & 130.5 & 121.1 & 130.4 & 121.1 & 121.2 & 128.9 & 119.0 & 128.1 & 119.3 & 119.5 & 125.4 & 125.0 & 123.3 \\
\hline $7^{\prime}$ & 152.8 & 152.9 & 152.5 & 153.0 & 153.3 & 151.2 & 153.0 & 151.5 & 151.1 & 151.0 & 152.9 & 153.3 & 151.5 & 151.4 & 151.3 & 152.4 & 151.3 & 148.7 \\
\hline $8^{\prime}$ & 111.5 & 110.9 & 110.4 & 111.5 & 112.2 & 112.2 & 111.6 & 111.7 & 112.4 & 112.8 & 111.4 & 112.1 & 112.4 & 113.1 & 113.4 & 111.6 & 111.5 & 113.9 \\
\hline $9^{\prime}$ & 170.7 & 170.6 & 170.2 & 170.5 & 170.6 & 169.3 & 170.8 & 169.1 & 169.2 & 169.2 & 170.6 & 170.7 & 169.1 & 169.2 & 169.2 & 170.2 & 168.7 & 167.5 \\
\hline 1" & 177.5 & 177.5 & 184.6 & 177.6 & 177.5 & 184.6 & 184.8 & 177.7 & 177.7 & 184.8 & 184.6 & 184.8 & 177.7 & 177.7 & 184.8 & 180.3 & 178.5 & 176.9 \\
\hline 2" & 47.2 & 47.2 & 47.6 & 47.2 & 47.2 & 47.2 & 47.6 & 47.5 & 47.2 & 47.4 & 47.6 & 47.7 & 47.5 & 47.2 & 47.4 & 47.3 & 48.8 & 44.5 \\
\hline 3" & 47.7 & 47.7 & 47.9 & 47.7 & 47.7 & 47.8 & 47.7 & 47.9 & 48.0 & 47.7 & 47.9 & 47.7 & 47.9 & 48.0 & 47.7 & 47.8 & 49.3 & 48.3 \\
\hline 4" & 32.9 & 32.8 & 33.6 & 32.8 & 32.8 & 34.0 & 33.9 & 32.9 & 33.0 & 34.4 & 33.6 & 33.9 & 32.9 & 33.0 & 34.4 & 33.2 & 35.1 & 31.2 \\
\hline 5" & 179.5 & 179.5 & 179.1 & 179.5 & 179.5 & 179.1 & 179.0 & 179.6 & 179.5 & 179.0 & 179.1 & 179.0 & 179.6 & 179.5 & 179.0 & 179.4 & 177.6 & 174.1 \\
\hline $6^{\prime \prime}$ & 174.7 & 174.8 & 173.9 & 174.7 & 174.7 & 174.3 & 174.1 & 174.6 & 174.8 & 174.5 & 174.0 & 174.0 & 174.6 & 174.8 & 174.5 & 174.5 & 172.9 & 173.5 \\
\hline $1^{\prime \prime \prime}$ & 119.3 & 119.4 & 119.4 & 119.4 & 119.3 & 119.9 & 119.2 & 119.1 & 119.1 & 119.8 & 119.4 & 119.2 & 119.1 & 119.1 & 119.8 & 119.4 & 119.1 & 118.1 \\
\hline $2^{\prime \prime \prime}$ & 120.5 & 120.4 & 120.8 & 120.5 & 120.5 & 120.9 & 120.9 & 120.4 & 120.4 & 121.2 & 120.8 & 120.9 & 120.4 & 120.4 & 121.2 & 120.6 & 120.3 & 117.4 \\
\hline 3"' & 115.8 & 115.8 & 116.6 & 115.8 & 115.8 & 117.0 & 116.6 & 116.1 & 116.1 & 117.1 & 116.6 & 116.6 & 116.1 & 116.1 & 117.1 & 116.2 & 116.0 & 114.8 \\
\hline 4"' & 147.8 & 147.9 & 148.4 & 147.9 & 147.8 & 148.0 & 148.6 & 147.8 & 147.7 & 147.8 & 148.4 & 148.6 & 147.9 & 147.7 & 147.8 & 148.0 & 147.0 & 148.3 \\
\hline $5^{\prime \prime \prime}$ & 134.5 & 134.7 & 133.7 & 134.7 & 134.5 & 133.8 & 134.2 & 134.7 & 134.7 & 134.2 & 133.7 & 134.2 & 134.7 & 134.7 & 134.2 & 134.3 & 133.7 & 136.5 \\
\hline 6"' & 143.3 & 143.5 & 141.1 & 143.5 & 143.3 & 141.4 & 141.6 & 142.9 & 142.6 & 141.8 & 141.1 & 141.6 & 142.9 & 142.7 & 141.8 & 142.5 & 141.7 & 143.3 \\
\hline 7"' & 169.0 & 169.0 & 168.5 & 169.0 & 169.0 & 168.5 & 168.4 & 168.9 & 168.8 & 168.6 & 168.5 & 168.5 & 168.9 & 168.8 & 168.6 & 168.8 & 167.3 & 165.7 \\
\hline
\end{tabular}

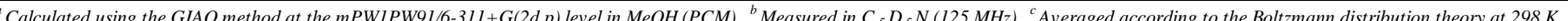
based on relative Gibbs free energies. ${ }^{d}$ Linearly corrected for the experimental data. 
Figure S13. Correlation plots of experimental ${ }^{1} \mathrm{H}$ NMR chemical shifts versus corresponding calculated ${ }^{1} \mathrm{H}$ NMR chemical shifts of 4 .
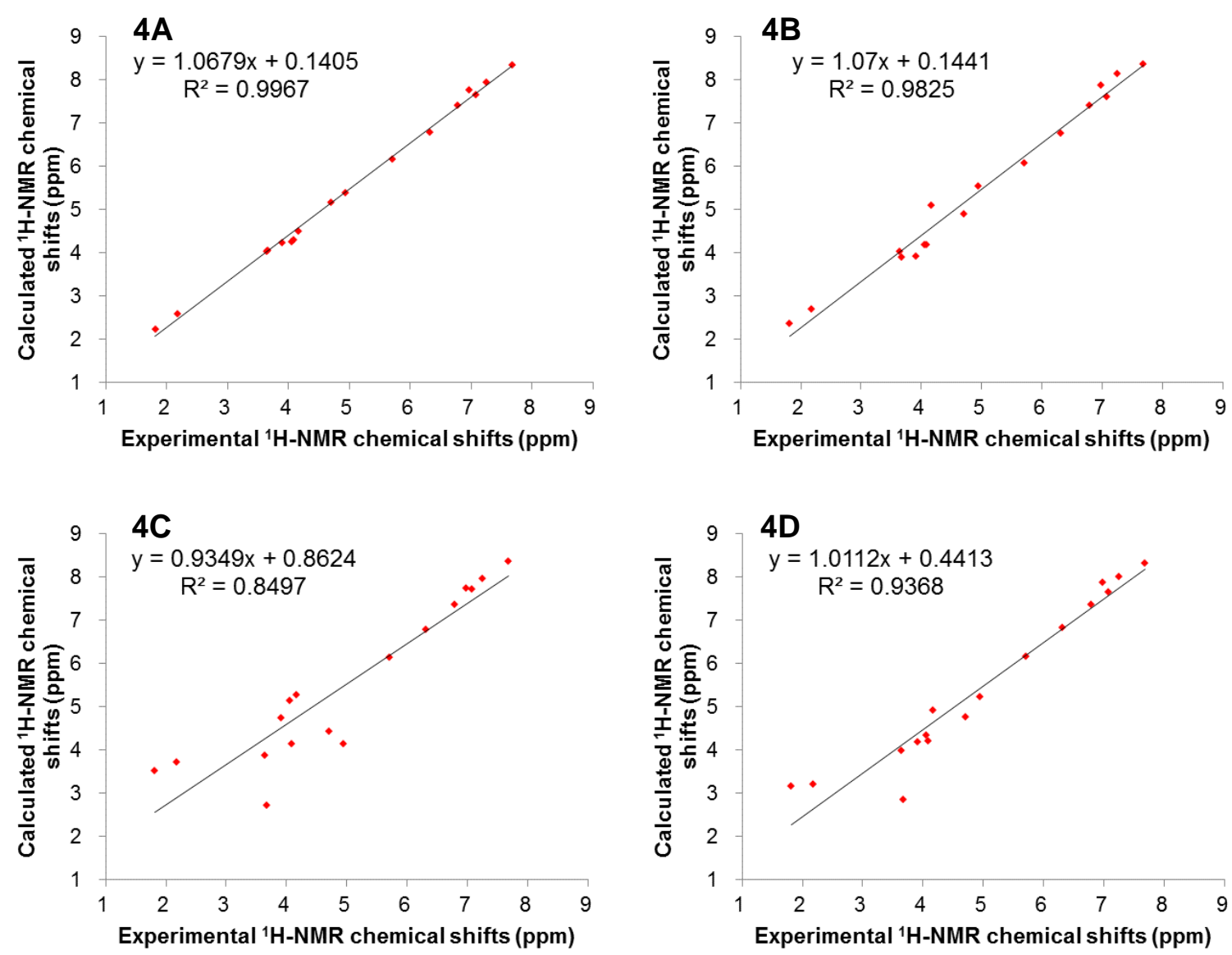
Figure S14. Correlation plots of experimental ${ }^{13} \mathrm{C}$ NMR chemical shifts versus corresponding calculated ${ }^{13} \mathrm{C}$ NMR chemical shifts of 4 .
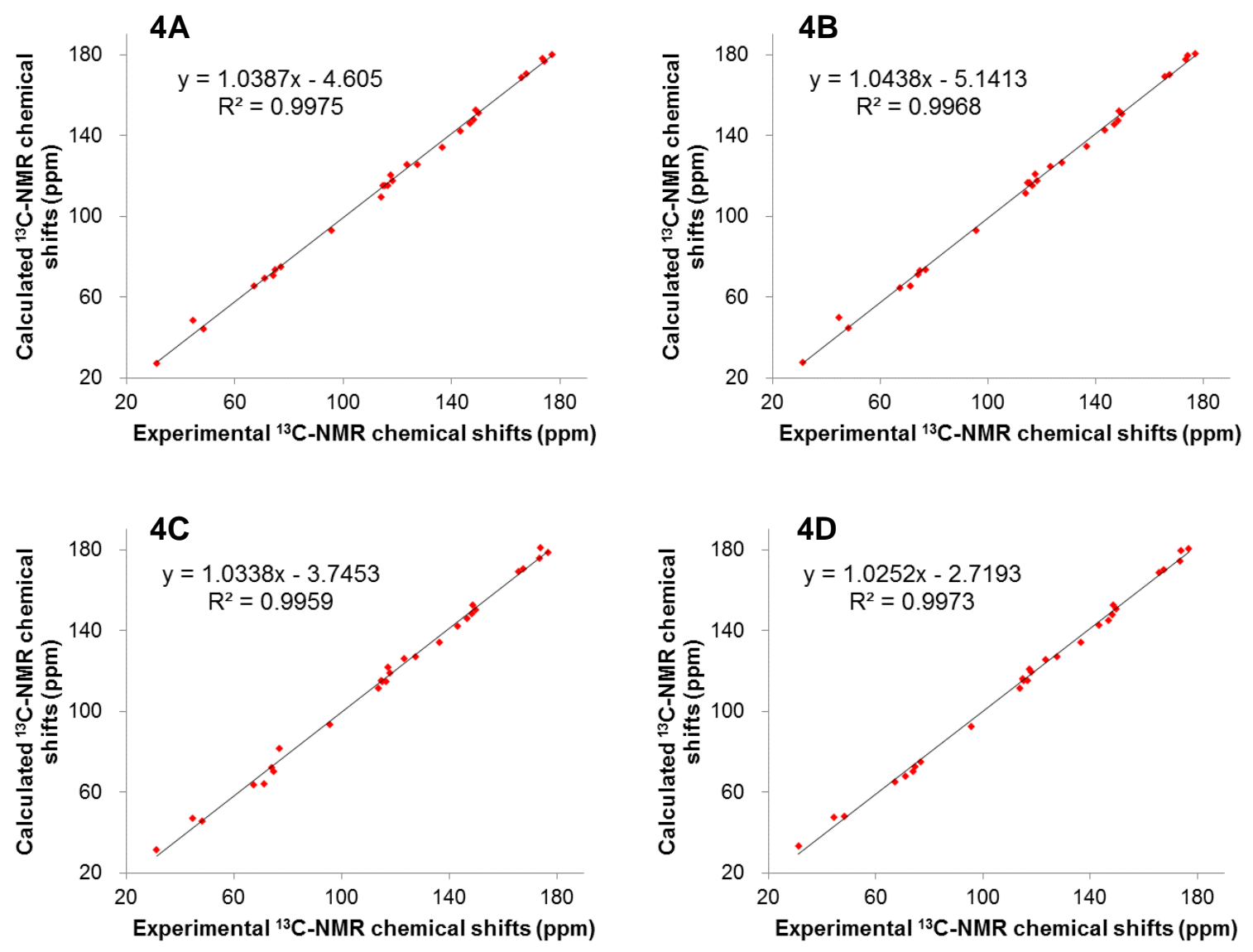
Table S103. Statistical parameters of calculated ${ }^{1} \mathrm{H}$ NMR chemical shifts of 4 (ppm).

\begin{tabular}{rccc}
\hline & $R^{2}$ & CMaxErr & CMAE \\
\hline 4A & $\mathbf{0 . 9 9 6 7}$ & $\mathbf{0 . 1 9}$ & $\mathbf{0 . 0 8}$ \\
4B & 0.9825 & 0.48 & 0.19 \\
4C & 0.8497 & 1.65 & 0.58 \\
4D & 0.9368 & 1.27 & 0.32 \\
\hline
\end{tabular}

$R^{2}$ : coefficient of determination; CMaxErr: corrected maximum absolute error with respect to the linear fit; CMAE:

corrected mean absolute error with respect to the linear fit

Table S104. Statistical parameters of calculated ${ }^{13} \mathrm{C}$ NMR chemical shifts of 4 (ppm).

\begin{tabular}{rccc}
\hline & $R^{2}$ & CMaxErr & CMAE \\
\hline 4A & $\mathbf{0 . 9 9 7 5}$ & 6.4 & $\mathbf{1 . 6}$ \\
4B & 0.9968 & 8.2 & 1.8 \\
4C & 0.9959 & 5.7 & 2.1 \\
4D & 0.9973 & $\mathbf{4 . 3}$ & 1.9 \\
\hline
\end{tabular}

$R^{2}$ : coefficient of determination; CMaxErr: corrected maximum absolute error with respect to the linear fit; CMAE.

corrected mean absolute error with respect to the linear fit 
Table S105. Calculated ${ }^{1} \mathrm{H}$ NMR coupling constants between $\mathrm{H}-2$ " and $\mathrm{H}-3^{\prime \prime}$ in $\mathbf{4 A}$ (ppm).

\begin{tabular}{|c|c|c|c|c|c|c|c|c|c|c|c|c|c|c|c|c|c|}
\hline & \multicolumn{16}{|c|}{ calculated $^{a}$} & \multirow{2}{*}{$\begin{array}{c}\text { experimental }^{b} \\
4\end{array}$} \\
\hline & 4A-1 & 4A-2 & 4A-3 & $4 \mathrm{~A}-4$ & 4A-5 & $4 A-6$ & 4A-7 & 4A-8 & 4A-9 & $4 \mathrm{~A}-10$ & 4A-11 & 4A-12 & 4A-13 & 4A-14 & $\begin{array}{c}\text { averaged- } \\
\mathbf{4 A}^{c} \\
\end{array}$ & $\begin{array}{l}\text { averaged-4A } \\
\text { (corrected) }^{d}\end{array}$ & \\
\hline$J_{2^{\prime \prime}-3^{\prime \prime}}$ & 2.6 & 2.5 & 2.6 & 2.6 & 2.5 & 2.5 & 2.6 & 2.5 & 2.5 & 2.5 & 2.5 & 2.3 & 2.4 & 2.4 & 2.5 & 2.4 & 2 \\
\hline
\end{tabular}

${ }^{a}$ Calculated at the B3LYP/6-31G(d,p)u+1s (using only the Fermi contact term) level in MeOH $(P C M) .{ }^{b}$ Measured in $\mathrm{CD}_{3} \mathrm{OD}(500 \mathrm{MHz}) .{ }^{c}$ Averaged according to the Boltzmann distribution theory at 298 K based on relative Gibbs free energies. ${ }^{d}$ Scaled by using the slope parameter 0.94.

Table S106. Calculated ${ }^{1} \mathrm{H}$ NMR coupling constants between H-2" and H-3" in 4B (ppm).

\begin{tabular}{|c|c|c|c|c|c|c|c|c|c|c|}
\hline & \multicolumn{9}{|c|}{ calculated $^{a}$} & \multirow{2}{*}{$\begin{array}{c}\text { experimental } \\
4\end{array}$} \\
\hline & $4 B-1$ & 4B-2 & $4 B-3$ & $4 B-4$ & 4B-5 & $4 B-6$ & $4 B-7$ & $\begin{array}{c}\text { averaged- } \\
\mathbf{4 B}^{c}\end{array}$ & $\begin{array}{l}\text { averaged-4B } \\
{\text { (corrected) }{ }^{d}}^{\text {a }}\end{array}$ & \\
\hline$J_{2^{\prime \prime}-3^{n}}$ & 1.7 & 1.7 & 1.7 & 1.7 & 1.7 & 1.5 & 1.5 & 1.7 & 1.6 & 2 \\
\hline
\end{tabular}

${ }^{a}$ Calculated at the B3LYP/6-31G(d,p)u+1s (using only the Fermi contact term) level in MeOH (PCM). ${ }^{b}$ Measured in $\mathrm{CD}_{3} \mathrm{OD}$

$(500 \mathrm{MHz}) .{ }^{c}$ Averaged according to the Boltzmann distribution theory at $298 \mathrm{~K}$ based on relative Gibbs free energies. ${ }^{d}$ Scaled by using the slope parameter 0.94

Table S107. Calculated ${ }^{1} \mathrm{H}$ NMR coupling constants between $\mathrm{H}-2^{\prime \prime}$ and $\mathrm{H}-3^{\prime \prime}$ in 4C (ppm).

\begin{tabular}{|c|c|c|c|c|c|c|c|c|c|c|c|c|}
\hline & \multicolumn{11}{|c|}{ calculated $^{a}$} & \multirow{2}{*}{$\begin{array}{c}\text { experimental } \\
4\end{array}$} \\
\hline & $4 C-1$ & $4 C-2$ & $4 C-3$ & $4 C-4$ & $4 C-5$ & $4 C-6$ & $4 C-7$ & $4 C-8$ & 4C-9 & $\begin{array}{c}\text { averaged- } \\
\mathbf{4 C}^{c}\end{array}$ & $\begin{array}{l}\text { averaged-4C } \\
{\text { (corrected) }{ }^{d}}^{\text {cor }}\end{array}$ & \\
\hline$J_{2^{\prime \prime}-3 "}$ & 12.5 & 12.5 & 12.5 & 12.5 & 12.4 & 12.4 & 12.4 & 12.4 & 6.1 & 12.4 & 11.6 & 2 \\
\hline
\end{tabular}

${ }^{a}$ Calculated at the B3LYP/6-31G(d p)u+1s (using only the Fermi contact term) level in MeOH (PCM) ${ }^{b}$ Measured in $C D$ OD (5OO MHz).

Averaged according to the Boltzmann distribution theory at $298 \mathrm{~K}$ based on relative Gibbs free energies. ${ }^{d}$ Scaled by using the slope parameter 0.94. 
Table S108. Calculated ${ }^{1} \mathrm{H}$ NMR coupling constants between H-2" and H-3" in 4D (ppm).

\begin{tabular}{|c|c|c|c|c|c|c|c|c|c|c|c|c|c|c|c|c|c|c|}
\hline & \multicolumn{17}{|c|}{ calculated $^{a}$} & \multirow{2}{*}{$\begin{array}{c}\text { experimental }^{b} \\
4 \\
\end{array}$} \\
\hline & 4D-1 & 4D-2 & 4D-3 & $4 \mathrm{D}-4$ & 4D-5 & 4D-6 & 4D-7 & $4 \mathrm{D}-8$ & 4D-9 & 4D-10 & 4D-11 & 4D-12 & 4D-13 & 4D-14 & 4D-15 & $\begin{array}{c}\text { averaged- } \\
\mathbf{4} \mathbf{D}^{c} \\
\end{array}$ & $\begin{array}{l}\text { averaged-4D } \\
\text { (corrected) }^{d}\end{array}$ & \\
\hline$J_{2^{\prime \prime}-3^{n}}$ & 11.7 & 11.6 & 11.8 & 11.7 & 11.7 & 11.7 & 11.8 & 11.7 & 11.7 & 11.9 & 11.8 & 11.8 & 11.7 & 11.7 & 11.9 & 11.7 & 11.0 & 2 \\
\hline
\end{tabular}


Figure $\mathrm{S} 15 .{ }^{1} \mathrm{H}$ and ${ }^{13} \mathrm{C}$ NMR (500 MHz for ${ }^{1} \mathrm{H}$ and $125 \mathrm{MHz}$ for ${ }^{13} \mathrm{C}, \mathrm{CD}_{3} \mathrm{OD}$ ) spectra of $\mathbf{1}$.
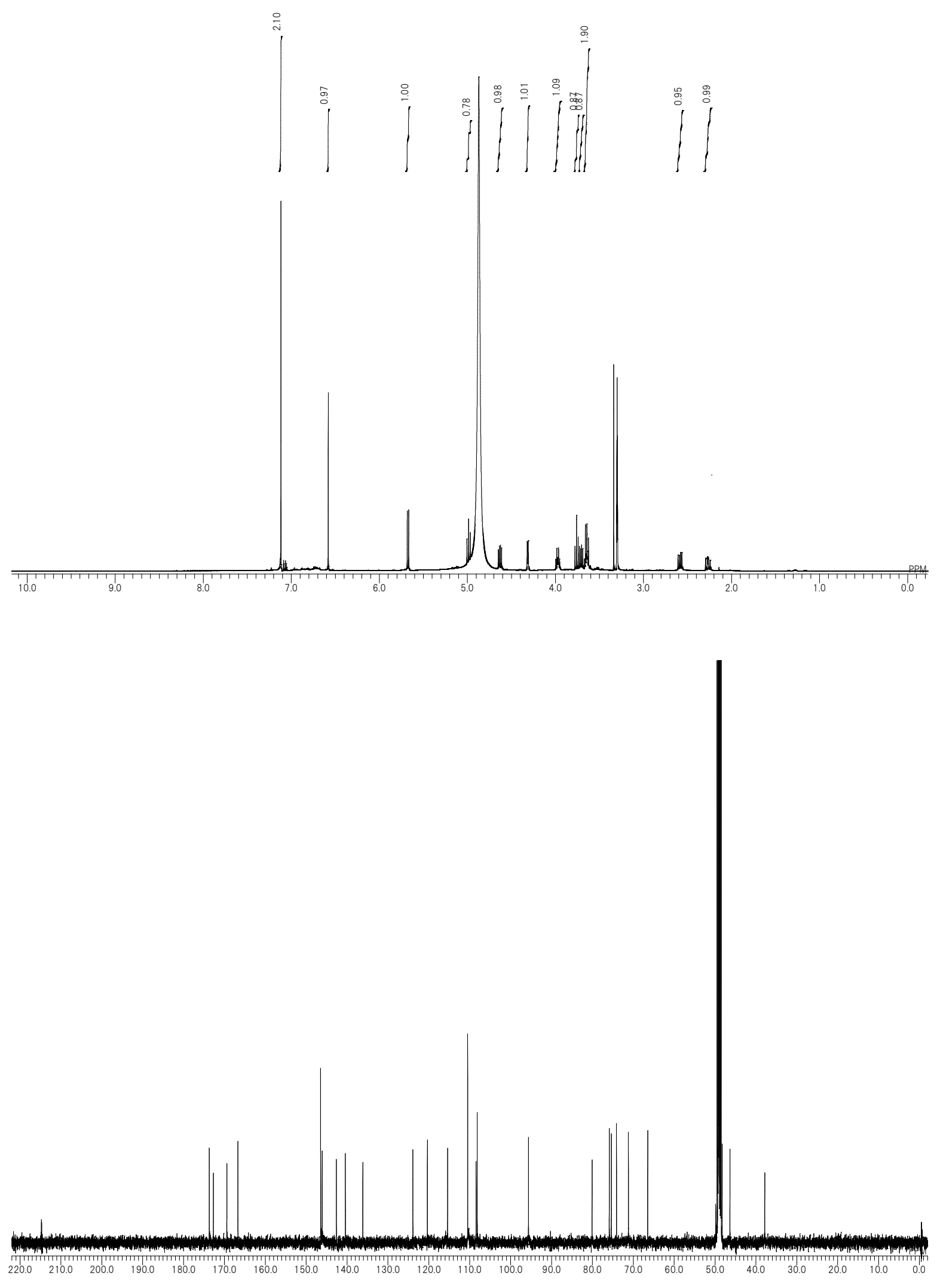
Figure S16. HMBC (500 MHz, CD $\mathrm{CDD}_{3} \mathrm{O}$ ) spectrum of $\mathbf{1}$.

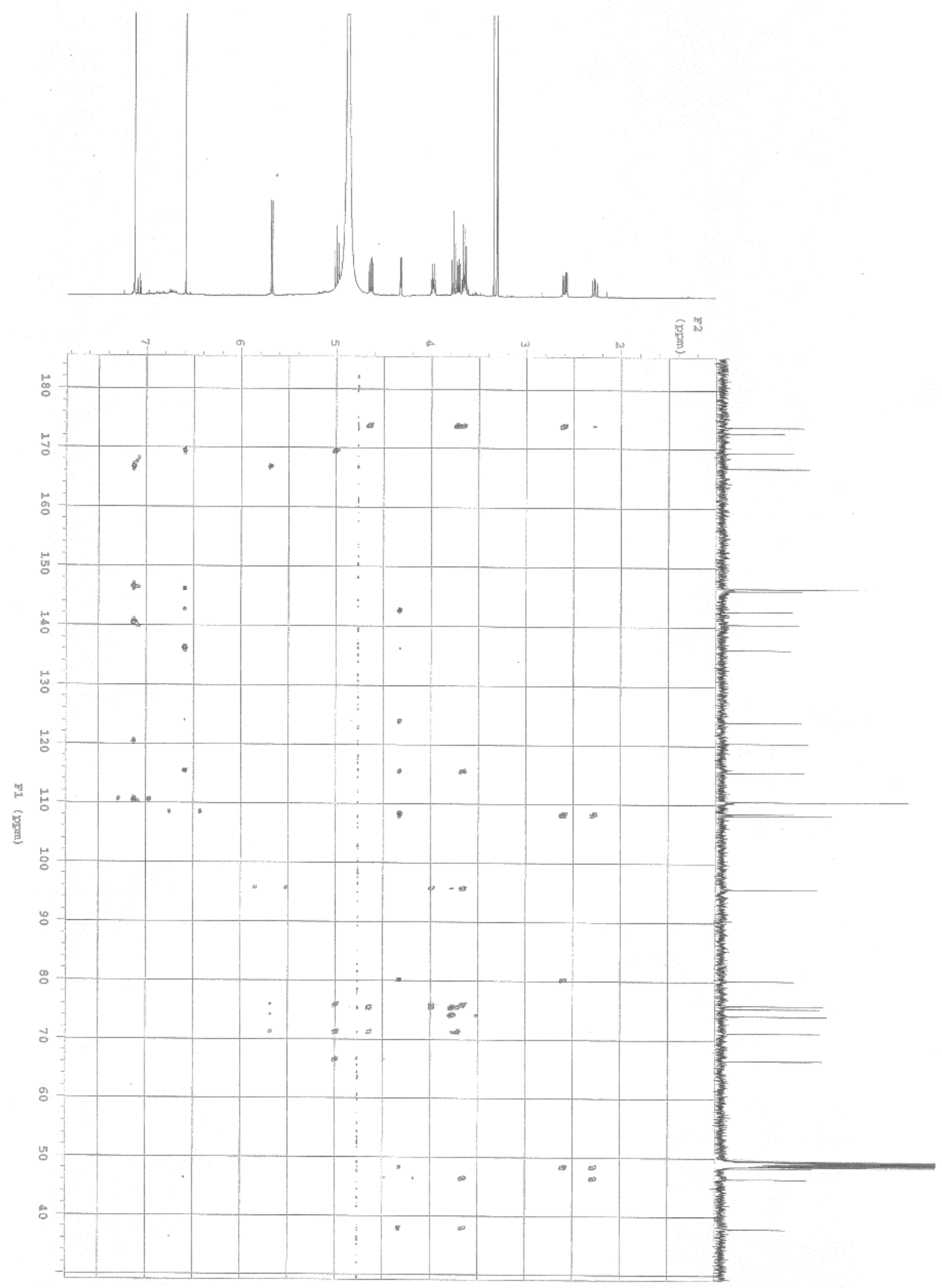

S87 
Figure S17. COSY (500 MHz, $\mathrm{CD}_{3} \mathrm{OD}$ ) spectrum of $\mathbf{1}$.

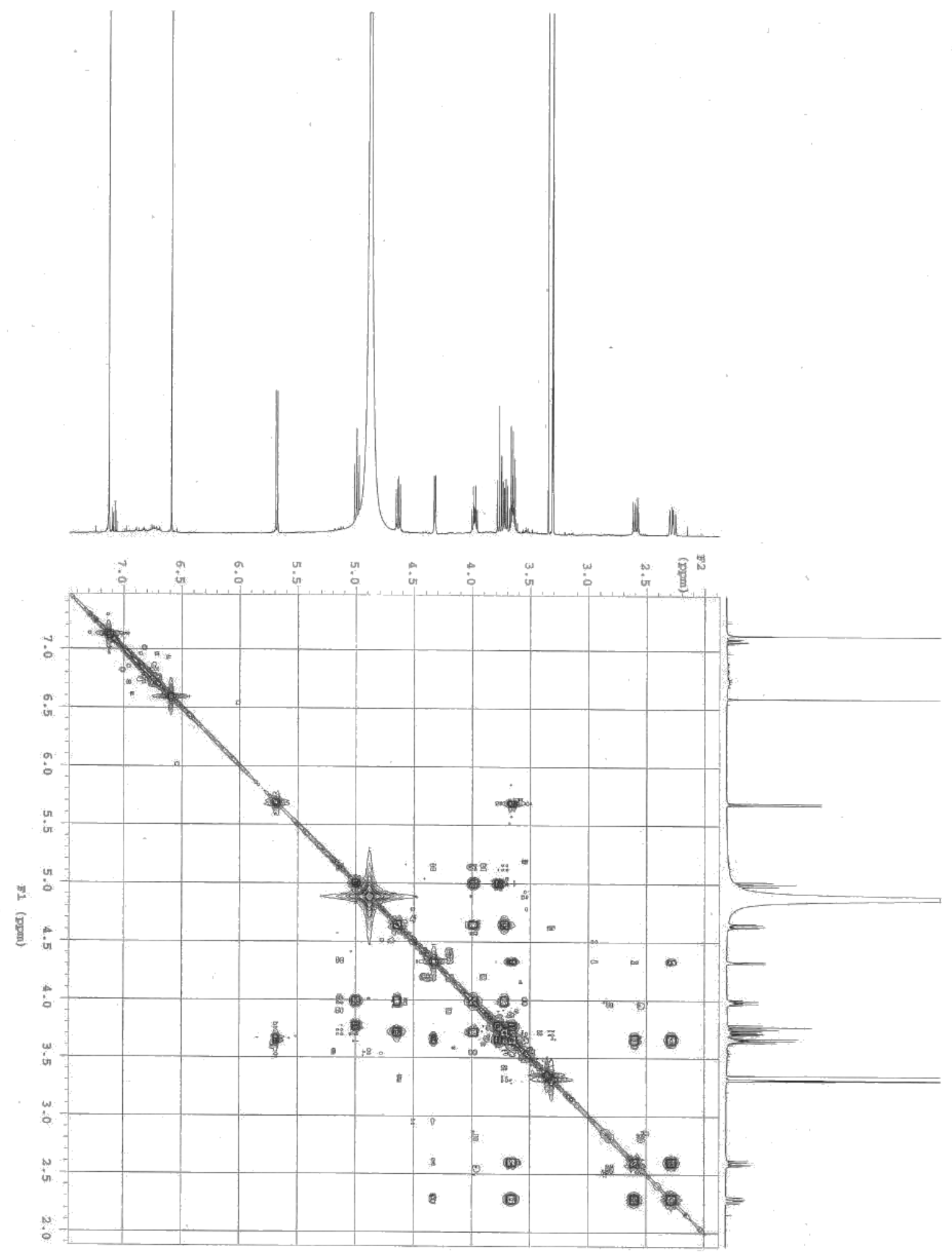


Figure S18. NOESY (500 MHz, CD 30 D) spectrum of 1.

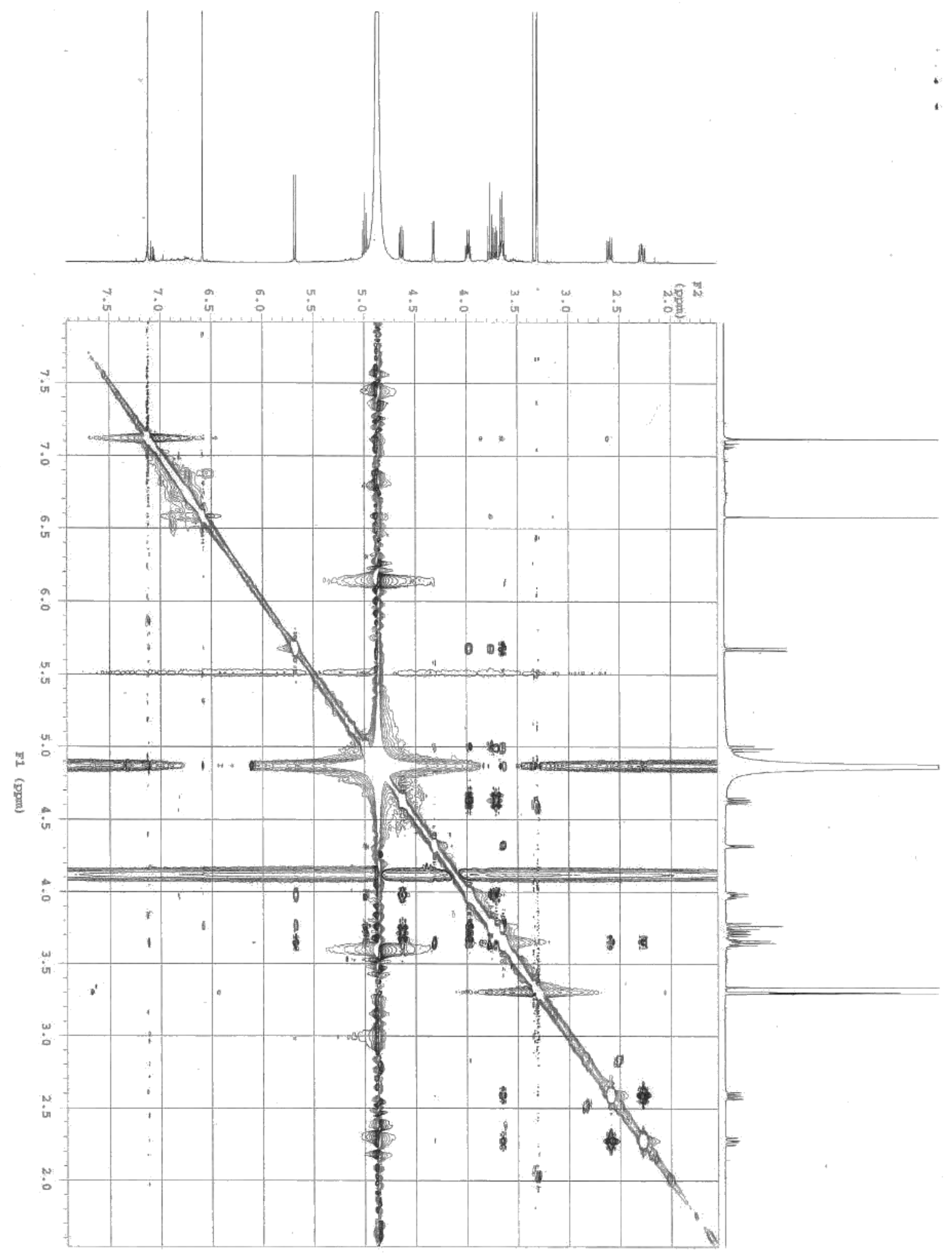


Figure S19. ${ }^{1} \mathrm{H}$ and ${ }^{13} \mathrm{C}$ NMR (500 MHz for ${ }^{1} \mathrm{H}$ and $125 \mathrm{MHz}$ for ${ }^{13} \mathrm{C}, \mathrm{CD}_{3} \mathrm{OD}$ ) spectra of 2.
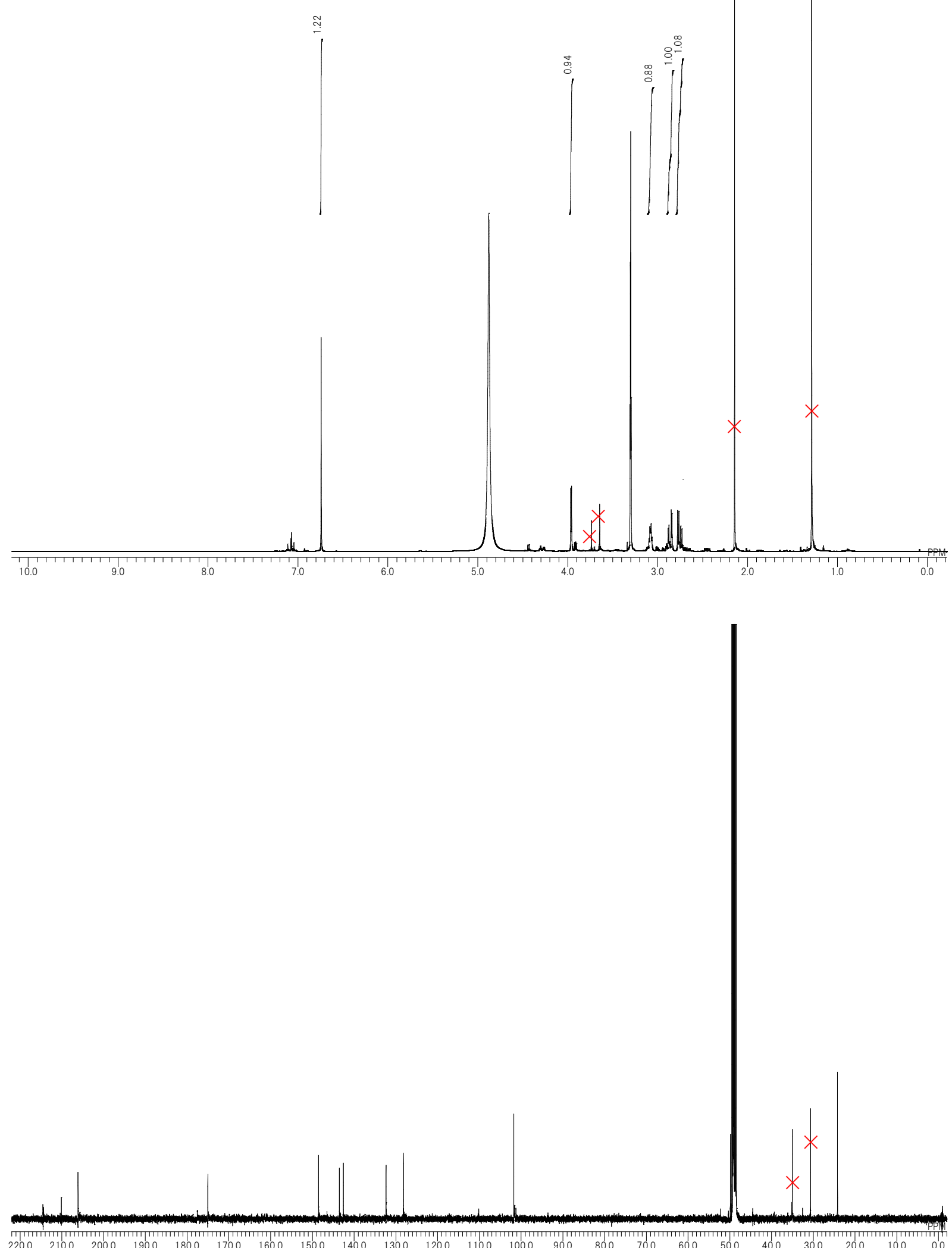
Figure S20. HMBC (500 MHz, $\left.\mathrm{CD}_{3} \mathrm{OD}\right)$ spectrum of 2.

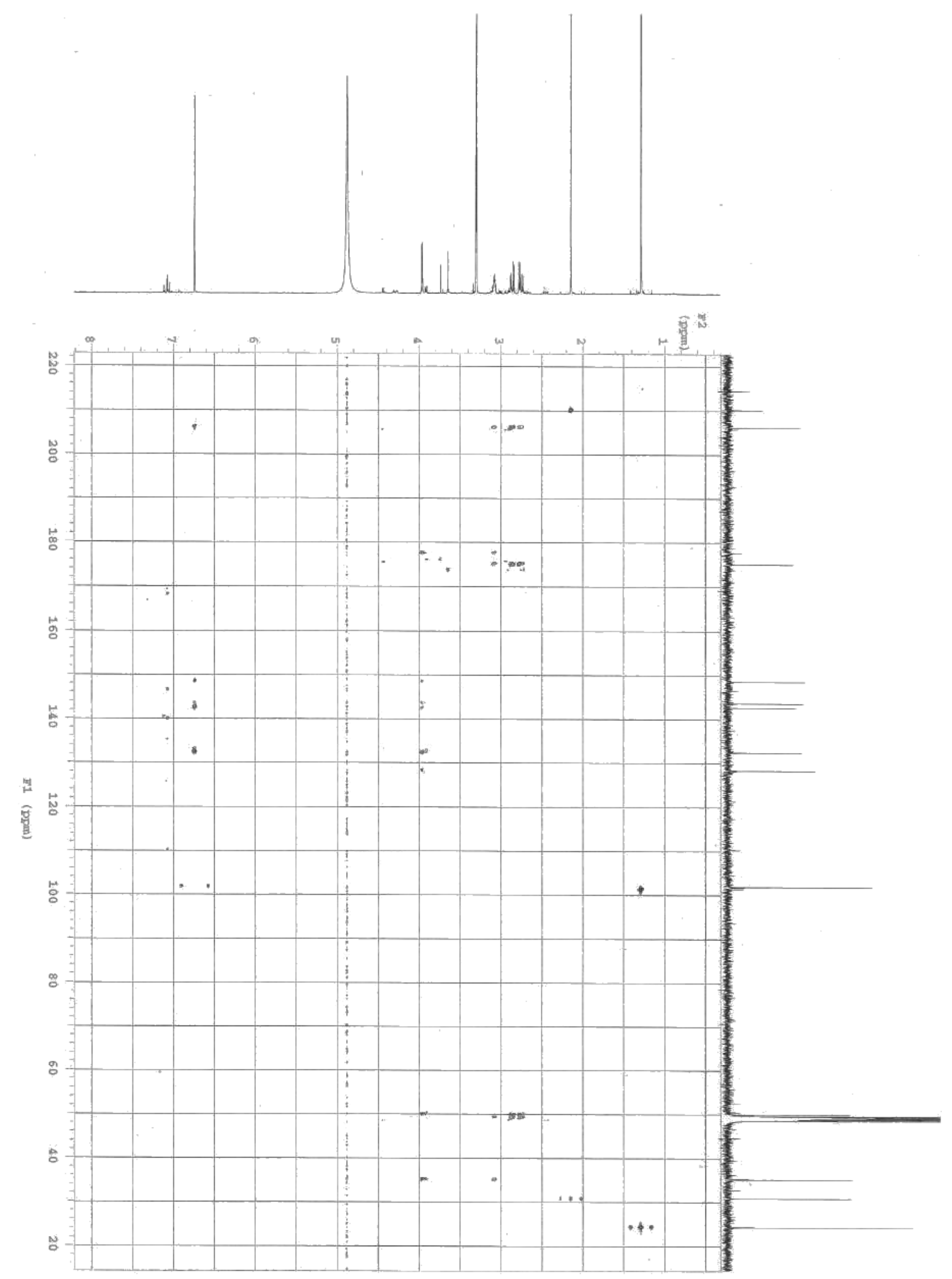


Figure S21. COSY (500 MHz, CD $\left.{ }_{3} \mathrm{OD}\right)$ spectrum of 2.

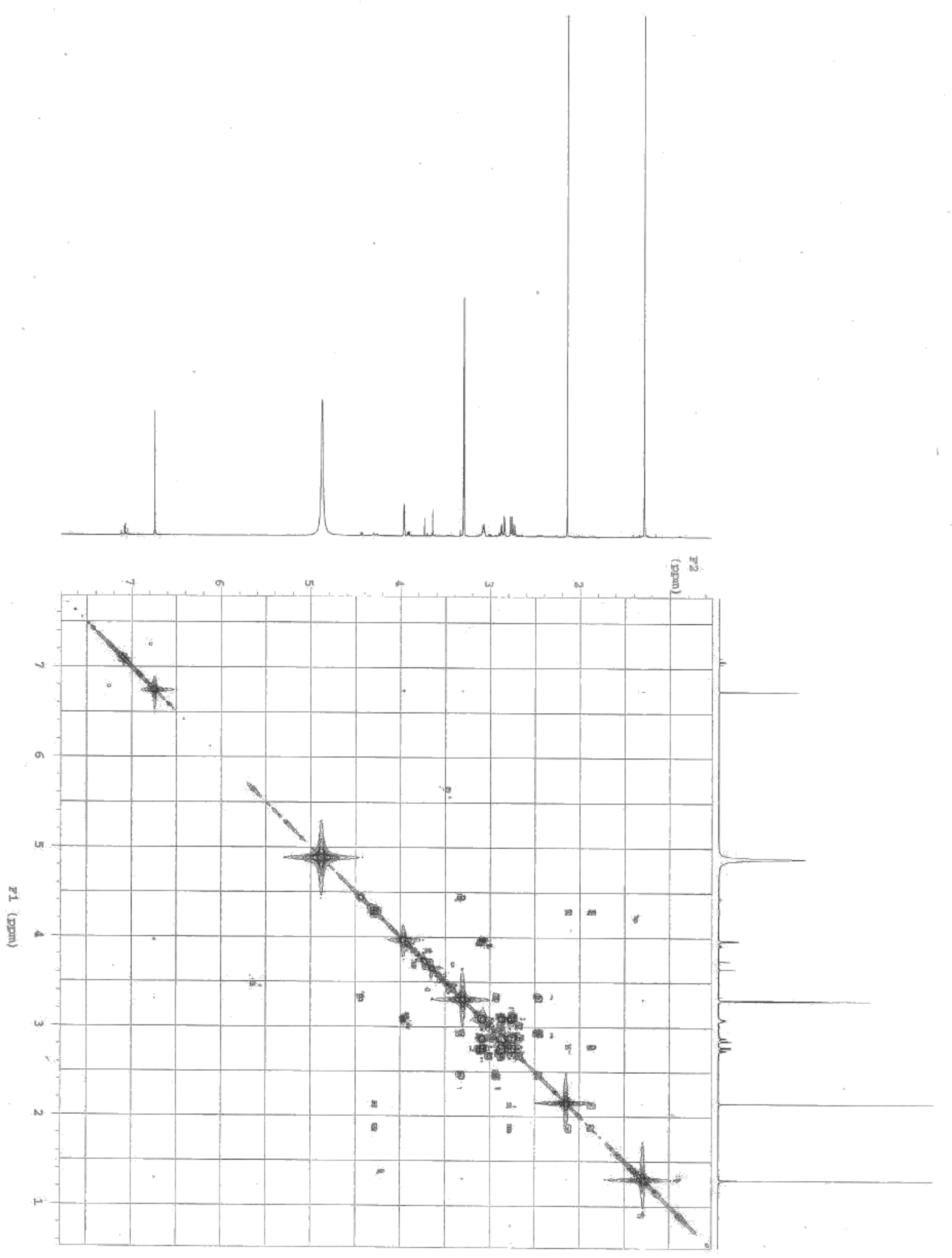


Figure S22. NOESY (500 MHz, CD 3 OD) spectrum of 2.

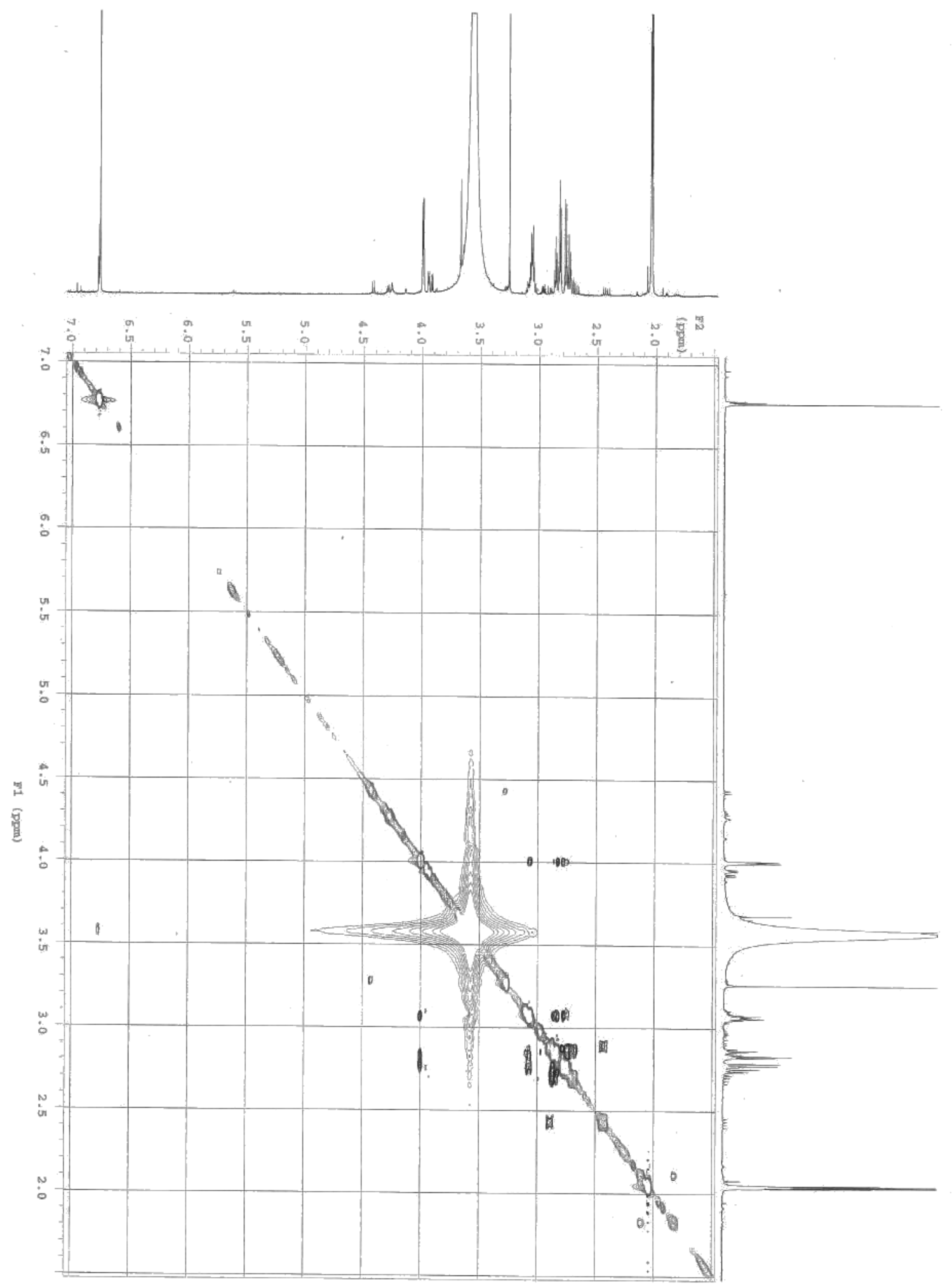


Figure S23. ${ }^{1} \mathrm{H}$ and ${ }^{13} \mathrm{C}$ NMR $\left(500 \mathrm{MHz}\right.$ for ${ }^{1} \mathrm{H}$ and $125 \mathrm{MHz}$ for ${ }^{13} \mathrm{C}$, acetone- $\left.d_{6}\right)$ spectra of 3 .
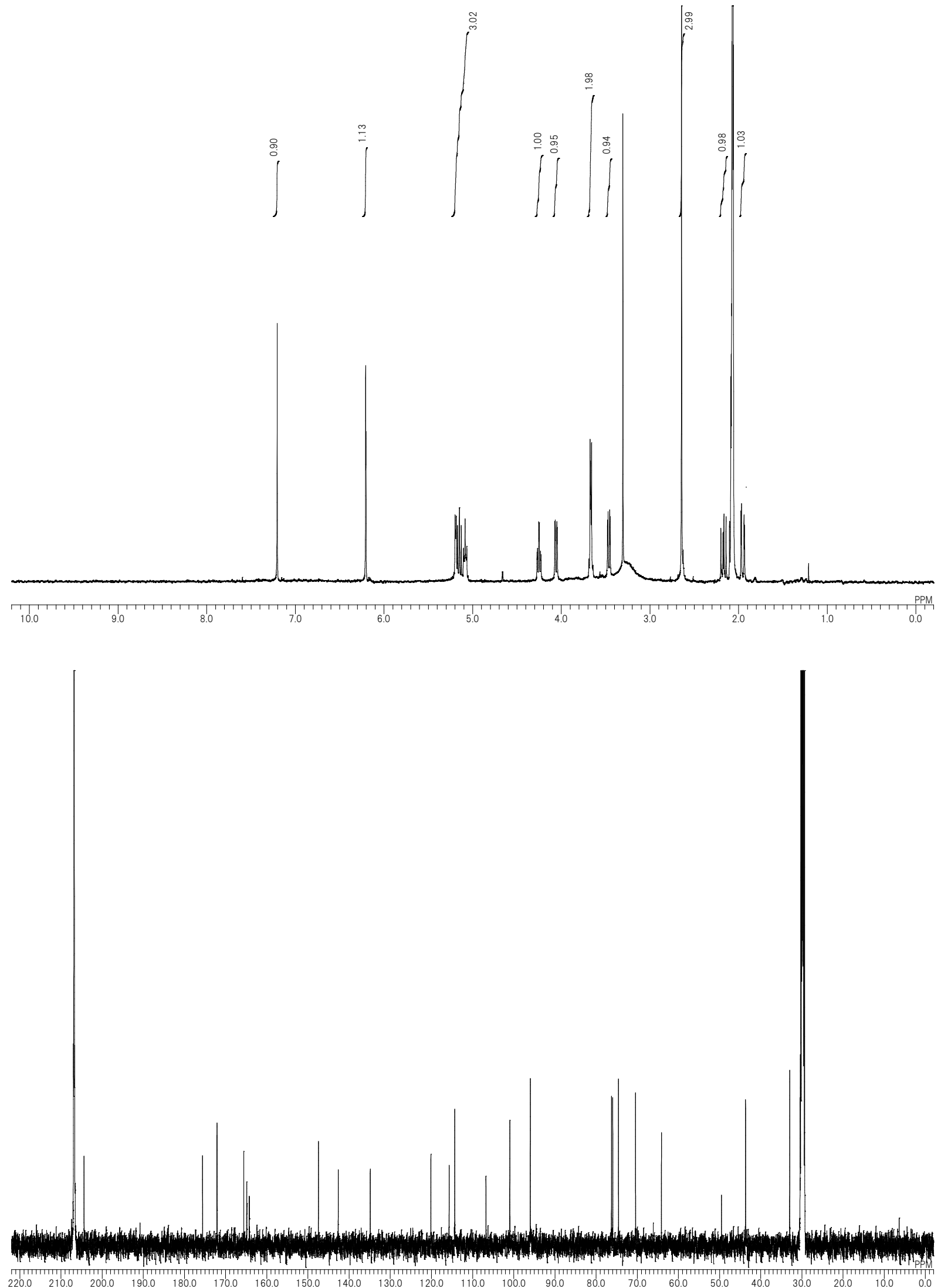
Figure S24. HMBC (500 MHz, acetone- $d_{6}$ ) spectrum of 3.

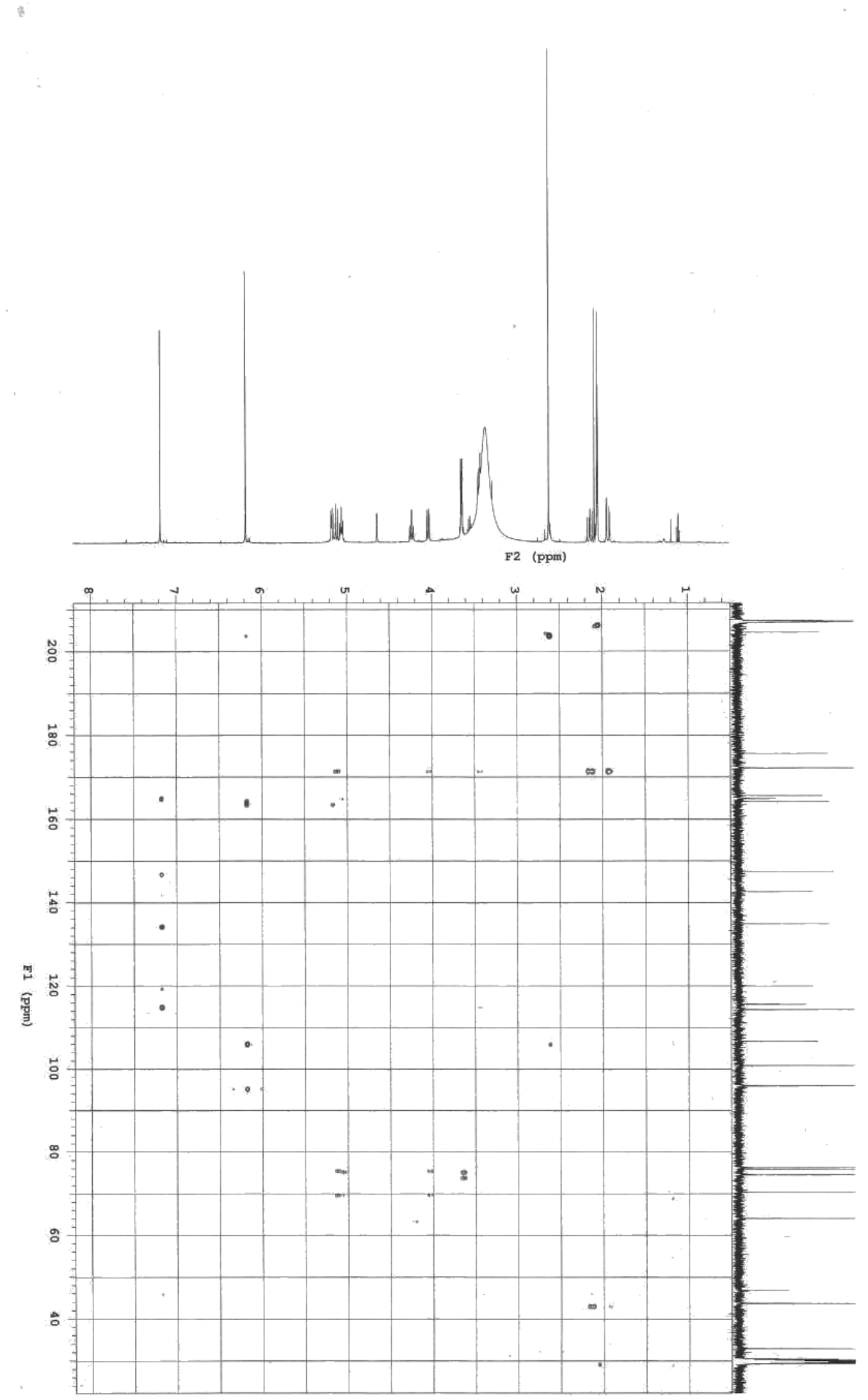


Figure S25. COSY (500 MHz, acetone- $\left.d_{6}\right)$ spectrum of 3 .

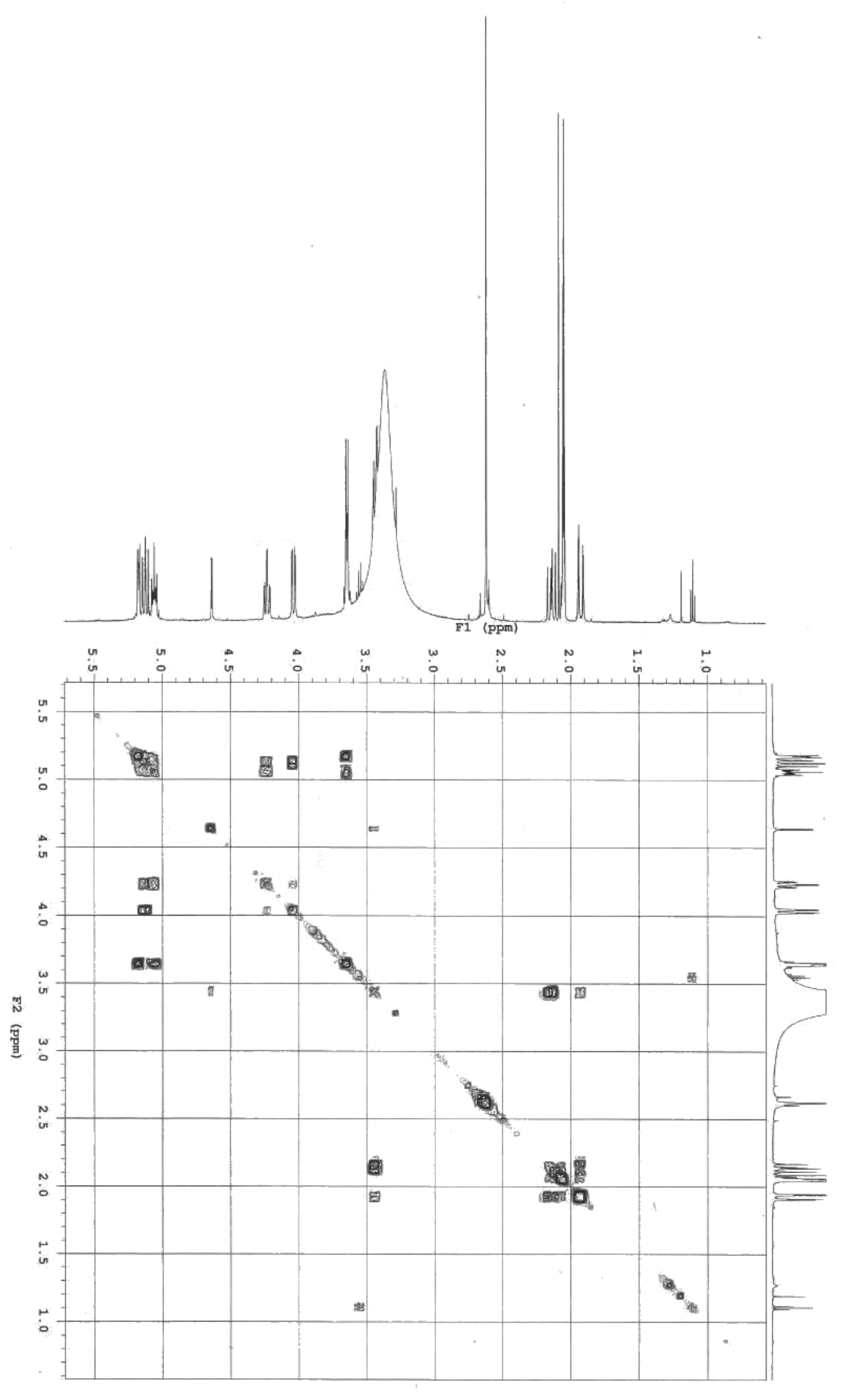


Figure S26. ${ }^{1} \mathrm{H}$ and ${ }^{13} \mathrm{C}$ NMR (400 MHz for ${ }^{1} \mathrm{H}$ and $100 \mathrm{MHz}$ for ${ }^{13} \mathrm{C}$, acetone- $d_{6}$ ) spectra of 5 .
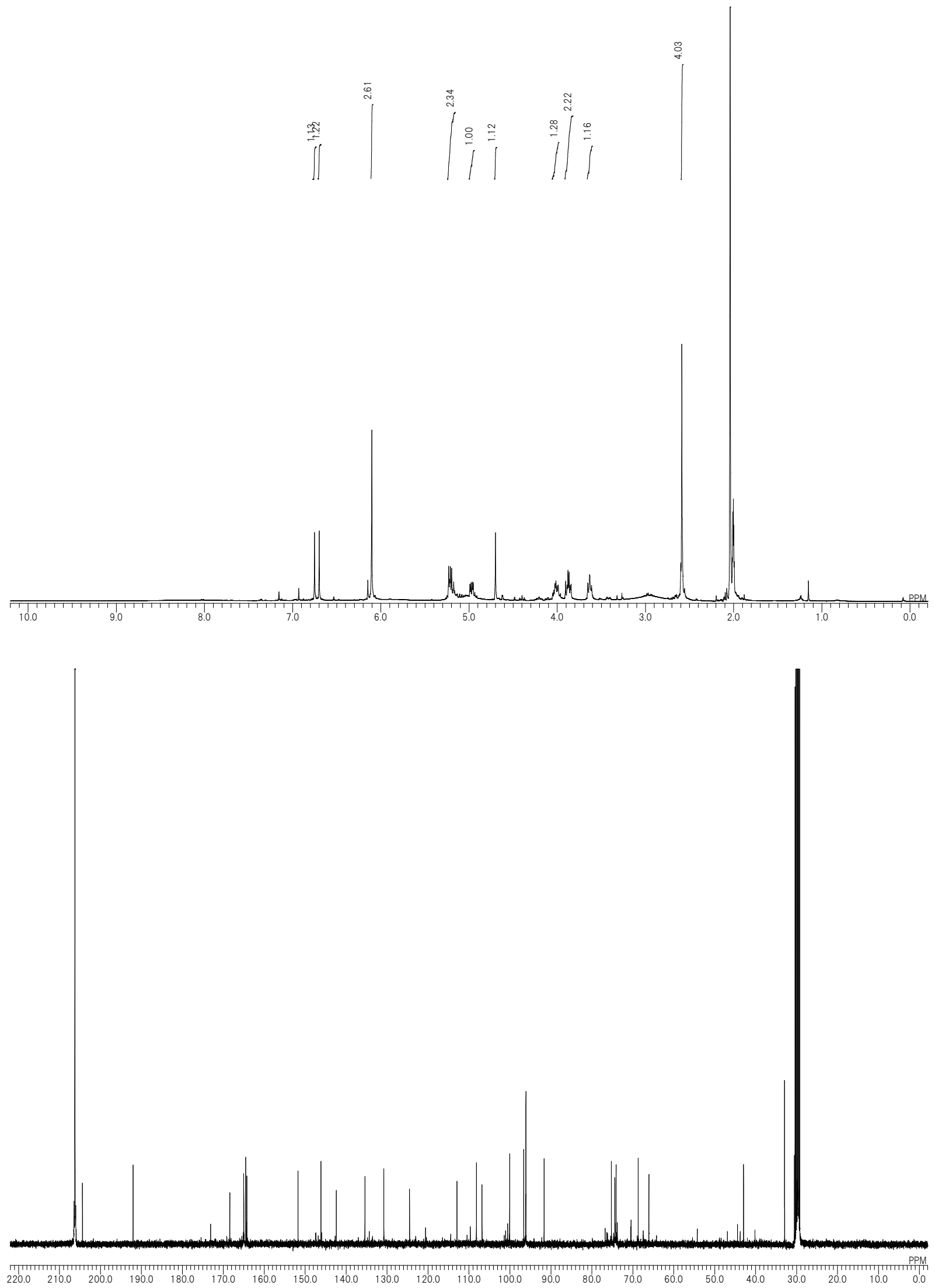
Figure S27. HMBC (500 MHz, acetone- $\left.d_{6}\right)$ spectrum of 5.

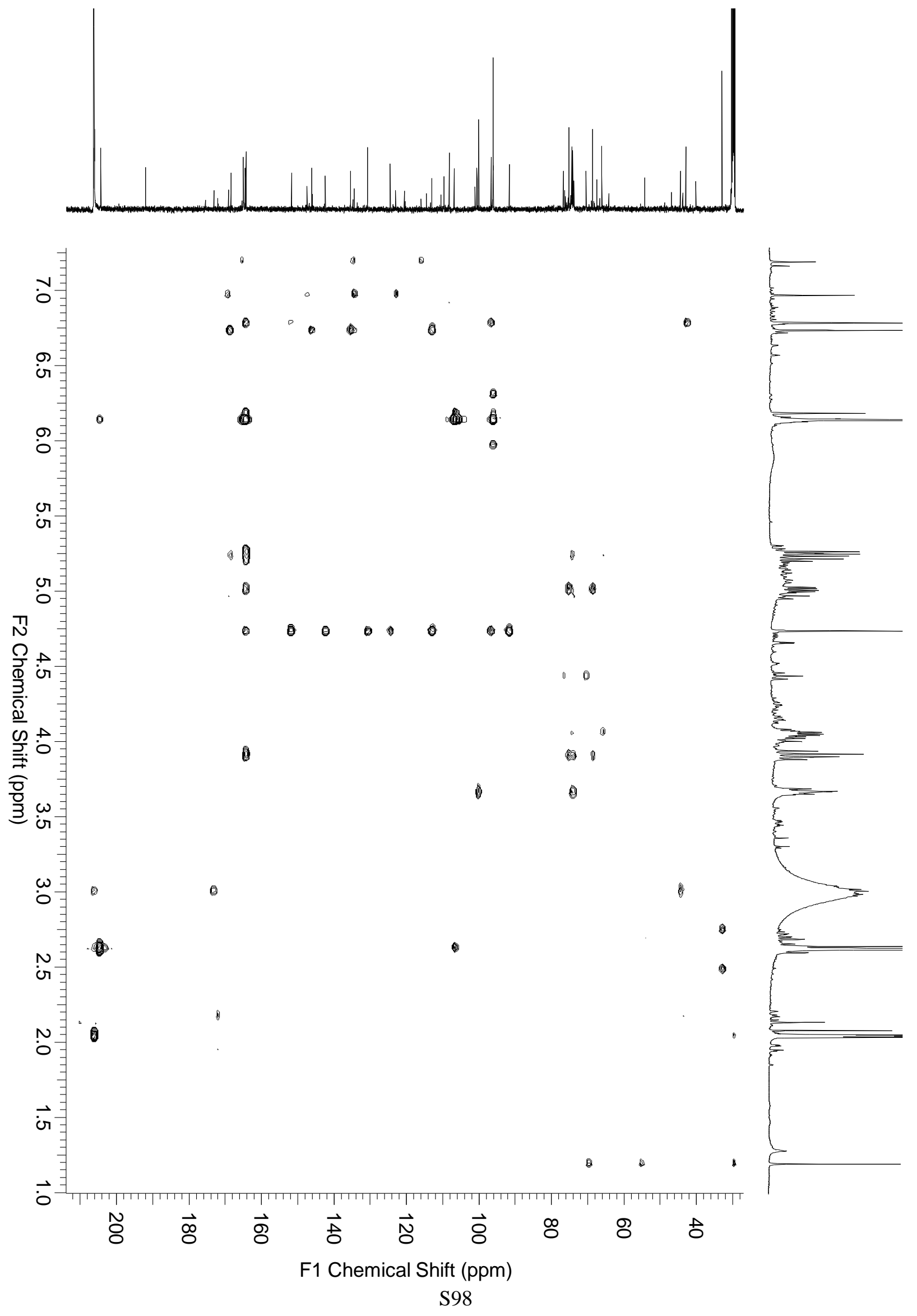


Figure S28. COSY (500 MHz, acetone- $\left.d_{6}\right)$ spectrum of 5.

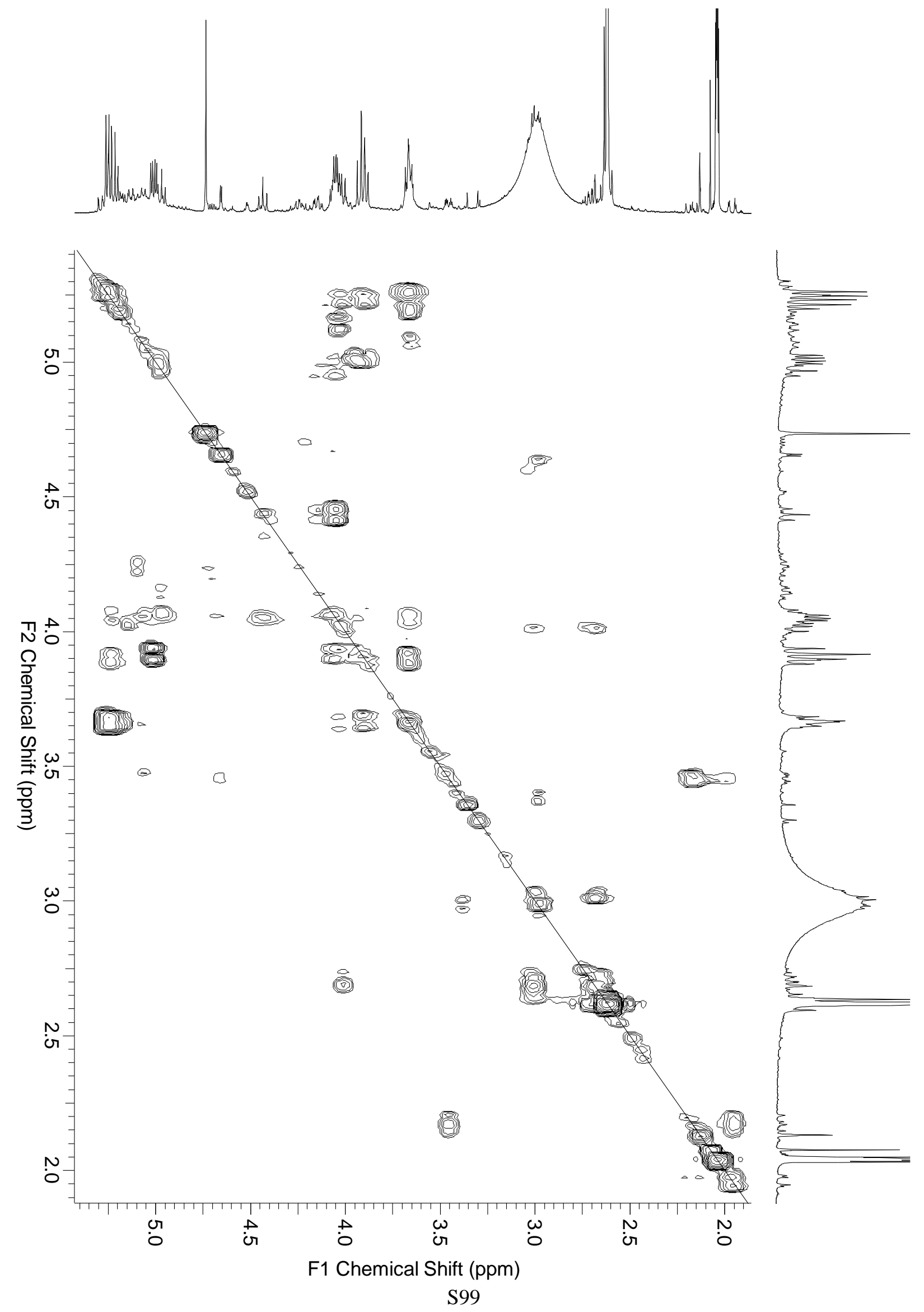


Figure S29. HPLC profiles of dried and fresh stem of Penthorum chinense.

Dried stem used for in this research $\left(5 \mathrm{~g} / 100 \mathrm{~mL} 60 \% \mathrm{CH}_{3} \mathrm{CN}\right)$ and fresh stem $(25 \mathrm{~g} / 100 \mathrm{~mL} 60 \%$ $\mathrm{CH}_{3} \mathrm{CN}$ ) were extracted at room temperature for $18 \mathrm{~h}$. The presence of the compound 5 in the fresh stem was confirmed by addition of $o$-phenylenediamine-AcOH to the extract, which yielded the phenazine derivative of $5\left(t_{R}=49.4 \mathrm{~min}\right)$.

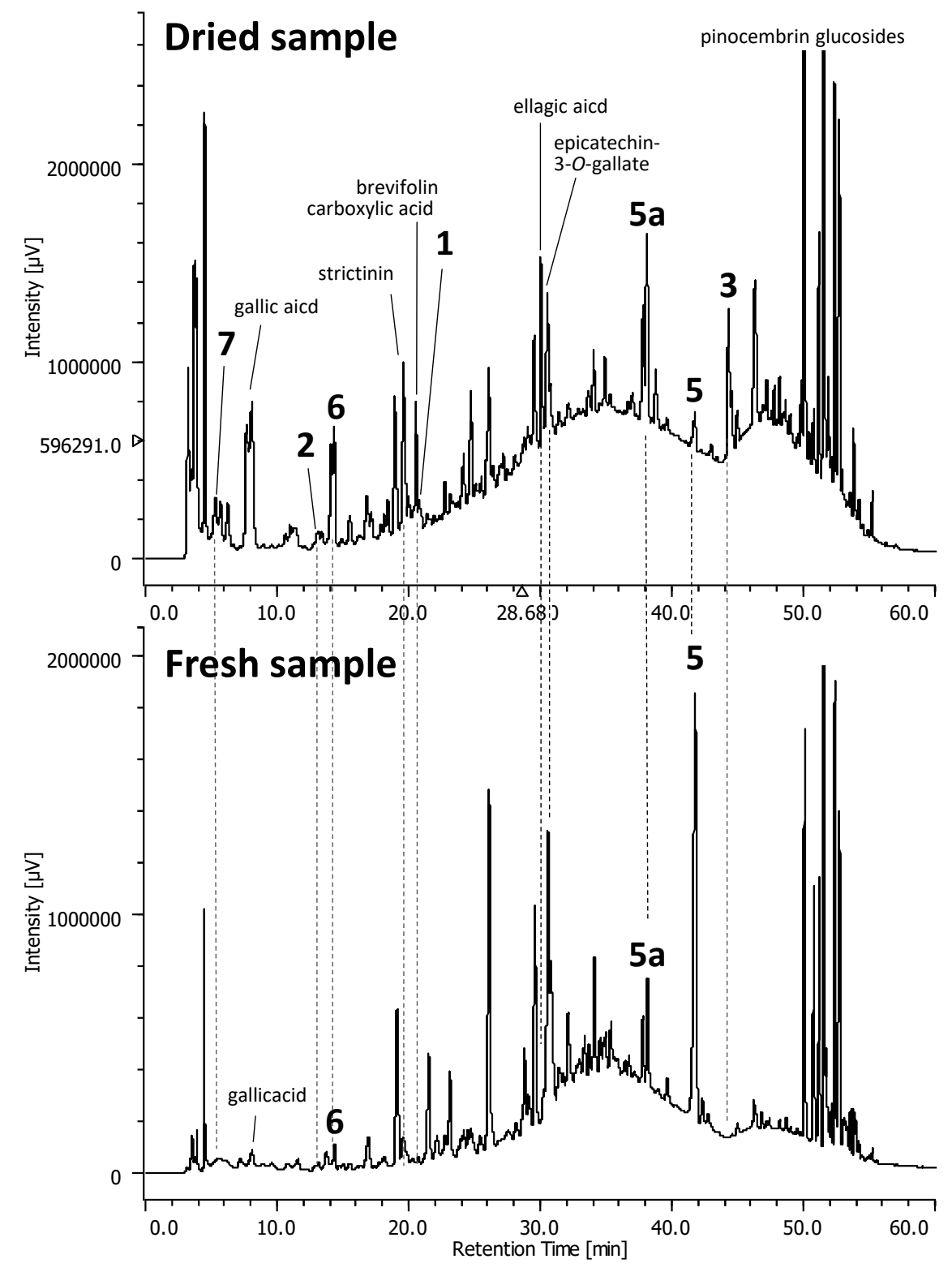


Figure S30. HPLC profiles of compound 5 (A) and extract of fresh stem (B) after treatment with $o$ phenylenediamine-AcOH.

Compound 5 in the fresh stem was further confirmed by addition of $o$-phenylenediamine-AcOH to the extract, which yielded the phenazine derivative of $\mathbf{5}\left(t_{R}=49.4 \mathrm{~min}\right)$.

A

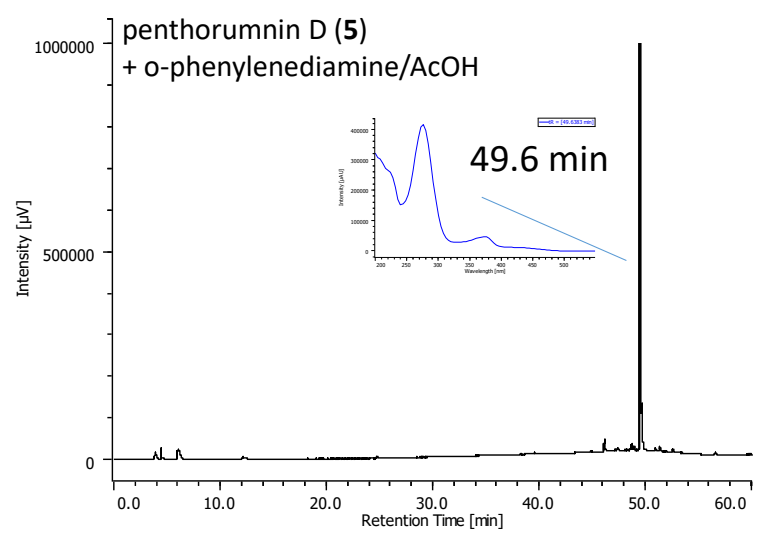

B

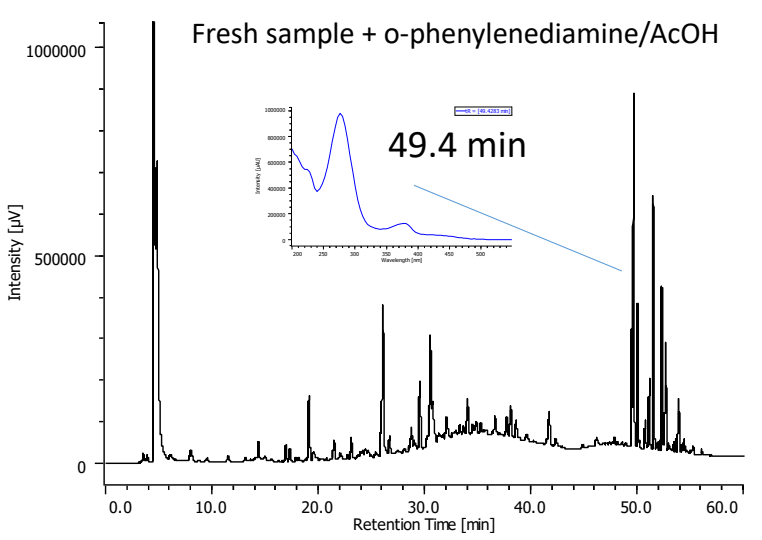

phenazine derivative of 5 
Cartesian coordinates of the optimized geometries.

Cartesian coordinates of the lowest-energy conformer of $\mathbf{1 A}(\mathbf{1 A - 1})$ at the B3LYP/6-31G(d,p) level in $\mathrm{MeOH}$ (PCM).

\begin{tabular}{|c|c|c|}
\hline 3.07199800 & -1.51560400 & 0.06739100 \\
\hline 2.17889000 & -0.66764200 & -0.64502500 \\
\hline 0.92451300 & -1.30334500 & -0.91084500 \\
\hline 0.25066300 & -1.54382200 & 0.44627300 \\
\hline 1.09627400 & -2.48681200 & 1.28899800 \\
\hline 2.49817000 & -1.86265800 & 1.44185600 \\
\hline 0.09611100 & -0.45197300 & -1.86620700 \\
\hline 0.66663800 & 0.54641900 & -1.14248500 \\
\hline 1.09165000 & -2.02321900 & 0.20044600 \\
\hline 3.37572300 & -2.76192900 & 2.09079400 \\
\hline 0.54769300 & -2.76218100 & 2.56923600 \\
\hline 4.25945500 & -0.77609200 & 0.25869100 \\
\hline-1.83596200 & 0.92539400 & -1.71366100 \\
\hline-2.20373800 & 0.53447400 & -2.80202100 \\
\hline-2.61003000 & 1.87712100 & -0.81781000 \\
\hline-4.33470100 & 2.18866400 & 0.80945100 \\
\hline-3.23331200 & 1.18097400 & 0.45335400 \\
\hline-3.90734900 & -0.13433100 & 0.10197000 \\
\hline-5.12448600 & -0.05751100 & -0.58551700 \\
\hline-5.80384800 & -1.20346200 & -1.00233900 \\
\hline-5.24594300 & -2.46613500 & -0.75942000 \\
\hline-4.01752100 & -2.56819100 & -0.10699500 \\
\hline-3.36723800 & -1.41229500 & 0.33570900 \\
\hline-2.03964900 & -1.49545700 & 1.00973800 \\
\hline-1.79268200 & -1.05896100 & 2.12103800 \\
\hline-6.99942000 & -1.15532900 & -1.65578400 \\
\hline 5.89241300 & -3.59129000 & -1.16590900 \\
\hline 5.20279700 & -0.83621400 & -0.73274600 \\
\hline 5.06029300 & -1.50757600 & -1.73728000 \\
\hline 6.38837500 & -0.00723200 & -0.42505800 \\
\hline 6.48242100 & 0.74511000 & 0.75476400 \\
\hline 7.62335000 & 1.50706500 & 0.99278900 \\
\hline 8.66578100 & 1.51669000 & 0.05611300 \\
\hline 8.56522800 & 0.76214900 & -1.12173100 \\
\hline 7.43042700 & -0.00012600 & -1.36730800 \\
\hline 9.64914100 & 0.85858000 & -1.95082000 \\
\hline 9.75883200 & 2.27912100 & 0.33816300 \\
\hline 7.72426600 & 2.24235900 & 2.13592600 \\
\hline-5.00809500 & 2.35842900 & -0.57779700 \\
\hline-3.82014100 & 2.54211900 & -1.53427900 \\
\hline-5.73670400 & 1.13530300 & -0.89209000 \\
\hline-3.73826800 & 3.41604900 & 1.20281100 \\
\hline-5.91509300 & 3.39557500 & -0.64017700 \\
\hline 3.28663400 & -2.41925500 & -0.51541300 \\
\hline 1.10405100 & -2.26343400 & -1.41805900 \\
\hline 0.17237100 & -0.57939900 & 0.95342500 \\
\hline 1.20014300 & -3.45176300 & 0.77557700 \\
\hline 2.39957300 & -0.92318300 & 2.00799200 \\
\hline 0.59833600 & -1.08517600 & -2.41701400 \\
\hline 0.75795600 & 0.04907000 & -2.57716800 \\
\hline 2.90876500 & -3.05594000 & 2.88822200 \\
\hline .05395100 & -2.03453200 & 2.80202000 \\
\hline-1.91169300 & 2.63763700 & -0.46158600 \\
\hline-2.50867600 & 1.06421600 & 1.25634600 \\
\hline-3.59033700 & -3.55030200 & 0.05963800 \\
\hline .7 .24368600 & -0.22615700 & -1.79113700 \\
\hline 6.71307400 & -3.32704600 & -1.61136600 \\
\hline 5.68361700 & 0.74503200 & 1.48481800 \\
\hline 7.33797300 & -0.58929700 & -2.27297800 \\
\hline 9.52107900 & 0.31495700 & -2.74026800 \\
\hline 10.39496500 & 2.19057700 & -0.38922200 \\
\hline 8.57846000 & 2.70211000 & 2.12639600 \\
\hline 4.03176100 & 2.06828200 & -2.49341500 \\
\hline 3.64486800 & 3.60533400 & -1.70255900 \\
\hline 4.36766600 & 4.12427700 & 0.98728500 \\
\hline 6.56861700 & 3.22365400 & 0.06589200 \\
\hline 33931100 & 1.81378400 & 1.90148200 \\
\hline 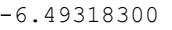 & 2.21093200 & 1.88664100 \\
\hline 4550 & 1.10657900 & 2.89931900 \\
\hline-5.49246700 & 7697 & 3.5817310 \\
\hline
\end{tabular}


Cartesian coordinates of the lowest-energy conformer of 1B (1B-1) at the B3LYP/6-31G(d,p) level in $\mathrm{MeOH}(\mathrm{PCM})$.

\begin{tabular}{|c|c|c|c|}
\hline C & 3.00427800 & -1.54901700 & 0.20817700 \\
\hline o & 2.08903000 & -0.77911900 & -0.56246400 \\
\hline $\mathrm{C}$ & 0.83945400 & -1.45072500 & -0.74968500 \\
\hline C & 0.19031300 & -1.57815800 & 0.63447700 \\
\hline C & 1.06258400 & -2.42605900 & 1.54774100 \\
\hline $\mathrm{C}$ & 2.45814600 & -1.77427300 & 1.61901100 \\
\hline C & -0.01590600 & -0.69534600 & -1.76165000 \\
\hline O & -0.78841500 & 0.34542400 & -1.11107800 \\
\hline o & -1.14467000 & -2.10350800 & 0.45260800 \\
\hline 0 & 3.35767100 & -2.60112800 & 2.33115000 \\
\hline 0 & 0.53475100 & -2.58923400 & 2.85563400 \\
\hline 0 & 4.18677700 & -0.78391300 & 0.30848600 \\
\hline $\mathrm{C}$ & -1.95789800 & 0.67781100 & -1.70962000 \\
\hline 0 & -2.32229800 & 0.21181400 & -2.76875100 \\
\hline $\mathrm{C}$ & -2.73292600 & 1.69276900 & -0.88449000 \\
\hline $\mathrm{C}$ & -4.49273100 & 2.08778300 & 0.74763000 \\
\hline C & -3.33468700 & 1.09315700 & 0.43972900 \\
\hline $\mathrm{C}$ & -3.98427200 & -0.26036600 & 0.19694200 \\
\hline C & -5.19337900 & -0.25526700 & -0.51223700 \\
\hline C & -5.84701100 & -1.44140500 & -0.85115300 \\
\hline C & -5.26788200 & -2.67163700 & -0.51044400 \\
\hline $\mathrm{C}$ & -4.04660600 & -2.70351500 & 0.16425500 \\
\hline C & -3.42340900 & -1.50843400 & 0.53183400 \\
\hline C & -2.09623100 & -1.51361600 & 1.21173000 \\
\hline 0 & -1.85496300 & -0.96843000 & 2.27502500 \\
\hline 0 & -7.03480400 & -1.46487100 & -1.51783800 \\
\hline O & -5.88688700 & -3.83448200 & -0.84077700 \\
\hline $\mathrm{C}$ & 5.11844200 & -0.93754800 & -0.68423700 \\
\hline 0 & 4.96603200 & -1.70374600 & -1.61684800 \\
\hline $\mathrm{C}$ & 6.30478000 & -0.08026700 & -0.47269700 \\
\hline $\mathrm{C}$ & 6.41154800 & 0.78071000 & 0.62918300 \\
\hline C & 7.55376900 & 1.56279200 & 0.77955300 \\
\hline C & 8.58438000 & 1.48513300 & -0.16708700 \\
\hline $\mathrm{C}$ & 8.47091600 & 0.62225700 & -1.26675800 \\
\hline $\mathrm{C}$ & 7.33490600 & -0.16100300 & -1.42443700 \\
\hline 0 & 9.54357300 & 0.63976500 & -2.11553100 \\
\hline 0 & 9.67911000 & 2.27188400 & 0.02844300 \\
\hline O & 7.66754200 & 2.40248400 & 1.84708300 \\
\hline $\mathrm{C}$ & -5.13150400 & 2.16994900 & -0.67456300 \\
\hline C & -3.94875800 & 2.29978300 & -1.63811700 \\
\hline 0 & -5.82394600 & 0.89987700 & -0.89634500 \\
\hline 0 & -5.43160400 & 1.65643200 & 1.72030000 \\
\hline 0 & -6.07685400 & 3.15339800 & -0.85504100 \\
\hline $\mathrm{H}$ & 3.21965500 & -2.50011700 & -0.29325000 \\
\hline $\mathrm{H}$ & 1.02256400 & -2.44997600 & -1.17311400 \\
\hline $\mathrm{H}$ & 0.09956600 & -0.57299300 & 1.05253900 \\
\hline $\mathrm{H}$ & 1.17246000 & -3.43314900 & 1.12494100 \\
\hline $\mathrm{H}$ & 2.35807000 & -0.78788700 & 2.09840700 \\
\hline $\mathrm{H}$ & -0.70509300 & -1.38298100 & -2.25052000 \\
\hline $\mathrm{H}$ & 0.62848700 & -0.24278200 & -2.51953900 \\
\hline $\mathrm{H}$ & 2.90938500 & -2.82504400 & 3.16132800 \\
\hline $\mathrm{H}$ & -0.06194400 & -1.84323800 & 3.03589700 \\
\hline $\mathrm{H}$ & -2.03726000 & 2.48444700 & -0.60075600 \\
\hline $\mathrm{H}$ & -2.60114300 & 1.04592600 & 1.24257700 \\
\hline $\mathrm{H}$ & -3.60362200 & -3.66305300 & 0.40430100 \\
\hline $\mathrm{H}$ & -7.29343800 & -0.55389700 & -1.72924300 \\
\hline $\mathrm{H}$ & -6.70613300 & -3.62121000 & -1.31557500 \\
\hline $\mathrm{H}$ & 5.62181000 & 0.84792200 & 1.36594500 \\
\hline $\mathrm{H}$ & 7.23316000 & -0.83278500 & -2.26943400 \\
\hline $\mathrm{H}$ & 9.40425200 & 0.02542500 & -2.84946000 \\
\hline $\mathrm{H}$ & 10.30662500 & 2.11624600 & -0.69515100 \\
\hline $\mathrm{H}$ & 8.52081200 & 2.85970000 & 1.78385000 \\
\hline $\mathrm{H}$ & -4.15991200 & 1.76581500 & -2.56551900 \\
\hline $\mathrm{H}$ & -3.77814200 & 3.35218500 & -1.86296000 \\
\hline $\mathrm{H}$ & -5.34790200 & 0.70009400 & 1.85940500 \\
\hline $\mathrm{H}$ & -6.77565600 & 3.01991000 & -0.19399800 \\
\hline $\mathrm{C}$ & -3.97669500 & 3.46236400 & 1.23204500 \\
\hline 0 & -3.26435600 & 4.19198000 & 0.57485100 \\
\hline 0 & -4.36976000 & 3.78907300 & 2.46519200 \\
\hline $\mathrm{H}$ & -4.93577200 & 3.05462900 & 2.78574500 \\
\hline
\end{tabular}


Cartesian coordinates of the lowest-energy conformer of $\mathbf{1 C}(\mathbf{1 C}-1)$ at the B3LYP/6-31G(d,p) level in $\mathrm{MeOH}(\mathrm{PCM})$.

\begin{tabular}{|c|c|c|c|}
\hline C & 3.03381200 & 0.00228800 & 0.85637300 \\
\hline 0 & 2.35147300 & -0.24341700 & -0.36708000 \\
\hline $\mathrm{C}$ & 0.95721500 & -0.48059600 & -0.17784300 \\
\hline C & 0.33402900 & 0.82979000 & 0.33804100 \\
\hline C & 0.95753300 & 1.14631300 & 1.69906300 \\
\hline $\mathrm{C}$ & 2.48513000 & 1.26506300 & 1.51510900 \\
\hline C & 0.38183300 & -0.98980300 & -1.50084400 \\
\hline O & -0.78726100 & -1.75048200 & -1.15092400 \\
\hline 0 & -1.10990200 & 0.67340700 & 0.38166700 \\
\hline O & 3.11606000 & 1.44440100 & 2.76738000 \\
\hline 0 & 0.48003600 & 2.32060100 & 2.33349600 \\
\hline 0 & 4.38809400 & 0.22146100 & 0.52297400 \\
\hline C & -1.99512200 & -1.39192500 & -1.64437500 \\
\hline 0 & -2.16171600 & -0.67785600 & -2.61029300 \\
\hline $\mathrm{C}$ & -3.06732900 & -1.97081400 & -0.75378800 \\
\hline C & -4.73003900 & -1.70746800 & 0.90568300 \\
\hline $\mathrm{C}$ & -3.49835100 & -0.95550200 & 0.37045500 \\
\hline C & -3.96534600 & 0.38969900 & -0.18192300 \\
\hline C & -5.26081400 & 0.42277200 & -0.71744600 \\
\hline C & -5.85956000 & 1.59607300 & -1.18687100 \\
\hline $\mathrm{C}$ & -5.15642500 & 2.80258800 & -1.12345900 \\
\hline C & -3.86445900 & 2.79991400 & -0.61804400 \\
\hline C & -3.25350400 & 1.61661100 & -0.16406600 \\
\hline $\mathrm{C}$ & -1.83335000 & 1.80891800 & 0.22686600 \\
\hline 0 & -1.31917000 & 2.91658000 & 0.33657800 \\
\hline 0 & -7.11646200 & 1.61560800 & -1.69917400 \\
\hline O & -5.71808100 & 3.96005800 & -1.56439000 \\
\hline C & 5.19604100 & -0.88175100 & 0.43345300 \\
\hline 0 & 4.79642100 & -2.00947200 & 0.65377700 \\
\hline $\mathrm{C}$ & 6.58080400 & -0.52986800 & 0.05196200 \\
\hline $\mathrm{C}$ & 6.97128300 & 0.79583200 & -0.18675900 \\
\hline C & 8.28958500 & 1.06837300 & -0.54277700 \\
\hline $\mathrm{C}$ & 9.21351300 & 0.02095200 & -0.65925900 \\
\hline $\mathrm{C}$ & 8.81624400 & -1.30240900 & -0.41940000 \\
\hline $\mathrm{C}$ & 7.50309300 & -1.58318700 & -0.06468800 \\
\hline O & 9.81173800 & -2.22900000 & -0.56701200 \\
\hline 0 & 10.48989700 & 0.34351900 & -1.00935200 \\
\hline 0 & 8.67965200 & 2.35314400 & -0.77676700 \\
\hline $\mathrm{C}$ & -5.49396100 & -1.94996500 & -0.42387200 \\
\hline $\mathrm{C}$ & -4.40266200 & -2.37376100 & -1.42851800 \\
\hline 0 & -6.07568000 & -0.68230300 & -0.81925600 \\
\hline 0 & -4.32084000 & -2.94561600 & 1.46831600 \\
\hline 0 & -6.52488900 & -2.86079600 & -0.32174700 \\
\hline $\mathrm{H}$ & 2.94988400 & -0.86571400 & 1.52067500 \\
\hline $\mathrm{H}$ & 0.80817400 & -1.27652100 & 0.56640800 \\
\hline $\mathrm{H}$ & 0.56031900 & 1.62806300 & -0.37498300 \\
\hline $\mathrm{H}$ & 0.75859100 & 0.30822700 & 2.38171300 \\
\hline $\mathrm{H}$ & 2.68861800 & 2.11156000 & 0.84025600 \\
\hline $\mathrm{H}$ & 1.10296800 & -1.66353900 & -1.96856900 \\
\hline $\mathrm{H}$ & 0.12071300 & -0.19181400 & -2.19838200 \\
\hline $\mathrm{H}$ & 2.61966500 & 2.15249700 & 3.20735800 \\
\hline $\mathrm{H}$ & 0.03277100 & 2.86200100 & 1.65748100 \\
\hline $\mathrm{H}$ & -2.64040200 & -2.83139500 & -0.24048800 \\
\hline $\mathrm{H}$ & -2.72512900 & -0.83475700 & 1.12484600 \\
\hline $\mathrm{H}$ & -3.30593000 & 3.72635500 & -0.5930550 \\
\hline $\mathrm{H}$ & -7.47015400 & 0.71120100 & -1.6920640 \\
\hline $\mathrm{H}$ & -6.61129300 & 3.76364500 & -1.88805700 \\
\hline $\mathrm{H}$ & 6.26617200 & 1.61202700 & -0.09888100 \\
\hline $\mathrm{H}$ & 7.18103500 & -2.60154700 & 0.12319500 \\
\hline $\mathrm{H}$ & 9.47848900 & -3.11721600 & -0.37903600 \\
\hline $\mathrm{H}$ & 11.01626900 & -0.47076700 & -1.04828300 \\
\hline $\mathrm{H}$ & 9.62135100 & 2.34621100 & -1.00969800 \\
\hline $\mathrm{H}$ & -4.45166400 & -3.44814400 & -1.61126400 \\
\hline $\mathrm{H}$ & -4.55419000 & -1.85438700 & -2.3769290 \\
\hline $\mathrm{H}$ & -5.05447900 & -3.57144100 & 1.348197 \\
\hline $\mathrm{H}$ & -7.11212900 & -2.52367700 & 0.38305100 \\
\hline $\mathrm{C}$ & -5.61113100 & -1.03161400 & 1.95849600 \\
\hline 0 & -6.79996200 & -1.28427100 & 2.06531000 \\
\hline 0 & -4.94047900 & -0.23521200 & 2.79204800 \\
\hline $\mathrm{H}$ & -5.55943200 & 0.08717700 & 3.472702 \\
\hline
\end{tabular}


Cartesian coordinates of the lowest-energy conformer of 1D (1D-1) at the B3LYP/6-31G(d,p) level in $\mathrm{MeOH}(\mathrm{PCM})$.

\begin{tabular}{|c|c|c|c|}
\hline C & 2.98352800 & -0.06170200 & 0.85126800 \\
\hline 0 & 2.28814400 & -0.10890000 & -0.38957500 \\
\hline C & 0.89038700 & -0.34370900 & -0.22507100 \\
\hline $\mathrm{C}$ & 0.29655300 & 0.88166400 & 0.49521500 \\
\hline C & 0.93879800 & 0.97660300 & 1.87968400 \\
\hline $\mathrm{C}$ & 2.46564400 & 1.09993800 & 1.69412600 \\
\hline C & 0.29248600 & -0.62509400 & -1.60478300 \\
\hline O & -0.89033300 & -1.40653200 & -1.36424400 \\
\hline O & -1.14965100 & 0.73970100 & 0.52969100 \\
\hline 0 & 3.11364000 & 1.08626700 & 2.95042400 \\
\hline 0 & 0.48804100 & 2.04750700 & 2.69166600 \\
\hline 0 & 4.33975300 & 0.17456800 & 0.53969600 \\
\hline $\mathrm{C}$ & -2.09584400 & -0.94612700 & -1.77103700 \\
\hline 0 & -2.25890300 & -0.08483800 & -2.60860400 \\
\hline $\mathrm{C}$ & -3.16657000 & -1.64663800 & -0.96788200 \\
\hline C & -4.83955000 & -1.62876800 & 0.76683900 \\
\hline C & -3.57226200 & -0.83535300 & 0.31192800 \\
\hline C & -4.01822300 & 0.58522200 & -0.03432200 \\
\hline C & -5.31147000 & 0.71990700 & -0.55579800 \\
\hline $\mathrm{C}$ & -5.88399300 & 1.95275700 & -0.87600800 \\
\hline $\mathrm{C}$ & -5.15028100 & 3.12550000 & -0.66812700 \\
\hline C & -3.86272400 & 3.02834100 & -0.16287400 \\
\hline C & -3.27982200 & 1.78303500 & 0.13945200 \\
\hline C & -1.85807300 & 1.89457700 & 0.55577000 \\
\hline O & -1.33028100 & 2.96477500 & 0.83687700 \\
\hline 0 & -7.14256200 & 2.06839400 & -1.37139800 \\
\hline O & -5.68183000 & 4.34170100 & -0.96413700 \\
\hline $\mathrm{C}$ & 5.12780500 & -0.92305200 & 0.30523700 \\
\hline 0 & 4.71002300 & -2.06209100 & 0.38965700 \\
\hline C & 6.51434500 & -0.54864400 & -0.04692100 \\
\hline $\mathrm{C}$ & 7.41995300 & -1.58977700 & -0.29638400 \\
\hline C & 8.73621600 & -1.29410200 & -0.63490100 \\
\hline C & 9.14686300 & 0.04330400 & -0.72549600 \\
\hline C & 8.23739700 & 1.08052900 & -0.47561600 \\
\hline $\mathrm{C}$ & 6.92007600 & 0.79390400 & -0.13509300 \\
\hline 0 & 8.76492500 & 2.33689900 & -0.59841700 \\
\hline O & 10.44500400 & 0.28209100 & -1.06195700 \\
\hline 0 & 9.62225400 & -2.30026700 & -0.87889100 \\
\hline C & -5.59156500 & -1.67547700 & -0.59607700 \\
\hline $\mathrm{C}$ & -4.51548100 & -1.90918500 & -1.67821200 \\
\hline 0 & -6.17030500 & -0.34743700 & -0.74133800 \\
\hline 0 & -5.57578800 & -0.95185600 & 1.77588000 \\
\hline 0 & -6.64814800 & -2.55691400 & -0.54865500 \\
\hline $\mathrm{H}$ & 2.88436100 & -1.01655000 & 1.38041200 \\
\hline $\mathrm{H}$ & 0.72952300 & -1.24287500 & 0.38747500 \\
\hline $\mathrm{H}$ & 0.52759700 & 1.77768200 & -0.08867600 \\
\hline $\mathrm{H}$ & 0.73417500 & 0.04688700 & 2.42905800 \\
\hline $\mathrm{H}$ & 2.67572700 & 2.03225100 & 1.14658400 \\
\hline $\mathrm{H}$ & 0.99317800 & -1.23116100 & -2.18294000 \\
\hline $\mathrm{H}$ & 0.04311500 & 0.27983900 & -2.16201400 \\
\hline $\mathrm{H}$ & 2.62967700 & 1.72597800 & 3.49639700 \\
\hline $\mathrm{H}$ & 0.03197100 & 2.68688900 & 2.11415400 \\
\hline $\mathrm{H}$ & -2.75060700 & -2.59187400 & -0.62684900 \\
\hline $\mathrm{H}$ & -2.78764000 & -0.83513400 & 1.06515000 \\
\hline $\mathrm{H}$ & -3.28308800 & 3.93095500 & -0.02023300 \\
\hline $\mathrm{H}$ & -7.52227400 & 1.18097800 & -1.47597200 \\
\hline $\mathrm{H}$ & -6.57915500 & 4.20918400 & -1.30821700 \\
\hline $\mathrm{H}$ & 7.09801300 & -2.62151700 & -0.22657200 \\
\hline $\mathrm{H}$ & 6.21781000 & 1.59593200 & 0.05865000 \\
\hline $\mathrm{H}$ & 8.08835600 & 3.00689500 & -0.42950900 \\
\hline $\mathrm{H}$ & 10.58630900 & 1.24166500 & -1.09192800 \\
\hline $\mathrm{H}$ & 10.48008400 & -1.90290200 & -1.09652600 \\
\hline $\mathrm{H}$ & -4.57258900 & -2.93621300 & -2.04222800 \\
\hline $\mathrm{H}$ & -4.67603500 & -1.22928100 & -2.51816900 \\
\hline $\mathrm{H}$ & -6.43277300 & -0.68052100 & 1.40914300 \\
\hline $\mathrm{H}$ & -7.04090600 & -2.63631600 & -1.43132800 \\
\hline $\mathrm{C}$ & -4.53920700 & -3.05106500 & 1.30735300 \\
\hline 0 & -3.92018000 & -3.90365600 & 0.70465900 \\
\hline 0 & -5.03914600 & -3.27681400 & 2.52287600 \\
\hline $\mathrm{H}$ & -5.50198800 & -2.45107600 & 2.78682700 \\
\hline
\end{tabular}


Cartesian coordinates of the lowest-energy conformer of $\mathbf{1 E}(\mathbf{1 E - 1})$ at the B3LYP/6-31G(d,p) level in $\mathrm{MeOH}(\mathrm{PCM})$.

\begin{tabular}{|c|c|c|c|}
\hline $\mathrm{C}$ & -2.92276500 & 0.83255700 & -0.10812800 \\
\hline 0 & -2.26608100 & -0.24695500 & 0.53306700 \\
\hline $\mathrm{C}$ & -0.85882600 & -0.26956000 & 0.32591300 \\
\hline C & -0.18398300 & 1.01457300 & 0.89869600 \\
\hline C & -0.98287100 & 2.28490200 & 0.51680300 \\
\hline $\mathrm{C}$ & -2.50637000 & 2.10489600 & 0.60206100 \\
\hline C & -0.43617900 & -1.59603300 & 0.97746600 \\
\hline O & 0.98363500 & -1.71900800 & 0.90763000 \\
\hline 0 & 1.15937400 & 1.01552400 & 0.29684700 \\
\hline O & -3.15058100 & 3.21982700 & $0.014941 \mathrm{C}$ \\
\hline 0 & -0.73062900 & 3.39512300 & 1.3575020 \\
\hline 0 & -4.30806300 & 0.63805400 & 0.08129900 \\
\hline C & 1.55724500 & -2.43534900 & -0.09334200 \\
\hline 0 & 1.06849100 & -3.42408000 & -0.59282100 \\
\hline $\mathrm{C}$ & 2.80472800 & -1.72434500 & -0.57419600 \\
\hline $\mathrm{C}$ & 4.68417300 & -1.67510000 & 0.92752100 \\
\hline $\mathrm{C}$ & 3.48833800 & -0.81920800 & 0.49865500 \\
\hline $\mathrm{C}$ & 3.99313300 & 0.42650700 & -0.18311600 \\
\hline C & 5.14944000 & 0.27123800 & -0.95175500 \\
\hline C & 5.74575200 & 1.35670100 & -1.59758300 \\
\hline $\mathrm{C}$ & 5.17027600 & 2.63046400 & -1.47681000 \\
\hline C & 4.00134700 & 2.79662300 & -0.73831700 \\
\hline C & 3.39192500 & 1.69526400 & -0.11664200 \\
\hline C & 2.08759400 & 1.95612700 & 0.56159700 \\
\hline 0 & 1.88463800 & 2.96618200 & 1.22400700 \\
\hline 0 & 6.87621200 & 1.24046300 & -2.34644500 \\
\hline O & 5.74164800 & 3.70204700 & -2.09263000 \\
\hline C & -4.95994600 & -0.13872800 & -0.8397330 \\
\hline 0 & -4.39664100 & -0.63621400 & -1.7965450 \\
\hline $\mathrm{C}$ & -6.39917800 & -0.28118300 & -0.53127200 \\
\hline C & -6.98179400 & 0.32533800 & 0.59109900 \\
\hline C & -8.34335500 & 0.15876100 & 0.83072900 \\
\hline C & -9.11924500 & -0.61040800 & -0.04717600 \\
\hline $\mathrm{C}$ & -8.53015900 & -1.21405200 & -1.16755200 \\
\hline $\mathrm{C}$ & -7.17276500 & -1.05323000 & -1.41388200 \\
\hline O & -9.39459100 & -1.93652800 & -1.94347300 \\
\hline 0 & -10.44563500 & -0.74086500 & 0.23467700 \\
\hline 0 & -8.91947200 & 0.74416900 & 1.9183280 \\
\hline $\mathrm{C}$ & 5.21031600 & -2.11799400 & -0.45770300 \\
\hline $\mathrm{C}$ & 3.93689200 & -2.60795600 & -1.16815600 \\
\hline 0 & 5.77560400 & -0.94436900 & -1.12406500 \\
\hline 0 & 4.19272300 & -2.80446500 & 1.63806300 \\
\hline 0 & 6.20064500 & -3.07538700 & -0.42253600 \\
\hline $\mathrm{H}$ & -2.69078000 & 0.84908900 & -1.17929700 \\
\hline $\mathrm{H}$ & -0.63328800 & -0.30396700 & -0.75088600 \\
\hline $\mathrm{H}$ & -0.07843200 & 0.95879600 & 1.98671900 \\
\hline $\mathrm{H}$ & -0.73971900 & 2.51455800 & -0.53416100 \\
\hline $\mathrm{H}$ & -2.78176500 & 1.99485200 & 1.66145200 \\
\hline $\mathrm{H}$ & -0.92548300 & -2.42947500 & 0.46930700 \\
\hline $\mathrm{H}$ & -0.71336100 & -1.60040000 & 2.03364800 \\
\hline $\mathrm{H}$ & -2.72506000 & 3.99971300 & 0.40352300 \\
\hline $\mathrm{H}$ & 0.24103500 & 3.50271400 & 1.39332200 \\
\hline $\mathrm{H}$ & 2.39781700 & -1.06767000 & -1.35221600 \\
\hline $\mathrm{H}$ & 2.83531600 & -0.59332400 & 1.33413400 \\
\hline $\mathrm{H}$ & 3.55232800 & 3.78052400 & -0.6741830 \\
\hline $\mathrm{H}$ & 7.16016000 & 0.31251400 & -2.34285400 \\
\hline $\mathrm{H}$ & 6.53423700 & 3.39740000 & -2.5619440 \\
\hline $\mathrm{H}$ & -6.39241500 & 0.92195200 & 1.27504400 \\
\hline $\mathrm{H}$ & -6.70352800 & -1.51433300 & -2.27598900 \\
\hline $\mathrm{H}$ & -8.92872700 & -2.32546500 & -2.69646100 \\
\hline $\mathrm{H}$ & -10.85581700 & -1.29338000 & -0.44947500 \\
\hline $\mathrm{H}$ & -9.86326700 & 0.51971300 & 1.91879400 \\
\hline $\mathrm{H}$ & 4.03746500 & -2.50265900 & -2.2491980 \\
\hline $\mathrm{H}$ & 3.77586200 & -3.66283600 & -0.9404840 \\
\hline $\mathrm{H}$ & 4.84605900 & -3.51703600 & $1.541205 \mathrm{C}$ \\
\hline $\mathrm{H}$ & 6.90523900 & -2.71223800 & 0.1491280 \\
\hline C & 5.77200500 & -1.03354300 & 1.79003100 \\
\hline 0 & 6.94494700 & -1.36260800 & 1.71548800 \\
\hline 0 & 5.30000500 & -0.16068700 & 2.6810760 \\
\hline $\mathrm{H}$ & 6.04191400 & 0.14558200 & 3.234356 \\
\hline
\end{tabular}


Cartesian coordinates of the lowest-energy conformer of 1F (1F-1) at the B3LYP/6-31G(d,p) level in $\mathrm{MeOH}(\mathrm{PCM})$.

\begin{tabular}{|c|c|c|}
\hline 2.86636200 & 8819700 & 9900 \\
\hline 2.18933500 & -0.25874700 & -0.46588100 \\
\hline 0.78110800 & -0.22457400 & -0.26582100 \\
\hline 0.13336500 & 0.98015700 & -1.01425500 \\
\hline 0.95656200 & 2.27595400 & -0.81267400 \\
\hline 2.47577400 & 2.05372800 & -0.86891700 \\
\hline 0.33510200 & -1.61898200 & -0.73435200 \\
\hline 1.08804200 & -1.70762000 & -0.67137000 \\
\hline-1.21131800 & 1.08507100 & -0.42368700 \\
\hline 3.14430700 & 3.22784700 & -0.44868700 \\
\hline 0.72617500 & 3.26011900 & -1.80315000 \\
\hline 4.24784100 & 0.64239800 & -0.13714600 \\
\hline-1.68207100 & -2.26903000 & 0.41220700 \\
\hline 1.18590300 & -3.14011500 & 1.09048600 \\
\hline 2.95864500 & -1.52246700 & 0.74261400 \\
\hline 4.84190600 & -1.54476700 & -0.82595500 \\
\hline 3.58471300 & -0.71762100 & -0.43988100 \\
\hline 4.05782900 & 0.60860600 & 0.10120300 \\
\hline-5.21526600 & 0.55557800 & 0.88514900 \\
\hline-5.77972300 & 1.71182800 & 1.42899000 \\
\hline-5.16786000 & 2.95193500 & 1.19253100 \\
\hline-4.00051400 & 3.01871100 & 0.43434800 \\
\hline-3.42552300 & 1.85081900 & -0.08838500 \\
\hline-2.11651300 & 2.01205300 & -0.79063200 \\
\hline-1.89644800 & 2.94044800 & -1.55765500 \\
\hline-6.91023800 & 1.69688600 & 2.18543000 \\
\hline 5.70347400 & 4.08988500 & 1.71044700 \\
\hline 4.88346200 & -0.00804200 & 0.88860200 \\
\hline 4.30784600 & -0.35589800 & 1.90205700 \\
\hline 6.32081700 & -0.21581900 & 0.60984200 \\
\hline 7.08077200 & -0.87367100 & 1.58760800 \\
\hline 8.43825200 & -1.09160800 & 1.37631500 \\
\hline 9.03632300 & -0.65114900 & 0.18726900 \\
\hline 8.27192600 & 0.00573600 & -0.78712800 \\
\hline 6.91428200 & 0.22679100 & -0.58455000 \\
\hline 8.97469900 & 0.38321600 & -1.89857500 \\
\hline 10.36831900 & -0.88707300 & 0.02704600 \\
\hline 9.18368500 & -1.73017600 & 2.32161200 \\
\hline-5.36022400 & -1.87723000 & 0.60449000 \\
\hline-4.11768600 & -2.34363000 & 1.36997200 \\
\hline-5.87413100 & -0.61904700 & 1.15859300 \\
\hline-5.83918 & -0.87599300 & -1.58474200 \\
\hline-6.39659800 & -2.77781100 & 0.68287000 \\
\hline 2.63488500 & 1.06149500 & 1.07483900 \\
\hline 0.55205500 & -0.10485400 & 0.80404800 \\
\hline 0.02707800 & 0.77467500 & -2.08397300 \\
\hline 0.71804200 & 2.65718900 & 0.19398000 \\
\hline 2.74654200 & 1.78559200 & -1.90103400 \\
\hline 0.80010000 & -2.38432700 & -0.11010700 \\
\hline 0.62467500 & -1.77205500 & -1.77595300 \\
\hline 2.73034100 & 3.95258900 & -0.94244400 \\
\hline-0.24137500 & 3.39916 & -1.83596800 \\
\hline 2.59459600 & -0.79006900 & 1.47278300 \\
\hline 2.89707900 & -0.59541400 & -1.27001900 \\
\hline 3.52597500 & 3.98032400 & 0.27980600 \\
\hline 7.21646200 & 0.78035800 & 2.27462000 \\
\hline 6.49672500 & 3.85266800 & 2.21640300 \\
\hline 6.61525700 & -1.21142900 & 2.50535600 \\
\hline 6.32436700 & 0.73402500 & -1.33864300 \\
\hline 8.39711900 & 0.83946600 & -2.52579300 \\
\hline 10.64551200 & -0.52934400 & -0.83137200 \\
\hline .09714100 & -1.78714600 & 1.99996300 \\
\hline .23913100 & -2.16305000 & 2.43903800 \\
\hline .97463000 & -3.41163500 & 1.20670900 \\
\hline .64626900 & 0.07292400 & -1.62737800 \\
\hline 12780300 & -2.43710900 & 0.14198400 \\
\hline 622190 & -2.81267300 & -1.61985800 \\
\hline & -3.62915100 & -1.23898400 \\
\hline 然 & -2.93990500 & -2.78869800 \\
\hline .66833100 & -2.15166800 & -2.88458200 \\
\hline
\end{tabular}


Cartesian coordinates of the lowest-energy conformer of $\mathbf{1 G}(\mathbf{1 G - 1})$ at the B3LYP/6-31G(d,p) level in $\mathrm{MeOH}(\mathrm{PCM})$.

\begin{tabular}{|c|c|c|c|}
\hline C & 2.79055100 & 0.71839000 & 0.47703700 \\
\hline 0 & 2.18248900 & 0.19385900 & -0.69186600 \\
\hline $\mathrm{C}$ & 0.76378500 & 0.09222800 & -0.61210700 \\
\hline C & 0.13126400 & 1.49747600 & -0.41418000 \\
\hline C & 0.84759700 & 2.26952800 & 0.70936000 \\
\hline $\mathrm{C}$ & 2.37672300 & 2.17336200 & 0.61132600 \\
\hline C & 0.39461200 & -0.62851600 & -1.91893200 \\
\hline O & -1.02618700 & -0.69745400 & -2.06186000 \\
\hline 0 & -1.26096700 & 1.24032800 & -0.05227100 \\
\hline O & 2.97287100 & 2.73263700 & 1.76709800 \\
\hline 0 & 0.56790900 & 3.65847000 & 0.72003000 \\
\hline 0 & 4.18549400 & 0.65960700 & 0.26742400 \\
\hline C & -1.68402300 & -1.86616700 & -1.83161700 \\
\hline 0 & -1.17074600 & -2.96143200 & -1.83527000 \\
\hline $\mathrm{C}$ & -3.11571900 & -1.49403500 & -1.51383700 \\
\hline C & -3.85801500 & -1.93213000 & 0.77554800 \\
\hline $\mathrm{C}$ & -3.14837400 & -0.85892100 & -0.05799500 \\
\hline C & -4.01893500 & 0.36998200 & -0.11857500 \\
\hline C & -5.38092200 & 0.08057600 & -0.25971500 \\
\hline C & -6.34266400 & 1.08984800 & -0.34084600 \\
\hline $\mathrm{C}$ & -5.93419800 & 2.43212500 & -0.31365100 \\
\hline C & -4.58165300 & 2.73654600 & -0.23385900 \\
\hline C & -3.60888200 & 1.72191000 & -0.14704200 \\
\hline C & -2.19851200 & 2.19776000 & -0.19403000 \\
\hline 0 & -1.90768100 & 3.37855400 & -0.36671100 \\
\hline 0 & -7.66969200 & 0.83063400 & -0.46159200 \\
\hline O & -6.85495200 & 3.43214200 & -0.38933200 \\
\hline C & 4.81572500 & -0.51189900 & 0.5988250 \\
\hline O & 4.22672900 & -1.46137800 & 1.07924100 \\
\hline $\mathrm{C}$ & 6.26512100 & -0.46576300 & 0.30863900 \\
\hline $\mathrm{C}$ & 7.01937900 & -1.61202400 & 0.5975680 \\
\hline C & 8.38665600 & -1.62147300 & 0.34193900 \\
\hline C & 9.00005600 & -0.48431500 & -0.20226400 \\
\hline $\mathrm{C}$ & 8.24150900 & 0.65936600 & -0.48852300 \\
\hline $\mathrm{C}$ & 6.87413000 & 0.67694800 & -0.23715600 \\
\hline 0 & 8.95992900 & 1.69780000 & -1.01525700 \\
\hline 0 & 10.34095800 & -0.54234700 & -0.43501900 \\
\hline 0 & 9.12665000 & -2.73167600 & 0.61923400 \\
\hline $\mathrm{C}$ & -4.96843200 & -2.34782800 & -0.20956600 \\
\hline $\mathrm{C}$ & -4.18829500 & -2.60591000 & -1.50588000 \\
\hline 0 & -5.87637500 & -1.20363300 & -0.36162900 \\
\hline 0 & -2.95785400 & -3.00849400 & 0.99186600 \\
\hline 0 & -5.73286200 & -3.42330300 & 0.18083300 \\
\hline $\mathrm{H}$ & 2.52050500 & 0.12274600 & 1.35661500 \\
\hline $\mathrm{H}$ & 0.47602000 & -0.53087000 & 0.24823100 \\
\hline $\mathrm{H}$ & 0.15336500 & 2.07581400 & -1.34309500 \\
\hline $\mathrm{H}$ & 0.54527500 & 1.80302900 & 1.66205200 \\
\hline $\mathrm{H}$ & 2.70098700 & 2.69676100 & -0.30046100 \\
\hline $\mathrm{H}$ & 0.83059200 & -1.62910300 & -1.92137100 \\
\hline $\mathrm{H}$ & 0.77883200 & -0.06147700 & -2.76989900 \\
\hline $\mathrm{H}$ & 2.56137000 & 3.60259800 & 1.88290700 \\
\hline $\mathrm{H}$ & -0.35124200 & 3.78125300 & 0.41022800 \\
\hline $\mathrm{H}$ & -3.38260300 & -0.70893300 & -2.22232400 \\
\hline $\mathrm{H}$ & -2.16487700 & -0.64888800 & 0.34083400 \\
\hline $\mathrm{H}$ & -4.27086400 & 3.77323200 & -0.25675100 \\
\hline $\mathrm{H}$ & -7.79849700 & -0.13168600 & -0.48147200 \\
\hline $\mathrm{H}$ & -7.73623400 & 3.03290700 & -0.46038500 \\
\hline $\mathrm{H}$ & 6.54157200 & -2.48843600 & 1.01795500 \\
\hline $\mathrm{H}$ & 6.28803400 & 1.56071600 & -0.45914300 \\
\hline $\mathrm{H}$ & 8.38544000 & 2.45788300 & -1.18096600 \\
\hline $\mathrm{H}$ & 10.62789900 & 0.30635500 & -0.80795800 \\
\hline $\mathrm{H}$ & 10.04778500 & -2.55093600 & 0.37445600 \\
\hline $\mathrm{H}$ & -3.72833200 & -3.59463800 & -1.4564360 \\
\hline $\mathrm{H}$ & -4.84710300 & -2.56559500 & -2.3746350 \\
\hline $\mathrm{H}$ & -3.48609000 & -3.81164800 & $1.131085 \mathrm{C}$ \\
\hline $\mathrm{H}$ & -6.07231400 & -3.20259100 & 1.07049700 \\
\hline $\mathrm{C}$ & -4.40786600 & -1.52860000 & 2.14646700 \\
\hline 0 & -5.44147300 & -1.99085400 & 2.60229100 \\
\hline 0 & -3.59765700 & -0.71242600 & 2.82156600 \\
\hline $\mathrm{H}$ & -3.97589700 & -0.56753700 & 3.708228 \\
\hline
\end{tabular}


Cartesian coordinates of the lowest-energy conformer of $\mathbf{1 H}(\mathbf{1 H - 1})$ at the B3LYP/6-31G(d,p) level in $\mathrm{MeOH}(\mathrm{PCM})$.

\begin{tabular}{|c|c|c|c|}
\hline C & -2.75094900 & 0.73379600 & -0.55413200 \\
\hline 0 & -2.10128200 & 0.26793700 & 0.61814000 \\
\hline $\mathrm{C}$ & -0.68549000 & 0.16611200 & 0.49658800 \\
\hline $\mathrm{C}$ & -0.07275400 & 1.56354400 & 0.20875400 \\
\hline C & -0.81479100 & 2.25973100 & -0.94313600 \\
\hline $\mathrm{C}$ & -2.33953600 & 2.17804200 & -0.78484300 \\
\hline C & -0.26019800 & -0.47997900 & 1.82431900 \\
\hline 0 & 1.16634400 & -0.53305500 & 1.88895400 \\
\hline 0 & 1.31956500 & 1.31642800 & -0.15958000 \\
\hline 0 & -2.97635500 & 2.67195100 & -1.94859400 \\
\hline 0 & -0.52014100 & 3.64084800 & -1.06528600 \\
\hline 0 & -4.13700100 & 0.69350100 & -0.28976700 \\
\hline $\mathrm{C}$ & 1.82587900 & -1.72269200 & 1.84814200 \\
\hline 0 & 1.33467200 & -2.79438300 & 2.11107800 \\
\hline $\mathrm{C}$ & 3.24464600 & -1.39704800 & 1.42145900 \\
\hline C & 4.03109800 & -1.80588300 & -0.90529900 \\
\hline $\mathrm{C}$ & 3.25352300 & -0.77075500 & -0.03653100 \\
\hline $\mathrm{C}$ & 4.09115700 & 0.48390900 & 0.02163400 \\
\hline $\mathrm{C}$ & 5.45414700 & 0.21884000 & 0.21587700 \\
\hline C & 6.39621200 & 1.24499700 & 0.31765800 \\
\hline C & 5.96492600 & 2.57908700 & 0.26049700 \\
\hline $\mathrm{C}$ & 4.61116300 & 2.86022900 & 0.12165900 \\
\hline $\mathrm{C}$ & 3.65827400 & 1.83079200 & 0.00971600 \\
\hline C & 2.23995800 & 2.28701500 & 0.00040600 \\
\hline 0 & 1.92675200 & 3.46506600 & 0.14789400 \\
\hline 0 & 7.72211500 & 1.01092300 & 0.48488100 \\
\hline 0 & 6.86292100 & 3.59567500 & 0.35810400 \\
\hline $\mathrm{C}$ & -4.78762200 & -0.48358200 & -0.55165400 \\
\hline 0 & -4.22309100 & -1.45892900 & -1.00998200 \\
\hline C & -6.22706700 & -0.41113200 & -0.22079800 \\
\hline $\mathrm{C}$ & -6.81165000 & 0.75320100 & 0.29832000 \\
\hline $\mathrm{C}$ & -8.17300700 & 0.76479400 & 0.59067700 \\
\hline C & -8.94640800 & -0.38218300 & 0.36581000 \\
\hline $\mathrm{C}$ & -8.35549000 & -1.54317800 & -0.15363500 \\
\hline $\mathrm{C}$ & -6.99823800 & -1.56324700 & -0.44817600 \\
\hline 0 & -9.21933100 & -2.58991800 & -0.32565600 \\
\hline 0 & -10.27266300 & -0.31671000 & 0.66975800 \\
\hline 0 & -8.75092900 & 1.89144100 & 1.09500300 \\
\hline $\mathrm{C}$ & 5.09643000 & -2.23504700 & 0.13703400 \\
\hline C & 4.30064300 & -2.52134700 & 1.41032000 \\
\hline 0 & 5.96248700 & -1.05439200 & 0.33305400 \\
\hline 0 & 4.65570400 & -1.30283900 & -2.08071600 \\
\hline 0 & 5.92884500 & -3.26632400 & -0.22003600 \\
\hline $\mathrm{H}$ & -2.51584400 & 0.09060900 & -1.40996200 \\
\hline $\mathrm{H}$ & -0.42141300 & -0.49987100 & -0.33903400 \\
\hline $\mathrm{H}$ & -0.09212700 & 2.19075400 & 1.10534600 \\
\hline $\mathrm{H}$ & -0.54671000 & 1.72629900 & -1.87028200 \\
\hline $\mathrm{H}$ & -2.62993300 & 2.75376300 & 0.10655500 \\
\hline $\mathrm{H}$ & -0.68703400 & -1.48146500 & 1.90491200 \\
\hline $\mathrm{H}$ & -0.61073400 & 0.13152400 & 2.65964200 \\
\hline $\mathrm{H}$ & -2.57807100 & 3.53848300 & -2.12165300 \\
\hline $\mathrm{H}$ & 0.37317800 & 3.79490800 & -0.70001000 \\
\hline $\mathrm{H}$ & 3.55721100 & -0.60582600 & 2.10635800 \\
\hline $\mathrm{H}$ & 2.26090800 & -0.57970800 & -0.42214700 \\
\hline $\mathrm{H}$ & 4.28411400 & 3.89211600 & 0.11914600 \\
\hline $\mathrm{H}$ & 7.86825800 & 0.05148500 & 0.52048200 \\
\hline $\mathrm{H}$ & 7.74839500 & 3.21523900 & 0.47005500 \\
\hline $\mathrm{H}$ & -6.22361400 & 1.64423100 & 0.47538600 \\
\hline $\mathrm{H}$ & -6.52662700 & -2.45345700 & -0.84968000 \\
\hline $\mathrm{H}$ & -8.75551000 & -3.35771100 & -0.68707200 \\
\hline $\mathrm{H}$ & -10.68041200 & -1.17355700 & 0.46685200 \\
\hline $\mathrm{H}$ & -9.69433100 & 1.71267000 & 1.23338000 \\
\hline $\mathrm{H}$ & 3.83066700 & -3.50141300 & 1.32714000 \\
\hline $\mathrm{H}$ & 4.94971600 & -2.50230800 & 2.28744300 \\
\hline $\mathrm{H}$ & 4.62753100 & -0.33347300 & -2.08660400 \\
\hline $\mathrm{H}$ & 6.34152200 & -3.03571800 & -1.06861000 \\
\hline $\mathrm{C}$ & 3.12728400 & -2.97075200 & -1.36954900 \\
\hline 0 & 2.47398200 & -3.66230900 & -0.61865400 \\
\hline 0 & 3.09810500 & -3.14742900 & -2.69282400 \\
\hline $\mathrm{H}$ & 3.68241500 & -2.46099800 & -3.08051500 \\
\hline
\end{tabular}


Cartesian coordinates of the lowest-energy conformer of $\mathbf{1}^{\prime} \mathbf{A}\left(\mathbf{1}^{\prime} \mathbf{A}-\mathbf{1}\right)$ at the B3LYP/6-31G(d,p) level in $\mathrm{MeOH}(\mathrm{PCM})$.

\begin{tabular}{|c|c|c|c|}
\hline C & 3.19810500 & -1.45977800 & -0.33132300 \\
\hline 0 & 2.21444100 & -0.44722200 & -0.48799600 \\
\hline C & 0.94479100 & -1.01589100 & -0.82383600 \\
\hline$c$ & 0.46005400 & -1.81819100 & 0.39858000 \\
\hline C & 1.42228400 & -2.97043500 & 0.65344300 \\
\hline C & 2.83509000 & -2.39259800 & 0.82832500 \\
\hline $\mathrm{C}$ & -0.00759200 & 0.07259300 & -1.30386300 \\
\hline 0 & -0.73387600 & 0.65884100 & -0.18700700 \\
\hline 0 & -0.87867800 & -2.28119900 & 0.13533300 \\
\hline 0 & 3.71673400 & -3.50146400 & 0.91377800 \\
\hline $\mathrm{O}$ & 1.02490500 & -3.66876200 & 1.81836100 \\
\hline O & 4.40829900 & -0.81377600 & 0.00926300 \\
\hline c & -1.92085200 & 1.20404900 & -0.46835600 \\
\hline 0 & -2.33004900 & 1.32711300 & -1.61781700 \\
\hline 0 & -2.68932100 & 1.64267100 & 0.76883800 \\
\hline $\mathrm{C}$ & -5.18235700 & 1.36163500 & 0.97857500 \\
\hline C & -3.81777900 & 0.63010400 & 1.22656800 \\
\hline $\mathrm{C}$ & -4.01903000 & -0.68240900 & 0.51639200 \\
\hline $\mathrm{C}$ & -5.31525600 & -0.69655900 & 0.00997800 \\
\hline c & -5.81414800 & -1.76889600 & -0.72031500 \\
\hline C & -4.96197200 & -2.86727300 & -0.94493100 \\
\hline C & -3.66141500 & -2.87744800 & -0.43821400 \\
\hline C & -3.19251100 & -1.79477700 & 0.32216300 \\
\hline C & -1.84608200 & -1.76145200 & 0.94165100 \\
\hline 0 & -1.61975900 & -1.24945500 & 2.02119200 \\
\hline 0 & -7.07530100 & -1.83522700 & -1.23706900 \\
\hline 0 & -5.41086800 & -3.93470900 & -1.66210800 \\
\hline $\mathrm{C}$ & 5.19225200 & -0.36320500 & -1.02423600 \\
\hline 0 & 4.89654500 & -0.53146300 & -2.19147200 \\
\hline C & 6.40789500 & 0.32616900 & -0.54295600 \\
\hline $\mathrm{C}$ & 6.67640200 & 0.49217600 & 0.82370000 \\
\hline C & 7.83757400 & 1.15117900 & 1.21913500 \\
\hline C & 8.72634300 & 1.64277700 & 0.25326500 \\
\hline $\mathrm{C}$ & 8.45114500 & 1.47418500 & -1.11151500 \\
\hline C & 7.29563100 & 0.81780200 & -1.51464300 \\
\hline O & 9.39533300 & 1.99929900 & -1.95006400 \\
\hline $\mathrm{O}$ & 9.84626200 & 2.27986300 & 0.69435600 \\
\hline 0 & 8.10716700 & 1.31782200 & 2.54452000 \\
\hline C & -4.85018800 & 2.55643300 & 0.00858200 \\
\hline C & -3.45420800 & 2.97254500 & 0.51888800 \\
\hline 0 & -6.05640600 & 0.41375500 & 0.30251100 \\
\hline 0 & -5.73368500 & 1.75382500 & 2.17731900 \\
\hline 0 & -4.84139300 & 2.13859100 & -1.34328400 \\
\hline $\mathrm{C}$ & -5.86967200 & 3.70474600 & 0.07995200 \\
\hline 0 & -6.33961300 & 4.12539200 & 1.12808400 \\
\hline 0 & -6.20019700 & 4.21988300 & -1.09447400 \\
\hline $\mathrm{H}$ & 3.32492500 & -2.01652800 & -1.26752100 \\
\hline $\mathrm{H}$ & 1.07312300 & -1.69697000 & -1.67932900 \\
\hline $\mathrm{H}$ & 0.43487700 & -1.16681100 & 1.27252400 \\
\hline $\mathrm{H}$ & 1.42494900 & -3.64279800 & -0.21993900 \\
\hline $\mathrm{H}$ & 2.84838600 & -1.79952100 & 1.75316100 \\
\hline $\mathrm{H}$ & -0.73420900 & -0.34747700 & -1.99821700 \\
\hline $\mathrm{H}$ & 0.55655900 & 0.85761800 & -1.81203600 \\
\hline $\mathrm{H}$ & 4.55806400 & -3.18943200 & 1.27481000 \\
\hline $\mathrm{H}$ & 1.73834700 & -4.29640800 & 2.00997700 \\
\hline $\mathrm{H}$ & -1.96452700 & 1.75649400 & 1.57449800 \\
\hline $\mathrm{H}$ & -3.70360700 & 0.47921400 & 2.30142100 \\
\hline $\mathrm{H}$ & -3.03459500 & -3.74211100 & -0.62111200 \\
\hline $\mathrm{H}$ & -7.54740900 & -1.01028800 & -1.04980400 \\
\hline $\mathrm{H}$ & -6.32452100 & -3.75582000 & -1.93533500 \\
\hline $\mathrm{H}$ & 5.99611500 & 0.11931800 & 1.57816800 \\
\hline $\mathrm{H}$ & 7.07136300 & 0.68075200 & -2.56671600 \\
\hline $\mathrm{H}$ & 9.14278800 & 1.85963800 & -2.87316000 \\
\hline $\mathrm{H}$ & 10.36233100 & 2.56939200 & -0.07477300 \\
\hline $\mathrm{H}$ & 8.94693900 & 1.79673200 & 2.62557300 \\
\hline $\mathrm{H}$ & -2.94898100 & 3.64171400 & -0.18119600 \\
\hline $\mathrm{H}$ & -3.56988300 & 3.49751200 & 1.46996600 \\
\hline $\mathrm{H}$ & -6.17687300 & 2.62100800 & 2.05504500 \\
\hline $\mathrm{H}$ & -3.92676900 & 1.87343600 & -1.60389600 \\
\hline $\mathrm{H}$ & -5.73738300 & 3.66156900 & -1.76259800 \\
\hline
\end{tabular}


Cartesian coordinates of the lowest-energy conformer of $\mathbf{1}^{\prime} \mathbf{B}\left(\mathbf{1}^{\prime} \mathbf{B}-\mathbf{1}\right)$ at the B3LYP/6-31G(d,p) level in $\mathrm{MeOH}(\mathrm{PCM})$.

\begin{tabular}{|c|c|c|c|}
\hline C & -3.13608300 & 1.49327300 & -0.23116500 \\
\hline 0 & -2.14599500 & 0.48991400 & -0.41785800 \\
\hline C & -0.87406700 & 1.05460300 & -0.74430500 \\
\hline$c$ & -0.38614100 & 1.83580500 & 0.49051900 \\
\hline $\mathrm{C}$ & -1.36375400 & 2.96692400 & 0.78785500 \\
\hline C & -2.77833900 & 2.37887300 & 0.96326600 \\
\hline C & 0.07724500 & -0.03995700 & -1.21730300 \\
\hline 0 & 0.76708100 & -0.63052700 & -0.07910300 \\
\hline 0 & 0.94099400 & 2.31942200 & 0.21810000 \\
\hline 0 & -3.73711100 & 3.41205300 & 1.07001800 \\
\hline 0 & -1.01047800 & 3.75286300 & 1.91658200 \\
\hline 0 & -4.34174600 & 0.82051700 & 0.06154400 \\
\hline C & 1.94906400 & -1.20349100 & -0.30294600 \\
\hline 0 & 2.40361600 & -1.35783300 & -1.43318300 \\
\hline C & 2.66326500 & -1.60601500 & 0.96947900 \\
\hline C & 5.17264100 & -1.37699200 & 1.13116000 \\
\hline C & 3.83247400 & -0.61158300 & 1.35253400 \\
\hline C & 4.02619200 & 0.64581300 & 0.54367400 \\
\hline C & 5.22821200 & 0.52620100 & -0.14549900 \\
\hline C & 5.69555800 & 1.50887700 & -1.00963600 \\
\hline $\mathrm{C}$ & 4.89348600 & 2.65076200 & -1.19998800 \\
\hline C & 3.66837900 & 2.77761000 & -0.54464500 \\
\hline C & 3.23621700 & 1.78460700 & 0.34872000 \\
\hline C & 1.92204900 & 1.83546600 & 1.03162300 \\
\hline 0 & 1.71169200 & 1.40155800 & 2.14807200 \\
\hline 0 & 6.87035900 & 1.44541900 & -1.69788000 \\
\hline 0 & 5.31100000 & 3.63781700 & -2.03884800 \\
\hline $\mathrm{C}$ & -5.11509000 & 0.43385800 & -1.00239900 \\
\hline 0 & -4.81162200 & 0.67170000 & -2.15611700 \\
\hline $\mathrm{C}$ & -6.33503400 & -0.28350300 & -0.57454500 \\
\hline C & -6.62105600 & -0.52306500 & 0.77742100 \\
\hline C & -7.78567400 & -1.20457500 & 1.12147100 \\
\hline $\mathrm{C}$ & -8.66057800 & -1.64494300 & 0.11897300 \\
\hline C & -8.36841800 & -1.40171200 & -1.23086800 \\
\hline $\mathrm{C}$ & -7.20908400 & -0.72299200 & -1.58281500 \\
\hline 0 & -9.30066800 & -1.88167200 & -2.10922500 \\
\hline 0 & -9.78492500 & -2.30686400 & 0.51029600 \\
\hline 0 & -8.07260400 & -1.44232300 & 2.43243400 \\
\hline $\mathrm{C}$ & 4.84541800 & -2.84287000 & 0.62164600 \\
\hline $\mathrm{C}$ & 3.32590600 & -3.00566200 & 0.86055200 \\
\hline 0 & 5.90176100 & -0.64012800 & 0.08969900 \\
\hline 0 & 5.93359400 & -1.42321000 & 2.27160700 \\
\hline 0 & 5.56454000 & -3.77126300 & 1.41381100 \\
\hline $\mathrm{C}$ & 5.33669100 & -3.06677700 & -0.83571700 \\
\hline 0 & 6.34860300 & -3.73056500 & -1.00578100 \\
\hline 0 & 4.67043400 & -2.56630400 & -1.85363200 \\
\hline $\mathrm{H}$ & -3.25367800 & 2.08788400 & -1.14495500 \\
\hline $\mathrm{H}$ & -0.99034200 & 1.74593900 & -1.59299200 \\
\hline $\mathrm{H}$ & -0.33944600 & 1.15474500 & 1.34260000 \\
\hline $\mathrm{H}$ & -1.37984100 & 3.66802600 & -0.05470800 \\
\hline $\mathrm{H}$ & -2.78099200 & 1.73599300 & 1.85891600 \\
\hline $\mathrm{H}$ & 0.82490100 & 0.37559500 & -1.89210200 \\
\hline $\mathrm{H}$ & -0.48206700 & -0.82002300 & -1.73776700 \\
\hline $\mathrm{H}$ & -3.37072100 & 4.05023700 & 1.70191000 \\
\hline $\mathrm{H}$ & -0.87306900 & 3.16207900 & 2.67287400 \\
\hline $\mathrm{H}$ & 1.92063600 & -1.59811500 & 1.76645600 \\
\hline $\mathrm{H}$ & 3.73898900 & -0.39339700 & 2.41677700 \\
\hline $\mathrm{H}$ & 3.06653600 & 3.65967700 & -0.72779900 \\
\hline $\mathrm{H}$ & 7.33248400 & 0.62106100 & -1.48495800 \\
\hline $\mathrm{H}$ & 6.16790400 & 3.37912200 & -2.41349700 \\
\hline $\mathrm{H}$ & -5.95277100 & -0.18722800 & 1.55950500 \\
\hline $\mathrm{H}$ & -6.97188700 & -0.52813000 & -2.62287900 \\
\hline $\mathrm{H}$ & -9.04047600 & -1.68381400 & -3.01944700 \\
\hline $\mathrm{H}$ & -10.29259400 & -2.55025600 & -0.28007200 \\
\hline $\mathrm{H}$ & -8.91400900 & -1.92317900 & 2.47646900 \\
\hline $\mathrm{H}$ & 2.84814200 & -3.64213400 & 0.11220300 \\
\hline $\mathrm{H}$ & 3.22655100 & -3.51371500 & 1.82217000 \\
\hline $\mathrm{H}$ & 6.24785900 & -2.35020100 & 2.33911100 \\
\hline $\mathrm{H}$ & 6.25716300 & -4.12107700 & 0.81239500 \\
\hline $\mathrm{H}$ & 3.82563400 & -2.08489000 & -1.61686700 \\
\hline
\end{tabular}


Cartesian coordinates of the lowest-energy conformer of $\mathbf{1}^{\prime} \mathbf{C}\left(\mathbf{1}^{\prime} \mathbf{C}-\mathbf{1}\right)$ at the B3LYP/6-31G(d,p) level in $\mathrm{MeOH}(\mathrm{PCM})$.

\begin{tabular}{|c|c|c|c|}
\hline C & -3.17072800 & 0.38243200 & -0.76383600 \\
\hline 0 & -2.48031100 & -0.36292400 & 0.23009500 \\
\hline $\mathrm{C}$ & -1.07719000 & -0.47983400 & -0.01163300 \\
\hline$c$ & -0.47001600 & 0.93181500 & 0.05243000 \\
\hline C & -1.13834600 & 1.81560700 & -1.00186500 \\
\hline $\mathrm{C}$ & -2.66039300 & 1.81941400 & -0.77599700 \\
\hline C & -0.54643400 & -1.44020100 & 1.05269100 \\
\hline O & 0.73131200 & -1.92812600 & 0.61289700 \\
\hline 0 & 0.96202500 & 0.86058900 & -0.16798000 \\
\hline 0 & -3.30809800 & 2.53730400 & -1.80737200 \\
\hline 0 & -0.65604200 & 3.15108100 & -1.04260100 \\
\hline 0 & -4.53005200 & 0.39152600 & -0.38260100 \\
\hline $\mathrm{C}$ & 1.85115100 & -1.44411900 & 1.19399300 \\
\hline 0 & 1.87557900 & -0.77335400 & 2.20539100 \\
\hline $\mathrm{C}$ & 3.05161800 & -1.85870500 & 0.38255000 \\
\hline C & 4.95963300 & -1.39049500 & -1.05017000 \\
\hline C & 3.54446900 & -0.81164000 & -0.66747900 \\
\hline C & 3.87508800 & 0.61253000 & -0.24581500 \\
\hline $\mathrm{C}$ & 5.23199600 & 0.79905100 & -0.51151100 \\
\hline $\mathrm{C}$ & 5.93058000 & 1.97173200 & -0.25372900 \\
\hline $\mathrm{C}$ & 5.21299100 & 3.05170000 & 0.28015500 \\
\hline C & 3.84859400 & 2.92132500 & 0.51295600 \\
\hline C & 3.15494300 & 1.72518500 & 0.23873900 \\
\hline $\mathrm{C}$ & 1.69145000 & 1.81780100 & 0.45928800 \\
\hline 0 & 1.16713900 & 2.71730700 & 1.10257500 \\
\hline 0 & 7.25876500 & 2.13931700 & -0.49713800 \\
\hline O & 5.84016000 & 4.22724500 & 0.55801600 \\
\hline $\mathrm{C}$ & -5.30844700 & -0.65340500 & -0.80917500 \\
\hline O & -4.87891400 & -1.55002100 & -1.50962700 \\
\hline $\mathrm{C}$ & -6.70094300 & -0.54242800 & -0.32362300 \\
\hline C & -7.59980500 & -1.55087400 & -0.69946600 \\
\hline C & -8.92138600 & -1.49293000 & -0.26934700 \\
\hline $\mathrm{C}$ & -9.34406900 & -0.42674600 & 0.53702600 \\
\hline C & -8.44110400 & 0.57841300 & 0.91064700 \\
\hline $\mathrm{C}$ & -7.11856900 & 0.52836900 & 0.48480800 \\
\hline 0 & -8.97943800 & 1.55931100 & 1.69805100 \\
\hline 0 & -10.64752100 & -0.41433000 & 0.93246500 \\
\hline 0 & -9.80164100 & -2.46860800 & -0.63032200 \\
\hline $\mathrm{C}$ & 5.37502500 & -2.40347100 & 0.12494500 \\
\hline $\mathrm{C}$ & 4.29992700 & -2.19132200 & 1.20253700 \\
\hline 0 & 5.87151200 & -0.27236600 & -1.07075400 \\
\hline 0 & 5.00147600 & -2.01625700 & -2.27064300 \\
\hline 0 & 5.30708500 & -3.71912400 & -0.40760900 \\
\hline $\mathrm{C}$ & 6.81023900 & -2.19692500 & 0.61789400 \\
\hline 0 & 7.69156400 & -2.98998500 & 0.33980600 \\
\hline O & 6.98122800 & -1.12156500 & 1.38362100 \\
\hline $\mathrm{H}$ & -3.06258500 & -0.09353700 & -1.74545800 \\
\hline $\mathrm{H}$ & -0.89284600 & -0.92315200 & -1.00122300 \\
\hline $\mathrm{H}$ & -0.64431000 & 1.33948100 & 1.05148600 \\
\hline $\mathrm{H}$ & -0.94226400 & 1.39198300 & -1.99578500 \\
\hline $\mathrm{H}$ & -2.87287100 & 2.25842300 & 0.21170500 \\
\hline $\mathrm{H}$ & -1.21144200 & -2.30278600 & 1.11700400 \\
\hline $\mathrm{H}$ & -0.45695700 & -0.96587600 & 2.03137900 \\
\hline $\mathrm{H}$ & -2.81135300 & 3.36598200 & -1.89710800 \\
\hline $\mathrm{H}$ & -0.35685200 & 3.38998800 & -0.14936600 \\
\hline $\mathrm{H}$ & 2.76038700 & -2.74165200 & -0.19418600 \\
\hline $\mathrm{H}$ & 2.89193400 & -0.82294600 & -1.54406200 \\
\hline $\mathrm{H}$ & 3.30077900 & 3.77044100 & 0.90095000 \\
\hline $\mathrm{H}$ & 7.63779000 & 1.30475300 & -0.81230800 \\
\hline $\mathrm{H}$ & 6.77808600 & 4.13611600 & 0.32770200 \\
\hline $\mathrm{H}$ & -7.26904500 & -2.37257500 & -1.32274100 \\
\hline $\mathrm{H}$ & -6.42064600 & 1.30483400 & 0.77379500 \\
\hline $\mathrm{H}$ & -8.31000100 & 2.22074400 & 1.92103300 \\
\hline $\mathrm{H}$ & -10.79840200 & 0.37382700 & 1.47805300 \\
\hline $\mathrm{H}$ & -10.66478600 & -2.25738800 & -0.24105100 \\
\hline $\mathrm{H}$ & 4.18908500 & -3.08679800 & 1.81738400 \\
\hline $\mathrm{H}$ & 4.56082900 & -1.35036400 & 1.84918900 \\
\hline $\mathrm{H}$ & 5.17095600 & -2.96079900 & -2.07321400 \\
\hline $\mathrm{H}$ & 6.21854200 & -4.06987800 & -0.36335400 \\
\hline $\mathrm{H}$ & 7.91770400 & -1.07866200 & 1.64796200 \\
\hline
\end{tabular}


Cartesian coordinates of the lowest-energy conformer of $\mathbf{1}^{\prime} \mathbf{D}\left(\mathbf{1}^{\prime} \mathbf{D}-\mathbf{1}\right)$ at the B3LYP/6-31G(d,p) level in $\mathrm{MeOH}(\mathrm{PCM})$.

\begin{tabular}{|c|c|c|c|}
\hline C & -3.06598400 & -0.33307600 & 0.80635800 \\
\hline 0 & -2.38811800 & 0.22814400 & -0.31033900 \\
\hline C & -0.97960900 & 0.35924900 & -0.11354200 \\
\hline$c$ & -0.39673400 & -1.05593300 & 0.03316400 \\
\hline C & -1.04651900 & -1.74700600 & 1.23221200 \\
\hline $\mathrm{C}$ & -2.57490200 & -1.75741100 & 1.04501600 \\
\hline C & -0.44229800 & 1.13384300 & -1.31766500 \\
\hline O & 0.82724100 & 1.68622800 & -0.93570600 \\
\hline 0 & 1.04519900 & -0.98386500 & 0.18742200 \\
\hline 0 & -3.20682700 & -2.28336600 & 2.19509300 \\
\hline 0 & -0.58343100 & -3.06792100 & 1.47246600 \\
\hline O & -4.43320500 & -0.38022200 & 0.45858900 \\
\hline $\mathrm{C}$ & 1.96309600 & 1.14175400 & -1.42821100 \\
\hline 0 & 2.01578900 & 0.37191800 & -2.36469100 \\
\hline $\mathrm{C}$ & 3.13203900 & 1.64136700 & -0.61763800 \\
\hline C & 5.08154900 & 1.24497900 & 0.79470800 \\
\hline C & 3.65799500 & 0.64942100 & 0.47598400 \\
\hline $\mathrm{C}$ & 3.96615900 & -0.79373000 & 0.10137800 \\
\hline $\mathrm{C}$ & 5.34305600 & -0.96302000 & 0.21970700 \\
\hline $\mathrm{C}$ & 6.02121700 & -2.15165600 & -0.01781100 \\
\hline $\mathrm{C}$ & 5.25629500 & -3.26351100 & -0.40257200 \\
\hline C & 3.87571500 & -3.14432600 & -0.50239600 \\
\hline C & 3.20546000 & -1.93305300 & -0.23882600 \\
\hline $\mathrm{C}$ & 1.72811800 & -2.03783100 & -0.32504800 \\
\hline 0 & 1.15811200 & -3.01774100 & -0.78605800 \\
\hline 0 & 7.36536100 & -2.31569200 & 0.09982900 \\
\hline O & 5.85445800 & -4.45739600 & -0.65777300 \\
\hline $\mathrm{C}$ & -5.18624700 & 0.73450800 & 0.72468300 \\
\hline 0 & -4.73015500 & 1.72043700 & 1.27139200 \\
\hline $\mathrm{C}$ & -6.58710100 & 0.57863300 & 0.27745100 \\
\hline $\mathrm{C}$ & -7.46223000 & 1.65001100 & 0.50692900 \\
\hline C & -8.79011500 & 1.55543900 & 0.10369800 \\
\hline $\mathrm{C}$ & -9.24302400 & 0.38982800 & -0.53025000 \\
\hline C & -8.36376800 & -0.67796600 & -0.75821000 \\
\hline $\mathrm{C}$ & -7.03532200 & -0.59219400 & -0.35720600 \\
\hline 0 & -8.92993400 & -1.75422200 & -1.38519700 \\
\hline 0 & -10.55110600 & 0.34552000 & -0.90752400 \\
\hline 0 & -9.64684800 & 2.59222600 & 0.32300600 \\
\hline $\mathrm{C}$ & 5.42774100 & 2.30063800 & -0.33358800 \\
\hline $\mathrm{C}$ & 4.37629300 & 2.02439000 & -1.42307300 \\
\hline 0 & 6.02923300 & 0.15238800 & 0.63896100 \\
\hline 0 & 5.25698700 & 1.82686400 & 2.03209300 \\
\hline 0 & 6.76503200 & 2.16906700 & -0.78833400 \\
\hline $\mathrm{C}$ & 5.30509800 & 3.74007600 & 0.22701600 \\
\hline 0 & 4.24854700 & 4.30150700 & 0.42513200 \\
\hline O & 6.47814700 & 4.32858600 & 0.46929900 \\
\hline $\mathrm{H}$ & -2.92864400 & 0.29511400 & 1.69418000 \\
\hline $\mathrm{H}$ & -0.77229300 & 0.94954000 & 0.79106600 \\
\hline $\mathrm{H}$ & -0.61285200 & -1.61271100 & -0.88204000 \\
\hline $\mathrm{H}$ & -0.81732200 & -1.17604700 & 2.14160400 \\
\hline $\mathrm{H}$ & -2.81834000 & -2.34969700 & 0.14870000 \\
\hline $\mathrm{H}$ & -1.10797200 & 1.97207000 & -1.52965300 \\
\hline $\mathrm{H}$ & -0.33680400 & 0.50987100 & -2.20698700 \\
\hline $\mathrm{H}$ & -2.72392200 & -3.09807600 & 2.40612400 \\
\hline $\mathrm{H}$ & -0.32740900 & -3.45538900 & 0.61882700 \\
\hline $\mathrm{H}$ & 2.79585700 & 2.52775500 & -0.07842200 \\
\hline $\mathrm{H}$ & 3.01232200 & 0.69719300 & 1.35682700 \\
\hline $\mathrm{H}$ & 3.29511600 & -4.01589400 & -0.77551400 \\
\hline $\mathrm{H}$ & 7.77502200 & -1.50615500 & 0.43925400 \\
\hline $\mathrm{H}$ & 6.80924600 & -4.36149300 & -0.51459800 \\
\hline $\mathrm{H}$ & -7.10825400 & 2.54916800 & 0.99609400 \\
\hline $\mathrm{H}$ & -6.35718700 & -1.41851200 & -0.53326800 \\
\hline $\mathrm{H}$ & -8.27203200 & -2.44899700 & -1.52621900 \\
\hline $\mathrm{H}$ & -10.72211600 & -0.50982100 & -1.33274800 \\
\hline $\mathrm{H}$ & -10.51823900 & 2.34426500 & -0.02383600 \\
\hline $\mathrm{H}$ & 4.21251900 & 2.88708900 & -2.07188100 \\
\hline $\mathrm{H}$ & 4.71605300 & 1.18745300 & -2.04151300 \\
\hline $\mathrm{H}$ & 4.99031100 & 1.20346300 & 2.72489000 \\
\hline $\mathrm{H}$ & 7.05857800 & 1.27037600 & -0.55836300 \\
\hline $\mathrm{H}$ & 7.16662300 & 3.69921500 & 0.16257900 \\
\hline
\end{tabular}


Cartesian coordinates of the lowest-energy conformer of $\mathbf{1}^{\prime} \mathbf{E}\left(\mathbf{1}^{\prime} \mathbf{E}-\mathbf{1}\right)$ at the B3LYP/6-31G(d,p) level in $\mathrm{MeOH}(\mathrm{PCM})$.

\begin{tabular}{|c|c|c|c|}
\hline C & -3.26324800 & 1.00625700 & -0.40279500 \\
\hline 0 & -2.42708100 & -0.01244700 & 0.11910400 \\
\hline C & -1.03858500 & 0.20710100 & -0.14524900 \\
\hline$c$ & -0.59580100 & 1.52349000 & 0.53999500 \\
\hline $\mathrm{C}$ & -1.49035400 & 2.68387900 & 0.11452100 \\
\hline $\mathrm{C}$ & -2.96496800 & 2.32390700 & 0.30652500 \\
\hline $\mathrm{C}$ & -0.35767000 & -1.06422100 & 0.36717600 \\
\hline 0 & 0.98420800 & -1.09899800 & -0.15920600 \\
\hline 0 & 0.74630600 & 1.86750900 & 0.12713400 \\
\hline 0 & -3.71188700 & 3.40756700 & -0.22445300 \\
\hline 0 & -1.14814300 & 3.82458100 & 0.88173600 \\
\hline 0 & -4.59460700 & 0.64287700 & -0.09812000 \\
\hline $\mathrm{C}$ & 1.87737300 & -1.88145000 & 0.48543700 \\
\hline 0 & 1.59156800 & -2.63560000 & 1.39192700 \\
\hline C & 3.27302400 & -1.64895700 & -0.05480600 \\
\hline $\mathrm{C}$ & 5.58028600 & -1.07997300 & 0.66357900 \\
\hline C & 4.08692600 & -0.64491200 & 0.82936300 \\
\hline $\mathrm{C}$ & 4.09545300 & 0.78833900 & 0.38550500 \\
\hline $\mathrm{C}$ & 5.36031600 & 1.07499400 & -0.11292400 \\
\hline $\mathrm{C}$ & 5.68273100 & 2.30535000 & -0.67131700 \\
\hline $\mathrm{C}$ & 4.66373500 & 3.27603800 & -0.74335500 \\
\hline C & 3.38115800 & 3.00251200 & -0.26520500 \\
\hline C & 3.09379900 & 1.76075200 & 0.32277800 \\
\hline C & 1.76484900 & 1.44633800 & 0.90816400 \\
\hline 0 & 1.61168700 & 0.83993300 & 1.95399500 \\
\hline 0 & 6.90659200 & 2.64497200 & -1.16847500 \\
\hline 0 & 4.93170400 & 4.49706300 & -1.28326200 \\
\hline $\mathrm{C}$ & -5.24404800 & -0.17104800 & -0.99327100 \\
\hline 0 & -4.72642800 & -0.55308600 & -2.02494700 \\
\hline C & -6.61506300 & -0.50333500 & -0.55256400 \\
\hline $\mathrm{C}$ & -7.13691400 & -0.04067400 & 0.66433400 \\
\hline C & -8.43540100 & -0.38665600 & 1.02903400 \\
\hline $\mathrm{C}$ & -9.20873000 & -1.19209200 & 0.18187900 \\
\hline C & -8.68048300 & -1.65198500 & -1.03325900 \\
\hline $\mathrm{C}$ & -7.38645600 & -1.31107900 & -1.40500900 \\
\hline 0 & -9.53638700 & -2.42791600 & -1.76550900 \\
\hline 0 & -10.47171100 & -1.50121200 & 0.58695000 \\
\hline 0 & -8.95166300 & 0.05775600 & 2.20931900 \\
\hline $\mathrm{C}$ & 5.56138400 & -2.31040700 & -0.32138400 \\
\hline $\mathrm{C}$ & 4.16265200 & -2.90443900 & -0.07295400 \\
\hline 0 & 6.25929400 & 0.03403800 & -0.01623700 \\
\hline 0 & 6.16625500 & -1.31868400 & 1.88031800 \\
\hline 0 & 5.60532000 & -1.87603600 & -1.67704400 \\
\hline $\mathrm{C}$ & 6.69614400 & -3.32393700 & -0.10163600 \\
\hline 0 & 7.09864400 & -3.65616200 & 1.00345900 \\
\hline 0 & 7.20394700 & -3.83724500 & -1.21424000 \\
\hline $\mathrm{H}$ & -3.13896800 & 1.09040200 & -1.48882200 \\
\hline $\mathrm{H}$ & -0.86379200 & 0.29564400 & -1.22812300 \\
\hline $\mathrm{H}$ & -0.62133700 & 1.40766200 & 1.62586500 \\
\hline $\mathrm{H}$ & -1.32685100 & 2.87605000 & -0.95849500 \\
\hline $\mathrm{H}$ & -3.16292200 & 2.18537900 & 1.37831500 \\
\hline $\mathrm{H}$ & -0.91251700 & -1.93132100 & -0.00054400 \\
\hline $\mathrm{H}$ & -0.32800200 & -1.09462400 & 1.45736900 \\
\hline $\mathrm{H}$ & -4.62591200 & 3.31959500 & 0.07930400 \\
\hline $\mathrm{H}$ & -1.81556100 & 4.49532100 & 0.67083800 \\
\hline $\mathrm{H}$ & 3.19260500 & -1.24699200 & -1.06588900 \\
\hline $\mathrm{H}$ & 3.81121600 & -0.76858700 & 1.87843700 \\
\hline $\mathrm{H}$ & 2.62263600 & 3.77351200 & -0.33200700 \\
\hline $\mathrm{H}$ & 7.54216600 & 1.93384100 & -1.00048200 \\
\hline $\mathrm{H}$ & 5.86574300 & 4.51711200 & -1.54516300 \\
\hline $\mathrm{H}$ & -6.54799100 & 0.57870600 & 1.32807900 \\
\hline $\mathrm{H}$ & -6.96529300 & -1.65842200 & -2.34204800 \\
\hline $\mathrm{H}$ & -9.11308100 & -2.72075800 & -2.58426700 \\
\hline $\mathrm{H}$ & -10.88548300 & -2.06199100 & -0.08834500 \\
\hline $\mathrm{H}$ & -9.85722400 & -0.28057900 & 2.29099900 \\
\hline $\mathrm{H}$ & 3.88840800 & -3.61738600 & -0.85385100 \\
\hline $\mathrm{H}$ & 4.13360600 & -3.41088800 & 0.89588400 \\
\hline $\mathrm{H}$ & 6.70956000 & -2.13429700 & 1.82596400 \\
\hline $\mathrm{H}$ & 6.15507100 & -1.07229400 & -1.69738000 \\
\hline $\mathrm{H}$ & 6.77032100 & -3.36909600 & -1.96060300 \\
\hline
\end{tabular}


Cartesian coordinates of the lowest-energy conformer of $\mathbf{1}^{\prime} \mathbf{F}\left(\mathbf{1}^{\prime} \mathbf{F}-\mathbf{1}\right)$ at the B3LYP/6-31G(d,p) level in $\mathrm{MeOH}(\mathrm{PCM})$.

\begin{tabular}{|c|c|c|c|}
\hline C & 3.21584600 & 1.04434100 & 0.43272200 \\
\hline 0 & 2.39969800 & 0.02756600 & -0.12635000 \\
\hline C & 1.00157900 & 0.20045000 & 0.12317500 \\
\hline C & 0.55044900 & 1.51771300 & -0.54823400 \\
\hline C & 1.41034900 & 2.67921000 & -0.05034300 \\
\hline $\mathrm{C}$ & 2.90036000 & 2.38096500 & -0.22941000 \\
\hline C & 0.36460500 & -1.07897200 & -0.42206600 \\
\hline O & -0.99103800 & -1.14855000 & 0.06497300 \\
\hline O & -0.80910600 & 1.84282900 & -0.17714800 \\
\hline 0 & 3.68622900 & 3.39264000 & 0.37050400 \\
\hline 0 & 1.15829300 & 3.88404900 & -0.76028900 \\
\hline 0 & 4.55030100 & 0.69891300 & 0.12739400 \\
\hline $\mathrm{C}$ & -1.84154200 & -1.95277500 & -0.61094900 \\
\hline 0 & -1.50268800 & -2.68834600 & -1.51480900 \\
\hline $\mathrm{C}$ & -3.26198700 & -1.77249700 & -0.11381700 \\
\hline C & -5.54778300 & -1.23663300 & -0.91355200 \\
\hline C & -4.06651100 & -0.77155400 & -1.01659400 \\
\hline C & -4.11824300 & 0.64710000 & -0.52480900 \\
\hline C & -5.39196200 & 0.86547200 & -0.01099400 \\
\hline $\mathrm{C}$ & -5.75228000 & 2.05761500 & 0.60530200 \\
\hline $\mathrm{C}$ & -4.76843700 & 3.05838100 & 0.72159800 \\
\hline C & -3.47805200 & 2.85126600 & 0.23171300 \\
\hline C & -3.14824700 & 1.64737600 & -0.41218000 \\
\hline C & -1.80202600 & 1.39406300 & -0.98347500 \\
\hline 0 & -1.60357500 & 0.80929700 & -2.03310700 \\
\hline 0 & -6.98800400 & 2.32850700 & 1.11665500 \\
\hline O & -5.07762400 & 4.24406700 & 1.31742600 \\
\hline C & 5.19752600 & -0.12956500 & 1.00622200 \\
\hline 0 & 4.67878400 & -0.53906700 & 2.02761600 \\
\hline C & 6.57327700 & -0.44519000 & 0.56550200 \\
\hline $\mathrm{C}$ & 7.10433600 & 0.06030600 & -0.63007700 \\
\hline C & 8.40608400 & -0.27178500 & -0.99628100 \\
\hline C & 9.17324000 & -1.10633600 & -0.17211400 \\
\hline C & 8.63610900 & -1.60811400 & 1.02222500 \\
\hline $\mathrm{C}$ & 7.33884500 & -1.28123900 & 1.39544600 \\
\hline 0 & 9.48679700 & -2.40891600 & 1.73390900 \\
\hline O & 10.43973900 & -1.40087100 & -0.57818000 \\
\hline 0 & 8.93198300 & 0.21460100 & -2.15573900 \\
\hline C & -5.56701500 & -2.60758300 & -0.11552200 \\
\hline $\mathrm{C}$ & -4.09458900 & -3.06272100 & -0.15708000 \\
\hline 0 & -6.24957900 & -0.19331400 & -0.15201600 \\
\hline 0 & -6.14237600 & -1.39054800 & -2.14075400 \\
\hline 0 & -6.41676300 & -3.52729300 & -0.77230400 \\
\hline $\mathrm{C}$ & -6.12238600 & -2.41820000 & 1.30189400 \\
\hline 0 & -7.21897500 & -2.84134900 & 1.61517700 \\
\hline 0 & -5.29565600 & -1.78416000 & 2.13256400 \\
\hline $\mathrm{H}$ & 3.08287800 & 1.09234200 & 1.51986900 \\
\hline $\mathrm{H}$ & 0.80805800 & 0.26522400 & 1.20462700 \\
\hline $\mathrm{H}$ & 0.61951500 & 1.42826300 & -1.63464400 \\
\hline $\mathrm{H}$ & 1.21850700 & 2.82080800 & 1.02382100 \\
\hline $\mathrm{H}$ & 3.11733400 & 2.28647900 & -1.30442100 \\
\hline $\mathrm{H}$ & 0.92735000 & -1.93911600 & -0.04980400 \\
\hline $\mathrm{H}$ & 0.36694900 & -1.09553300 & -1.51292500 \\
\hline $\mathrm{H}$ & 3.31107600 & 4.23316100 & 0.06556600 \\
\hline $\mathrm{H}$ & 0.30315000 & 4.22976600 & -0.46956400 \\
\hline $\mathrm{H}$ & -3.22375700 & -1.37866100 & 0.90241200 \\
\hline $\mathrm{H}$ & -3.74137700 & -0.86363700 & -2.05460400 \\
\hline $\mathrm{H}$ & -2.74686900 & 3.64370100 & 0.33906200 \\
\hline $\mathrm{H}$ & -7.58323400 & 1.58371900 & 0.94573900 \\
\hline $\mathrm{H}$ & -6.00931200 & 4.21565400 & 1.58666200 \\
\hline $\mathrm{H}$ & 6.52041500 & 0.70424300 & -1.27462300 \\
\hline $\mathrm{H}$ & 6.91053900 & -1.66109600 & 2.31651800 \\
\hline $\mathrm{H}$ & 9.05923600 & -2.72400800 & 2.54211600 \\
\hline $\mathrm{H}$ & 10.85009800 & -1.98083100 & 0.08280700 \\
\hline $\mathrm{H}$ & 9.83989400 & -0.11692700 & -2.23914500 \\
\hline $\mathrm{H}$ & -3.84492900 & -3.75987600 & 0.64570300 \\
\hline $\mathrm{H}$ & -3.93754300 & -3.57172500 & -1.11280800 \\
\hline $\mathrm{H}$ & -6.67654000 & -2.20916400 & -2.07796400 \\
\hline $\mathrm{H}$ & -7.20093100 & -3.62759100 & -0.19824700 \\
\hline $\mathrm{H}$ & -5.73130000 & -1.70219700 & 2.99988300 \\
\hline
\end{tabular}


Cartesian coordinates of the lowest-energy conformer of $\mathbf{1}^{\prime} \mathbf{G}\left(\mathbf{1}^{\prime} \mathbf{G}-\mathbf{1}\right)$ at the B3LYP/6-31G(d,p) level in $\mathrm{MeOH}(\mathrm{PCM})$.

\begin{tabular}{|c|c|c|c|}
\hline C & 3.04547800 & 0.44611700 & 0.63158700 \\
\hline O & 2.37885300 & -0.18359700 & -0.45292600 \\
\hline C & 0.96378600 & -0.29706200 & -0.28188900 \\
\hline$c$ & 0.36448800 & 1.11918700 & -0.20028200 \\
\hline C & 1.04014500 & 1.88999000 & 0.93650200 \\
\hline $\mathrm{C}$ & 2.56558000 & 1.88807900 & 0.74481600 \\
\hline C & 0.51081900 & -1.11166700 & -1.49886000 \\
\hline O & -0.92426700 & -1.15698500 & -1.61560000 \\
\hline 0 & -1.06236200 & 1.02135400 & 0.04920900 \\
\hline 0 & 3.19720500 & 2.51140500 & 1.84547900 \\
\hline 0 & 0.58041900 & 3.22559200 & 1.09025700 \\
\hline 0 & 4.41971200 & 0.45077300 & 0.30730000 \\
\hline $\mathrm{C}$ & -1.57240200 & -1.86507300 & -0.67113400 \\
\hline 0 & -1.01211800 & -2.56804000 & 0.14764700 \\
\hline $\mathrm{C}$ & -3.06910500 & -1.64292700 & -0.71765700 \\
\hline C & -4.84544300 & -1.37738800 & 1.00920800 \\
\hline C & -3.52752200 & -0.72840200 & 0.48293300 \\
\hline C & -3.93918500 & 0.69617100 & 0.17571500 \\
\hline C & -5.31303500 & 0.77476700 & 0.40011100 \\
\hline C & -6.07042500 & 1.92190600 & 0.19392500 \\
\hline $\mathrm{C}$ & -5.40427500 & 3.07140300 & -0.25888000 \\
\hline C & -4.03044800 & 3.03497700 & -0.46841400 \\
\hline C & -3.27523900 & 1.86573800 & -0.24536900 \\
\hline C & -1.81723900 & 2.02587100 & -0.45513800 \\
\hline 0 & -1.32090600 & 3.00203000 & -1.00481200 \\
\hline 0 & -7.41224100 & 2.00118000 & 0.40591700 \\
\hline O & -6.09391200 & 4.22468600 & -0.47861700 \\
\hline $\mathrm{C}$ & 5.15700900 & -0.64212900 & 0.68315500 \\
\hline 0 & 4.67746100 & -1.58300800 & 1.28637600 \\
\hline C & 6.57393800 & -0.52262300 & 0.27652500 \\
\hline $\mathrm{C}$ & 7.43578200 & -1.57485600 & 0.61744900 \\
\hline C & 8.77853600 & -1.51099000 & 0.25986800 \\
\hline C & 9.25934100 & -0.39544300 & -0.44013200 \\
\hline C & 8.39344800 & 0.65378600 & -0.77863600 \\
\hline $\mathrm{C}$ & 7.05005700 & 0.59826700 & -0.42458800 \\
\hline 0 & 8.98762800 & 1.68108600 & -1.45962000 \\
\hline O & 10.58125400 & -0.37959500 & -0.76860800 \\
\hline 0 & 9.62304400 & -2.52872000 & 0.58873300 \\
\hline C & -5.17680000 & -2.63036500 & 0.10802700 \\
\hline $\mathrm{C}$ & -3.81787800 & -2.97561800 & -0.51730600 \\
\hline 0 & -5.89754100 & -0.37425000 & 0.85266400 \\
\hline 0 & -4.74761300 & -1.75462800 & 2.32560600 \\
\hline 0 & -5.66079300 & -3.68593600 & 0.91514000 \\
\hline $\mathrm{C}$ & -6.26929300 & -2.30358900 & -0.91560200 \\
\hline 0 & -7.38719000 & -2.77417100 & -0.83016600 \\
\hline 0 & -5.86520600 & -1.48989100 & -1.89171300 \\
\hline $\mathrm{H}$ & 2.88467400 & -0.11125800 & 1.56156900 \\
\hline $\mathrm{H}$ & 0.73219100 & -0.84538000 & 0.63968500 \\
\hline $\mathrm{H}$ & 0.51654800 & 1.63454800 & -1.15258600 \\
\hline $\mathrm{H}$ & 0.81887500 & 1.37887400 & 1.88337100 \\
\hline $\mathrm{H}$ & 2.81073800 & 2.39849700 & -0.19988300 \\
\hline $\mathrm{H}$ & 0.91741000 & -2.12253600 & -1.43081600 \\
\hline $\mathrm{H}$ & 0.85833000 & -0.63455200 & -2.41593200 \\
\hline $\mathrm{H}$ & 2.70857800 & 3.33715900 & 1.99022300 \\
\hline $\mathrm{H}$ & 0.23717900 & 3.52769800 & 0.23252200 \\
\hline $\mathrm{H}$ & -3.34221400 & -1.17518500 & -1.66342600 \\
\hline $\mathrm{H}$ & -2.78516100 & -0.78211000 & 1.28241400 \\
\hline $\mathrm{H}$ & -3.52588900 & 3.93423400 & -0.79793500 \\
\hline $\mathrm{H}$ & -7.74119700 & 1.14912900 & 0.73043500 \\
\hline $\mathrm{H}$ & -7.02762200 & 4.06868100 & -0.26691000 \\
\hline $\mathrm{H}$ & 7.06028200 & -2.43466600 & 1.15857500 \\
\hline $\mathrm{H}$ & 6.38123300 & 1.40925800 & -0.68675600 \\
\hline $\mathrm{H}$ & 8.34182200 & 2.37198400 & -1.66176300 \\
\hline $\mathrm{H}$ & 10.77250200 & 0.44417300 & -1.24447100 \\
\hline $\mathrm{H}$ & 10.50848100 & -2.30608600 & 0.26079000 \\
\hline $\mathrm{H}$ & -3.27813700 & -3.58399800 & 0.21388600 \\
\hline $\mathrm{H}$ & -3.90836900 & -3.55149800 & -1.44084900 \\
\hline $\mathrm{H}$ & -5.26173200 & -2.58409400 & 2.40494800 \\
\hline $\mathrm{H}$ & -6.61403000 & -3.76292500 & 0.71792900 \\
\hline $\mathrm{H}$ & -6.61659800 & -1.32299100 & -2.48873000 \\
\hline
\end{tabular}


Cartesian coordinates of the lowest-energy conformer of $\mathbf{1}^{\prime} \mathbf{H}\left(\mathbf{1}^{\prime} \mathbf{H}-\mathbf{1}\right)$ at the B3LYP/6-31G(d,p) level in $\mathrm{MeOH}(\mathrm{PCM})$.

\begin{tabular}{|c|c|c|c|}
\hline C & -3.03915800 & 0.45199600 & -0.60334500 \\
\hline 0 & -2.38074900 & -0.16211100 & 0.49497800 \\
\hline $\mathrm{C}$ & -0.96098100 & -0.25238400 & 0.35284600 \\
\hline$c$ & -0.38253600 & 1.17341000 & 0.27299200 \\
\hline C & -1.05239300 & 1.92504600 & -0.88052800 \\
\hline $\mathrm{C}$ & -2.58045800 & 1.90078700 & -0.71596000 \\
\hline C & -0.52174200 & -1.05237900 & 1.58440200 \\
\hline O & 0.90996200 & -1.05700200 & 1.74018900 \\
\hline 0 & 1.04921600 & 1.09919900 & 0.04125100 \\
\hline 0 & -3.20126500 & 2.50703500 & -1.83207300 \\
\hline 0 & -0.60902700 & 3.26555500 & -1.03970700 \\
\hline 0 & -4.41828300 & 0.43762700 & -0.30083400 \\
\hline $\mathrm{C}$ & 1.60491200 & -1.75653500 & 0.82368200 \\
\hline 0 & 1.09389700 & -2.49204400 & 0.00172700 \\
\hline $\mathrm{C}$ & 3.08972000 & -1.47862000 & 0.90619600 \\
\hline C & 4.90818600 & -1.25368500 & -0.77986900 \\
\hline C & 3.56063500 & -0.60323100 & -0.31269300 \\
\hline C & 3.93487700 & 0.84330200 & -0.07725000 \\
\hline $\mathrm{C}$ & 5.29809500 & 0.96046000 & -0.34283300 \\
\hline $\mathrm{C}$ & 6.01751200 & 2.14307500 & -0.22494200 \\
\hline $\mathrm{C}$ & 5.32007400 & 3.28973300 & 0.18930300 \\
\hline C & 3.95597800 & 3.21282100 & 0.44597600 \\
\hline C & 3.23948800 & 2.00790400 & 0.30185900 \\
\hline $\mathrm{C}$ & 1.78003300 & 2.12559200 & 0.53713100 \\
\hline 0 & 1.26546800 & 3.08881100 & 1.09076600 \\
\hline 0 & 7.34782800 & 2.26415100 & -0.48079900 \\
\hline O & 5.97031200 & 4.47701200 & 0.32708800 \\
\hline $\mathrm{C}$ & -5.13449000 & -0.66524700 & -0.68524400 \\
\hline 0 & -4.63274900 & -1.60301700 & -1.27580200 \\
\hline $\mathrm{C}$ & -6.56028400 & -0.56300200 & -0.30608400 \\
\hline C & -7.06800800 & 0.55155900 & 0.37726800 \\
\hline C & -8.41998900 & 0.59608000 & 0.70743400 \\
\hline $\mathrm{C}$ & -9.26093300 & -0.46851700 & 0.35590400 \\
\hline C & -8.74669400 & -1.58002800 & -0.32732500 \\
\hline $\mathrm{C}$ & -7.39918800 & -1.63299300 & -0.65970800 \\
\hline 0 & -9.67048200 & -2.54825600 & -0.61110200 \\
\hline 0 & -10.57494600 & -0.37412000 & 0.70247900 \\
\hline 0 & -8.92300900 & 1.67478000 & 1.37151700 \\
\hline $\mathrm{C}$ & 5.28263900 & -2.31054700 & 0.31700200 \\
\hline $\mathrm{C}$ & 3.90274400 & -2.78574500 & 0.78150100 \\
\hline 0 & 5.91972300 & -0.19991500 & -0.74048100 \\
\hline 0 & 4.78652900 & -1.76676500 & -2.04596400 \\
\hline 0 & 5.91714700 & -1.68572500 & 1.42817800 \\
\hline $\mathrm{C}$ & 6.17934400 & -3.45698100 & -0.17956700 \\
\hline 0 & 6.06537200 & -3.97560700 & -1.28005100 \\
\hline O & 7.09034200 & -3.86719500 & 0.69381100 \\
\hline $\mathrm{H}$ & -2.85480700 & -0.10701100 & -1.52795800 \\
\hline $\mathrm{H}$ & -0.70097700 & -0.80221000 & -0.56048800 \\
\hline $\mathrm{H}$ & -0.55585500 & 1.69233800 & 1.21964400 \\
\hline $\mathrm{H}$ & -0.80675300 & 1.41059400 & -1.81940100 \\
\hline $\mathrm{H}$ & -2.85147700 & 2.41250700 & 0.22090400 \\
\hline $\mathrm{H}$ & -0.89863000 & -2.07409600 & 1.50868300 \\
\hline $\mathrm{H}$ & -0.90747700 & -0.58193200 & 2.48947200 \\
\hline $\mathrm{H}$ & -2.72223900 & 3.33887100 & -1.97418400 \\
\hline $\mathrm{H}$ & -0.30974400 & 3.59029700 & -0.17428300 \\
\hline $\mathrm{H}$ & 3.32088100 & -0.97134800 & 1.84315000 \\
\hline $\mathrm{H}$ & 2.84664800 & -0.71062300 & -1.13253800 \\
\hline $\mathrm{H}$ & 3.42737000 & 4.10885300 & 0.74543100 \\
\hline $\mathrm{H}$ & 7.69842400 & 1.42297200 & -0.81053300 \\
\hline $\mathrm{H}$ & 6.90143800 & 4.34756000 & 0.08754800 \\
\hline $\mathrm{H}$ & -6.42751100 & 1.37909100 & 0.65274600 \\
\hline $\mathrm{H}$ & -6.98805400 & -2.48635800 & -1.18771700 \\
\hline $\mathrm{H}$ & -9.25701500 & -3.28136800 & -1.08742700 \\
\hline $\mathrm{H}$ & -11.03472700 & -1.17184400 & 0.39611200 \\
\hline $\mathrm{H}$ & -9.87206800 & 1.52983100 & 1.51091300 \\
\hline $\mathrm{H}$ & 3.45712700 & -3.42869100 & 0.01795800 \\
\hline $\mathrm{H}$ & 3.96364600 & -3.33996200 & 1.72066600 \\
\hline $\mathrm{H}$ & 5.30629700 & -2.59783300 & -2.10647000 \\
\hline $\mathrm{H}$ & 6.50929200 & -1.00208200 & 1.06891900 \\
\hline $\mathrm{H}$ & 7.02962400 & -3.26808300 & 1.46861300 \\
\hline
\end{tabular}


Cartesian coordinates of the lowest-energy conformer of $\mathbf{2}(\mathbf{2}-\mathbf{A})$ at the B3LYP/6-311+G(2d,p) level in $\mathrm{MeOH}(\mathrm{PCM})$.

$\begin{array}{lrrr}\mathrm{C} & 1.90624400 & 0.92946700 & -0.39484500 \\ \mathrm{C} & 3.14891500 & 0.29212100 & -0.35514000 \\ \mathrm{C} & 3.27619500 & -1.05775000 & 0.01255900 \\ \mathrm{C} & 2.15100400 & -1.78363900 & 0.35426100 \\ \mathrm{C} & 0.91556500 & -1.13751000 & 0.30775600 \\ \mathrm{C} & 0.76810400 & 0.19988200 & -0.06646600 \\ \mathrm{C} & -0.38963700 & -1.72460600 & 0.58360200 \\ \mathrm{C} & -1.46946600 & -0.64257700 & 0.37995300 \\ \mathrm{C} & -0.69715300 & 0.61665800 & -0.11682500 \\ \mathrm{O} & -0.63828200 & -2.87170100 & 0.90853800 \\ \mathrm{O} & 1.92101600 & 2.23992300 & -0.76951700 \\ \mathrm{O} & 4.28876200 & 0.96248400 & -0.66417700 \\ \mathrm{O} & 4.50621900 & -1.64163600 & 0.04435100 \\ \mathrm{C} & -2.56049500 & -1.19614500 & -0.53955200 \\ \mathrm{C} & -0.99834300 & 1.93253700 & 0.61123400 \\ \mathrm{O} & -0.14929700 & 2.79589500 & 0.77121300 \\ \mathrm{O} & -2.22650000 & 2.15537400 & 1.03468100 \\ \mathrm{C} & -3.86215300 & -0.44802300 & -0.52100700 \\ \mathrm{O} & -4.09133300 & 0.56569900 & 0.11840700 \\ \mathrm{O} & -4.78844300 & -1.01831400 & -1.28678800 \\ \mathrm{H} & 2.23123600 & -2.82328600 & 0.64364800 \\ \mathrm{H} & -1.89781700 & -0.46089000 & 1.36935800 \\ \mathrm{H} & -0.97009800 & 0.82380100 & -1.16067900 \\ \mathrm{H} & 1.18419800 & 2.67655900 & -0.27811200 \\ \mathrm{H} & 4.06297100 & 1.89089400 & -0.83511900 \\ \mathrm{H} & 5.17539800 & -0.98941300 & -0.20929400 \\ \mathrm{H} & -2.78181700 & -2.22581600 & -0.24453100 \\ \mathrm{H} & -2.21902100 & -1.25058200 & -1.57753700 \\ \mathrm{H} & -2.88932900 & 1.46721700 & 0.74496100 \\ \mathrm{H} & -5.60554000 & -0.49277400 & -1.23952800 \\ & & & \end{array}$


Cartesian coordinates of the lowest-energy conformer of $\mathbf{3 A}(\mathbf{3 A - 1})$ at the B3LYP/6-31G(d,p) level in acetone (PCM).

\begin{tabular}{|c|c|c|c|}
\hline C & -2.71599500 & -1.49471900 & -0.12473200 \\
\hline O & -2.08960800 & -0.36401200 & -0.72137000 \\
\hline C & -0.77318000 & -0.08612600 & -0.23824800 \\
\hline C & 0.13214000 & -1.28333400 & -0.58829400 \\
\hline C & -0.45985800 & -2.54649900 & 0.04258600 \\
\hline C & -1.90562700 & -2.74698600 & -0.44305300 \\
\hline O & -2.48490900 & -3.86071200 & 0.20518600 \\
\hline O & 0.28730400 & -3.73201000 & -0.18633300 \\
\hline C & 3.09947600 & 2.10352300 & -0.03195700 \\
\hline C & 4.09720400 & -0.21921600 & 0.70221500 \\
\hline C & 5.34652200 & -0.15726400 & 1.31179600 \\
\hline C & 6.34721800 & -1.10222100 & 1.15301400 \\
\hline C & 6.06400700 & -2.19352000 & 0.30791200 \\
\hline C & 4.81465400 & -2.30274000 & -0.29475200 \\
\hline C & 3.80903100 & -1.33761400 & -0.09579500 \\
\hline O & 7.56977800 & -1.06187800 & 1.73984200 \\
\hline O & 7.00002200 & -3.15455500 & 0.09902700 \\
\hline C & 1.95493600 & 1.64436500 & -0.92112000 \\
\hline C & 2.48868400 & -1.64842100 & -0.69733400 \\
\hline 0 & 2.32970100 & -2.42773500 & -1.62369700 \\
\hline O & 2.08359800 & 1.18858700 & -2.03848900 \\
\hline O & 1.46048400 & -1.03257300 & -0.06369500 \\
\hline O & -3.98446000 & -1.63078800 & -0.70768200 \\
\hline C & -0.39682100 & 1.23891000 & -0.90261100 \\
\hline O & 0.77974400 & 1.78468900 & -0.27834500 \\
\hline C & 3.36605000 & 1.04524200 & 1.09374000 \\
\hline C & 4.33814500 & 1.68341500 & 2.09824200 \\
\hline 0 & 5.51073200 & 0.95130100 & 2.13244300 \\
\hline O & 4.20067300 & 2.68154600 & 2.75061500 \\
\hline C & 4.33482800 & 2.42692500 & -0.87408000 \\
\hline $\mathrm{C}$ & 4.09980600 & 3.62395900 & -1.77068800 \\
\hline O & 3.08757800 & 4.29559500 & -1.79316500 \\
\hline 0 & 5.16727400 & 3.87169400 & -2.55091700 \\
\hline $\mathrm{H}$ & 2.41823500 & 0.85289800 & 1.60405700 \\
\hline $\mathrm{H}$ & 2.76173200 & 3.00544000 & 0.48588400 \\
\hline $\mathrm{C}$ & -5.00998800 & -0.82343900 & -0.29462600 \\
\hline C & -4.84381400 & 0.29970600 & 0.50805700 \\
\hline C & -5.97269300 & 1.04205500 & 0.87459800 \\
\hline C & -7.29110300 & 0.68406900 & 0.43776000 \\
\hline C & -7.39450600 & -0.47038800 & -0.39392700 \\
\hline C & -6.27638200 & -1.21186000 & -0.75134000 \\
\hline O & -5.76922500 & 2.11457400 & 1.64740500 \\
\hline 0 & -8.62569000 & -0.83933700 & -0.82921100 \\
\hline $\mathrm{C}$ & -8.42003300 & 1.51431800 & 0.86396100 \\
\hline O & -8.22159200 & 2.51663300 & 1.58948000 \\
\hline C & -9.84771300 & 1.22274500 & 0.47074300 \\
\hline $\mathrm{H}$ & -2.80575300 & -1.35495000 & 0.96287500 \\
\hline $\mathrm{H}$ & -0.78251300 & 0.05425100 & 0.85296100 \\
\hline $\mathrm{H}$ & 0.19924200 & -1.38646400 & -1.67487000 \\
\hline $\mathrm{H}$ & -0.48553900 & -2.41065400 & 1.13220800 \\
\hline $\mathrm{H}$ & -1.90696500 & -2.87300600 & -1.53737600 \\
\hline $\mathrm{H}$ & -1.82057700 & -4.56594000 & 0.14768000 \\
\hline $\mathrm{H}$ & 0.77082400 & -3.63116200 & -1.02307600 \\
\hline $\mathrm{H}$ & 4.60606900 & -3.16696800 & -0.91332400 \\
\hline $\mathrm{H}$ & 7.65372500 & -0.27317900 & 2.29647900 \\
\hline $\mathrm{H}$ & 7.79795900 & -2.92642700 & 0.60190900 \\
\hline $\mathrm{H}$ & -1.19898000 & 1.95681900 & -0.72771500 \\
\hline $\mathrm{H}$ & -0.23383300 & 1.11859500 & -1.97436900 \\
\hline $\mathrm{H}$ & 4.61749300 & 1.57830600 & -1.50283700 \\
\hline $\mathrm{H}$ & 5.20032100 & 2.65497400 & -0.24261400 \\
\hline $\mathrm{H}$ & 4.95880300 & 4.65273100 & -3.09310200 \\
\hline $\mathrm{H}$ & -3.87265900 & 0.64664300 & 0.83153200 \\
\hline $\mathrm{H}$ & -6.36722000 & -2.09715900 & -1.37148900 \\
\hline $\mathrm{H}$ & -6.68258700 & 2.51188500 & 1.79483100 \\
\hline $\mathrm{H}$ & -8.54865800 & -1.62896700 & -1.3841650 \\
\hline $\mathrm{H}$ & -10.14973100 & 0.22567000 & 0.8005840 \\
\hline $\mathrm{H}$ & -10.48499800 & 1.98025700 & 0.92849700 \\
\hline $\mathrm{H}$ & -9.96557500 & 1.23896200 & -0.6157140 \\
\hline
\end{tabular}


Cartesian coordinates of the lowest-energy conformer of 3B (3B-1) at the B3LYP/6-31G(d,p) level in acetone (PCM).

\begin{tabular}{|c|c|c|c|}
\hline $\mathrm{C}$ & -2.51662600 & -1.00443100 & -1.13683200 \\
\hline 0 & -1.88598500 & 0.25433900 & -0.96585400 \\
\hline $\mathrm{C}$ & -0.54608700 & 0.17896800 & -0.45694300 \\
\hline $\mathrm{C}$ & 0.33218200 & -0.66702600 & -1.40819500 \\
\hline 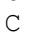 & -0.32139600 & -2.00079300 & -1.78585400 \\
\hline C & -1.77257600 & -1.78627700 & -2.21721200 \\
\hline 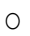 & -2.31745200 & -3.07936200 & -2.42467800 \\
\hline 0 & 0.44011100 & -2.57581400 & -2.83751500 \\
\hline $\mathrm{C}$ & 3.57055000 & 1.91714900 & 0.04207500 \\
\hline $\mathrm{C}$ & 4.26601400 & -0.60637700 & 0.20018800 \\
\hline $\mathrm{C}$ & 5.59401700 & -0.80779100 & 0.56234700 \\
\hline $\mathrm{C}$ & 6.04368700 & -1.83522800 & 1.37513200 \\
\hline $\mathrm{C}$ & 5.07290900 & -2.73025100 & 1.86511000 \\
\hline $\mathrm{C}$ & 3.73947700 & -2.57953900 & 1.49748500 \\
\hline $\mathrm{C}$ & 3.31837700 & -1.53751200 & 0.65207300 \\
\hline 0 & 7.33671500 & -2.05152300 & 1.73148900 \\
\hline 0 & 5.43959600 & -3.75819600 & 2.67418900 \\
\hline $\mathrm{C}$ & 2.12127000 & 1.62578300 & 0.42296600 \\
\hline $\mathrm{C}$ & 1.88203700 & -1.54384200 & 0.26904700 \\
\hline 0 & 1.01594700 & -2.13791100 & 0.88488600 \\
\hline 0 & 1.76846900 & 1.17903700 & 1.49421500 \\
\hline 0 & 1.67046200 & -0.84583200 & -0.87346300 \\
\hline 0 & -3.82015400 & -0.77904300 & -1.61532300 \\
\hline $\mathrm{C}$ & -0.12520200 & 1.65943900 & -0.37354100 \\
\hline 0 & 1.27933900 & 1.88982800 & -0.59519000 \\
\hline $\mathrm{C}$ & 4.23047100 & 0.65597000 & -0.63999600 \\
\hline $\mathrm{C}$ & 5.73372400 & 0.93892500 & -0.83093300 \\
\hline 0 & 6.46765400 & 0.10418400 & -0.01237400 \\
\hline 0 & 6.27962100 & 1.74952300 & -1.53107800 \\
\hline $\mathrm{C}$ & 3.68680800 & 3.16876300 & -0.84192000 \\
\hline $\mathrm{C}$ & 3.09166200 & 4.38945900 & -0.17890700 \\
\hline 0 & 2.54065600 & 4.40514600 & 0.90496300 \\
\hline 0 & 3.24485800 & 5.48812700 & -0.94058700 \\
\hline $\mathrm{H}$ & 3.77057000 & 0.50434100 & -1.62018700 \\
\hline $\mathrm{H}$ & 4.08083000 & 2.08939400 & 0.99353600 \\
\hline $\mathrm{C}$ & -4.81419900 & -0.42030300 & -0.74312000 \\
\hline $\mathrm{C}$ & -4.58425300 & 0.02223800 & 0.55425800 \\
\hline $\mathrm{C}$ & -5.68082600 & 0.35583700 & 1.35860400 \\
\hline $\mathrm{C}$ & -7.02929700 & 0.26258000 & 0.87887600 \\
\hline $\mathrm{C}$ & -7.19807600 & -0.18843600 & -0.46365500 \\
\hline $\mathrm{C}$ & -6.11165700 & -0.52380500 & -1.26076700 \\
\hline 0 & -5.41738500 & 0.77920800 & 2.59930000 \\
\hline 0 & -8.45965500 & -0.29364400 & -0.95180400 \\
\hline $\mathrm{C}$ & -8.12202200 & 0.63482700 & 1.78117500 \\
\hline 0 & -7.86758400 & 1.02788800 & 2.94327100 \\
\hline $\mathrm{C}$ & -9.57548600 & 0.56913300 & 1.37983500 \\
\hline $\mathrm{H}$ & -2.54987600 & -1.56093400 & -0.18856400 \\
\hline $\mathrm{H}$ & -0.54826100 & -0.28449300 & 0.53429900 \\
\hline $\mathrm{H}$ & 0.51031500 & -0.10654700 & -2.32965500 \\
\hline $\mathrm{H}$ & -0.33307800 & -2.66048000 & -0.91139800 \\
\hline $\mathrm{H}$ & -1.79987200 & -1.18575500 & -3.13766300 \\
\hline $\mathrm{H}$ & -3.18242800 & -2.97118100 & -2.84428900 \\
\hline $\mathrm{H}$ & -0.03553500 & -3.37770200 & -3.10138500 \\
\hline $\mathrm{H}$ & 3.01092200 & -3.29510900 & 1.85867400 \\
\hline $\mathrm{H}$ & 7.91431200 & -1.38324600 & 1.33358100 \\
\hline $\mathrm{H}$ & 6.39872000 & -3.71501600 & 2.81460300 \\
\hline $\mathrm{H}$ & -0.41954500 & 2.08685800 & 0.58613100 \\
\hline $\mathrm{H}$ & -0.61846600 & 2.19488000 & -1.18514000 \\
\hline $\mathrm{H}$ & 3.18045200 & 3.02174800 & -1.80091300 \\
\hline $\mathrm{H}$ & 4.73136300 & 3.38296800 & -1.07739200 \\
\hline $\mathrm{H}$ & 2.84161100 & 6.23387200 & -0.46233400 \\
\hline $\mathrm{H}$ & -3.58948600 & 0.15571100 & 0.95543800 \\
\hline $\mathrm{H}$ & -6.25256900 & -0.87809600 & -2.27644600 \\
\hline $\mathrm{H}$ & -6.31594700 & 0.97228200 & 3.01007700 \\
\hline $\mathrm{H}$ & -8.42588900 & -0.61036300 & -1.86604100 \\
\hline $\mathrm{H}$ & -9.77152600 & 1.20399100 & 0.51216600 \\
\hline $\mathrm{H}$ & -9.85567600 & -0.44675100 & 1.09015900 \\
\hline $\mathrm{H}$ & -10.17551800 & 0.89722500 & 2.22938600 \\
\hline
\end{tabular}


Cartesian coordinates of the lowest-energy conformer of 3C (3C-1) at the B3LYP/6-31G(d,p) level in acetone (PCM).

\begin{tabular}{|c|c|c|c|}
\hline C & -2.52882200 & -1.65077100 & 0.09139800 \\
\hline O & -1.92968400 & -0.65078300 & -0.72675700 \\
\hline C & -0.61030900 & -0.27367400 & -0.32702600 \\
\hline C & 0.30014800 & -1.50755300 & -0.45177000 \\
\hline $\mathrm{C}$ & -0.25735800 & -2.63946400 & 0.41190400 \\
\hline C & -1.71065900 & -2.93579000 & 0.00369200 \\
\hline 0 & -2.26976100 & -3.90830600 & 0.86315700 \\
\hline 0 & 0.50503600 & -3.83715200 & 0.37365500 \\
\hline C & 3.18857500 & 2.05454900 & -0.39279000 \\
\hline C & 4.29173300 & -0.15045200 & 0.45296500 \\
\hline C & 5.59757000 & 0.03287200 & 0.89969700 \\
\hline C & 6.64001800 & -0.85646700 & 0.69677800 \\
\hline C & 6.34281700 & -2.03110900 & -0.01993200 \\
\hline C & 5.04131500 & -2.27006700 & -0.44635600 \\
\hline C & 3.99684700 & -1.35508000 & -0.20906100 \\
\hline 0 & 7.91311900 & -0.68907000 & 1.13534400 \\
\hline 0 & 7.31460300 & -2.94709900 & -0.26570300 \\
\hline C & 2.11408300 & 1.37901900 & -1.23801300 \\
\hline C & 2.65065600 & -1.81772800 & -0.62497200 \\
\hline 0 & 2.46344300 & -2.75113400 & -1.39061800 \\
\hline 0 & 2.32605100 & 0.70566600 & -2.22255500 \\
\hline 0 & 1.63668000 & -1.14929800 & -0.02334600 \\
\hline 0 & -3.80781500 & -1.90824800 & -0.42362900 \\
\hline C & -0.23443200 & 0.89046100 & -1.24539100 \\
\hline 0 & 0.89591100 & 1.58770300 & -0.69364100 \\
\hline C & 3.53337600 & 1.11154500 & 0.82748400 \\
\hline C & 4.54944000 & 1.82004300 & 1.74208000 \\
\hline 0 & 5.77517200 & 1.19501000 & 1.63553400 \\
\hline 0 & 4.40359100 & 2.77898600 & 2.45110400 \\
\hline C & 2.75786100 & 3.46405300 & 0.04715500 \\
\hline $\mathrm{C}$ & 2.40317100 & 4.34372000 & -1.13033300 \\
\hline 0 & 2.38798700 & 3.99431000 & -2.29462200 \\
\hline 0 & 2.09953200 & 5.59331900 & -0.73416200 \\
\hline $\mathrm{H}$ & 2.61997500 & 0.92497700 & 1.39828400 \\
\hline $\mathrm{H}$ & 4.07274100 & 2.12286600 & -1.03050200 \\
\hline C & -4.83792500 & -1.05236300 & -0.14067300 \\
\hline C & -4.67356300 & 0.20669900 & 0.42637500 \\
\hline $\mathrm{C}$ & -5.80731400 & 0.98849900 & 0.67761700 \\
\hline $\mathrm{C}$ & -7.12921700 & 0.53315700 & 0.35808600 \\
\hline C & -7.23117600 & -0.76201500 & -0.23131300 \\
\hline C & -6.10798000 & -1.54077400 & -0.47504700 \\
\hline 0 & -5.60525600 & 2.19320400 & 1.22259000 \\
\hline 0 & -8.46609500 & -1.22808400 & -0.54600700 \\
\hline C & -8.26309300 & 1.41398000 & 0.64763300 \\
\hline 0 & -8.06613200 & 2.54055600 & 1.15990700 \\
\hline $\mathrm{C}$ & -9.69389300 & 1.03146600 & 0.35731900 \\
\hline $\mathrm{H}$ & -2.59676900 & -1.30313000 & 1.13310800 \\
\hline $\mathrm{H}$ & -0.60907800 & 0.08369600 & 0.71348500 \\
\hline $\mathrm{H}$ & 0.34287600 & -1.81410000 & -1.50043800 \\
\hline $\mathrm{H}$ & -0.25957600 & -2.31362700 & 1.46067300 \\
\hline $\mathrm{H}$ & -1.73007800 & -3.26857100 & -1.04613100 \\
\hline $\mathrm{H}$ & -1.60703100 & -4.61487600 & 0.91985600 \\
\hline $\mathrm{H}$ & 0.96011100 & -3.88059400 & -0.48392400 \\
\hline $\mathrm{H}$ & 4.82132400 & -3.19822200 & -0.95888000 \\
\hline $\mathrm{H}$ & 7.99654300 & 0.14133300 & 1.62774900 \\
\hline $\mathrm{H}$ & 8.14673100 & -2.62760500 & 0.11770500 \\
\hline $\mathrm{H}$ & -1.05958200 & 1.60363900 & -1.26346700 \\
\hline $\mathrm{H}$ & -0.01173400 & 0.55334700 & -2.25879300 \\
\hline $\mathrm{H}$ & 3.55132000 & 3.96055700 & 0.60869400 \\
\hline $\mathrm{H}$ & 1.89216600 & 3.42422800 & 0.71483000 \\
\hline $\mathrm{H}$ & 1.87768200 & 6.10683800 & -1.53076200 \\
\hline $\mathrm{H}$ & -3.70155500 & 0.62443300 & 0.64755400 \\
\hline $\mathrm{H}$ & -6.19766900 & -2.53077700 & -0.90946700 \\
\hline $\mathrm{H}$ & -6.52222500 & 2.59796400 & 1.31780200 \\
\hline $\mathrm{H}$ & -8.38774000 & -2.11037800 & -0.93685700 \\
\hline $\mathrm{H}$ & -10.33404800 & 1.85761300 & 0.66895100 \\
\hline $\mathrm{H}$ & -9.83593400 & 0.82501200 & -0.70646000 \\
\hline $\mathrm{H}$ & -9.97071000 & 0.11821300 & 0.8899460 \\
\hline
\end{tabular}




\section{Cartesian coordinates of the lowest-energy conformer of 3D (3D-1) at the B3LYP/6-31G(d,p) level in acetone $(\mathrm{PCM})$.}

\begin{tabular}{|c|c|c|c|}
\hline C & -2.60878100 & -1.48455900 & -0.55291100 \\
\hline 0 & -1.99727600 & -0.34391500 & -1.13303500 \\
\hline $\mathrm{C}$ & -0.63581100 & -0.13239500 & -0.73271400 \\
\hline C & 0.21874000 & -1.36926700 & -1.09082600 \\
\hline $\mathrm{C}$ & -0.42324000 & -2.68419400 & -0.63500600 \\
\hline $\mathrm{C}$ & -1.89250500 & -2.73644900 & -1.05439600 \\
\hline 0 & -2.42013100 & -3.92666200 & -0.49176200 \\
\hline $\mathrm{O}$ & 0.31058500 & -3.75056600 & -1.21837600 \\
\hline C & 3.37406300 & 1.78007700 & -1.04378000 \\
\hline C & 4.22525400 & -0.47693100 & 0.00176100 \\
\hline C & 5.59571600 & -0.54492200 & 0.22918000 \\
\hline C & 6.17632200 & -1.18892600 & 1.30945900 \\
\hline $\mathrm{C}$ & 5.30199800 & -1.80001800 & 2.23118500 \\
\hline C & 3.92596200 & -1.77287400 & 2.01804800 \\
\hline $\mathrm{C}$ & 3.36993400 & -1.13618200 & 0.89481200 \\
\hline 0 & 7.50954400 & -1.28005900 & 1.55471900 \\
\hline 0 & 5.80268200 & -2.44083000 & 3.31939200 \\
\hline $\mathrm{C}$ & 1.90184400 & 1.64749500 & -0.67643900 \\
\hline C & 1.89986300 & -1.27380600 & 0.70046800 \\
\hline 0 & 1.10338700 & -1.45659500 & 1.60129800 \\
\hline 0 & 1.45289200 & 1.80257600 & 0.44189600 \\
\hline 0 & 1.58428500 & -1.22458500 & -0.61735700 \\
\hline 0 & -3.93437800 & -1.55321400 & -1.01741000 \\
\hline $\mathrm{C}$ & -0.25642200 & 1.13516700 & -1.51999900 \\
\hline 0 & 1.15801800 & 1.30911500 & -1.74207800 \\
\hline $\mathrm{C}$ & 4.03074200 & 0.36809800 & -1.23541400 \\
\hline $\mathrm{C}$ & 5.47765100 & 0.59094600 & -1.70195800 \\
\hline 0 & 6.35035700 & 0.07953500 & -0.75715600 \\
\hline 0 & 5.87634900 & 1.15145500 & -2.68597500 \\
\hline $\mathrm{C}$ & 4.09013700 & 2.64737800 & -0.00507500 \\
\hline $\mathrm{C}$ & 3.56944700 & 4.06893400 & -0.00978600 \\
\hline 0 & 2.74569700 & 4.51180300 & -0.78548000 \\
\hline 0 & 4.14503700 & 4.80748700 & 0.95595300 \\
\hline $\mathrm{H}$ & 3.48403400 & -0.15252600 & -2.02583200 \\
\hline $\mathrm{H}$ & 3.42304400 & 2.26690200 & -2.02147900 \\
\hline $\mathrm{C}$ & -4.89761600 & -0.75878600 & -0.45293600 \\
\hline C & -4.62282700 & 0.32748000 & 0.36950800 \\
\hline C & -5.69140000 & 1.06560000 & 0.89295600 \\
\hline $\mathrm{C}$ & -7.05607600 & 0.73911200 & 0.59619600 \\
\hline $\mathrm{C}$ & -7.27145500 & -0.38004800 & -0.26143200 \\
\hline $\mathrm{C}$ & -6.21286500 & -1.11728400 & -0.77527600 \\
\hline 0 & -5.38504200 & 2.10346100 & 1.67852300 \\
\hline 0 & -8.54964500 & -0.72082700 & -0.56315700 \\
\hline $\mathrm{C}$ & -8.11711900 & 1.56574600 & 1.17716500 \\
\hline 0 & -7.82238000 & 2.53510400 & 1.91412500 \\
\hline $\mathrm{C}$ & -9.58411800 & 1.30908100 & 0.93209100 \\
\hline $\mathrm{H}$ & -2.59086900 & -1.42417700 & 0.54529000 \\
\hline $\mathrm{H}$ & -0.58089900 & 0.04411100 & 0.34629900 \\
\hline $\mathrm{H}$ & 0.34351600 & -1.41749600 & -2.17624900 \\
\hline $\mathrm{H}$ & -0.39184700 & -2.74624800 & 0.45783200 \\
\hline $\mathrm{H}$ & -1.96480800 & -2.74236900 & -2.15113200 \\
\hline $\mathrm{H}$ & -3.30182700 & -4.06481900 & -0.86530200 \\
\hline $\mathrm{H}$ & -0.16165800 & -4.56122700 & -0.97595000 \\
\hline $\mathrm{H}$ & 3.27621500 & -2.27828900 & 2.72246800 \\
\hline $\mathrm{H}$ & 8.01443000 & -0.84159800 & 0.85380200 \\
\hline $\mathrm{H}$ & 6.77053700 & -2.37390100 & 3.30027700 \\
\hline $\mathrm{H}$ & -0.65931400 & 2.01758700 & -1.01946200 \\
\hline $\mathrm{H}$ & -0.67973600 & 1.05416100 & -2.52143200 \\
\hline $\mathrm{H}$ & 5.16662700 & 2.69569800 & -0.20121900 \\
\hline $\mathrm{H}$ & 3.96845300 & 2.23982500 & 1.00197600 \\
\hline $\mathrm{H}$ & 3.77937000 & 5.70741800 & 0.89359300 \\
\hline $\mathrm{H}$ & -3.61509700 & 0.64969300 & 0.59077000 \\
\hline $\mathrm{H}$ & -6.38848800 & -1.97545300 & -1.41528800 \\
\hline $\mathrm{H}$ & -6.26906300 & 2.50464500 & 1.94486000 \\
\hline $\mathrm{H}$ & -8.54763500 & -1.48690500 & -1.15510000 \\
\hline $\mathrm{H}$ & -9.81726700 & 1.35499900 & -0.13461000 \\
\hline $\mathrm{H}$ & -9.86803100 & 0.30881500 & 1.26879100 \\
\hline $\mathrm{H}$ & -10.15414400 & 2.06404800 & 1.4748650 \\
\hline
\end{tabular}


Cartesian coordinates of the lowest-energy conformer of $\mathbf{3 A}(\mathbf{3 A - 1})$ at the B3LYP/6-31G(d,p) level in $\mathrm{MeOH}(\mathrm{PCM})$.

\begin{tabular}{|c|c|c|c|}
\hline C & -2.71530100 & -1.49551800 & -0.12357800 \\
\hline O & -2.08978500 & -0.36507300 & -0.72160700 \\
\hline C & -0.77284400 & -0.08672200 & -0.23982500 \\
\hline C & 0.13228700 & -1.28410300 & -0.58983400 \\
\hline $\mathrm{C}$ & -0.45899700 & -2.54720400 & 0.04187100 \\
\hline C & -1.90520700 & -2.74785000 & -0.44240600 \\
\hline O & -2.48408400 & -3.86162800 & 0.20644800 \\
\hline O & 0.28841200 & -3.73253500 & -0.18768600 \\
\hline C & 3.09858500 & 2.10328900 & -0.03264900 \\
\hline C & 4.09635000 & -0.21913600 & 0.70207900 \\
\hline C & 5.34512200 & -0.15697500 & 1.31273300 \\
\hline $\mathrm{C}$ & 6.34633900 & -1.10151200 & 1.15426500 \\
\hline C & 6.06381100 & -2.19270600 & 0.30867200 \\
\hline C & 4.81498000 & -2.30211800 & -0.29496600 \\
\hline $\mathrm{C}$ & 3.80896800 & -1.33723100 & -0.09656500 \\
\hline O & 7.56849600 & -1.06122100 & 1.74138000 \\
\hline O & 7.00023300 & -3.15349200 & 0.10015000 \\
\hline C & 1.95491800 & 1.64393000 & -0.92282900 \\
\hline C & 2.48918800 & -1.64764000 & -0.69937200 \\
\hline O & 2.33092500 & -2.42540800 & -1.62728300 \\
\hline O & 2.08459000 & 1.18765000 & -2.04002100 \\
\hline O & 1.46061200 & -1.03289700 & -0.06553100 \\
\hline 0 & -3.98440200 & -1.63204000 & -0.70553200 \\
\hline C & -0.39699500 & 1.23774200 & -0.90564400 \\
\hline 0 & 0.77916100 & 1.78477700 & -0.28151100 \\
\hline C & 3.36442600 & 1.04493100 & 1.09320400 \\
\hline C & 4.33547200 & 1.68306400 & 2.09861600 \\
\hline O & 5.50803000 & 0.95149700 & 2.13378400 \\
\hline 0 & 4.19712000 & 2.68120300 & 2.75105900 \\
\hline C & 4.33469500 & 2.42752700 & -0.87334700 \\
\hline C & 4.10013900 & 3.62421400 & -1.77045000 \\
\hline O & 3.08694200 & 4.29459900 & -1.79513200 \\
\hline O & 5.16871300 & 3.87309600 & -2.54846700 \\
\hline $\mathrm{H}$ & 2.41633400 & 0.85207900 & 1.60280400 \\
\hline $\mathrm{H}$ & 2.75995700 & 3.00491400 & 0.48512100 \\
\hline C & -5.00981100 & -0.82440100 & -0.29259900 \\
\hline C & -4.84325300 & 0.29879500 & 0.51010700 \\
\hline C & -5.97192600 & 1.04169000 & 0.87594800 \\
\hline C & -7.29037600 & 0.68435800 & 0.43864400 \\
\hline C & -7.39417400 & -0.47025400 & -0.39295800 \\
\hline C & -6.27613800 & -1.21231800 & -0.74979700 \\
\hline 0 & -5.76832100 & 2.11443500 & 1.64872600 \\
\hline 0 & -8.62520200 & -0.83838000 & -0.82851600 \\
\hline C & -8.41907100 & 1.51517000 & 0.86420000 \\
\hline 0 & -8.22038700 & 2.51767400 & 1.58968000 \\
\hline C & -9.84666700 & 1.22429300 & 0.47051800 \\
\hline $\mathrm{H}$ & -2.80399600 & -1.35515100 & 0.96396300 \\
\hline $\mathrm{H}$ & -0.78102800 & 0.05469500 & 0.85112700 \\
\hline $\mathrm{H}$ & 0.19908200 & -1.38782100 & -1.67637300 \\
\hline $\mathrm{H}$ & -0.48345400 & -2.41131100 & 1.13145300 \\
\hline $\mathrm{H}$ & -1.90757600 & -2.87420400 & -1.53663600 \\
\hline $\mathrm{H}$ & -1.82015800 & -4.56716600 & 0.14772100 \\
\hline $\mathrm{H}$ & 0.76820500 & -3.63309000 & -1.02669100 \\
\hline $\mathrm{H}$ & 4.60705800 & -3.16637800 & -0.91373700 \\
\hline $\mathrm{H}$ & 7.65308100 & -0.27218200 & 2.29750500 \\
\hline $\mathrm{H}$ & 7.79788800 & -2.92530300 & 0.60354300 \\
\hline $\mathrm{H}$ & -1.19944900 & 1.95548000 & -0.73151300 \\
\hline $\mathrm{H}$ & -0.23368800 & 1.11654700 & -1.97725600 \\
\hline $\mathrm{H}$ & 4.61900500 & 1.57901500 & -1.50150900 \\
\hline $\mathrm{H}$ & 5.19921800 & 2.65624300 & -0.24086900 \\
\hline $\mathrm{H}$ & 4.96128100 & 4.65429600 & -3.09088500 \\
\hline $\mathrm{H}$ & -3.87202800 & 0.64492500 & 0.83434100 \\
\hline $\mathrm{H}$ & -6.36727200 & -2.09748300 & -1.37006300 \\
\hline $\mathrm{H}$ & -6.68171500 & 2.51203300 & 1.79557200 \\
\hline $\mathrm{H}$ & -8.54907200 & -1.62802300 & -1.38369800 \\
\hline $\mathrm{H}$ & -10.14923100 & 0.22742600 & 0.80044800 \\
\hline $\mathrm{H}$ & -10.48393000 & 1.98210000 & 0.92783300 \\
\hline $\mathrm{H}$ & -9.96406700 & 1.24041900 & -0.61598100 \\
\hline
\end{tabular}


Cartesian coordinates of the lowest-energy conformer of 3B (3B-1) at the B3LYP/6-31G(d,p) level in $\mathrm{MeOH}(\mathrm{PCM})$.

\begin{tabular}{|c|c|c|c|}
\hline $\mathrm{C}$ & -2.51733000 & -1.00805200 & -1.13264700 \\
\hline 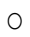 & -1.88603100 & 0.25095700 & -0.96495500 \\
\hline $\mathrm{C}$ & -0.54625800 & 0.17678300 & -0.45562500 \\
\hline $\mathrm{C}$ & 0.33173900 & -0.67205200 & -1.40450800 \\
\hline 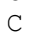 & -0.32202600 & -2.00718300 & -1.77671100 \\
\hline$C$ & -1.77287900 & -1.79398500 & -2.20982100 \\
\hline 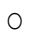 & -2.31776400 & -3.08783800 & -2.41246700 \\
\hline 0 & 0.43974100 & -2.58746600 & -2.82529200 \\
\hline $\mathrm{C}$ & 3.57028300 & 1.91783000 & 0.03999500 \\
\hline $\mathrm{C}$ & 4.26742100 & -0.60470100 & 0.19904300 \\
\hline $\mathrm{C}$ & 5.59633400 & -0.80505700 & 0.55856200 \\
\hline $\mathrm{C}$ & 6.04812200 & -1.83082600 & 1.37235100 \\
\hline $\mathrm{C}$ & 5.07850900 & -2.72519000 & 1.86604400 \\
\hline $\mathrm{C}$ & 3.74422400 & -2.57554200 & 1.50114800 \\
\hline $\mathrm{C}$ & 3.32094800 & -1.53516500 & 0.65471900 \\
\hline 0 & 7.34167200 & -2.04632800 & 1.72646600 \\
\hline 0 & 5.44719400 & -3.75155500 & 2.67626900 \\
\hline $\mathrm{C}$ & 2.12110600 & 1.62645100 & 0.42118700 \\
\hline $\mathrm{C}$ & 1.88392000 & -1.54260600 & 0.27433700 \\
\hline 0 & 1.01875400 & -2.13413200 & 0.89415600 \\
\hline 0 & 1.76835300 & 1.18181600 & 1.49354900 \\
\hline 0 & 1.67059200 & -0.84900600 & -0.87027300 \\
\hline 0 & -3.82021800 & -0.78291600 & -1.61290000 \\
\hline $\mathrm{C}$ & -0.12527700 & 1.65742000 & -0.37619100 \\
\hline 0 & 1.27945400 & 1.88732500 & -0.59757400 \\
\hline $\mathrm{C}$ & 4.22946900 & 0.65660500 & -0.64266900 \\
\hline $\mathrm{C}$ & 5.73213300 & 0.93963600 & -0.83748400 \\
\hline 0 & 6.46820200 & 0.10632600 & -0.01967900 \\
\hline 0 & 6.27615400 & 1.74926900 & -1.54030100 \\
\hline $\mathrm{C}$ & 3.68590600 & 3.16972600 & -0.84374500 \\
\hline $\mathrm{C}$ & 3.08997200 & 4.38995600 & -0.18055400 \\
\hline 0 & 2.53632400 & 4.40434700 & 0.90212500 \\
\hline 0 & 3.24608200 & 5.48945900 & -0.94001900 \\
\hline $\mathrm{H}$ & 3.76736700 & 0.50382900 & -1.62162600 \\
\hline $\mathrm{H}$ & 4.08120100 & 2.08989800 & 0.99113000 \\
\hline $\mathrm{C}$ & -4.81505300 & -0.42235500 & -0.74249700 \\
\hline $\mathrm{C}$ & -4.58625300 & 0.02262500 & 0.55441600 \\
\hline $\mathrm{C}$ & -5.68349500 & 0.35811400 & 1.35686200 \\
\hline $\mathrm{C}$ & -7.03150500 & 0.26456300 & 0.87591300 \\
\hline $\mathrm{C}$ & -7.19912400 & -0.18871800 & -0.46612000 \\
\hline $\mathrm{C}$ & -6.11191400 & -0.52621700 & -1.26134900 \\
\hline 0 & -5.42139100 & 0.78380700 & 2.59727700 \\
\hline 0 & -8.46001300 & -0.29382100 & -0.95541200 \\
\hline $\mathrm{C}$ & -8.12500600 & 0.63864300 & 1.77627800 \\
\hline 0 & -7.87166500 & 1.03409500 & 2.93798900 \\
\hline $\mathrm{C}$ & -9.57803500 & 0.57206000 & 1.37379100 \\
\hline $\mathrm{H}$ & -2.55178500 & -1.56157500 & -0.18270700 \\
\hline $\mathrm{H}$ & -0.54876500 & -0.28404600 & 0.53684700 \\
\hline $\mathrm{H}$ & 0.50916200 & -0.11460600 & -2.32792300 \\
\hline $\mathrm{H}$ & -0.33451400 & -2.66298500 & -0.89928900 \\
\hline $\mathrm{H}$ & -1.79954500 & -1.19711400 & -3.13258800 \\
\hline $\mathrm{H}$ & -3.17982600 & -2.98183000 & -2.83857900 \\
\hline $\mathrm{H}$ & -0.03844500 & -3.38817900 & -3.08831800 \\
\hline $\mathrm{H}$ & 3.01683600 & -3.29093700 & 1.86506100 \\
\hline $\mathrm{H}$ & 7.91874800 & -1.37885700 & 1.32638500 \\
\hline $\mathrm{H}$ & 6.40677600 & -3.70858500 & 2.81383100 \\
\hline $\mathrm{H}$ & -0.42024300 & 2.08761200 & 0.58205100 \\
\hline $\mathrm{H}$ & -0.61787900 & 2.19058900 & -1.18964500 \\
\hline $\mathrm{H}$ & 3.17963800 & 3.02260100 & -1.80277900 \\
\hline $\mathrm{H}$ & 4.73034200 & 3.38457900 & -1.07899500 \\
\hline $\mathrm{H}$ & 2.84210900 & 6.23513400 & -0.46222100 \\
\hline $\mathrm{H}$ & -3.59177700 & 0.15633100 & 0.95627700 \\
\hline $\mathrm{H}$ & -6.25199500 & -0.88251900 & -2.27641500 \\
\hline $\mathrm{H}$ & -6.32061000 & 0.97802600 & 3.00629100 \\
\hline $\mathrm{H}$ & -8.42570700 & -0.61131100 & -1.86941300 \\
\hline $\mathrm{H}$ & -9.77343000 & 1.20576400 & 0.50513100 \\
\hline $\mathrm{H}$ & -9.85774200 & -0.44424900 & 1.08516800 \\
\hline $\mathrm{H}$ & -10.17900600 & 0.90113400 & 2.2223000 \\
\hline
\end{tabular}


Cartesian coordinates of the lowest-energy conformer of 3C (3C-1) at the B3LYP/6-31G(d,p) level in $\mathrm{MeOH}(\mathrm{PCM})$.

\begin{tabular}{|c|c|c|c|}
\hline $\mathrm{C}$ & 2.52792400 & -1.65061000 & -0.09346000 \\
\hline 0 & 1.92969200 & -0.65106000 & 0.72581800 \\
\hline $\mathrm{C}$ & 0.61014300 & -0.27341100 & 0.32656600 \\
\hline $\mathrm{C}$ & -0.30028300 & -1.50717300 & 0.45195400 \\
\hline $\mathrm{C}$ & 0.25593700 & -2.63912600 & -0.41244300 \\
\hline $\mathrm{C}$ & 1.70964700 & -2.93562200 & -0.00586000 \\
\hline 0 & 2.26819300 & -3.90781600 & -0.86626300 \\
\hline 0 & -0.50722300 & -3.83643100 & -0.37316800 \\
\hline $\mathrm{C}$ & -3.18855500 & 2.05505600 & 0.39340900 \\
\hline $\mathrm{C}$ & -4.29192300 & -0.15019500 & -0.45205800 \\
\hline $\mathrm{C}$ & -5.59743000 & 0.03230900 & -0.90016900 \\
\hline $\mathrm{C}$ & -6.63963200 & -0.85751900 & -0.69776200 \\
\hline $\mathrm{C}$ & -6.34226900 & -2.03160100 & 0.01997700 \\
\hline $\mathrm{C}$ & -5.04113600 & -2.26962100 & 0.44790700 \\
\hline $\mathrm{C}$ & -3.99689800 & -1.35422300 & 0.21094700 \\
\hline 0 & -7.91231900 & -0.69162700 & -1.13733800 \\
\hline 0 & -7.31381700 & -2.94800200 & 0.26544400 \\
\hline $\mathrm{C}$ & -2.11377600 & 1.38008300 & 1.23863400 \\
\hline $\mathrm{C}$ & -2.65079900 & -1.81571000 & 0.62828400 \\
\hline 0 & -2.46358000 & -2.74731700 & 1.39626100 \\
\hline 0 & -2.32562800 & 0.70824900 & 2.22454700 \\
\hline 0 & -1.63713200 & -1.14889400 & 0.02474500 \\
\hline 0 & 3.80725500 & -1.90906200 & 0.42089400 \\
\hline $\mathrm{C}$ & 0.23469900 & 0.89069500 & 1.24512300 \\
\hline 0 & -0.89606900 & 1.58764200 & 0.69371900 \\
\hline $\mathrm{C}$ & -3.53352900 & 1.11173500 & -0.82664400 \\
\hline $\mathrm{C}$ & -4.54934200 & 1.81972500 & -1.74188600 \\
\hline 0 & -5.77459500 & 1.19402500 & -1.63668300 \\
\hline 0 & -4.40341500 & 2.77872000 & -2.45101000 \\
\hline $\mathrm{C}$ & -2.75802000 & 3.46445100 & -0.04708800 \\
\hline $\mathrm{C}$ & -2.40178100 & 4.34435600 & 1.12974700 \\
\hline 0 & -2.38282900 & 3.99433900 & 2.29396300 \\
\hline 0 & -2.10144300 & 5.59440500 & 0.73340900 \\
\hline $\mathrm{H}$ & -2.62011500 & 0.92480100 & -1.39731400 \\
\hline $\mathrm{H}$ & -4.07267100 & 2.12361300 & 1.03115700 \\
\hline $\mathrm{C}$ & 4.83763300 & -1.05319800 & 0.13870000 \\
\hline $\mathrm{C}$ & 4.67351800 & 0.20630700 & -0.42767300 \\
\hline $\mathrm{C}$ & 5.80748500 & 0.98804500 & -0.67774700 \\
\hline $\mathrm{C}$ & 7.12923100 & 0.53244800 & -0.35788000 \\
\hline $\mathrm{C}$ & 7.23093100 & -0.76326500 & 0.23064800 \\
\hline $\mathrm{C}$ & 6.10740100 & -1.54203200 & 0.47330400 \\
\hline 0 & 5.60594800 & 2.19331500 & -1.22207500 \\
\hline 0 & 8.46545500 & -1.22946500 & 0.54545500 \\
\hline $\mathrm{C}$ & 8.26330100 & 1.41330500 & -0.64607700 \\
\hline 0 & 8.06667300 & 2.54044700 & -1.15764100 \\
\hline $\mathrm{C}$ & 9.69388200 & 1.03052900 & -0.35549000 \\
\hline $\mathrm{H}$ & 2.59553600 & -1.30216800 & -1.13484200 \\
\hline $\mathrm{H}$ & 0.60867300 & 0.08397100 & -0.71382200 \\
\hline $\mathrm{H}$ & -0.34207900 & -1.81395600 & 1.50057100 \\
\hline $\mathrm{H}$ & 0.25657100 & -2.31362800 & -1.46120800 \\
\hline $\mathrm{H}$ & 1.72996300 & -3.26895700 & 1.04372800 \\
\hline $\mathrm{H}$ & 1.60598100 & -4.61496500 & -0.92182700 \\
\hline $\mathrm{H}$ & -0.95643700 & -3.88245600 & 0.48728100 \\
\hline $\mathrm{H}$ & -4.82117800 & -3.19747200 & 0.96101800 \\
\hline $\mathrm{H}$ & -7.99642200 & 0.13785200 & -1.63121400 \\
\hline $\mathrm{H}$ & -8.14560800 & -2.62961200 & -0.11970300 \\
\hline $\mathrm{H}$ & 1.05962500 & 1.60412400 & 1.26261700 \\
\hline $\mathrm{H}$ & 0.01249100 & 0.55373600 & 2.25868200 \\
\hline $\mathrm{H}$ & -3.55220300 & 3.96088300 & -0.60759900 \\
\hline $\mathrm{H}$ & -1.89302600 & 3.42437100 & -0.71568000 \\
\hline $\mathrm{H}$ & -1.87841800 & 6.10843800 & 1.52937600 \\
\hline $\mathrm{H}$ & 3.70160300 & 0.62396300 & -0.64949400 \\
\hline $\mathrm{H}$ & 6.19698700 & -2.53224300 & 0.90724400 \\
\hline $\mathrm{H}$ & 6.52319500 & 2.59781400 & -1.31642300 \\
\hline $\mathrm{H}$ & 8.38737000 & -2.11205300 & 0.93582500 \\
\hline $\mathrm{H}$ & 10.33432400 & 1.85685300 & -0.66607800 \\
\hline $\mathrm{H}$ & 9.83546300 & 0.82314400 & 0.70816200 \\
\hline $\mathrm{H}$ & 9.97089200 & 0.11776200 & -0.8888430 \\
\hline
\end{tabular}


Cartesian coordinates of the lowest-energy conformer of 3D (3D-1) at the B3LYP/6-31G(d,p) level in $\mathrm{MeOH}(\mathrm{PCM})$.

\begin{tabular}{|c|c|c|c|}
\hline $\mathrm{C}$ & -2.60918500 & -1.48424100 & -0.55180300 \\
\hline 0 & -1.99737600 & -0.34309600 & -1.13091300 \\
\hline $\mathrm{C}$ & -0.63602300 & -0.13163000 & -0.73026300 \\
\hline $\mathrm{C}$ & 0.21852200 & -1.36825000 & -1.08908100 \\
\hline C & -0.42331300 & -2.68321900 & -0.63331900 \\
\hline $\mathrm{C}$ & -1.89241000 & -2.73593500 & -1.05327900 \\
\hline 0 & -2.41980200 & -3.92625400 & -0.49075700 \\
\hline 0 & 0.31093300 & -3.74968100 & -1.21604100 \\
\hline $\mathrm{C}$ & 3.37420000 & 1.78087600 & -1.04339400 \\
\hline $\mathrm{C}$ & 4.22612200 & -0.47660400 & 0.00096800 \\
\hline $\mathrm{C}$ & 5.59679600 & -0.54533900 & 0.22697900 \\
\hline $\mathrm{C}$ & 6.17822300 & -1.18988600 & 1.30653100 \\
\hline $\mathrm{C}$ & 5.30427200 & -1.80087700 & 2.22884400 \\
\hline C & 3.92808300 & -1.77309900 & 2.01700900 \\
\hline $\mathrm{C}$ & 3.37124600 & -1.13576900 & 0.89446800 \\
\hline 0 & 7.51127600 & -1.28200600 & 1.55082500 \\
\hline 0 & 5.80561500 & -2.44221500 & 3.31642600 \\
\hline $\mathrm{C}$ & 1.90241300 & 1.64807500 & -0.67469700 \\
\hline $\mathrm{C}$ & 1.90106900 & -1.27272700 & 0.70127300 \\
\hline 0 & 1.10521900 & -1.45501800 & 1.60295100 \\
\hline 0 & 1.45466900 & 1.80209600 & 0.44442200 \\
\hline 0 & 1.58446300 & -1.22389800 & -0.61617400 \\
\hline 0 & -3.93433700 & -1.55245400 & -1.01748100 \\
\hline $\mathrm{C}$ & -0.25686200 & 1.13644500 & -1.51684100 \\
\hline 0 & 1.15750400 & 1.31075800 & -1.73966700 \\
\hline C & 4.03067100 & 0.36882900 & -1.23573000 \\
\hline $\mathrm{C}$ & 5.47716700 & 0.59134800 & -1.70344000 \\
\hline 0 & 6.35051600 & 0.07918100 & -0.76010900 \\
\hline 0 & 5.87499400 & 1.15238900 & -2.68767300 \\
\hline $\mathrm{C}$ & 4.09144800 & 2.64821500 & -0.00561700 \\
\hline $\mathrm{C}$ & 3.56937800 & 4.06924900 & -0.00819200 \\
\hline 0 & 2.74315700 & 4.51156600 & -0.78177300 \\
\hline 0 & 4.14605500 & 4.80763600 & 0.95667900 \\
\hline H & 3.48332800 & -0.15163400 & -2.02582700 \\
\hline $\mathrm{H}$ & 3.42224700 & 2.26749500 & -2.02123100 \\
\hline $\mathrm{C}$ & -4.89817400 & -0.75883700 & -0.45312100 \\
\hline $\mathrm{C}$ & -4.62422000 & 0.32686800 & 0.37060900 \\
\hline $\mathrm{C}$ & -5.69331600 & 1.06427100 & 0.89361000 \\
\hline $\mathrm{C}$ & -7.05772600 & 0.73806100 & 0.59525400 \\
\hline C & -7.27226300 & -0.38050700 & -0.26358400 \\
\hline $\mathrm{C}$ & -6.21299300 & -1.11718400 & -0.77700700 \\
\hline 0 & -5.38788700 & 2.10160200 & 1.68067000 \\
\hline 0 & -8.54986600 & -0.72095100 & -0.56677800 \\
\hline C & -8.11934000 & 1.56405300 & 1.17566200 \\
\hline 0 & -7.82540600 & 2.53304900 & 1.91376300 \\
\hline $\mathrm{C}$ & -9.58604500 & 1.30766700 & 0.92899500 \\
\hline H & -2.59209300 & -1.42442000 & 0.54641500 \\
\hline $\mathrm{H}$ & -0.58134400 & 0.04425100 & 0.34884500 \\
\hline $\mathrm{H}$ & 0.34292700 & -1.41606300 & -2.17453500 \\
\hline $\mathrm{H}$ & -0.39233300 & -2.74505300 & 0.45955900 \\
\hline $\mathrm{H}$ & -1.96435500 & -2.74188100 & -2.14999300 \\
\hline H & -3.29900400 & -4.06808400 & -0.86879800 \\
\hline $\mathrm{H}$ & -0.16290000 & -4.56009200 & -0.97573500 \\
\hline $\mathrm{H}$ & 3.27890500 & -2.27870200 & 2.72183000 \\
\hline $\mathrm{H}$ & 8.01647100 & -0.84316000 & 0.85034500 \\
\hline $\mathrm{H}$ & 6.77353400 & -2.37592200 & 3.29645200 \\
\hline $\mathrm{H}$ & -0.65951800 & 2.01861100 & -1.01565400 \\
\hline $\mathrm{H}$ & -0.68055000 & 1.05605200 & -2.51814400 \\
\hline $\mathrm{H}$ & 5.16743800 & 2.69785300 & -0.20413200 \\
\hline $\mathrm{H}$ & 3.97272300 & 2.23979600 & 1.00145600 \\
\hline $\mathrm{H}$ & 3.78012100 & 5.70757300 & 0.89560800 \\
\hline $\mathrm{H}$ & -3.61666000 & 0.64872800 & 0.59320600 \\
\hline $\mathrm{H}$ & -6.38796400 & -1.97489300 & -1.41778600 \\
\hline H & -6.27244700 & 2.50206600 & 1.94654200 \\
\hline $\mathrm{H}$ & -8.54767800 & -1.48698100 & -1.15887200 \\
\hline $\mathrm{H}$ & -9.81812100 & 1.35429800 & -0.13791100 \\
\hline $\mathrm{H}$ & -9.87033200 & 0.30718000 & 1.26472500 \\
\hline $\mathrm{H}$ & -10.15664400 & 2.06226500 & 1.47166700 \\
\hline
\end{tabular}


Cartesian coordinates of the lowest-energy conformer of $\mathbf{4 A}(\mathbf{4 A - 1})$ at the B3LYP/6-31G(d,p) level in $\mathrm{MeOH}(\mathrm{PCM})$.

\begin{tabular}{|c|c|c|}
\hline-0.60417200 & 2808100 & -0.10796100 \\
\hline 0.13182400 & 2.80341200 & 0.68733500 \\
\hline 1.63198100 & 2.76613800 & 0.38345500 \\
\hline 2.15471100 & 1.34984900 & 0.60986500 \\
\hline 1.42267700 & 0.42969300 & -0.18990700 \\
\hline 0.03751800 & 0.35169600 & 0.14868300 \\
\hline 3.49010700 & 1.29056300 & 0.16414700 \\
\hline 4.30734000 & 0.35168200 & 0.75418400 \\
\hline 5.64088800 & 0.39446000 & 0.15303400 \\
\hline 3.92796600 & -0.38310200 & 1.64864200 \\
\hline 6.60152500 & -0.45616100 & 0.57708500 \\
\hline 7.97195800 & -0.56796700 & 0.10197600 \\
\hline 2.33358200 & 3.65593800 & 30300 \\
\hline-0.30688000 & 4.11625500 & 65600 \\
\hline-1.97431400 & 1.72722100 & 0.35446600 \\
\hline-0.51383400 & -0.78026500 & -0.71300400 \\
\hline-1.85704400 & -1.05561800 & -0.26341700 \\
\hline-2.56145400 & -1.92964300 & -1.01802800 \\
\hline-2.08599200 & -2.50276600 & -1.98029500 \\
\hline-3.93910400 & -2.14564600 & -0.50234700 \\
\hline-2.94624300 & 1.47254200 & -0.54963600 \\
\hline-2 . & 1.386 & -1 \\
\hline-4.2 & 1. & \\
\hline-4.57682400 & -3.32682200 & -0.92383500 \\
\hline-5.82788500 & -3.69702400 & -0.44071500 \\
\hline-6.48577800 & -2.88715900 & 0.50584700 \\
\hline-5.84366500 & -1.71982300 & 0.88491100 \\
\hline-4.60621700 & -1.30329700 & 0.40164200 \\
\hline-6.39297400 & -0.85855900 & 1.82676700 \\
\hline 063100 & 0.187 & 225800 \\
\hline-4 . & 0. & 1. \\
\hline-5 . & 1 . & -0 \\
\hline-5 & 1. & \\
\hline-7.68899800 & -3.312 & 03500 \\
\hline-6.40205600 & -4.85233700 & -0.8654500 \\
\hline 8.80336800 & -1.54917100 & 0.68619800 \\
\hline 10.11661100 & -1.70493600 & 0.27090500 \\
\hline 10.63884900 & -0.87838900 & -0.74420500 \\
\hline 9.82619900 & 0.09621400 & -1.32723000 \\
\hline 8.5 & 0.25034400 & 53300 \\
\hline 11. & -1 & -1 \\
\hline 11. & -2 . & \\
\hline-5.54740900 & 2.748 & 07900 \\
\hline-4.93969300 & 3.738 & -1.14219700 \\
\hline-6.43600100 & 2.75107300 & -2.50711800 \\
\hline-0.58586100 & 1.96201300 & -1.17473400 \\
\hline-0.00527200 & 2.60339400 & 1.7603990 \\
\hline 1.7836030 & 3.01721200 & -0.6775350 \\
\hline 2.0985710 & 1.06639600 & 1.66754100 \\
\hline-0 . & 0.0 & 1 . \\
\hline & 1 . & -0 . \\
\hline 6.31 & -1 & 5200 \\
\hline 1.84700600 & 4.49381000 & 1.19381700 \\
\hline-1.17544900 & 4.25043800 & 0.77148300 \\
\hline 0.10487400 & -1.67189900 & -0.58471900 \\
\hline-0.52541400 & -0.50730200 & -1.7703650 \\
\hline-4.372598 & 2.15760800 & 0.841311 \\
\hline-4 & -3.97712900 & -1.62 \\
\hline-3 & 0. & 1 \\
\hline-6 . & 1. & -0.38 \\
\hline-5 . & 0.6372 & -1.6589220 \\
\hline-8.042163 & -2.68817900 & 1.6206260 \\
\hline 5805700 & -4.95538100 & -0.4201490 \\
\hline 09304 & -2.19085000 & 99540 \\
\hline 10.2449660 & 0.72364000 & -2.10690000 \\
\hline 962620 & 1.01342400 & -1.37750900 \\
\hline 216430 & -1.74670500 & -0.63960800 \\
\hline 816770 & -3.16349500 & 1.4558210 \\
\hline & 3.65877900 & -2.8 \\
\hline
\end{tabular}


Cartesian coordinates of the lowest-energy conformer of 4B (4B-1) at the B3LYP/6-31G(d,p) level in $\mathrm{MeOH}(\mathrm{PCM})$.

\begin{tabular}{|c|c|c|c|}
\hline C & 0.70746400 & 1.58943200 & 0.40606300 \\
\hline C & 0.06408100 & 2.48256500 & -0.64659400 \\
\hline $\mathrm{C}$ & -1.45379200 & 2.49358000 & -0.44747700 \\
\hline C & -1.97430900 & 1.05424800 & -0.40728000 \\
\hline 0 & -1.30266900 & 0.30264400 & 0.59904400 \\
\hline$u$ & 0.09996800 & 0.18359400 & 0.35650200 \\
\hline 0 & -3.33408900 & 1.08891900 & -0.03822800 \\
\hline $\mathrm{C}$ & -4.14310800 & 0.08235600 & -0.51717900 \\
\hline C & -5.51238600 & 0.26442500 & -0.03361100 \\
\hline 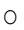 & -3.73246200 & -0.80482000 & -1.24390500 \\
\hline C & -6.48177700 & -0.60290200 & -0.39965100 \\
\hline c & -7.88849300 & -0.58077200 & -0.02912500 \\
\hline 0 & -2.09861600 & 3.17521500 & -1.50763000 \\
\hline 0 & 0.50877300 & 3.82695500 & -0.56323300 \\
\hline 0 & 2.11257900 & 1.50835200 & 0.09949000 \\
\hline C & 0.65022600 & -0.76727900 & 1.42548100 \\
\hline 0 & 2.06346500 & -1.01544400 & 1.22496300 \\
\hline $\mathrm{C}$ & 2.38331600 & -1.90162000 & 0.25449200 \\
\hline 0 & 1.54383000 & -2.52244500 & -0.37338500 \\
\hline $\mathrm{C}$ & 3.85062200 & -2.07298200 & 0.06306600 \\
\hline $\mathrm{C}$ & 2.97839300 & 1.62617700 & 1.12359200 \\
\hline 0 & 2.66208000 & 1.85116200 & 2.27260800 \\
\hline C & 4.41996200 & 1.41195800 & 0.69510000 \\
\hline $\mathrm{C}$ & 4.21408600 & -3.22938900 & -0.65425600 \\
\hline $\mathrm{C}$ & 5.54093300 & -3.58142600 & -0.87237900 \\
\hline $\mathrm{C}$ & 6.57088900 & -2.77165000 & -0.36015300 \\
\hline C & 6.18703200 & -1.63005900 & 0.32382700 \\
\hline $\mathrm{C}$ & 4.87000400 & -1.22765300 & 0.54317500 \\
\hline 0 & 7.13653600 & -0.78534500 & 0.88309000 \\
\hline $\mathrm{C}$ & 6.47192900 & 0.24023600 & 1.51613000 \\
\hline $\mathrm{C}$ & 4.95448800 & 0.07606900 & 1.31977600 \\
\hline C & 4.68052300 & 1.53605500 & -0.80841400 \\
\hline 0 & 7.06248000 & 1.11718800 & 2.08659400 \\
\hline 0 & 7.85137600 & -3.16753500 & -0.57231000 \\
\hline 0 & 5.83734600 & -4.71263700 & -1.56260300 \\
\hline C & -8.73447500 & -1.58194400 & -0.55585300 \\
\hline C & -10.08483300 & -1.60613000 & -0.24327100 \\
\hline $\mathrm{C}$ & -10.62953200 & -0.62765800 & 0.61232000 \\
\hline $\mathrm{C}$ & -9.80149200 & 0.36449000 & 1.14130600 \\
\hline $\mathrm{C}$ & -8.44814600 & 0.38916400 & 0.82481200 \\
\hline 0 & -11.95060900 & -0.64637600 & 0.92214000 \\
\hline 0 & -10.98622800 & -2.52906600 & -0.70262300 \\
\hline $\mathrm{C}$ & 4.28698700 & 2.88668200 & -1.37141400 \\
\hline 0 & 3.66193300 & 3.75039800 & -0.77994800 \\
\hline 0 & 4.70409800 & 3.02721400 & -2.63819000 \\
\hline $\mathrm{H}$ & 0.58226000 & 2.02044200 & 1.40145100 \\
\hline $\mathrm{H}$ & 0.27840200 & 2.06530600 & -1.64309000 \\
\hline $\mathrm{H}$ & -1.67665600 & 2.96142300 & 0.52368800 \\
\hline $\mathrm{H}$ & -1.86417000 & 0.56588700 & -1.38257800 \\
\hline $\mathrm{H}$ & 0.27776200 & -0.25785000 & -0.63405200 \\
\hline $\mathrm{H}$ & -5.69195000 & 1.11664400 & 0.61182500 \\
\hline $\mathrm{H}$ & -6.17863400 & -1.41733200 & -1.05574500 \\
\hline $\mathrm{H}$ & -1.62410800 & 4.01369800 & -1.61328800 \\
\hline $\mathrm{H}$ & 1.45772000 & 3.84768100 & -0.77365400 \\
\hline $\mathrm{H}$ & 0.10176800 & -1.70944600 & 1.39183000 \\
\hline $\mathrm{H}$ & 0.57838200 & -0.32379600 & 2.41861800 \\
\hline $\mathrm{H}$ & 4.97015000 & 2.19912900 & 1.22058000 \\
\hline $\mathrm{H}$ & 3.43268500 & -3.87412800 & -1.03667900 \\
\hline $\mathrm{H}$ & 4.50866500 & -0.00882600 & 2.31690200 \\
\hline $\mathrm{H}$ & 4.13337500 & 0.77518800 & -1.37354800 \\
\hline $\mathrm{H}$ & 5.74087600 & 1.37787500 & -1.03099200 \\
\hline $\mathrm{H}$ & 8.47172200 & -2.54143600 & -0.16957600 \\
\hline $\mathrm{H}$ & 6.80225900 & -4.80427700 & -1.60910900 \\
\hline $\mathrm{H}$ & -8.32347900 & -2.34204500 & -1.21543600 \\
\hline $\mathrm{H}$ & -10.23726600 & 1.10939100 & 1.79870200 \\
\hline $\mathrm{H}$ & -7.82496900 & 1.16942600 & 1.24757500 \\
\hline $\mathrm{H}$ & -12.35538800 & -1.39911400 & 0.46092200 \\
\hline $\mathrm{H}$ & -10.55842600 & -3.15622100 & -1.30154400 \\
\hline $\mathrm{H}$ & 4.40906900 & 3.89993400 & -2.95369600 \\
\hline
\end{tabular}


Cartesian coordinates of the lowest-energy conformer of $4 \mathrm{C}(\mathbf{4 C}-1)$ at the B3LYP/6-31G(d,p) level in $\mathrm{MeOH}(\mathrm{PCM})$.

\begin{tabular}{|c|c|c|}
\hline-0.67443700 & 1.55389600 & -0.47563900 \\
\hline-0.08523600 & 2.36164800 & 0.68539100 \\
\hline 1.44587400 & 2.31800300 & 0.58600700 \\
\hline 1.92736500 & 0.87003100 & 0.48182000 \\
\hline 1.28590200 & 0.20990900 & -0.60475200 \\
\hline-0.12925500 & 0.11580000 & -0.41881000 \\
\hline 3.30583800 & 0.88342400 & 0.18567500 \\
\hline 4.05139900 & -0.19257600 & 0.61381200 \\
\hline 5.44828600 & -0.03116000 & 0.20808500 \\
\hline 3.57220000 & -1.11858700 & 1.24365800 \\
\hline 6.36352200 & -0.96943000 & 0.53749500 \\
\hline 7.78386600 & -0.98384600 & 0.22800700 \\
\hline 2.03536600 & 2.91469100 & 1.72569900 \\
\hline-0.44872200 & 3.73222100 & 5844000 \\
\hline-2.11569900 & 1.64841200 & -0.5 \\
\hline-0.67159200 & -0.79895600 & -1.51670400 \\
\hline-2.06975200 & -1.07975000 & -1.27907600 \\
\hline-2.34416300 & -2.05304800 & -0.37657500 \\
\hline-1.48514900 & -2.74959200 & 0.13154400 \\
\hline-3.80226600 & -2.20638200 & -0.12713800 \\
\hline-2.89972100 & 1.08999200 & 0.38232600 \\
\hline-2.50477800 & 0.47399600 & 1.35060200 \\
\hline-4.3720350 & 1.28212800 & 0.03512700 \\
\hline-4 . & -3 & \\
\hline-5.5 & -3 & \\
\hline-6.53789800 & -2.85550600 & 0.25753600 \\
\hline-6.12218400 & -1.64163200 & -0.26374100 \\
\hline-4.79453100 & -1.26272700 & -0.44606200 \\
\hline-7.03535600 & -0.69836100 & -0.71296300 \\
\hline-6.32755500 & 0.34518300 & -1.27142800 \\
\hline-4.82577700 & 80100 & -0.98112600 \\
\hline-4 . & 2 . & -0 . \\
\hline-6 & 1.2 & 2900 \\
\hline-7 . & -3 . & 100 \\
\hline-5.8 & -4 . & 5200 \\
\hline 8.41818 & 0.0 & -0 \\
\hline 9.77428600 & -0.02476800 & -0.77706700 \\
\hline 10.54369900 & -1.11610800 & -0.31140100 \\
\hline 9.92852000 & -2.13018900 & 0.41858900 \\
\hline 8.56257900 & -2.06274000 & 0.68353200 \\
\hline 11.87290100 & -1.17323900 & -0.57589900 \\
\hline 10. & 728200 & -1.48153700 \\
\hline-4 . & 3. & \\
\hline-3 & 3. & 00 \\
\hline-4.6 & 4.9 & 5800 \\
\hline-0.35131200 & 1.99997700 & -1.42035400 \\
\hline-0.38520000 & 1.90020500 & 1.63467600 \\
\hline 1.7462070 & 2.83860900 & -0.33683400 \\
\hline 1.7507410 & 0.32402200 & 1.41586400 \\
\hline-0 . & -0.33610400 & 5430600 \\
\hline 5. & 0.8 & -0 \\
\hline 5. & -1 & 900 \\
\hline 1.5 & 3. & 00 \\
\hline-1.36134800 & 38000 & 0.98611300 \\
\hline-0.1019640 & -1.72892100 & -1.53119000 \\
\hline-0.63414800 & -0.32017200 & -2.49619500 \\
\hline-4.91423400 & 1.12893500 & 0.97193500 \\
\hline-3.4322910 & -4.16685000 & 0.68091200 \\
\hline-4.266162 & 478000 & -1.91189800 \\
\hline-5 . & 35500 & 752300 \\
\hline & 2. & -1.4 \\
\hline-8.43250 & -2 . & 0.12465600 \\
\hline-6 . & 582200 & 1 \\
\hline & 0.8 & -0.88090900 \\
\hline 10.5299320 & -2.96182000 & 0.76971800 \\
\hline 8 & -2.85723600 & 1.25224700 \\
\hline 12.1098260 & -0.38529300 & -1.09202000 \\
\hline 10 & 1.62545400 & -1.78045700 \\
\hline & 5. & \\
\hline
\end{tabular}


Cartesian coordinates of the lowest-energy conformer of 4D (4D-1) at the B3LYP/6-31G(d,p) level in $\mathrm{MeOH}(\mathrm{PCM})$.

\begin{tabular}{|c|c|c|c|}
\hline 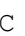 & -0.70864100 & 1.31851800 & -0.55411900 \\
\hline $\mathrm{C}$ & 0.02170000 & 2.59474100 & -0.13851900 \\
\hline $\mathrm{C}$ & 1.51489300 & 2.48909400 & -0.45596700 \\
\hline $\mathrm{C}$ & 2.06791600 & 1.21510000 & 0.17730100 \\
\hline D & 1.35315700 & 0.08523600 & -0.30886700 \\
\hline C & -0.02870100 & 0.07813000 & 0.04967400 \\
\hline 0 & 3.40387100 & 1.04949300 & -0.24082500 \\
\hline & 4.24684700 & 0.36481300 & 0.60601400 \\
\hline $\mathrm{C}$ & 5.58116200 & 0.26069600 & 0.01385200 \\
\hline 0 & 3.88761700 & -0.06699500 & 1.68708300 \\
\hline C & 6.57172100 & -0.36996100 & 0.68252600 \\
\hline $\mathrm{C}$ & 7.94898900 & -0.57192000 & 0.25967500 \\
\hline D & 2.21248900 & 3.60616600 & 0.06295300 \\
\hline & -0.46074900 & 3.74228600 & -0.80996900 \\
\hline & -2.04322900 & 1.42109400 & -0.01422500 \\
\hline$C$ & -0.55876100 & -1.24383100 & -0.49708200 \\
\hline 0 & -1.95155300 & -1.36486800 & -0.13479600 \\
\hline & -2.69421200 & -2.17863000 & -0.92062600 \\
\hline O & -2.21817900 & -2.83124400 & -1.83091800 \\
\hline C & -4.12637800 & -2.23729700 & -0.51768000 \\
\hline & -3.09931800 & 1.11550000 & -0.79153200 \\
\hline O & -3.04232100 & 0.81604600 & -1.96397600 \\
\hline C & -4.38359100 & 1.21292700 & 0.02433100 \\
\hline & -4.83352200 & -3.35234100 & -1.00651900 \\
\hline C & -6.15231400 & -3.60765800 & -0.64753700 \\
\hline & -6.81254100 & -2.74920700 & 0.25141000 \\
\hline & -6.09999900 & -1.65066100 & 0.70237300 \\
\hline C & -4.79155600 & -1.34016200 & 0.33719700 \\
\hline 0 & -6.64386900 & -0.76962300 & 1.62606900 \\
\hline & -5.67750800 & 0.15998300 & 1.94565800 \\
\hline & -4.46793100 & -0.01634000 & 1.01114900 \\
\hline & -4.44345900 & 2.58408200 & 0.75405400 \\
\hline & -5.85305500 & 0.96090800 & 2.82436500 \\
\hline 0 & -8.08410800 & -3.06571100 & 0.60651000 \\
\hline 0 & -6.78835700 & -4.70185300 & -1.14007600 \\
\hline$C$ & 8.82302500 & -1.25623400 & 1.13320700 \\
\hline$C$ & 10.14603700 & -1.47683600 & 0.78341200 \\
\hline$C$ & 10.63490800 & -1.01878700 & -0.45642900 \\
\hline & 9.77914100 & -0.34198300 & -1.32798300 \\
\hline C & 8.45283100 & -0.12044800 & -0.97542600 \\
\hline & 11.92905500 & -1.23250900 & -0.80448500 \\
\hline & 11.06974300 & -2.12659200 & 1.55767100 \\
\hline & -4.31499700 & 3.73186300 & -0.22554300 \\
\hline & -3.27095500 & 4.26928900 & -0.55792800 \\
\hline & -5.50093700 & 4.09910900 & -0.72982900 \\
\hline & -0.76326500 & 1.24194700 & -1.64255500 \\
\hline & -0.08457100 & 2.70808100 & 0.95242000 \\
\hline & 1.64192400 & 2.40812900 & -1.54646700 \\
\hline $\mathrm{H}$ & 2.02101700 & 1.25631300 & 1.27165700 \\
\hline & -0.14616100 & 0.10632700 & 1.14339200 \\
\hline & 5.71728300 & 0.70791800 & -0.96428200 \\
\hline $\mathrm{H}$ & 6.31239200 & -0.77787200 & 1.65824300 \\
\hline $\mathrm{H}$ & 1.68899400 & 4.38172600 & -0.19194600 \\
\hline H & -1.38365300 & 3.90617100 & -0.53782400 \\
\hline $\mathrm{H}$ & -0.00035800 & -2.07740500 & -0.06297500 \\
\hline $\mathrm{H}$ & -0.45570500 & -1.27935400 & -1.58276000 \\
\hline & -5.20912800 & 1.14111900 & -0.68764200 \\
\hline $\mathrm{H}$ & -4.32815800 & -4.04420100 & -1.66880900 \\
\hline $\mathrm{H}$ & -3.55871900 & -0.03906800 & 1.61741400 \\
\hline $\mathrm{H}$ & -3.62810700 & 2.65911100 & 1.47394600 \\
\hline 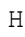 & -5.39137800 & 2.67982800 & 1.28130500 \\
\hline $\mathrm{H}$ & -8.43318200 & -2.41849200 & 1.23744000 \\
\hline & -7.68565000 & -4.73100700 & -0.77176000 \\
\hline $\mathrm{H}$ & 8.45604200 & -1.61477900 & 2.09156400 \\
\hline $\mathrm{H}$ & 10.17193000 & 0.00332900 & -2.27848100 \\
\hline & 7.80650800 & 0.40686500 & -1.66828800 \\
\hline & 12.35913100 & -1.71212700 & -0.07769700 \\
\hline & 10.67544600 & -2.40913300 & 2.39418700 \\
\hline & -5.34604200 & 4.81582800 & -1.37135500 \\
\hline
\end{tabular}

